\title{
PHYLOGENETIC RELATIONSHIPS AND TAXONOMIC REVISION OF THE BLENNIID FISH GENERA cirripectes AND Scartichthys
}

By

JEFFREY TAYLOR WILLIAMS

A DISSERTATINN FRFSENTED TO THE GRADIIATE SCHOOL OF THE UNIVERSITY OF FLORIDA IN PARTIAL FULFILLMENT OF THE REQUIREMENTS FOR THE DEGREF, OF DOCTOR OF PHILOSOPHY

UNIVERSITY OF FLORIDA 


\section{ACKNOWLEDGEMENTS}

During my work on this project, many people have assisted me in various ways. I thank all of them. In particular, I thank W. F. Smith-Vaniz, E. Bohlke, W. Saul, and staff (Academy of Natural Sciences of Philadelphia); P. C. Heemstra (J. L. B. Smith Institute of Ichthyology); T. Iwamoto, W. N. Eschmeyer, L. Dempster, and staff (California Academy of Sciences); R. Winterbottom and staff (Royal Ontario Museum); C. R. Gilbert, G. Burgess and J. B. Miller (F1orida State Museum); K. Harte1 (Museum of Comparative Zoology, Harvard); M. L. Bauchot and M. Desoutter (Muséum National d'Histoire Naturelle); A. G. Gerberich, K. A. Bruwelheide, L. P. Norrod, S. L. Jewett, J. R. Gomon, and Victor G. Springer (Smithsonian Institution), and the staff at all other museums that provided specimens, radiographs, and/or other technical assistance. I thank V. G. Springer, W. F. Smith-Vaniz, and H. Bath for their gracious hospitality while I was visiting museums. For providing color transparencies of specimens, I thank J. E. Randall, V. G. Springer, R. Winterbottom, and B. Carlson.

C. R. Gilbert and V. G. Springer generously gave their time to discuss my project. I gratefully acknowledge their help.

Financial support for this study was provided in part by the Department of Zoology and Florida State Museum, University of Florida; the California Academy of Sciences; and the National Science Foundation 
(Predoctoral Improvement Grant DEB 8207313). I thank my parents for their support throughout the study.

Finally, I thank my wife, Karen, for her support, editorial comments, and seemingly 1 imitless understanding. 
TABLE OF CONTENTS

PAGE

ACKNOWLEDGEMENTS................................. ii

ABSTRACT. ...................................... vi

inTRODUCTION.................................... 1

HISTORICAL TREATMENT OF THE BLENNIIDAE.................. 6

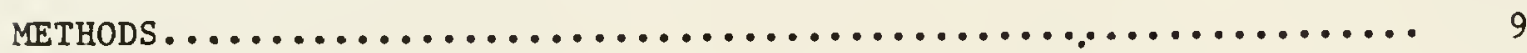

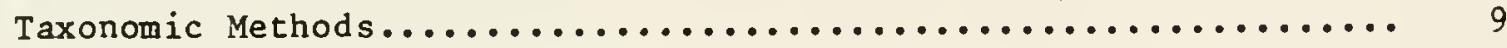

Methods of Phylogenetic Analysis....................... 10

PHYLOGENETIC ANALYSIS............................... 12

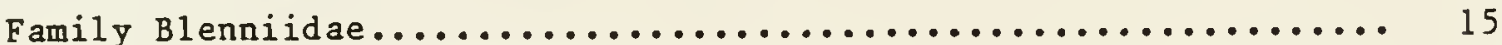

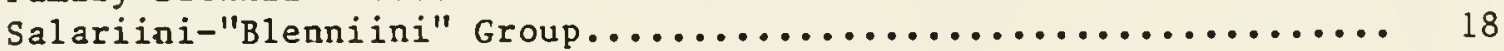

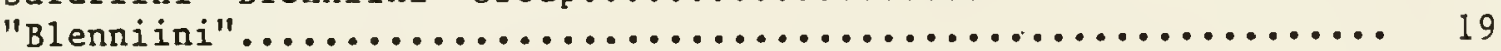

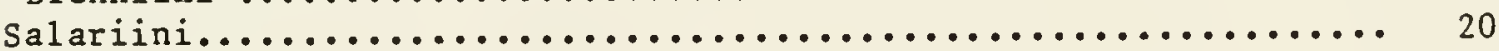

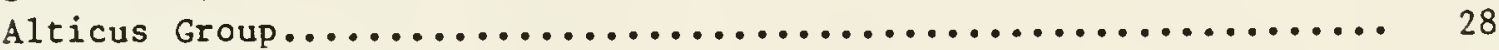

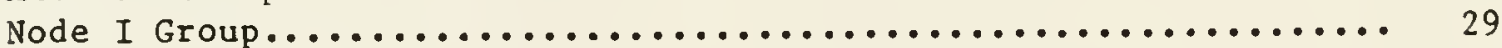

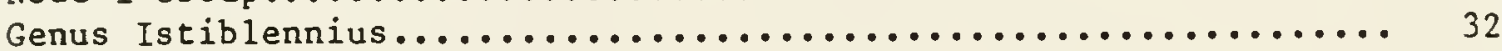

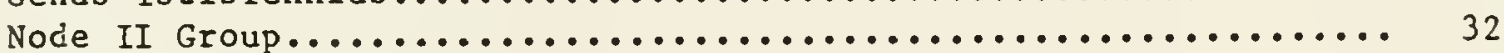

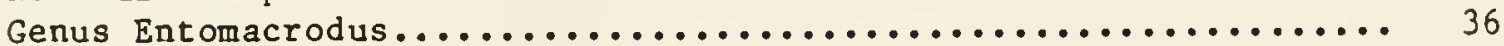

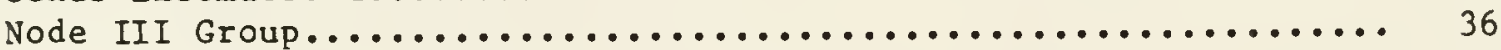

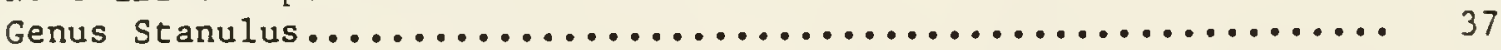

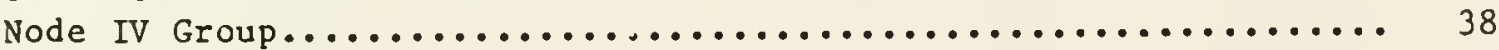

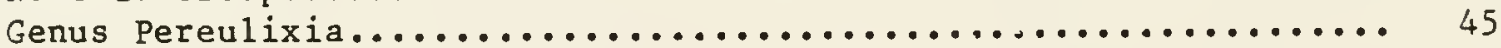

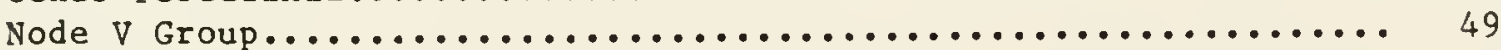

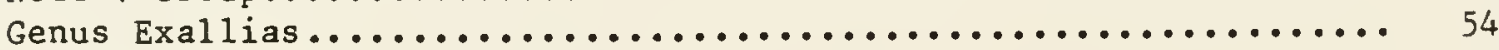

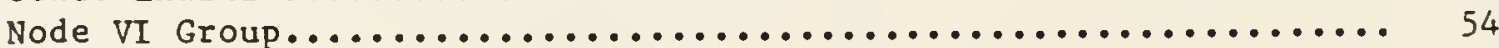

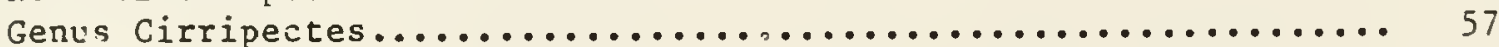

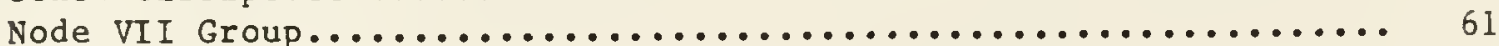

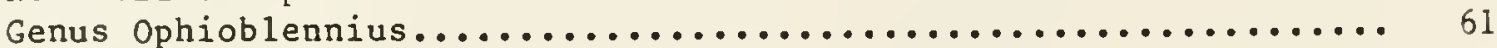

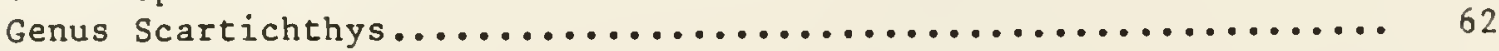

DISCUSSION OF PHYLOGENETIC ANALYSIS OF GENERA.............. 63

PHYLOGENETIC RELATIONSHIPS OF CIRRIPECTES SPECIES............ 58

Node VIII Group................................. 68

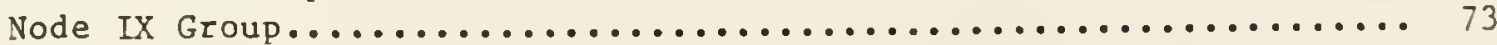

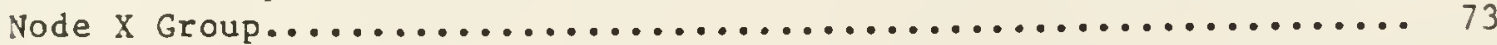


Node XI Group..................................... 76

Node XII Group..................................... 76

Node XIII Group...................................... 77

Node XIV Group.................................... 80

Node XV Group....................................... 80

Node XVI Group..................................... 81

Node XVII Group................................. 82

Cirripectes SWAINSON............................... 87

Key to the Species of Cirripectes.......................... 109

Cirripectes alboapicalis (ogilby)...................... 143

Cirripectes auritus Carlson............................ 149

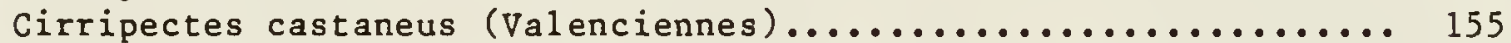

Cirripectes chelomatus Williams and Mauge...................... 165

Cirripectes filamentosus (Alleyne and Macleay)............... 170

Cirripectes fuscoguttatus Strasburg and schultz.............. 180

Cirripectes githerti new species.......................... 184

Cirripectes hutchinsi new speçies........................ 189

Cirripectes imitator Williams.......................... 195

Cirripectes jenningsi schultz............................ 199

Cirripectes kuwamurai Fukao............................ 203

Cirripectes obscurus (Borodin)........................... 205

Cirripectes perustus Smith............................. 209

Cirripectes polyzona (Bleeker)........................... 213

Cirripectes quagga (Fowler and Ba 1)..................... 219

Cirripectes randalli new species....................... 226

Cirripectes springeri new species....................... 230

Cirripectes stigmaticus strasburg and schultz.............. 234

Cirripectes vanderbilti (Fowler)........................ 241

Cirripectes variolosus (Valenciennes)................... 246

Scartichthys JORDAN AND EVERMANn....................... 254

Key to the Species of Scartichthys........................ 260

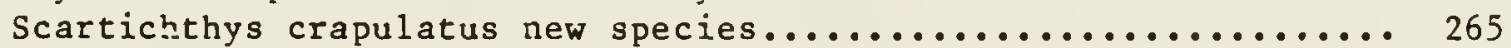

Scartichthys gigas (Steindachner)...................... 267

Scartichthys variolatus (Valenciennes).................. 272

Scartichthys viridis (Valenciennes) $\ldots \ldots \ldots \ldots \ldots \ldots \ldots \ldots \ldots \ldots . \ldots \ldots$

Exal lias JORDAN AND EVERMANN......................... 282

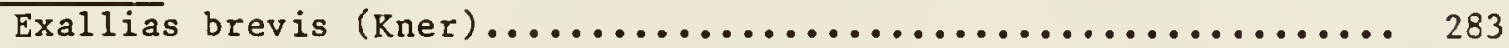

HISTORICAL ZOOGEOGRAPHY.......................... 291

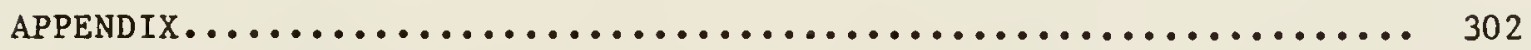

LITERATURE CITED................................ 307

BIOGRAPHICAL SKETCH............................ 314 
Abstract of Dissertation Presented to the Graduate School of the University of Florida in Partial Fulfillment of the

Requirements for the Degree of Doctor of Philosophy

\section{PHYLOGENETIC RELATIONSHIPS AND TAXONOMIC \\ REVISION OF THE BLENNIID FISH GENERA \\ Cirripectes AND Scartichthys}

By

Jeffrey Taylor Williams

May 1986

Chairman: Carter R. Gilbert

Major Department: Zoology

The blennid fish tribe Salariini comprises a group of marine shore fishes found circumglobally in tropical and subtropical seas. Members of the tribe are quite similar ecologically, with most being herbivores or deposit feeders and inhabiting rocky or coralline areas.

The salariin genera Cirripectes, Scartichthys, Exallias,

Ophioblennius, and Pereulixia form a monophyletic group closely related to the genera Stanulus, Entomacrodus, and Istiblennius. A phylogenetic analys is supports the hypothesis that each of the following genera is the sister genus to a combination of all preceding genera:

Scartichthys, Ophioblennius, Cirripectes, Exallias, Pereulixia,

Stanulus, Entomacrodus, and Istiblennius (including at least the $\underline{I}$. gibbifrons species complex). A hypothesis of the relationships among the species of Cirripectes is illustrated in a cladogram. 
The genera Cirripectes and Scartichthys are taxonomically revised. Cirripectes occurs throughout the Indo-Pacific region and comprises the

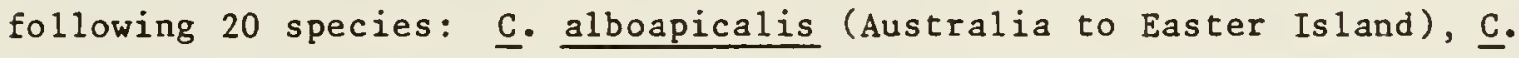

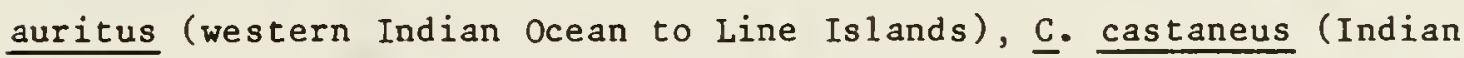
Ocean and Red Sea to western Pacific Ocean), C. chelomatus (Coral Sea to Tonga Islands), $\underline{\mathrm{C}}$. filamentosus (western Indian Ocean to western Pacific Ocean), $\underline{C}$. fuscoguttatus (Pacific Plate), $\underline{C}$. gilberti new

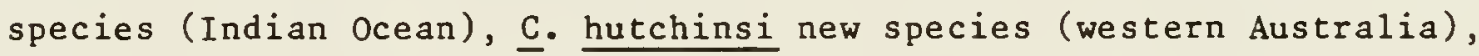
C. imitator (Taiwan to Japan and Ogasawara [Bonin] Islands), $\underline{\text {. }}$ jenningsi (Gilbert Islands to Tuamotu Archipelago), C. kuwamurai

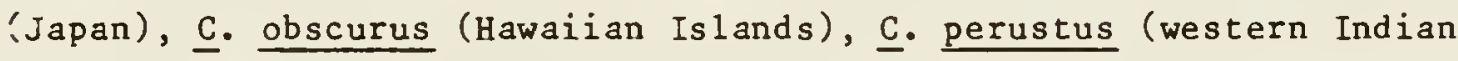

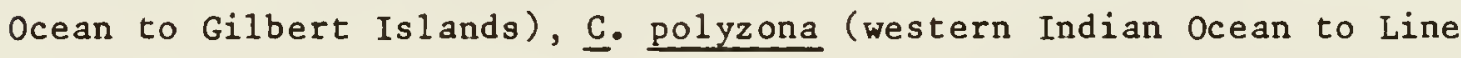

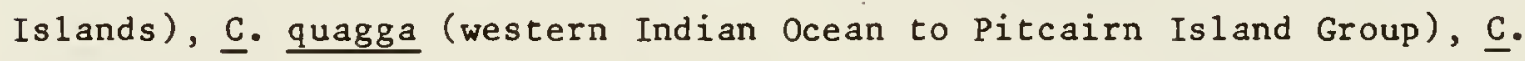

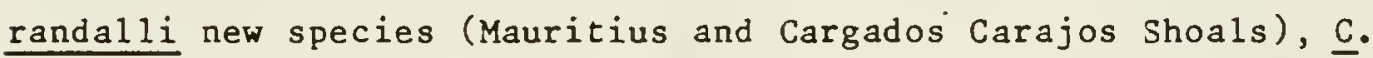

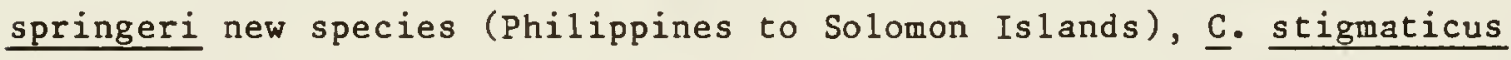

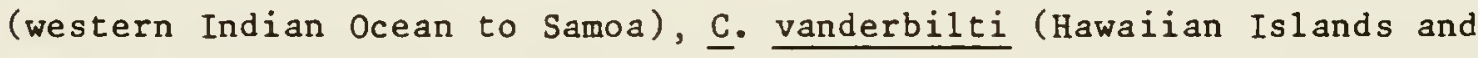
Johnston Island), and C. variolosus (Pacific Plate).

Scartichthys is restricted to the eastern Pacific Ocean and

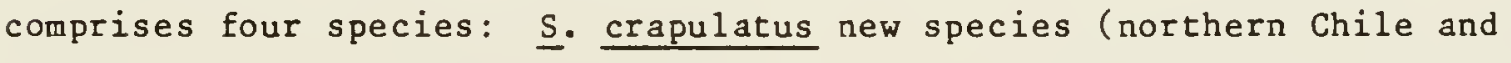

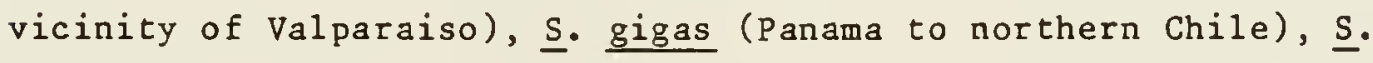
variolatus (San Felix, San Ambrosio, and Juan Fernandez Islands), and S. viridis (vicinity of Valparaiso to southern Peru).

Area cladograms derived from the phylogenetic analyses are analyzed. Eleven geographic patterns indicated by the cladogram are discussed. 


\section{INTRODUCTION}

The blenniid fish tribe Salariini comprises a group of marine shore fishes found circumglobally in tropical and subtropical seas. Members of the tribe are quite similar ecologically; most are herbivores or deposit feeders and inhabit rocky or coralline areas.

The objectives of the present study are to 1) test the hypotheses that the genera Cirripectes, Scartichthys, Ophioblennius, Pereulixia and Exallias comprise a monophyletic group and are each monophyletic; 2) if the first hypotheses are confirmed, form phylogenetic hypotheses of interrelationships of the species of Cirripectes; 3) taxonomically revise Cirripectes and Scartichthys, and provide complete species descriptions of and distribution maps for each of the included species; and 4) analyze the distribution patterns of these genera and their included species.

Smith-Vaniz and Springer (1971) gave a synopsis of the Salariini to provide a foundation for future work on the tribe. They pointed out several genera that needed additional study and provided a preliminary hypothesis of the relationships of the 24 genera they recognized in the tribe. Their method was not cladistic, but they did establish the monophyletic nature of the tribe Salariini. Although they did not cladistically analyze the members of the tribe, they provided a dendrogram (Smith-Vaniz and Springer, 1971: fig. 51) of the genera 
showing their interrelationships. This dendrogram suggests that the genera Cirripectes, Scartichthys, Exallias, Pereulixia, and Ophioblennius are more closely related to each other than to other members of the tribe.

The genus Cirripectes Swainson is widespread throughout the Indo-Pacific. It is a common inhabitant of coral and rocky reefs and is usually common in heavy surge areas. Specimens of Cirripectes are common in museum collections, but complex nomenclatural problems and the lack of adequate keys to the species have resulted in numerous misidentifications. Smith-Vaniz and Springer (1971) 1isted 24 nominal species of Cirripectes, and four additional species have since been described (Table 1). There has been no comprehensive revision of the genus and all published keys to species have been of a regional nature (Chapman, 1951, for the Indo-Australian Archipelago; Fukao, 1984, for Japan; Schultz and Chapman, 1960, for the Marshall and Mariana Islands; Smith, 1959, for the western Indian Ocean). As a result, numerous populations from different geographic areas have been described as new species, even though these populations are actually members of the same widespread species. There is also sexual polychromatism in some species and, in many instances, each color morph has been named as a distinct species. Like most genera of marine shore fishes, there has been no phylogenetic analysis of the interrelationsins of the Cirripectes speciés. 
Table 1. List of nominal species of Cirripectes with correct identifications. Arrangement is alphabetical by species name.

Species, Author, Publication Date

Salarias alboapicalis Ogilby, 1899

Cirripectes auritus Carlson, 1981

Blennius canescens Garman, 1903

Ophioblennius capillus Reid, 1943

Salarias castaneus Valenciennes in Cuv. and Val., 1836

Cirripectes chelomatus Williams and Mauge, 1983

Ophioblennius clarki Reid, 1943

Salarias cruentipinnis Day, 1888

Cirripectes cruentus J. L. B. Smith, 1959

Salarias filamentosus Alleyne and Macleay, 1877

Cirripectes fuscoguttatus Strasburg and Schultz, 1953

Cirripectus gibbifrons J. L. B. Smith, 1947

Cirripectes imitator Williams, 1985

Cirripectes indrambaryae H. M. Smith, 1934

Cirripectes jenningsi Schultz, 1943

Cirripectes kuwamurai Fukao, 1985

Cirripectes 1 ineopunctatus Strasburg, 1956

Salarias nigripes Seale, 1901

Exallias $\overline{\text { obscurus }}$ Borodin, 1927

Cirripectes perustus J. L. B. Smith, 1959

Salarias (Cirripectes) polyzona Bleeker, 1868

Rupiscartes quagga Fowler and Ball, 1924

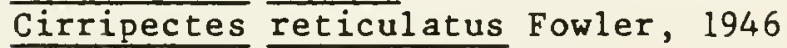

Salarias sebae Valenciennes in Cuv. and

$$
\text { Val., } \frac{1836}{186}
$$

Cirripectes stigmaticus Strasburg and Shultz, 1953 Ophioblennius vanderbilti Fowler, 1938

Salarias variolosus Valenciennes in Cuv. and Val., 1836

Cirripectus variolosus patuki De Buen, 1963
Correct Identification

alboapicalis

auritus

polyzona

vanderbilti

castaneus

chelomatus

variolosus

filamentosus

stigmaticus

filamentosus

fuscoguttatus

castaneus

imitator

filamentosus

jenningsi

kuwamurai

quagga

variolosus

obscurus

perustus

polyzona

quagga

castaneus

castaneus

stigmaticus

vanderbilti

variolosus

alboapicalis 
Cohen (1956) briefly reviewed the eastern Pacific genus Scartichthys Jordan and Evermann. He examined specimens of several nominal species, provided a partial synonymy tor the genus, and

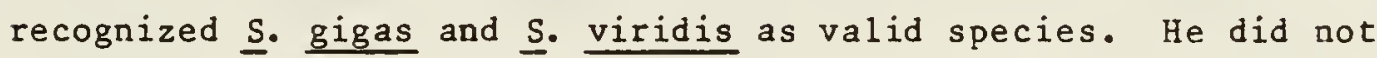
attempt to treat all of the nominal species, thus leaving the status of many species in doubt. Smith-Vaniz and Springer (1971) listed thirteen nominal species (Table 2) and estimated that there were only two valid species of Scartichthys.

Ophioblennius Gill (reviewed by Springer, 1962), Pereulixia Smith, and Exallias Jordan and Evermann, and their individual species, are relatively well-defined, but their interrelationships within the Salariini have not been formally hypothesized.

Concerning generic relationships, Smith-Vaniz and Springer (1971:20) suggested that Scartichthys might be congeneric with Cirripectes, but deferred combining them until more detailed studies were made. Their statement (p. 20) about the relationship between these two genera is inconsistent with statements nade elsewhere in their paper. They suggested (p. 24) that Exallias appeared to be most closely related to Cirripectes and ( $p .36$ ) that Ophioblennius appeared to be most closely related to Scartichthys. Later in the paper (their Figure 51), they suggested that Cirripectes and Scartichthys are sister groups, which are in turn the sister group of Exallias, and that these three genera together form the sister group of Pereulixia. Ophioblennius is then shown as the sister group of all of these genera. This confusion concerning the relationships among these genera illustrates the need for a complete and thorough analysis of their interrelationships. 
Table 2. List of nominal species of Scartichthys with correct identifications. Arrangement is alphabetical by species name.

Species, Author, Publication Date

Salarias concolor Philippi, 1896

Salarias cuvieri Gunther, 1861

Salarias eques Steindachner, 1898

Ophioblennius fernandezensis Clark, 1938

Salarias gigas Steindachner, 1876

Ophioblennius mazorkae Hildebrand, 1946

Salarias modestus Philippi, 1896

Salarias petersoni Fowler, 1940

Salarias rubropunctatus Valenciennes in Cuv. and Val., 1836

Blennophis semifasciatus Kner and Steindachner, 1866

Salarias variolatus Valenciennes in Cuv. and Val., 1836

Salarias viridis Valenciennes in Cuv. and Val., 1836 Ophioblennius xiphiodon Clark, 1938
Correct Identification

viridis

viridis

gigas

variolatus

gigas

gigas

viridis

viridis

variolatus

viridis

variolatus

viridis

gigas 


\section{HISTORICAL TREATMENT OF THE BLENNIIDAE}

Springer (1968b) reviewed the literature dealing with classification of the Blenniidae. He cited numerous studies of higher level (family or above) relationships, but noted that Norman (1943) provided the only comprehensive intrafamilial classification. Norman (1943) recognized three blenniid subfamilies: Ophioblenniinae, Blenniinae, and Salariinae. Springer (1968b) split Norman's Blenuiinae into the subfamily Blenniinae, comprised of the tribes Salariini. Blenniini, and Omobranchini, and the subfamily Nemophidinae and noted that Norman's Ophioblenniinae was based on larval stage individuals belonging to several different genera, all but one of which (Somersia, Labrisomidae) he placed in the tribe Salariini. Springer and Smith-Vaniz (1972) chose not to recognize blenniid subfamilies, preferring instead to recognize five tribes in the family Blenniidae (Blenniini, Salariini, Omobranchini, Nemophini, and Phenablenniini). Springer (1968b) proposed that Blennius was most closely related to the Omobranchini and Nemophini Iine. Smith-Vaniz (1976), noting that the Blenniini of Springer and Smith-Vaniz (1972) was polyphyletic, removed Blennius (which would be the true Blenniini) from the Blenaiini, and hypothesized a sister group relationship between Blennius and a group comprised of the tribes Orobranctini, Phenablenniini, and Nemophini. 
Smith-Vaniz (1976) did not propose a hypothesis for the relationship of his monophyletic group to the remaining members of the Blenniidae, leaving the Salariini, "Blenniini," and Blennius in an unresolved trichotomy (his Figure 88 ).

In his revision of the tribe Blenniini, Bath (1977) commented on the sutured dentaries of Blennius but retained it in the tribe Blenniini. Bath stated that the tribe was defined by a group of unspecialized characters, thus leaving open the possibility that some of the genera would ultimately be removed.

Springer (1968b) stated that the Salariini were either offshoots of the Blenniini or shared a common ancestor with them. Smith-Vaniz and Springer (1971) and Springer and Smith-Vaniz (1972) supported a close relationship between the tribes Salariini and Blenniini, but proposed no distinctive characters to support this hypothesized relationship.

Smith-Vaniz and Springer (1971) provided data supporting the monophyletic nature of the tribe Salariini and discussed in detail the nature of the dentition and jaws. Although the lateral portions of the premaxillary capsule are enclosed in certain genera, they found that at least the mesial portion of each premaxilla was an open capsule (i.e. no anteroventral wall of bone) in all genera of the Salariini. Smith-Vaniz and Springer (1971) diagnosed all genera they recognized in the Salarini and presented their partially intuitive concept of the 
relationships among the zenera in a dendrogram (Smith-Vaniz and Springer, 1971:Figure 51). 
METHODS

\section{Taxonomic Methods}

Counts and measurements follow Smith-Vaniz and Springer (1971) and Williams (1985). Specimens used for osteological examination were prepared by clearing and counter-staining with Alizarin-red $S$ and sician blue following the method of Dingerkus and Uhler (1977). Counts nf vertical fin rays and characters associated with the vertebral column (i.e. pleural ribs, epipleural ribs, etc.) were taken from radiographs. When the last anal-fin ray was split through base, it was counted as one.

Species accounts are arranged alphabetically by species. Only primary synonyms are 1 isted. In the material examined section for each species, the presence of a standard length measurement associated with a catalog number indicates that counts and measurements were taken from that specimen(s). All other material listed was examined to confirm identification and for use in establishing geographic ranges.

Counts of dentary and premaxillary incisors are approximate due to the difficulty of counting these tiny teeth.

Institutional abbreviations used in iistings of material examined are from Leviton et al. (1985). Other abbreviations used are SL-standard length, HL-head length, LL--lateral line, AN--anterior 
nostril; EIP--extra interorbital sensory pore position; IFO-infraorbital sensory pore series; MD-mandibular sensory pore series; MSP--mid-snout pores; PBN-pore positions behind nuchal flap; PN-posterior nostril; POP-preopercular sensory pore series; So-supraorbital sensory pore series; ST-supratemporal sensory pore series.

\section{Methods of Phylogenetic Analysis}

Procedures used to determine relationships between taxa follow the general principles of Hennig (1966), as discussed by Vari (1978) and Parenti (1981). Only monophyletic groups (i.e. a group that includes all descendants and only descendants of a hypothetical common ancestor) are recognized as valid taxonomic units. Each monophyletic group is defined by shared derived characters (synapomorphies). Polarity of each character (i.e. the character state toward which the character is assumed to have evolved in the taxa being analyzed) is determined by outgroup analysis (Maddison et al., 1984). The principle of parsimony is used to resolve cases where derived (apomorphic) characters support conflicting hypotheses of relationship (i.e. homoplasiously distributed characters). Although evolution may not proceed in accordance with the principle of parsimony, this approach minimizes the number of assumptions that must be made to explain character transformations. 
The result of a phylogenetic analysis is a hierarchical arrangement of increasingly inclusive monophyletic groups. This arrangement, usually illustrated as a branching diagram (cladogram), proviaes a hypothesis of the phylogenetic relationships of the taxa analyzed. The phylogenetic method is the preferred technique for this study as it provides a clear hypothesis of relationships that can be tested further by examining other characters. 
PHYLOGENETIC ANALYSIS

In this section, I review the synapomorphies of the Blenniidae and other taxonomic groupings at decreasing levels of universality that lead to Cirripectes and its allies. Although much work remains to be done before the relationships of blenniid genera and species are fully resolved, this discussion will provide data necessary to support my use of certain taxa as outgroups for determining character polarities. Characters used to define groupings at higher taxonomic levels (i.e. family, tribe) are based on a general survey of selected species within each group. The major emphasis of my study is on Cirripectes and its allies in the Salariini. The relationships among the genera closely related to Cirripectes are depicted in the cladogram of Figure 1. Groupings of genera on this cladogram will be referred to in the text by the node number. Generic groupings outside the Cirripectes assemblage of Figure 1 are not shown on a cladogram because the relationships proposed herein for these taxa are tentative and require additional study.

The goal of this study is to provide a working phylogenetic hypothesis of blenniid relationships and should be considered a first step in the dynamic process of phylogenetic analysis. 


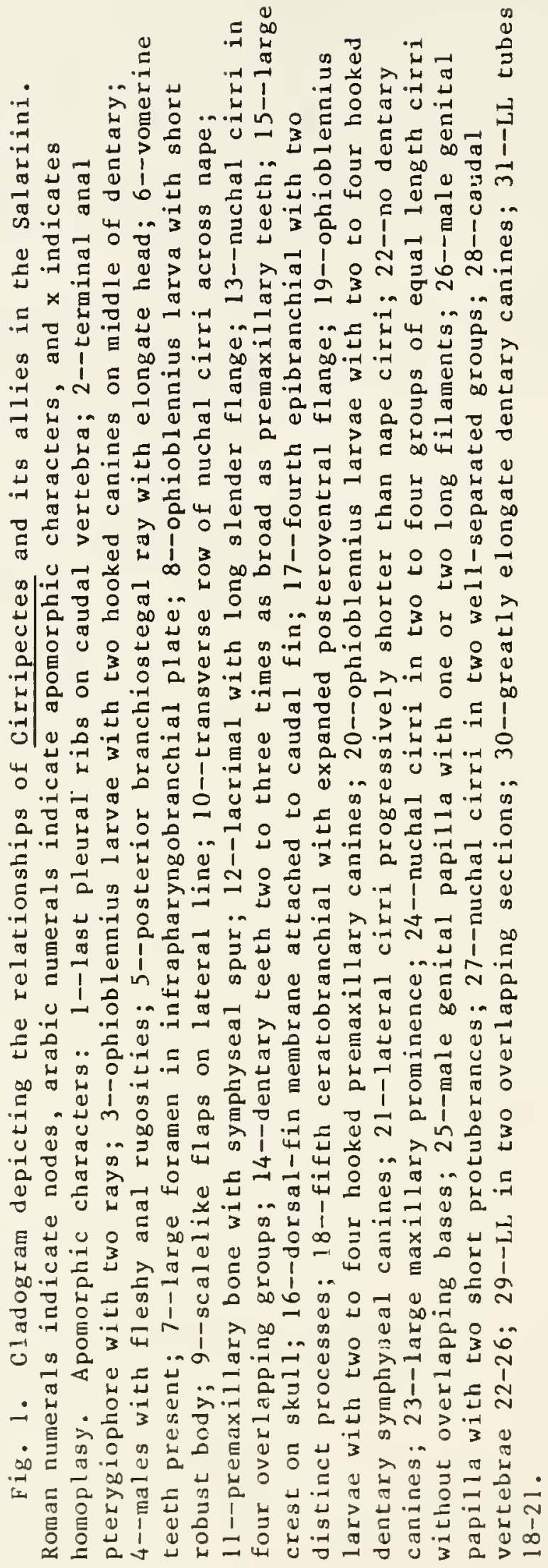




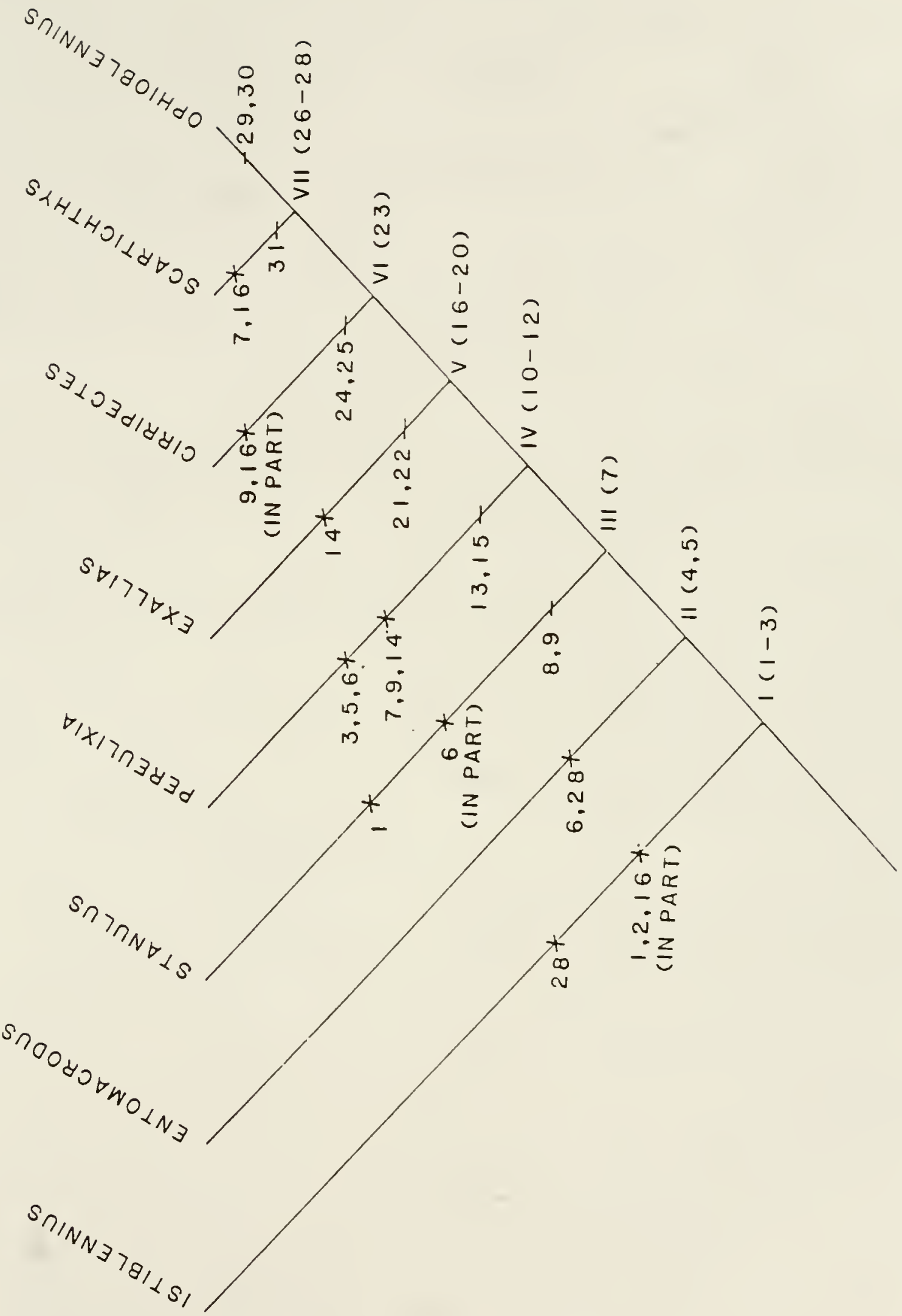


Family Blenniidae

Springer (1968b) proviled an extensive osteological description of the family Blenniidae, based primarily on Entomacrodus nigricans: He also discussed three characters that distinguish the Blenniidae from

all other blennioids: (1) coracoid reduced and fused to cleithrum; (2) distinctive relationship of the interopercle with the epihyal (=posterior ceratohyal) and interhyal; and (3) distinctive premaxillary and dentary dentition of adults.

In addition to these three characters, monophyly of the Blennidae is supported by two other shared derived characters. The first is a distinctive relationship of the urohyal with the dorsal and ventral hypohyals, a condition not found in any other blennioids. The blenniid urohyal has two lateral projections (one dorsal and one ventral) on each side (Figure 2), each with a strong ligamentous attachment to its respective hypohyal(s). There is variation in the positioning of the lateral projections among the bitaniid tribes, but all members have this distinctive urohyal-hypohyal association.

The other derived character shared by the Blenniidae is the presence of fleshy rugosities on the anal-fin spines of adult males. As anal-fin spine rugosities are not found in other blennioid fishes (sensu George and Springer, 1981), or other Parciform fishes, the 
Figure 2. Urohyal bone. (Anterior toward left; $A$ and C--left lateral views; $B$ and $D$--dorsal views.) $A$ and $B-$ no developed lateral processes (Acanthemblemaria aspera). C and D-well-developed lateral processes (Chasmodes saburrae). 
A

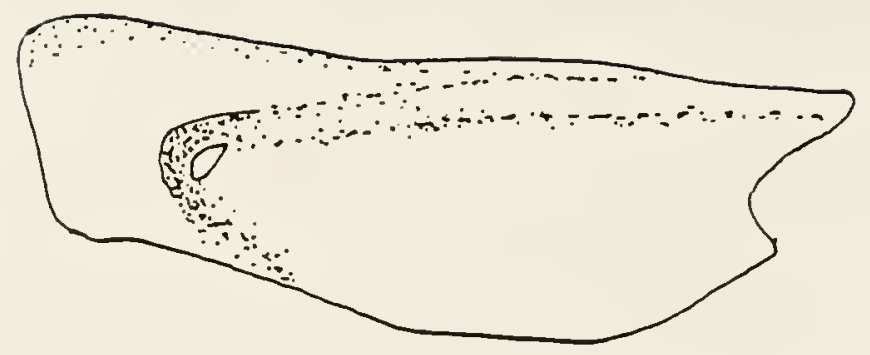

B
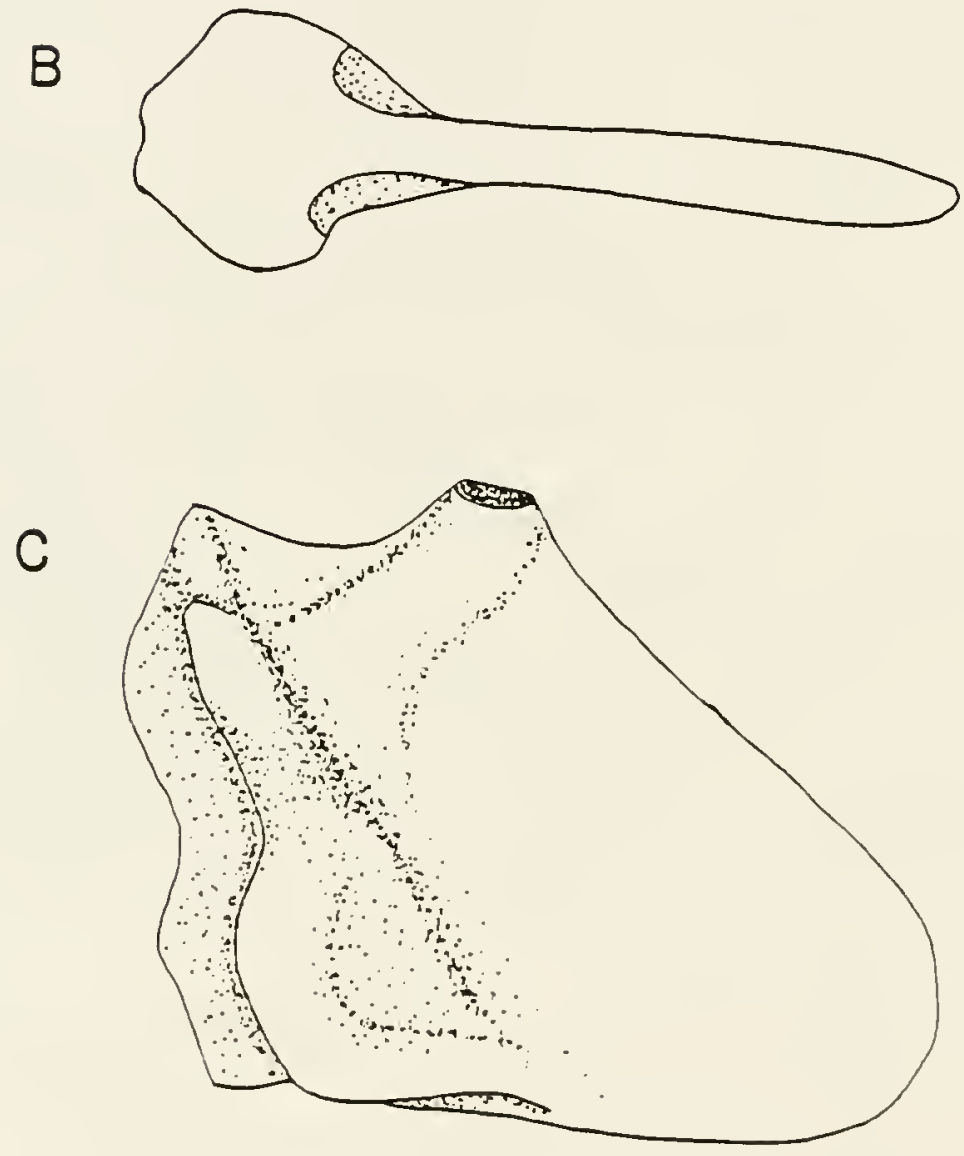

D

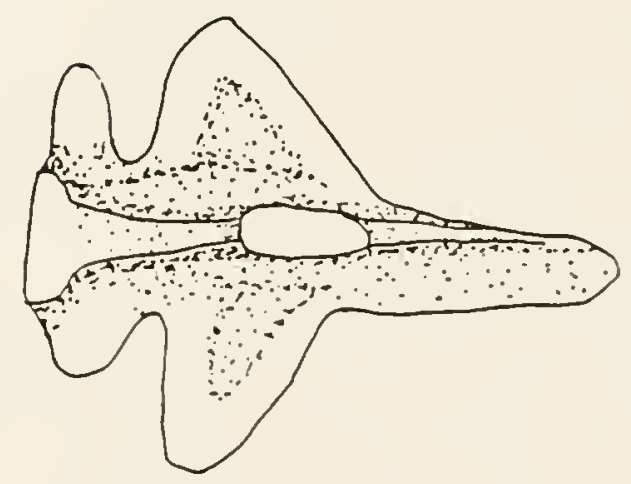


Presence of these fleshy rugosities is the apomorphic condition. The Omobranchini-Phenablenniini group of Smith-Vaniz (1976) and certain genera of the Salariini are the only Blenniidae not having anal-fin spine rugosities. The most parsimonious explanation (based on Smith-Vaniz's [1976] cladogram as modified in my discussion of the Salariini-"Blenniini" group) of this character's distribution among blenniid taxa is that the absence of anal-fin spine rugosities represent two independent reversals (losses), one in the Omobranchini-Phenablenniini group and one in the ancestral line giving rise to the Salariini, with a secondary derivation of the rugosities in one lineage within the Salariini.

\section{Salariini-"Blenniini" Group}

Springer (1968b) and Smith-Vaniz and Springer (1971) proposed a close relationship between the Salariini and Blenniini, but did not provide a haracter to support this relationship. I follow Smith-Vaniz (1976) in separating Blennius, which is now the only member of the true Blenniini, from the remaining genera of the tribe, which $I$ will refer to as the "Blenniini." A thorough study of the "Blenniini" is needed to determine whether it is monophyletic.

The hypothesized sister-group relationship between the "Blenniini" and Salariini is supported by two synapomorphies: (1) premaxillary ascending process only weakly attached to main body of bone, and (2) 2-0-2 pectoral radial formula. The ascending process of the 
premaxillary bone has an ossified connection to the main body of the bone only at the anteriormost and posteriormost edges, while the middle part is loosely attached by connective tissue. Springer (1968b) stated that the blenniid ascending process was weakly joined (ossified connection) at two points, anteriorly and posteriorly, to the ventral portion of the premaxilla. Although this is true of the Salariini-"Blenniini" line, other blenniids and blennioids have the entire base of the ascending process weakly or strongly connected by bone to the main body of the premaxilla. In addition, the ascending process is an independent ossification in Pereulixia and Scartichthys, where the anterior and posterior connections to the main body of the premaxilla have been replaced by connective tissue. Alticus has an intermediate condition in that a bony anterior connection has been lost, but the posterior connection has been retained.

Springer (1968b) proposed that the most primitive pectoral radial formula for blenniids is $2-1-1$. Therefore, the $2-0-2$ formula is considered to be a derived condition. Some members of both tribes have the 2-1-1 formula, which I interpret as a secondary reversion to the 2-1-1 condition.

\section{"Blenniini"}

As discussed previously, the "Blenniini" referred to here comprises those genera included in the tribe by Bath (1977), but excluding Blennius. 
Monophyly of the "Blenniini" is supported by two synapomorphies. The first character is the first basibranchial shaped like a broad, shallow U, curving downward between the posterior end of the basihyal and the anteroventral edge of the second basibranchial (Figure 3C). I have not found a similarly shaped basibranchial in any other blennioid examined and consider this condition as derived compared with the straight basibranchial of most other blennid genera or a basibranchial with the anterior end slightly upturned (Istiblennius, Antennablennius, Atrosalarias, Ecsenius, Entomacrodus, and Litobranchus).

The second character wisting the "Blenniini" is their distinctively shaped fourth epibranchial bones (Figure 4B). The "blenniin" fourth epibranchial is a relatively smooth bone with a flattened longitudinal bony flange projecting dorsally from the lateral half of the shaft of the bone. All other blennioids examined have a fourth epibranchial bone with one or two dorsally directed bony projections situated dorsally on the shaft slightly before the articulation of the epibranchial shaft with the infrapharyngobranchial plate. This type of projection is lacking in the "Blenniini."

These characters have been examined in only a small percentage of "blenniin" species. Thus, this hypothesis of monophyly for the tribe needs to be further tested.

\section{Salariini}

Two characters support monophyly of the Salariini:

premaxillary bone an open capsule and (2) distinctively shaped 
Figure 3. Left lateral view of basibranchial bone. (Anterior toward left.) A-plesiomorphic rod-shaped structure (Cirripectes filamentosus). B--slightly upturned anterior end (Atrosalarias fuscus). C-derived shallow-U shape of "Blenniini" (Chasmodes saburrae). 
A

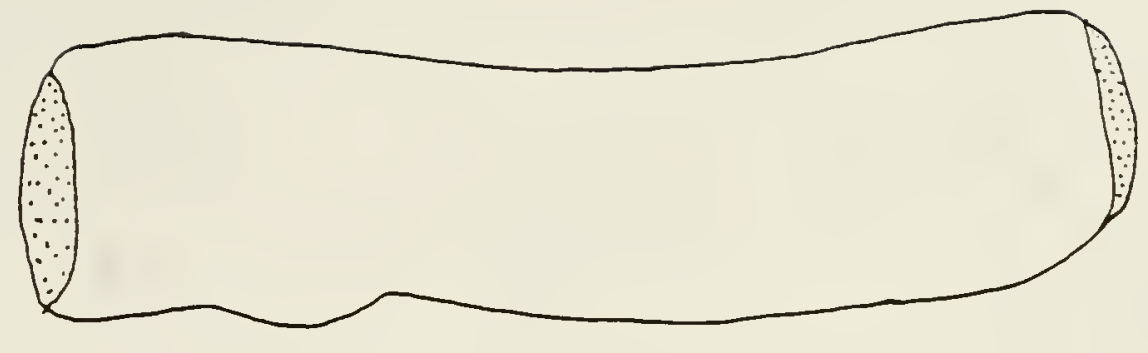

B

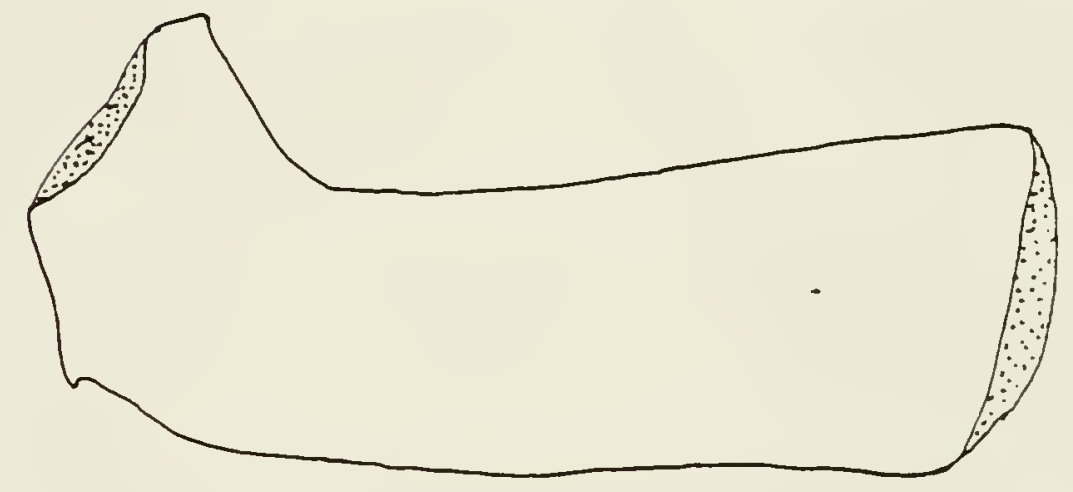

C

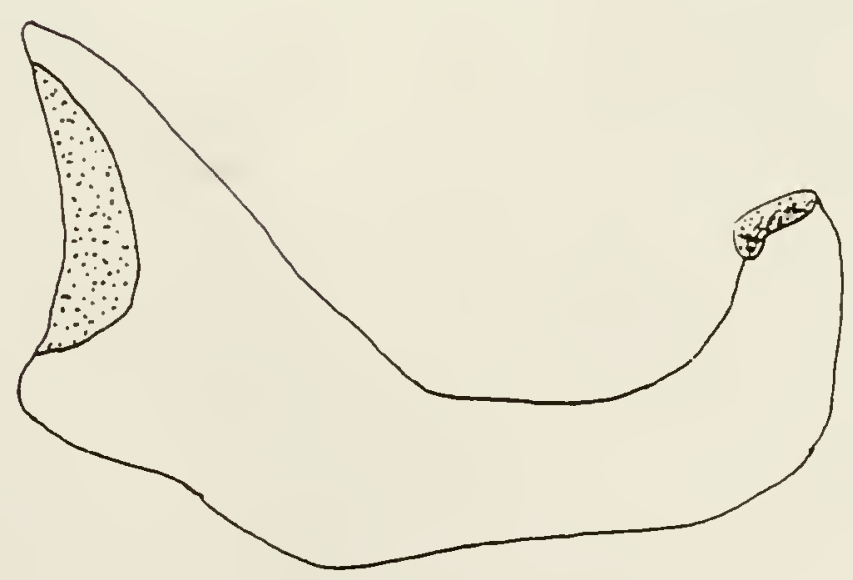


Figure 4. Fourth epibranchial bone. Left dorsolateral view. (Anterior toward left.) A-single, broad, mid-shaft process (Atrosalarias fuscus). B-no mid-shaft process (Chasmodes saburrae). C-two mid-shaft processes positioned at mid-shaft (Cirripectes filamentosus). Abbreviation: PR--mid-shaft process. 

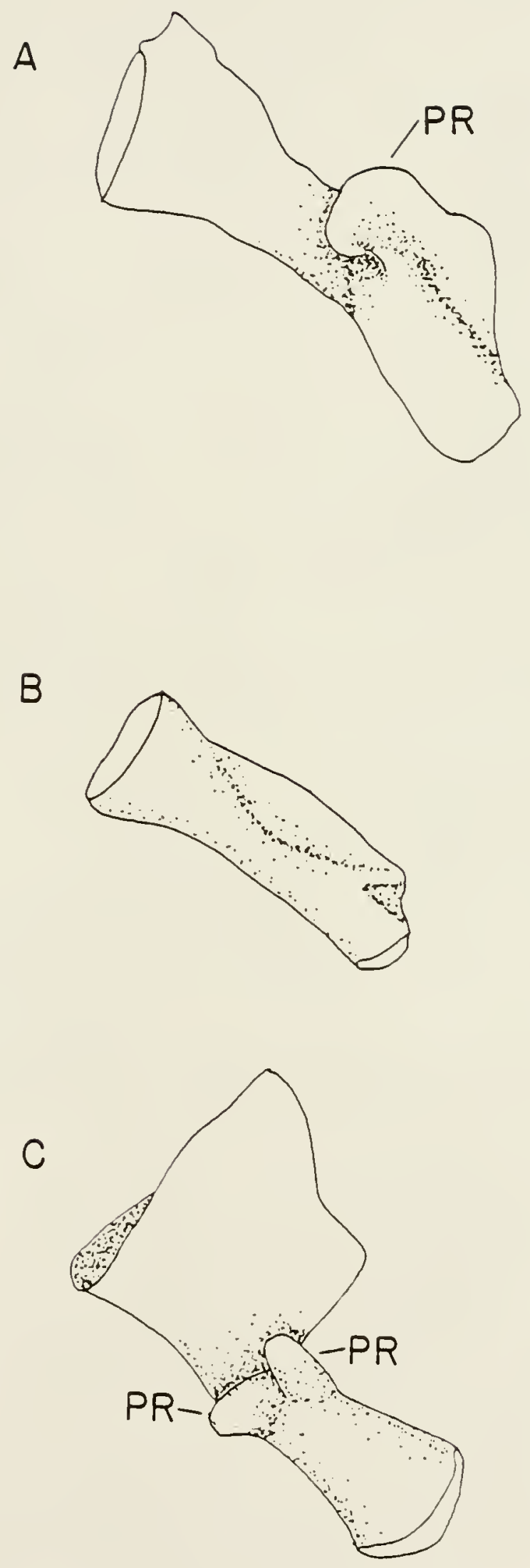
infrapharyngobranchial plate (Figure 5F-K). The first character was discussed in detail by Smith-Vaniz and Springer (1971). The premaxillary bone of the Salariini has a band of connective tissue in place of the anteroventral bony wall present in all other blennioid fishes.

The infrapharyngobranchial plate of the Salariini has a broad, flattened bony flange projecting medially from the tooth-bearing portion of the bone. This flange extends anteriorly to articulate with the medial tip of the second epibranchial. Further elaborations of the infrapharyngobranchial flange occurring in Cirripectes and its allies are discussed in the account for the node III group. In the Tripterygiidae, "Blenniini" (the hypothesized sister group of the Salariini), and all other groups in the family Blenniidae, the infrapharyngobranchial plate is composed primarily of the tooth-bearing portion with a narrow medial flange of bone that has an anterior rod-shaped protuberance articulating with the second epibranchial. The families Dactyloscopidae, Chaenopsidae, Clinidae, and Labrisomidae also have an infrapharyngobranchial plate with a large bony flange projecting anteromedially from the tooth-bearing portion, but the lateral edge of the flange is developed as a rod-shaped cartilaginous swelling. The expanded bony flange appears to have developed independently in the two groups and, thus, is considered a synapomorphy of the Salariini.

I recognize two major groups within the Salariini, which I refer to as the Alticus group and the Rhabdoblennius group. 
Figure 5. Dorsolateral views of left infrapharyngobranchial plates. (Anterior toward left.) A-Enneanectes altivelis.

$B$-Acanthemblemaria aspera. C-Chasmodes saburrae. D-Omobranchus punctatus. E-Meiacanthus gramistes. F-Atrosalarias fuscus. G-Stanulus seychellensis. $\mathrm{H}$-Ophioblennius atlanticus.

I-Cirripectes perustus. J-Cirripectes polyzona. K-Cirripectes quagga. Dashed line indicates anteriormost limit of tooth-bearing portion of bone. Abbreviations: F-medial flange; RC-rod-shaped cartillage. 
A

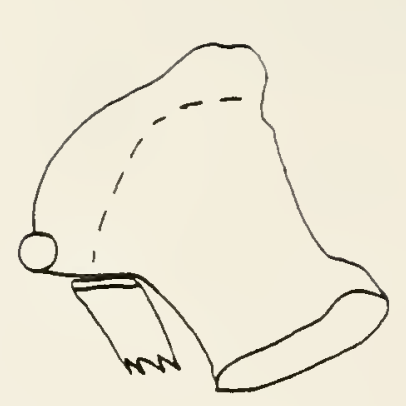

C

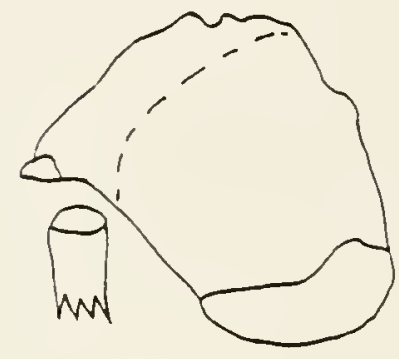

E

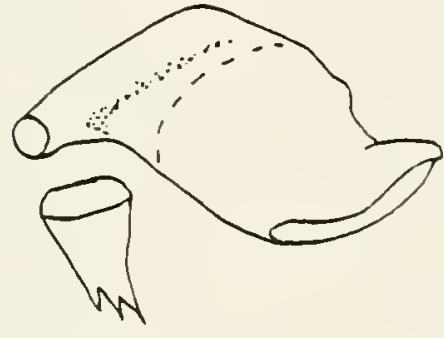

G
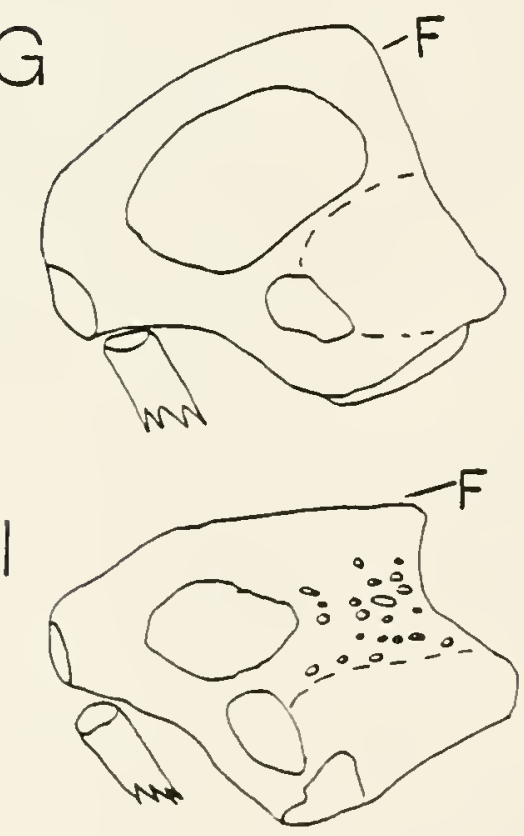

B

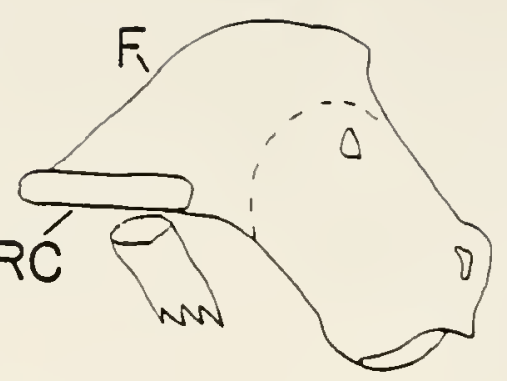

D

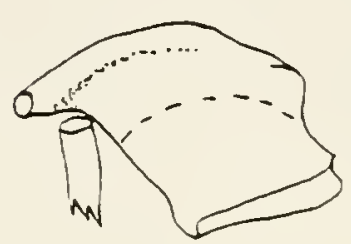

F
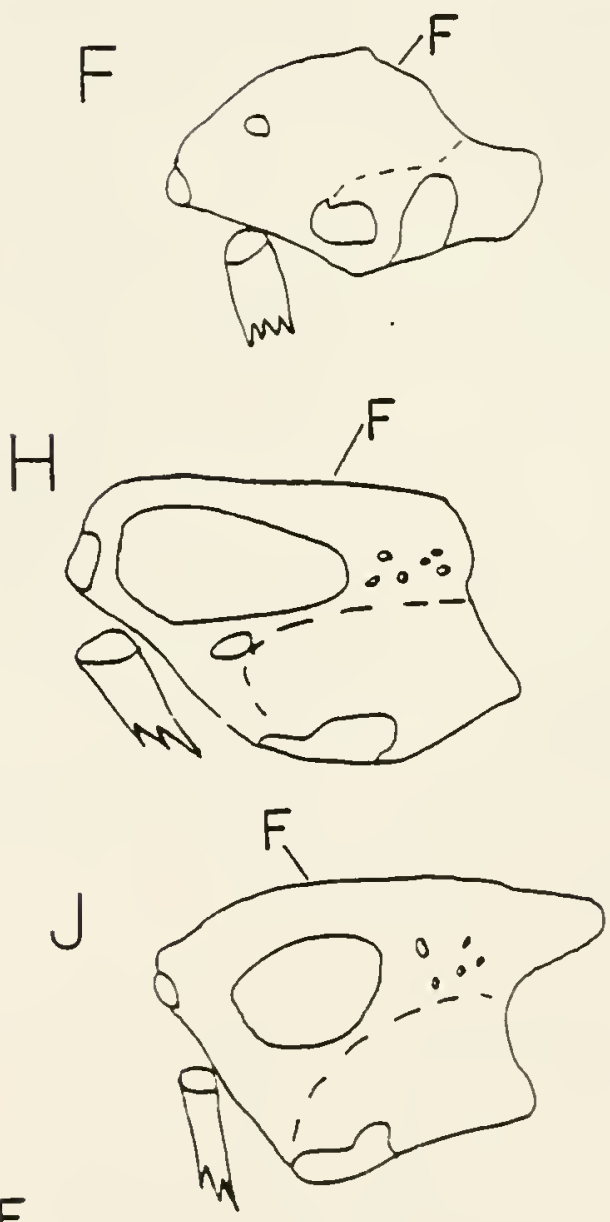

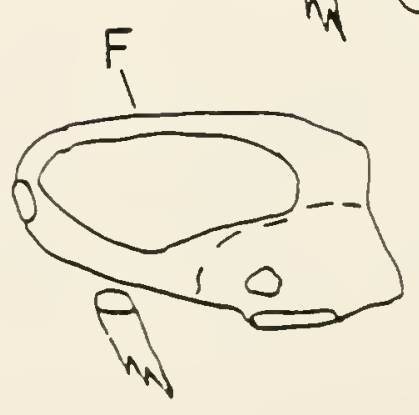




\section{Alticus Group}

The Alticus group comprises the genera Ecsenius, Andamia, Alticus, Dodekablennos, Praealticus, Istiblennius, Ophioblennius, Pereulixia, Exallias, Cirripectes, Scartichthys, Stanulus, Entomacrodus, Nannosalarias, Crossalarias, Atrosalarias, Salarias, and Glyptoparus. These genera share a distinctive dentary bone that forms an open capsule as a result of the loss of the anterodorsal bony wall. The anterodorsal bony wall of the dentary is present in all other blennioid fishes, but is only poorly developed in the salariin genera Hirculops and Rhabdoblennius. With the exception of one of about 45 species ( $V$. G. Springer, 1971 and pers. comm.) of Ecsenius, members of the Alticus group have a very high number of premaxillary teeth (55 to almost 400 ) and dentary teeth (39 to about 300 ) as adults. The remaining salariin genera have 17-50 premaxillary teeth and 16-38 dentary teeth. All other blenniids typically have tooth counts less than or in the lower end of the range found in the Salariini.

The relationships of the remaining salariin genera (the Rhabdoblennius group), Cirrisalarias, Medusablennius, Mimoblennius, Litobranchus, Antennablennius, Alloblennius, Hirculops and Rhabdoblennius, are unresolved. The occurrence of lower tooth counts in these genera than in the Alticus group cannot be used to infer relationships, as this is the plesionurphic condition. It is not known whether they form a monophyletic group. A comprehensive analysis of the interrelationships of all salariin genera is needed. 


\section{Node I Group}

Although generic interrelationships within the Alticus group are unresolved, the genera referred to here as the node I group appear to represent a monophyletic unit. Springer (1968b) suggested these genera were related because they all have the terminal anal pterygiophore supporting two rays. Relationships within the node I group are presented in the cladogram in Figure 1. Monophyly for this group, comprising the genera Istiblennius, Entomacrodus, Stanulus, Pereulixia, Scartichthys, Ophioblennius, Exallias, and Cirripectes, is supporteu by three derived characters at node I: (1) last pleural ribs borne on first or second caudal vertebral centrum (this is usually centrum 11 or 12); (2) terminal anal pterygiophore bearing two rays; (3) each dentary of ophioblennius stage larvae with a large recurved canine positioned midlaterally on each dentary.

The node I group, with the exception of Stanulus, consistently has the last pleural ribs borne on the first or second caudal vertebra. Stanulus (some specimens rarely have them on the first caudal vertebra), the "Blenniini," and other blenniids typically have the last pleural ribs borne on the last precaudal vertebra. Among the salariin genera, only Alticus, Atrosalarias, and Salarias contain one or more species that usually have the last pleural ribs borne on a caudal vertebra, but each of these genera also has members with the last pleural ribs borne on a precaudal vertebra. Dodekablennos also has the last pleural ribs borne on a caudal vertebra and may be the sister. group to the node I group, but the unavailability of cleared and 
stained material leaves its status uncertain. The condition of the last pleural ribs borne on a caudal vertebra is considered apomorphic, while the last pleural. rios borne on a precaudal vertebra is plesiomorphic. Within the node I group, only Stanulus typically has the last pleural ribs on the last precaudal vertebra (Springer,1968a). Although this character argues for the exclusion of stanulus from this clade, additional characters at node II support its inclusion on the basis of parsimony. Some species of the genus Istiblennius have the last pleural ribs borne on a precaudal vertebra. This and other characters suggest that Istiblennius, as presently defined, way be polyphyletic. A complete revision of Istiblennius is needed to define its limits.

The second character supporting monophyly for the node I group is the presence of two rays on the terminal anal pterygiophore. Although Smith-Vaniz and Springer (1971) stated that Praealticus frequently has the last anal-fin ray divided through the base, I found this to be an extremely rare and probably exceptional condition in Praealticus. Istiblennius shows variation in this character that appears to be species specific. Many Istiblennius species have the last two anal rays on one pterygiophore, whereas other species have the last two rays borne on separate pterygiophores. Most genera in the node I group show some variation in this character, as discussed by Springer (1967), but they usually have the last anal pterygiophore bearing two distinct rays. Other blenniids and many blennioids (including the Chaenopsidae, Labrisomidae, and Ophiclinini) have the last anal pterygiophore bearing a single ray. The presence of two rays on the last anal 
pterygiophore is considered the derived condition for blenniid fishes, and supports monophyly for the node I group. I should note that the last anal ray split to base is pleisomorphic for the Perciformes. Among blennioids, at least the Blenniidae (except the node I group) and Chaenopsidae do not have the last ray split. Tire simplest explanation for the presence of the split ray in the node I group is that it is a reversal and, thus, is apomorphic at this node.

The third character supporting monophyly for the node I group is a characteristic of the ophioblennius stage larvae. The ophioblennius stage larvae of each of the genera in the node I group, except Pereulixia, for which a larval stage has not been found, have two posterolaterally directed, large, recurved canines located about midlaterally on each dentary. Istiblennius is problematical in that an ophioblennius larval stage is known only for members of the $I$. gibbifrons species complex (Smith-Vaniz and Springer, 1971). If the absence of this larval stage in other Istiblennius species is real and not a collecting artifact, then the genus as presently defined may be polyphyletic. No other blennioid larvae possess these large hooked canines midlaterally on each dentary. Larval Nemophini have large canines in the jaws, but these teeth are not hooked. The presence of large hooked teeth is considered a derived condition and is evidence for monophyly of the node I group. Although larval Pereulixia are not known, the presence of several additional characters, discussed later in this paper, places this genus in the node I group on the basis of parsimony. 
Genus Istiblennius

The genus Istiblennius as defined by Smith-Vaniz and Springer (1971) (or some part of the genus including the Istiblennius gibbifrons species complex) appears to be the sister group of the remaining members of the node I group. As indicated above, I know of no character that supports a hypothesis of monophyly for the species included in Istiblennius. The genus is presently being revised.

\section{Node II Group}

This group of genera (Entomacrodus, Stanulus, Pereulixia, Scartichthys, Ophioblennius, Cirripectes, and Exallias) shares two derived characters: (1) each anal-fin spine of adult males enveloped in a fleshy rugosity and (2) posteriormost branchiostegal ray with an elongated proximal portion extending well onto the posterior ceratohyal and passing Jorsad of the proximal end of the preceding branchiostegal ray (Figure 6C).

In addition to the node II group, adult males of Nannosalarias Eypically have fleshy rugosities enveloping each anal-fin spine. As discussed in the Blenniidae section, this condition is a synapomorphy of the Blenniidae. Based on my hypothesis of relationships within the Salariini, the occurrence of anal-fin spine rugosities in the node II group must be a secondary derivation because the rugosities have been lost at the hypothesized node at which the Alticus and Rhabdoblennius groups diverge. Inasmuch as the genera of the node II group and 
Figure 6. Left lateral view of branchiostegals and associated bones. (Anterior toward left.) A-fifth and sixth branchiostegal rays extending about equally onto ceratohyals (Crossosalarias macrospilus). B-sixth branchiostegal ray extending slightly dorsad of fifth (Stanulus seychellensis). C-sixth branchiostegal extending dorsad of and anterior to fifth (Cirripectes perustus). Abbreviations:

AC-anterior ceratohyal; DH-dorsal hypohyal; PC-posterior ceratohyal; $\mathrm{VH}$-ventral hypohyal. 


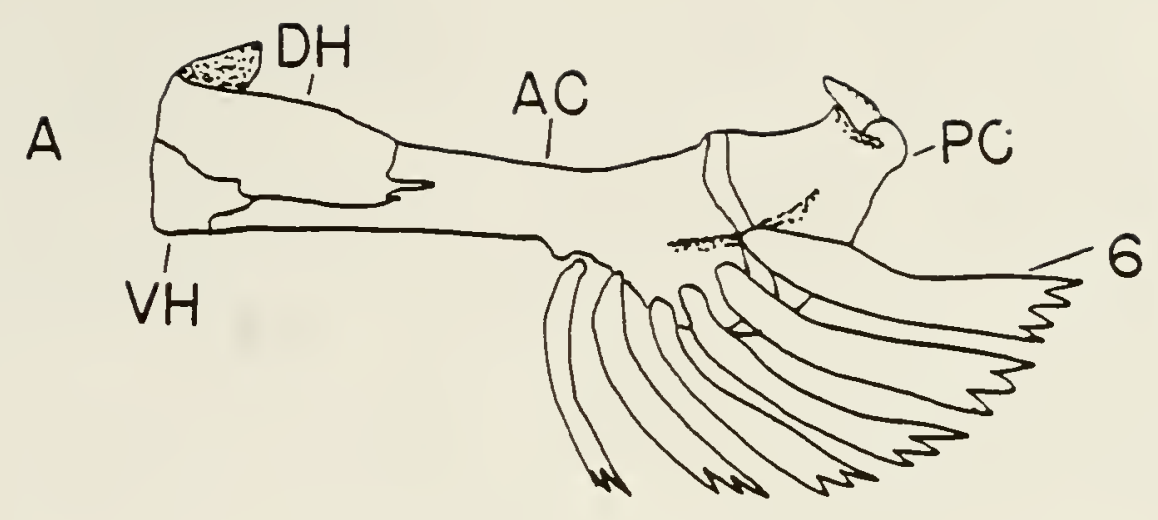

B

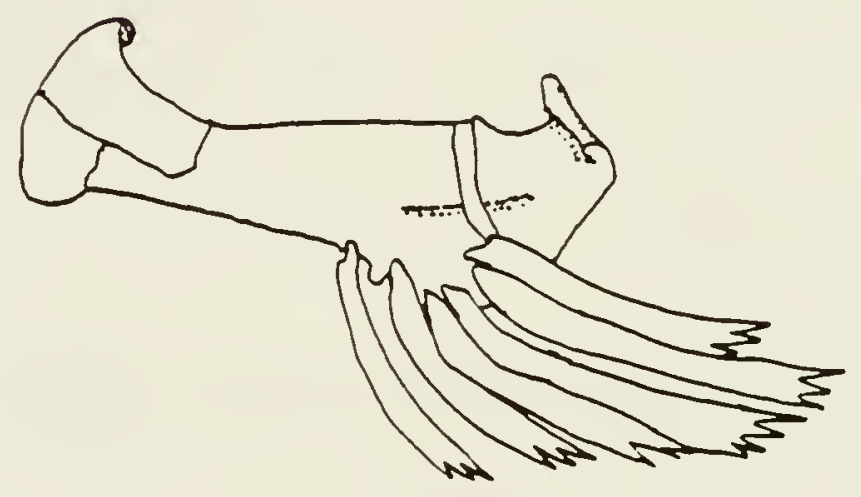

c

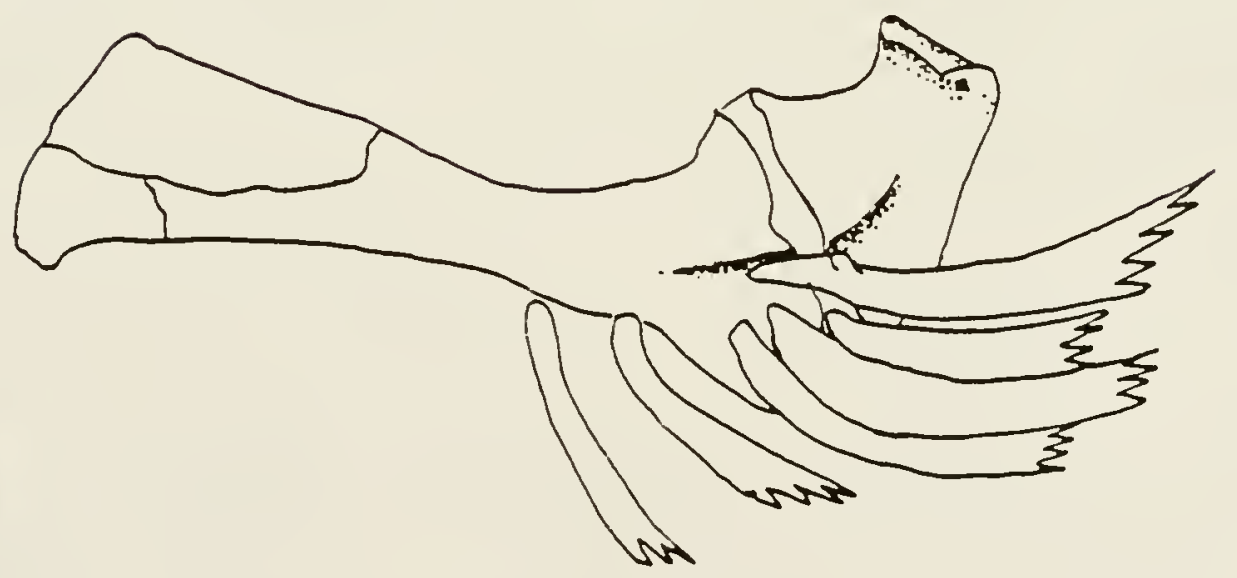


Nannosalarias do not share other characters defining the "Blenniini," the most parsimonous explanation is that this apomorphic character is either independently derived or convergent. Within the Alticus group, the rugosities appear to have been independently derived in Nannosalarias because it does not share the other characters defining the node I group. I interpret the presence of fleshy anal-fin spine rugosities as a synapomorphy of the node II group at this level of analysis.

The sixth (=posteriormost) branchiostegal ray, although variously shaped among blenniid genera, has two distinctive conditions associated with the development of the proximal portion (herein referred to as the head) that overlaps the posterior ceratohyal: (1) head of posteriormost and preceding branchiostegal rays extending equally far onto"the posterior ceratohyal (Figure 6A) or (2) head of posteriormost ray extending as much as three to four times as far onto posterior ceratohyal as preceding ray (Figure 6C). Condition 1 is the typical condition found in other salariin and blennioid fishes and is considered the plesiomorphic state. The node II genera, except Pereulixia, which has condition 1 , share condition 2 . The most parsimonious explanation for the occurrence of condition 1 in Pereulixia is that it is a reversal to the plesiomorphic condition. In condition 2, the elongated head of the sixth branchiostegal ray has an anterodorsally directed component projecting over the dorsal tip of the preceding ray(s) and a posteriorly directed spur sometimes 
extending along a slight groove in the posterior ceratohyal. There is a ligamentous connection of the elongated portion of the branchiostegal head to the posterior ceratohyal. Condition 2 does not occur in other blennioid fishes and, thus, is a synapomorphy of the node II genera.

The head of the sixth branchiostegal ray in Stanulus is not as extensively developed as in the node II genera. In Stanulus, the head is only slightly elongated in an anterodorsal direction, reaching a point just above the preceding ray (Figure 6B). Stanulus is a genus of small species and the condition might be neotenic. This condition is interpreted as being = modification of condition 2 .

\section{Genus Entomacrodus}

I know of no uniquely derived character that will define the genus Entomacrodus as a monophyletic taxon. Entomacrodus has vomerine teeth, but vomerine teeth are also present in Pereulixia and some Stanulus.

Smith-Vaniz and Springer (1971) and Springer (1967, 1968a) stated that Entomacrodus is most closely related to Stanulus, based on their similarity in overall appearance. I have found no synapomorphy to support this hypothesis.

\section{Node III Group}

This group comprises the genera Stanulus, Pereulixia, Scartichthys, Ophioblennius, Cirripectes, and Exallias. I have found only one character that defines this assemblage. These genera, except 


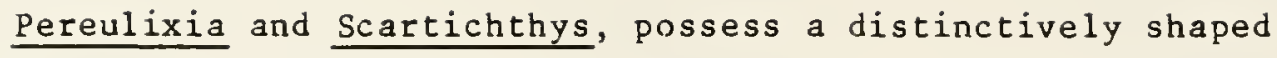
infrapharyngobranchial bone (Figure 5G-K). The anteromedially positioned flange of the infrapharyngobranchial plate (either a fused complex of one or more of infrapharyngobranchial bones 2,3 , and 4 , or a loss of one or two of these bones) has one or two large foramina, giving the bone a doughnut-shaped appearance (Figure 5). This condition appears to be unique and defines these genera as a monophyletic group.

The infrapnaryngobranchial plate of Pereulixia and Scartichthys is typical of othir salariin genera (Figure 5F; see previous discussion for the Salariini) and does not show the modifications present in the other members of this group. This condition is interpreted as a reversal based on parsimony.

\section{Genus Stanulus}

Stanulus, the hypothesized sister group of the other node III genera, has a distinctive ophioblennius stage larva that is much smaller than the larvae (at an equivalent stage of development) of other genera in the node I group. Similarly, adult Stanulus are smaller than other members of this group (except Entomacrodus thalassinus and E. strasburgi). In addition, stanulus has scalelike flaps along the anterior portion of the lateral line. Pereulixia and several species of Cirripectes have similar scalelike flaps associated with the anterior lateral line pores, but these genera possess additional characters that argue for an independent derivation of the flaps in each genus. 


\section{Node IV Group}

The node IV group comprises Pereulixia, Scartichthys, Ophioblennius, Cirripectes, and Exallias. Three derived characters define this group: (1) transverse row of cirri extend across nape (Figure 7); (2) symphyseal portion of the premaxillary bone connected to remainder of premaxillary by a slender bridge of bone beneath the anterior edge of the base of the ascending process, symphyseal portion is free posteriorly (Figure 8B); (3) lacrimal with an elongate antera: antral extension that reaches ventrally as far as the lower edge of the maxillary bone and has a sensory canal opening at or near its ventral edge (Figure 9B).

A distinctive transverse row of cirri across the nape in the node IV genera manifests itself variously in the different genera. Although some genera of the Labrisomidae (sensu George and Springer, 1981) also have a transverse row of nuchal cirri, this character is considered to be convergent, as these groups are clearly not closely related. The different patterns of this transverse row of cirri will be discussed later for each genus or group of genera.

The distinctive shape of the body of the premaxillary bone in the node IV group appears to have resulted from a loss of bone in the concavity beneath the base of the ascending process. The posterior tip of the free symphyseal portion is tightly connected by ligaments to the posterior edge of the base of the premaxillary ascending process and the anterior edge of the maxillary bone. The base of the ascending process straddles the unossified concavity in the body of the 
Figure 7. Dorsal views of nuchal cirri. (Anterior toward top.) A-type A, Cirripectes kuwamurai. B-type B, C. variolosus. C-type C, . gilberti. D-type D, . perustus. E-type E, C. alboapicalis. F-type F, C. jenningsi. G-type G, C. vanderbilti. H-Pereulixia kosiensis (dashed line indicates cirri not illustrated). I-Exallias brevis. J-Scartichthys viridis. 


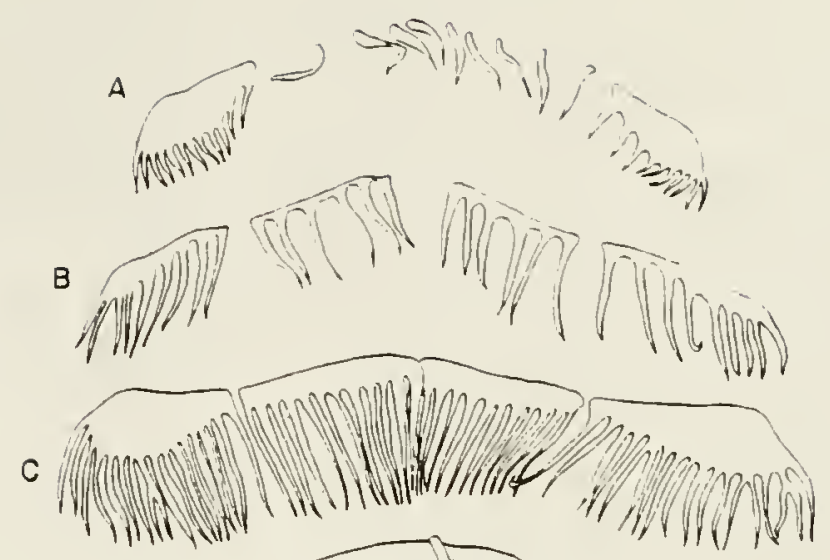

D

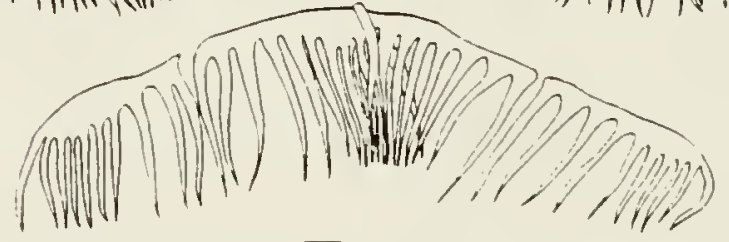

E
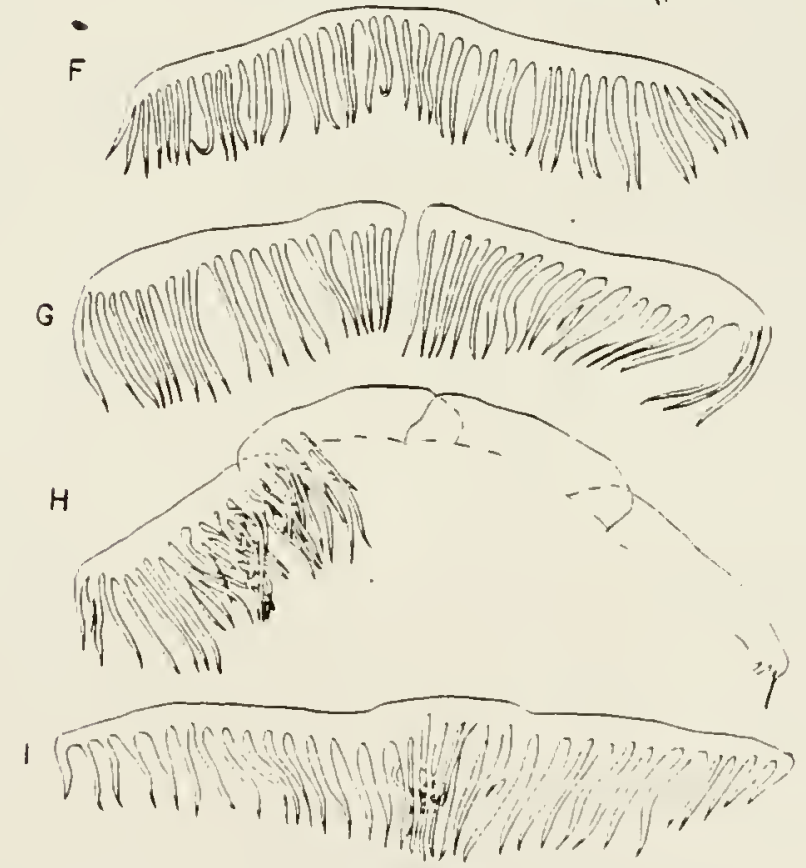

J

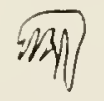

$\sqrt{(4 x) ! n}$ 
Figure 8. Dorsal views of left premaxillary bones (ascending process removed from each bone). (Anterior toward top.) A--Alticus saliens. B--scartichthys gigas. 

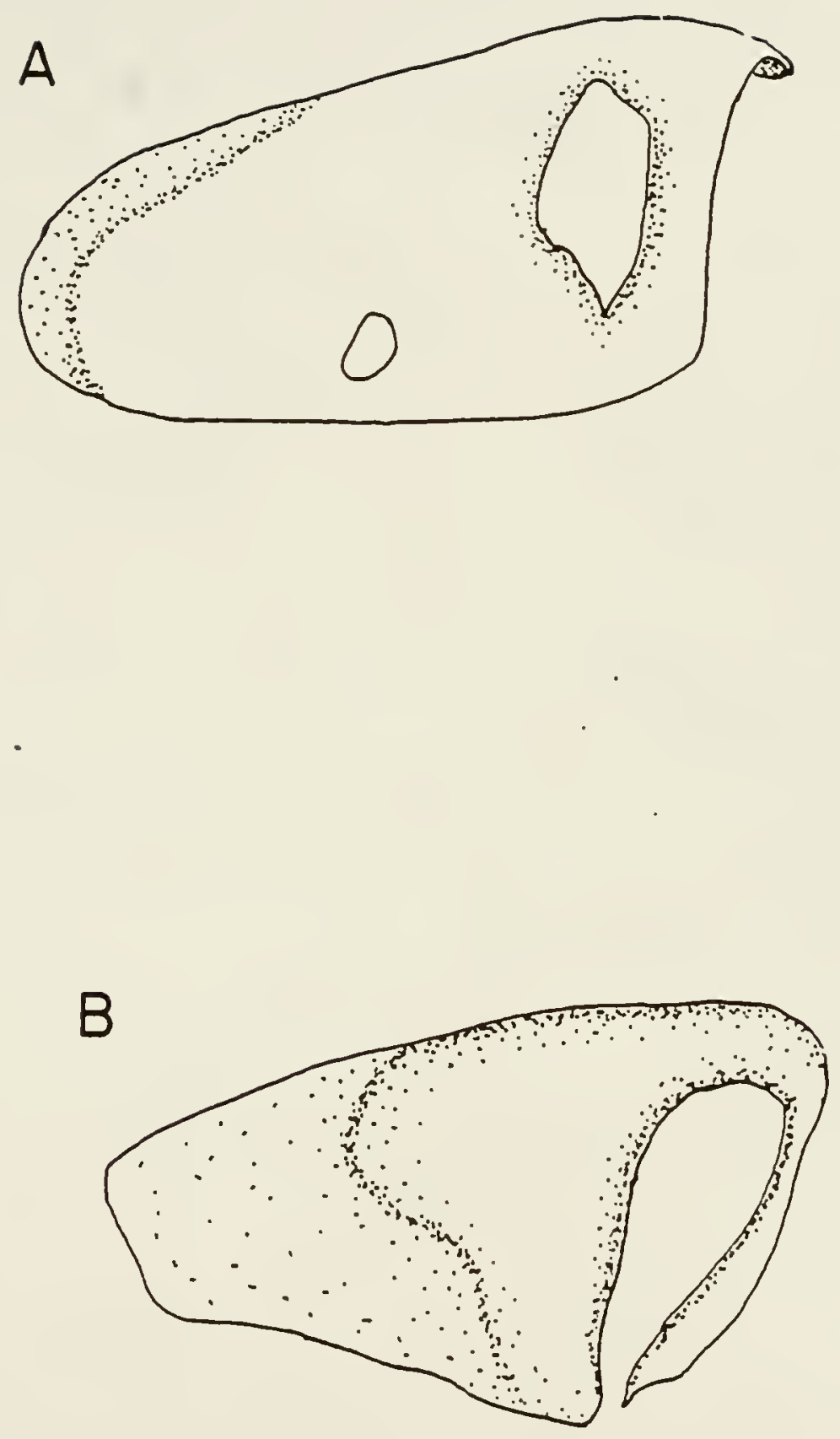
Figure 9. Left lateral views of left lacrymal bones. (Anterior toward left.) A-pores exit near main canal (Alticus saliens). $\mathrm{B}$-branch of canal almost reaches ventral margin of bone (Scartichthys gigas). 

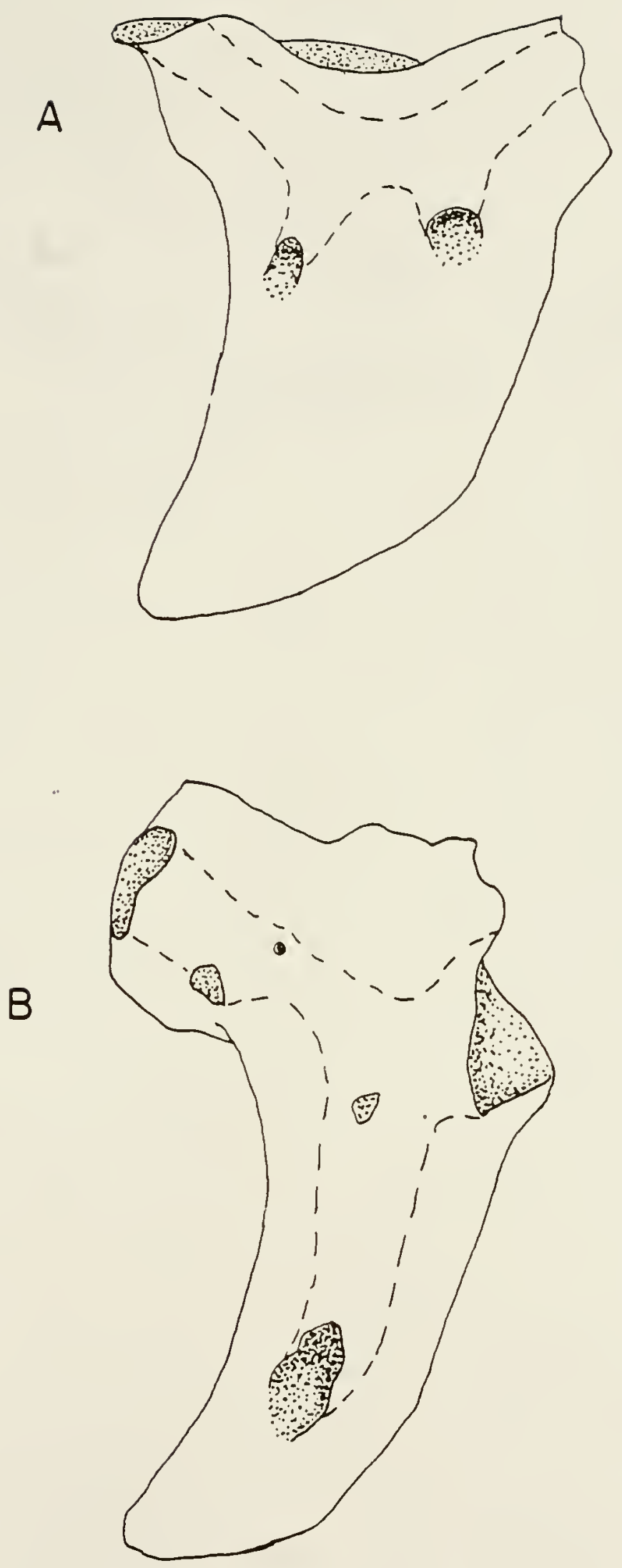
premaxillary bone and appears to serve as a point of attachment for connective tissue holding the teeth in place. Other genera of the Blenniidae either do not have an open gap in the body of the premaxillary bone, or, if there is an opening, there is an anterior and posterior bony connection to the main body of the premaxilla (Figure 8A). The loss of the posterior bony connection between the symphyseal portion and the main body of the premaxillary is a synapomorphy of the node IV group.

The distinctively shaped lacrimal (Figure 9B) of the node IV group is not found elsewhere in the Blenniidae. In most other salari in genera, the lacrimal is much shorter and broader than it is in the node IV group, and the ventrally directed sensory canal(s) exits from the bone at a point relatively close to the main infraorbital canal. The slender, elongate, anteroventrally directed extension of the lacrimal is a synapomorphy of the node IV genera. Ecsenius is exceptional in having an elongate lacrimal (Springer, 1968b:pl.9), but the ventral sensory canal exits relatively r.lose to the main infraorbital canal.

\section{Genus Pereulixia}

The genus Pereulixia forms the sister group of the rode $V$ group and is distinctive in having a nuchal cirri arrangement consisting of four slightly overlapping grüps of cirri. The cirri are frequently complex, with multiple branches arising from a single base. The skull 
Figure 10. Left lateral view of supraoccipital region of skull of Pereulixia kosiensis illustrating well-developed crest. 
47

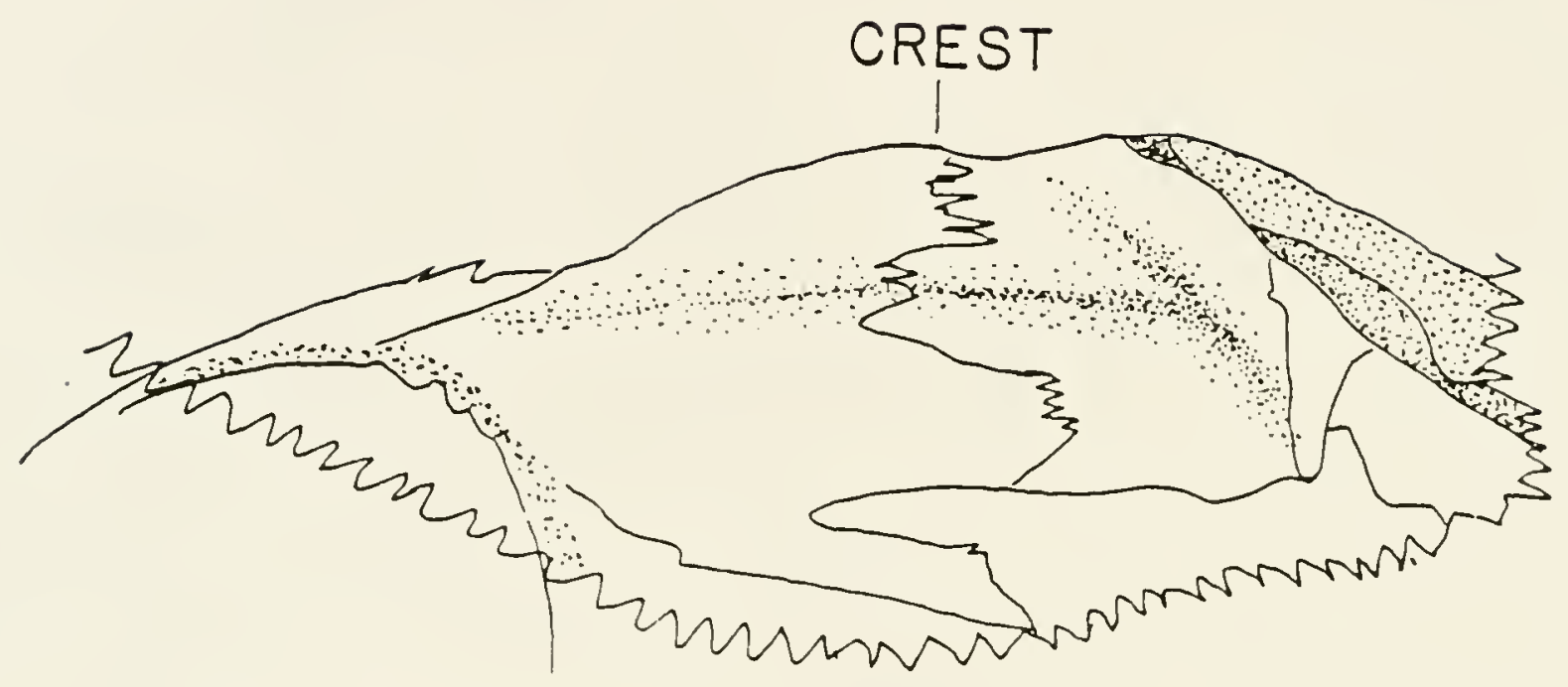


of Pereulixia has a high bony crest extending anteriorly from the dorsalmost edge of the supraoccipital bone to a point above the posterior edge of the orbits (Figure 10). The crest is formed from dorsal expansions of the frontal and parietal bones, each of which comprises about half of the crest and are firmly sutured to each other. Other Salariini may have short bony crests on the skull, but the crest does not reach the height of the dorsalmost tip of the supraoccipital bone. Even salariin taxa with well-developed fleshy crests have only low bony crests on the skull. Both of these characters are considered derived. In other blenniid tribes, a large bony crest is present in large Hypleurochilus and Omobranchus aurosplendidus. These occurrences are considered independent derivations.

Pereulixia"has larger (thus fewer) dentary incisors than premaxillary incisors. Exallias has a similar dentition, but has more incisors in both jaws. Ecsenius has dentary incisors larger than premaxillary incisors, but there are fewer incisors. This character of dentary incisors larger than premaxillary incisors is considered to be a derived condition. Based on the characters defining the node III and $V$ groups, I hypothesize independent derivations in these genera.

The relationship of Pereulixia to other salariin genera is somewhat tentative. Although it shares several characters with the node IV group, its placement here is only slightly more parsimonious (two fewer steps) than placing it as the sister group to Istiblennius and the node II group. 


\section{Node V Group}

The node $V$ group includes Scartichthys, Ophioblennius, Cirripectes, and Exallias. This assemblage is defined by the following five synapomorphies: (1) fourth epibranchial with two distinct processes located on the dorsal side near the midpoint of the shaft of the bone, one process directed anterodorsally and the other posterodorsally (Figure 4); (2) fifth ceratobranchial with an expanded flange directed ventrally and posteriorly from the main tooth-bearing portion of the bone, flange with a straight vestical margin posteriorly that forms a sharp right angle where it meets the relatively straight ventral margin of the flange (Figure 11); (3) each premaxillary bone of ophioblennius stage larvae with one or two large recurved canines near symphysis of premaxillaries; (4) each dentary bone of ophioblennius stage larvae with one or two large recurved canines at symphysis of dentaries; and (5) dorsal-fin membrane attached to caudal fin. The above characters 1 through 4 are present in (and exclusive to) all node $V$ genera and are synapomorphies of the group. Character 5 is a derived character but occurs homoplasiously.

No other blennioids have two distinct processes on the fourth epibranchial bone. The plesiomorphic condition is a single broad dorsal projection in this position on the fourth epibranchial and occurs in all blennioids except the "Blenniini" and node V group. There appear to be two derived conditions for the fourth epibranchial: two projections or no projections. The former condition is a synapomorphy of the node $V$ group and the latter a synapomorphy of the tribe "Blenniini." 
Figure 11. Left lateral views of left fifth ceratobranchial bones. (Anterior toward left.) A-Acanthemblemaria aspera. $B$-Chasmodes saburrae. C-Mimoblennius atrocinctus. $\mathrm{D}$-Hirculops cornifer. E-Alticus saliens. F-Scartichthys gigas. Abbreviations: $\mathrm{CN}$-constriction; $\mathrm{PF}$-posterior flange; UF-unconstricted flange; $V F$-ventral flange. 

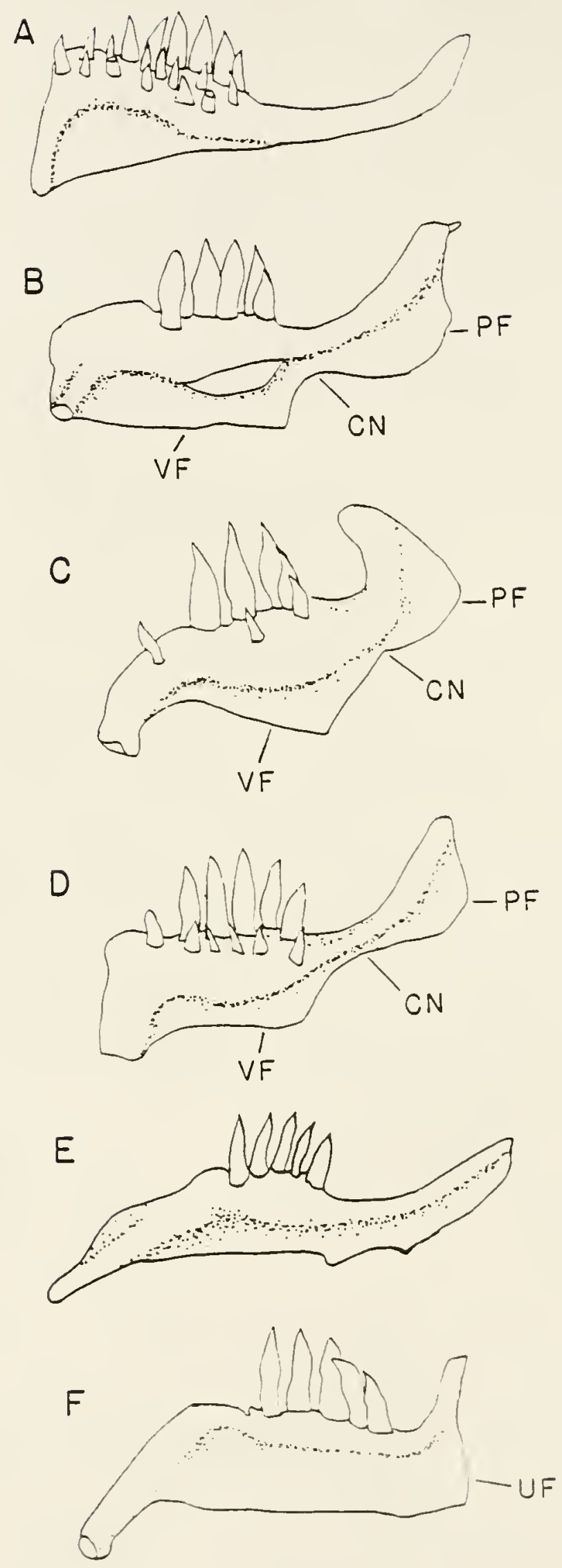
In the node $V$ group, the fifth ceratobranchial has a distinctive, unconstricted, ventroposteriorly directed flange (Figure $11 \mathrm{~F}$ ) that extends posteriorly to a point beyond the main body (tooth-bearing portion) of the bone. Alloblennius, Mimoblennius, Hirculops, and Litobranchus also have a posteriorly directed flange, but, in these genera, the flange is quite distinctive in having a rounded extension of the flange at the posterior edge and a constriction separating it from an angular process on the ventral edge (Figure 11C-D). Other salariin genera have a reduced posterior flange that does not project posteriorly beyond the dorsal limb of the fifth ceratobranchial (Figure 11E). Genera of the "Blenniini" have a rounded flange of bone on the posterior edge of the dorsal limb of the fifth ceratobranchial, another flange located ventrally below the tooth-bearing portion of the bone, and a distinct constriction between the flanges at the dorsoposterior edge of the tooth-bearing portion (Figure 11B). All other blennioids have a slender fifth ceratobranchial bone with a very narrow flange of bone on the ventromedial edge of the tooth-bearing portion (Figure 11A). The slender fifth ceratobranchial with a very narrow flange of bone is considered the plesiomorphic condition. The unconstricted, extensively developed flange of bone on the fifth ceratobranchial bone is a synapomorphy of the node $V$ group.

The third and fourth synapomorphies for the node $V$ group are dentitional characters of the ophioblennius stage larvae. The first of these is the presence of large, hooked canines at the symphysis of the 
premaxillary bones. The second character is the presence of large, hooked canines at the symphysis of the dentary bones. Watson (in Leis and Rennis, 1983) distinguished the genera Exallias, Entomacrodus, Cirripectes, and Stanulus from the Nemophini by their large, hooked canines in the jaws. Although Watson suggested that the hooked canines serve to identify salariin larvae, these dentitional characters define only the node $V$ genera. The genera of the node $V$ and $I$ groups share in having a large hooked canine midlaterally on each dentary, but, unlike the node $V$ group, other members of the node I group do not have hooked zmphyseal canines. These distinctive hooked symphyseal canines also are lacking in all other blennioid larvae; thus, I consider them to represent a derived condition.

Most members of the node $V$ group have the dorsal-fin membrane attached to the caudal fin at a point above or posterior to the caudal-fin base as adults. Most salariin genera have the dorsal-fin membrane attached to the caudal peduncle anterior to the caudal-fin base. Although the dorsal $f$ in is attached to the caudal fin in many other blennioids, this attachment is considered. to be apomorphic at this level of analysis, inasmuch as the dorsal fin is not attached to the caudal fin in other node I genera (except some species of Istiblennius). Although the dorsal-fin membrane is not attached to the caudal fin in Scartichthys and a few species of Cirripectes, I consider this condition to be either a reversal, or a secondary derivation, based on parsimony. 


\section{Genus Exallias}

Exallias, a monotypic genus, is the sister group of the node VI group and is distinctive among all blennioids in having the lateralmost of the nuchal cirri very short, with cirri increasing in length toward the nape.

Both sexes of Exallias lack dentary canines. Although some species of Istiblennius also lack dentary canines, all other genera in the node I group have them. The most parsimonious explanation is that the lack of dentary canines in Exallias is an apomorphy at this level of analys is.

Exallias differs from other members of the node $V$ group in having enlarged dentary incisors. Exallias usually has about three or four times as many premaxillary incisors as dentary incisors. Pereulixia has a similar dentition, but has fewer incisors on both $\mathrm{j}$ aws (Smith-Vaniz and Springer, 1971: table 2). Other members of the Salariini (see discussion of Ecsenius in Pereulixia section) have similar sized incisors in each $\mathrm{j}$ aw with up to twice as many premaxillary incisors as dentary incisors. I consider the possession of enlarged dentary incisors an apomorphic condition that has been independently derived in Exallias and Pereulixia.

\section{Node VI Group}

This group comprises Cirripectes, Scartichthys, and Ophioblennius and is defined by one synapomorphy, a large prominence at about the 
Figure 12. Posterior view of left maxillary bones. A--Alticus saliens. B--Scartichthys gigas. Abbreviation: MP--maxillary prominence. 

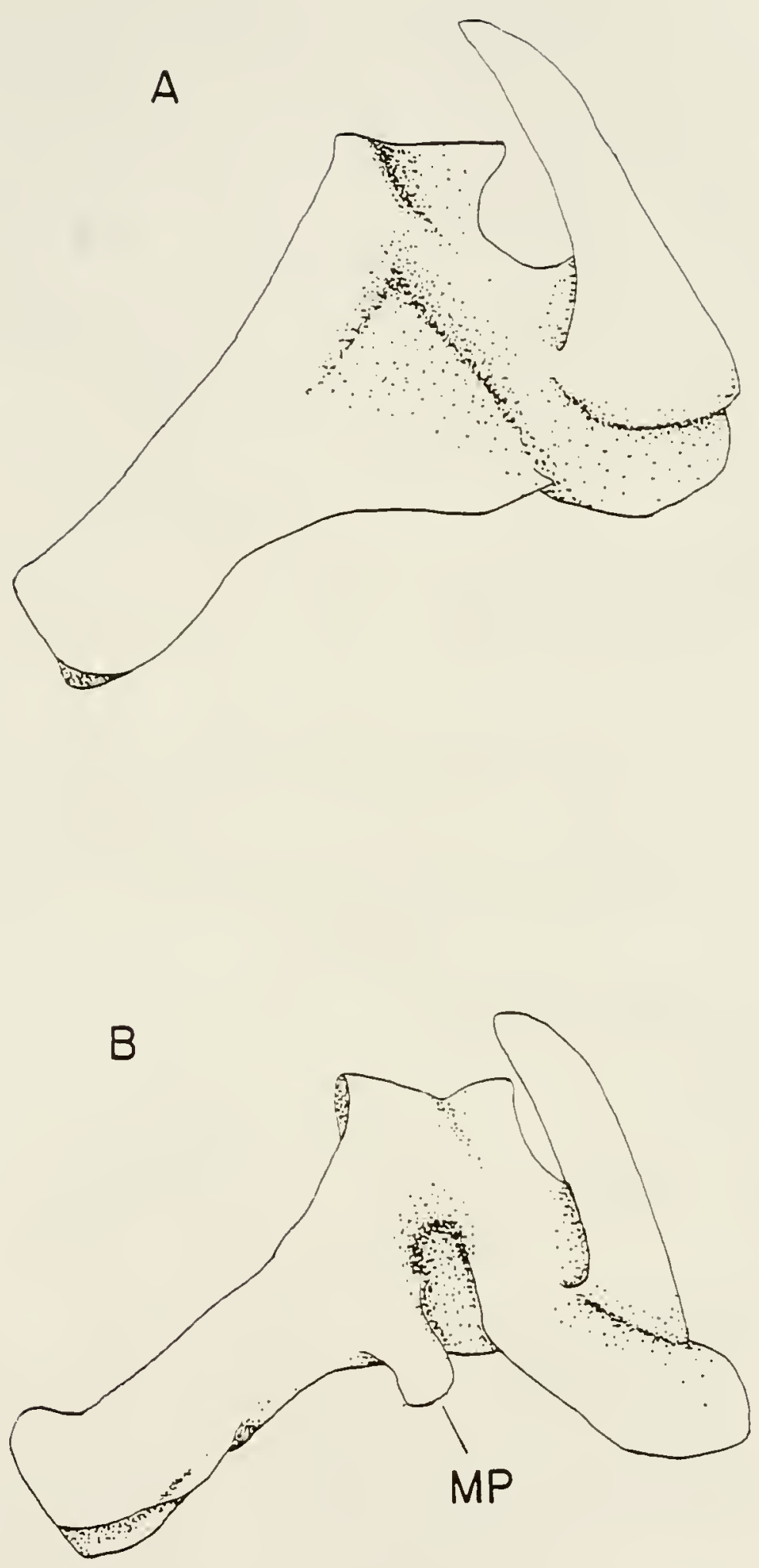
midpoint of the posteromedial edge of the maxillary bone (serves as the point of insertion for the primordial ligament).

The wel1-developed maxillary prominence ( $F$ igure 12B) is not present in other blennioid fishes, but, occasionally a slender flange in approximately the same position, or slightly anterior to this position, is present in other salariin genera. The presence of a wel1-developed maxillary prominence is a synapomorphy of the node VI group.

\section{Genus Cirripectes}

Cirripectes, sister group of the node VII group, contains a diverse assemblage of species. I have found only two synapomorphies that define Cirripectes: (1) distinctive nuchal cirri arrangement (Figure 7) and (2) adult male urogenital papilla with one or two tapering filaments associated with gonopore (Figure 13).

All Cirripectes have nuchal cirri that are atsut equal in length, typically simple, and arranged in a single row that may be divided into as many as four groups. When there are two groups on one side of the nape, the two groups do not overlap where their bases meet. In all but two species, there is a small break in the row of nuchal cirri at the nape. This condition differs from that of all other node IV genera. 
Figure 13. Ventral views of male urogenital papillae. (Anterior toward top.) A--type I, Cirripectes perustus. B-type II, C. vanderbilti. C-type III with posteriorly positioned gonopore, $\underline{C}$. alboapicalis. D-type III with distally positioned gonopore. E-Scartichthys viridis. 
A

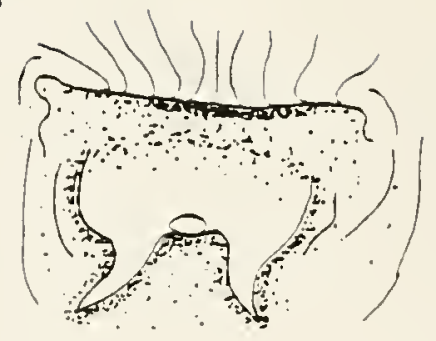

B

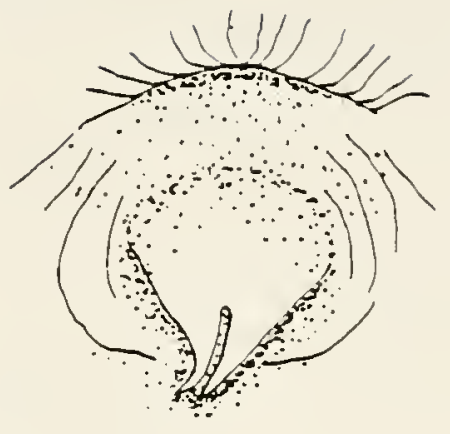

D

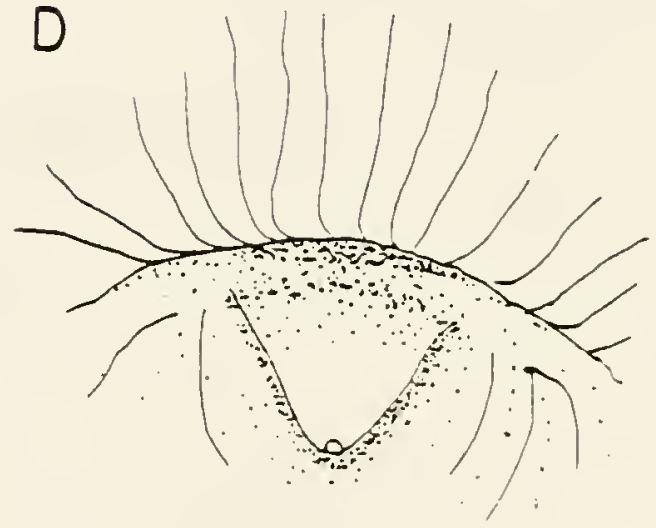

E

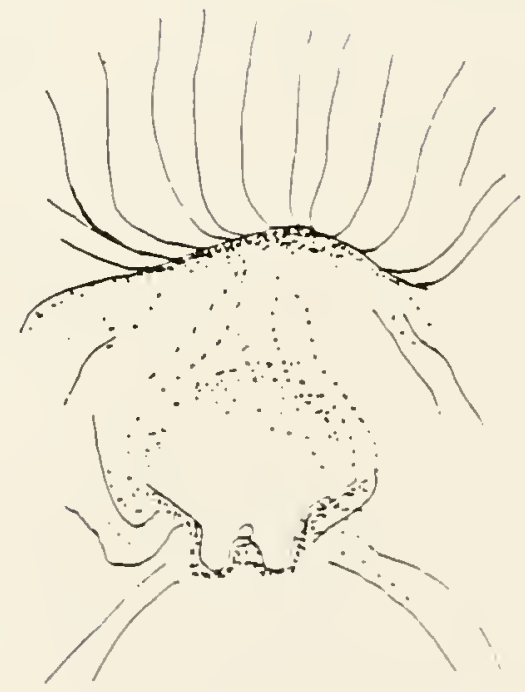


Exallias has the cirri increasing in length toward the nape and typically in a single continuous row across the nape. Scartichthys and Ophioblennius have a relatively short row of cirri on either side of the nape, with a wide gap separating the two rows. Pereulixia has four groups of nuchal cirri with overlapping bases. Large Pereulixia specimens usually have the cirri extensively branched, sometimes appearing as a series of palmate cirri. Because the nature and arrangement of the nuchal cirri in Cirripectes is unique, I hypothesize that the Cirripectes condition is a synapomorphy for the species of the genus. No other apomorphic characters are shared by all mabers of the genus.

Adult males have one or two slender filaments associated with the gonopore (Figure 13A-C). These filaments are variously modified in several species groups within Cirripectes. In all Cirripectes species groups, except one, the filaments extend beyond the gonopore and taper to a point. The relatively long tapering filaments contrast with the short blunt-tipped filament(s) found in Exallias and the node VII genera. Other blennioids have a gonopore at the tip of a short papilla. I consider each of the following as a synapomorphy for its group: the single short blunt protuberance in Exallias, the two blunt protuberances in the node VII group, and the one or two slender pointed filaments in Cirripectes. 


\section{Node VII Group}

Scartichthys and Ophioblennius are included in the node VII group. Three synapomorphies define this group: (1) male genital papilla flattened anteroposteriorly, with two short blunt projections on either side of the gonopore (Figure 13); (2) transverse row of nuchal cirri in two widely separated groups (Figure 7); and (3) high number of caudal vertebrae (22-26). The distinctive shape of the male genital papilla does not occur in other blennioids and I consider it ro be a derived condition.

The nuchal cirri arrangement in these two genera varies among species, but always consists of a group of cirri (as few as two in some Ophioblennius) on each side of the nape with a broad gap between the groups. This condition is hypothesized to be a secondary modification of the condition characterizing the node $I V$ group and, as such, is a synapomorphy for the node VII group.

Although other blenniids have a high number of caudal vertebrae, al1 other genera included in the node IV group have 19 to 22 caudal vertebrae (only a few species of Cirripectes have as many as 22 ). At this level of analysis, I consider the high number of caudal vertebrae (22-26) to be a synapomorphy for the node VII group.

Genus Ophioblennius

Ophioblennius is distinctive among blenniids in having disconnected overlapping portions of the lateral line. The anterior section of the 
lateral line ends at a point above the posterior section of the lateral line near the middle of the body. The posterior section of the lateral line begins slightly anterior to and beneath the end of the anterior section of the lateral line and continues to the caudal-fin base (frequently as a series of short tubes).

As discussed by Smith-Vaniz and Springer (1971), adults of this genus have dentary canines that are as long as or longer than the pupil diameter. Other members of the node I group have much shorter canines (or none at all) as adults. These two characters are synapomorphies of the species of Ophioblennius.

\section{Genus Scartichthys}

The genus Scartichthys is distinctive in that the posterior part of the lateral line consists of about 18-21 (typically 21) short tubes, each with a pore at either end. Other members of the node IV group usually have from none to 10 lateral line tubes. The high number of lateral line tubes is a synapomorphy of this genus. 


\section{DISCUSSION OF PHYLOGENETIC ANALYSIS OF GENERA}

Although the results of my study are in many cases tentative, I have attempted to present all supportive data clearly and concisely. As this study is the first attempt to provide a phylogenetic analysis of any group within the Salariini, it is possible that my hypothesis of relationships will be altered as the taxa are analyzed in greater depth.

The only previous attempt to provide a hypothesis of relationships within the Salariini was a dendrogram based on overall similarity (Smith-Vaniz and Springer, 1971; reproduced here as Figure 14). My hypothesis of phylogenetic relationships (Figure 1) differs from Smith-Vaniz and Springer's (1971) dendrogram in the relationships of several genera. In my analysis, Istiblennius (based primarily on the I. gibbifrons species complex) is included in the node I group, whereas Smith-Vaniz and Springer (1971) placed it with Praealticus, Alticus, and Andamia. It seems likely that this difference results from the particular species of Istiblennius used in each analysis. I agree with Smitil-Vaniz and Springer (1971:50) who state that Istiblennius might be polyphyletic and, thus, both placements could be correct for a specific subset of Istiblennius species. A revision of Istiblennius is needed before its relationships can be assessed adequately. 


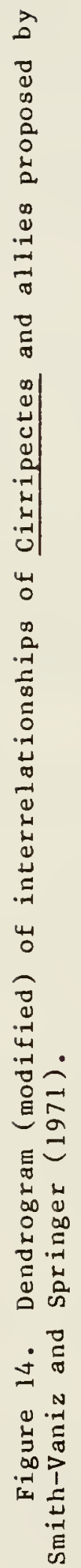




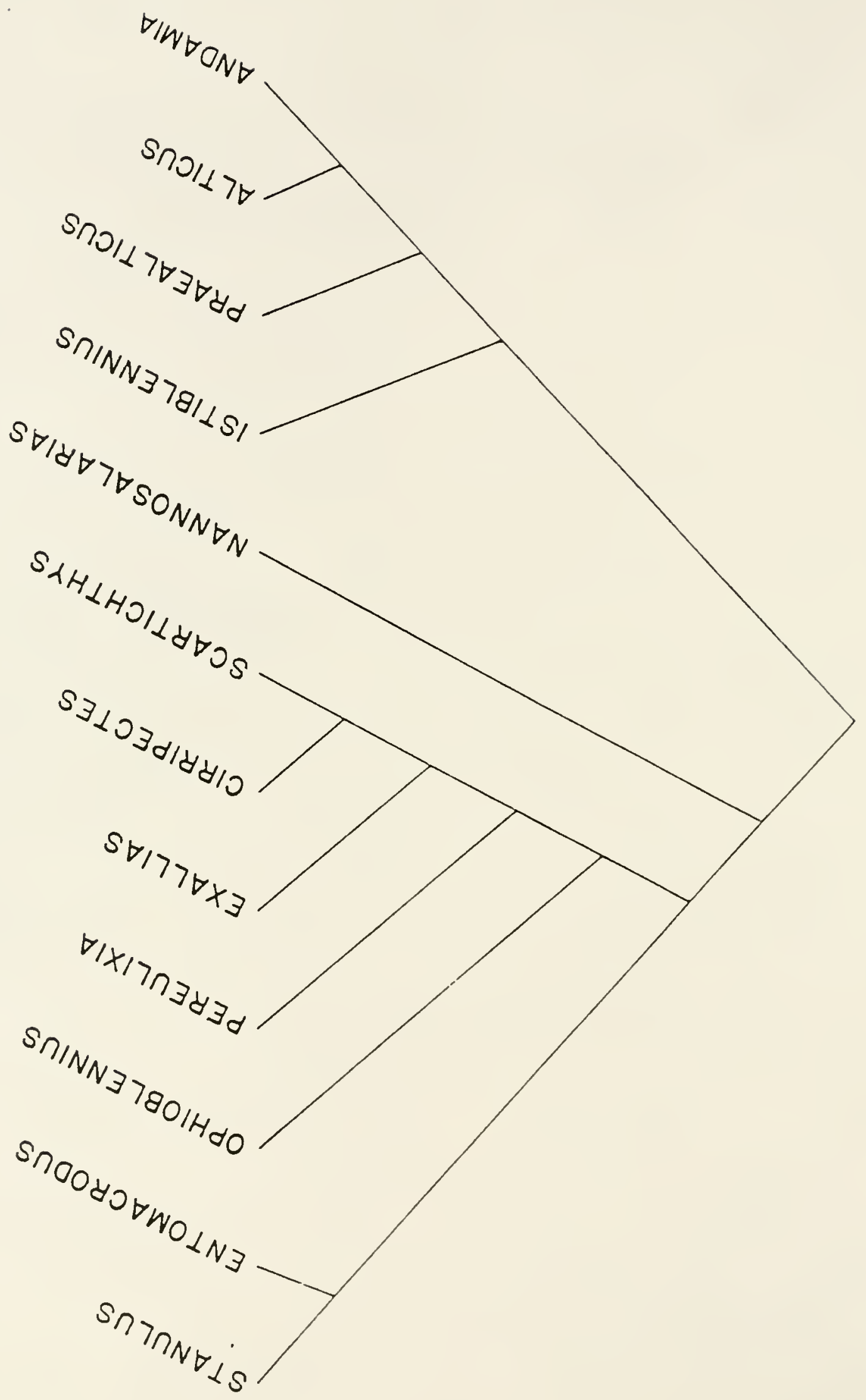


Smith-Vaniz and Springer (1971) considered Nannosalarias to be the sister group of my node II group. As I did not treat the interrelationships of the members of my Alticus group, outside of my node I group, it is possible that Nannosalarias is the sister group of the node I group. Further study of the Salariini is needed to determine the interrelationships of the remaining genera in the Alticus group.

Smith-Vaniz and Springer (1971) considered Stanulus and Entomacrodis to be sister genera that together form the sister group of my node IV zroup. They grouped Entomacrodus and Stanulus on the basis of overall similarity, but did not provide a specific character to support the hypothesized sister group relationship. In my analysis, Entomacrodus is the sister group of the node III group, which includes Stanulus and is defined by the presence of a large foramen in the infrapharyngobranchial plate. Thus, stanulus appears to be more closely related to the node IV group than to Entomacrodus.

Smith-Vaniz and Springer (1971) also regarded Ophioblennius as the sister group of the other genera in the node IV group. They placed it in this position because it possesses a combination of unique and distinctive characters that they felt isolated it from the other genera. In a cladistic analysis, unique characters cannot be used to infer relationship or lack of relationship with taxa not possessing these characters. In my analysis, Ophioblennius and Scartichthys are sister genera, which together are the sister group of Cirripectes. My determination of the relationship of Ophioblennius to the other genera 
in the node IV groun is based on a suite of characters at nodes V, VI, and VII.

Although many of the characters I used to determine relationships among the genera in my node I group are homoplasiously distributed, a cladistic analysis is the preferred method because it specifies how a character is distributed and clearly illustrates weak and strong components of the hypothesis. A cladistic hypothesis is defined by a specific distribution of characters among taxa and can be tested by adding additional characters or reassessing the original characters. As a result of the relative ease of testing a cladistic hypothesis, it is possible that any given hypothesis of relationships will change when additional characters are added to the analysis. Because of the potential for a given cladogram to change when additional-characters are added, formal taxonomic names should be applied with care in order to minimize future nomenclatural confusion. 
PHYLOGENETIC RELATIONSHIPS OF CIRRIPECTES SPECIES

I present a hypothesis of relationships among species of Cirripectes in the cladogram in Figure 15. To avoid confusion I began numbering the nodes on this cladogram with the next available node number (VIII) from the generic cladogram (Figure 1). All references to nodes I through VII refer to groups on the cladogram in Figure 1. The characters defining Cirripectes (the basal node in Figure 15) as a monophyletic genus were given in the earlier account of Cirripectes and will not be repeated here. Characters defining the terminal taxa (species) are given in the species accounts in the systematic section of this paper.

Morphological characters vary little among species of Cirripectes. Most species are differentiated from each other by subtle differences in color pattern or slight differences in meristic or other characters. Because of the morphological similarity of the species, few characters are available for use in a phylogenetic analysis. The phylogenetic relationships I hypothesize herein should be considered a working hypothesis of interspecific relationships.

\section{Node VIII Group}

This group is hypothesized to be the sister group of the remaining

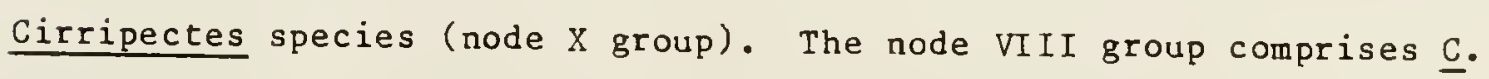




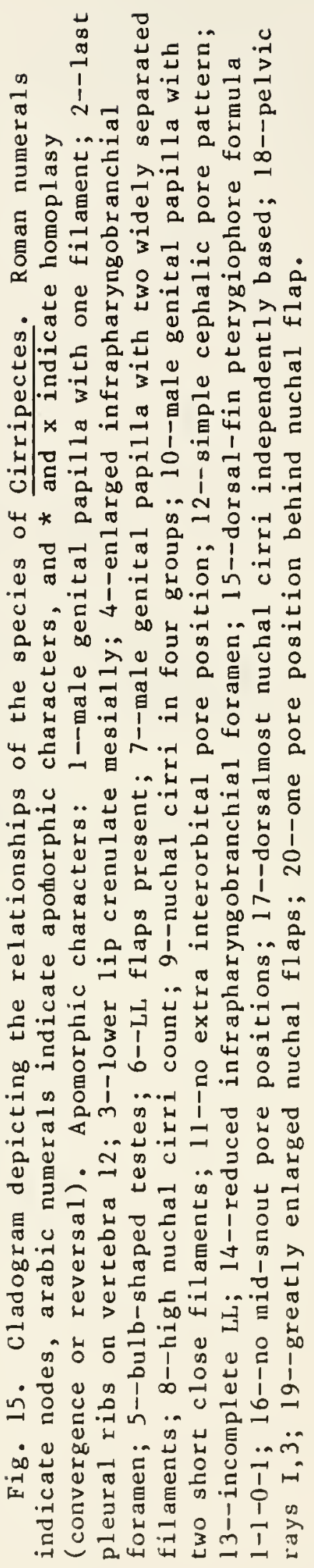




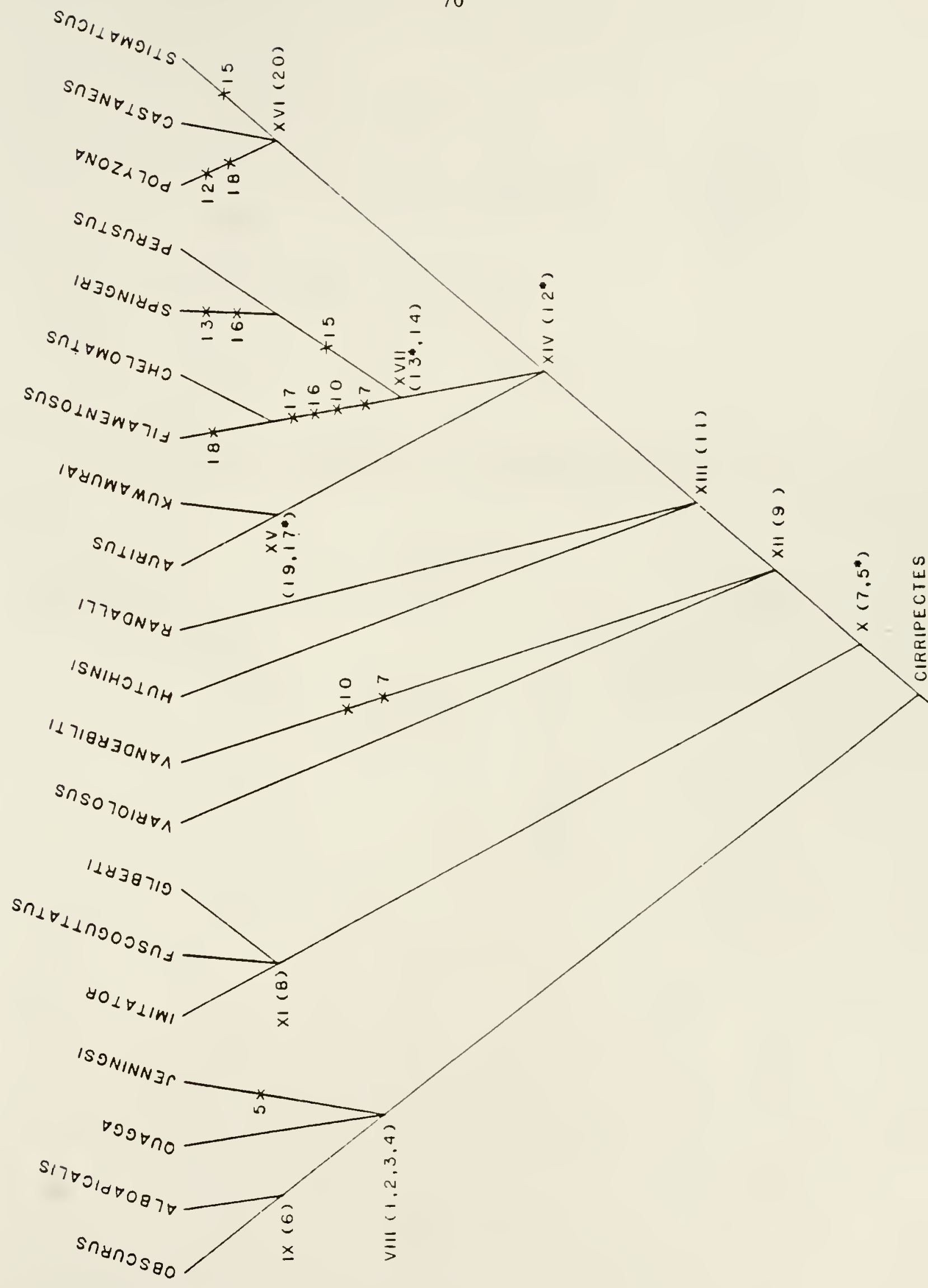


quagga, $\underline{C}$. jenningsi, $\underline{C}$. alboapicalis, and $\underline{C}$. obscurus. These four species are associated in an unresolved trichotomy between $\underline{\mathrm{C}}$. quagga, C. jenningsi, and the node IX group comprising ‥ alboapicalis and $\underline{\text { C. }}$ obscurus. Four synapomorphies define the node VIII group: (1) lower lip crenulate; (2) adult male genital papilla with a single slender filament, gonopore located at distal tip of filament or on posterior side near base of filament (Figure 13); (3) posteriormost pleural rib borne on second caudal vertebra; and (4) foramen in infrapharyngobranchial plate very large, reflecting a narrowing of the posterior part of the bony ring encircling the foramen (Figure 5).

Members of the node VIII group have distinct crenulae on the anteromedial edge of the lower lip. In other Cirripectes species, the medial portion of the lower lip has a smooth edge. Among the node I genera, a crenulate lower lip occurs only in certain species of Istiblennius and Cirripectes and, on the basis of parsimony, I consider these occurrences to be independent derivations of lower lip crenulae. Exallias has a deeply plicate lower lip, but does nnt have distinct crenulae on the anteromedial edge. The presence of distinct crenulae on the lower lip is a synapomorphy of the node VIII group.

The shape of the male genital papilla is apparently unique to the node VIII group and is considered a derived condition. The existence of several different adult male genital papilla morphologies in the node $V$ group of genera makes it difficult tc jetermine derived and plesiomorphic conditions. Three distinctive papilla morphologies are found among Cirripectes species. As none of these papilla morphologies 
are found in other blennioids, I interpret each of all three conditions as derived. Because each papilla morphology occurs only in Cirripectes, it is not possible to use o:1tgroup analysis to polarize the sequence of derivation of the three morphologies. Polarizing the character based on the most parsimonious distribution of derived characters for Cirripectes species results in a transformation series beginning with the short blunt papilla (plesiomorphic) leading to the following successively derived conditions: single slender filament, two widely separated filaments, and two short, closely appressed filaments. Although this technique can be considered circular reasoning, I tentatively accept this sequence, while realizing that an alteration of my cladogram could alter the polarity of this character.

The presence of the posteriormost pleural ribs on the second caudal vertebra is considered to be a derived condition that has arisen independently in some Scartichthys species and the node VIII group. I interpret the occurrence of pleural ribs on the second caudal vertebra as a synapomorphy of the node VIII group at this level of analysis.

In other members of the node $V$ group with a foramen in the infrapharyngobranchial bony plate, the posterior section of the ring of bone encircling the foramen is relatively thick. As the bony portion extends as far posteriorly in the node VIII group as in other members of the node $V$ group, it seems likely that the narrowing of the posterior section of the bony ring $\dot{*}$ inembers of the node VIII group resulted from a loss of bone from the interior edge of the posterior section of the ring. I consider the reduction in thickness of the 
posterior section of the ring of bone encircling the foramen to be a synapomorphy of the node VIII group.

\section{Node IX Group}

Cirripectes obscurus and $\underline{C}$. alboapicalis have scalelike flaps anteriorly on the lateral line. Stanulus and Pereulixia also have scalelike flaps. As discussed in the accounts of Stanulus and Pereulixia, this is an apmorphic character that has been independenciy derived in three different lines. I consider the presence of lateral-line flaps to be a synapomorphy of the node IX group at this level of analysis.

\section{Node X Group}

The node $X$ group comprises the remaining Cirripectes species, which are in the node XI and XII groups. The node $X$ group is defined by two apomorphic characters: (1) adult male genital papilla with two widely separated filaments (Figure 13) and (2) adult males with bulb-shaped testes (Figure 16).

Within blennioids, an adult male genital papilla with two slender filaments, one on eitier side of the gonopore, is unique to the node $\mathrm{X}$ group. This condition is mcdified in two groups of Cirripectes in that the filaments are reduced in length and closely appressed, but there are still two distinct filaments. The presence of two distinct filaments on the male genital papilla is a synapomorphy of the node $\mathrm{X}$ group. 
Figure 16. Ventral view of left testis of two Cirripectes species. (Anterior toward left.) A-elongate testis (Cirripectes obscurus). B-bulb-shaped testis (C. vanderbilti). 
A
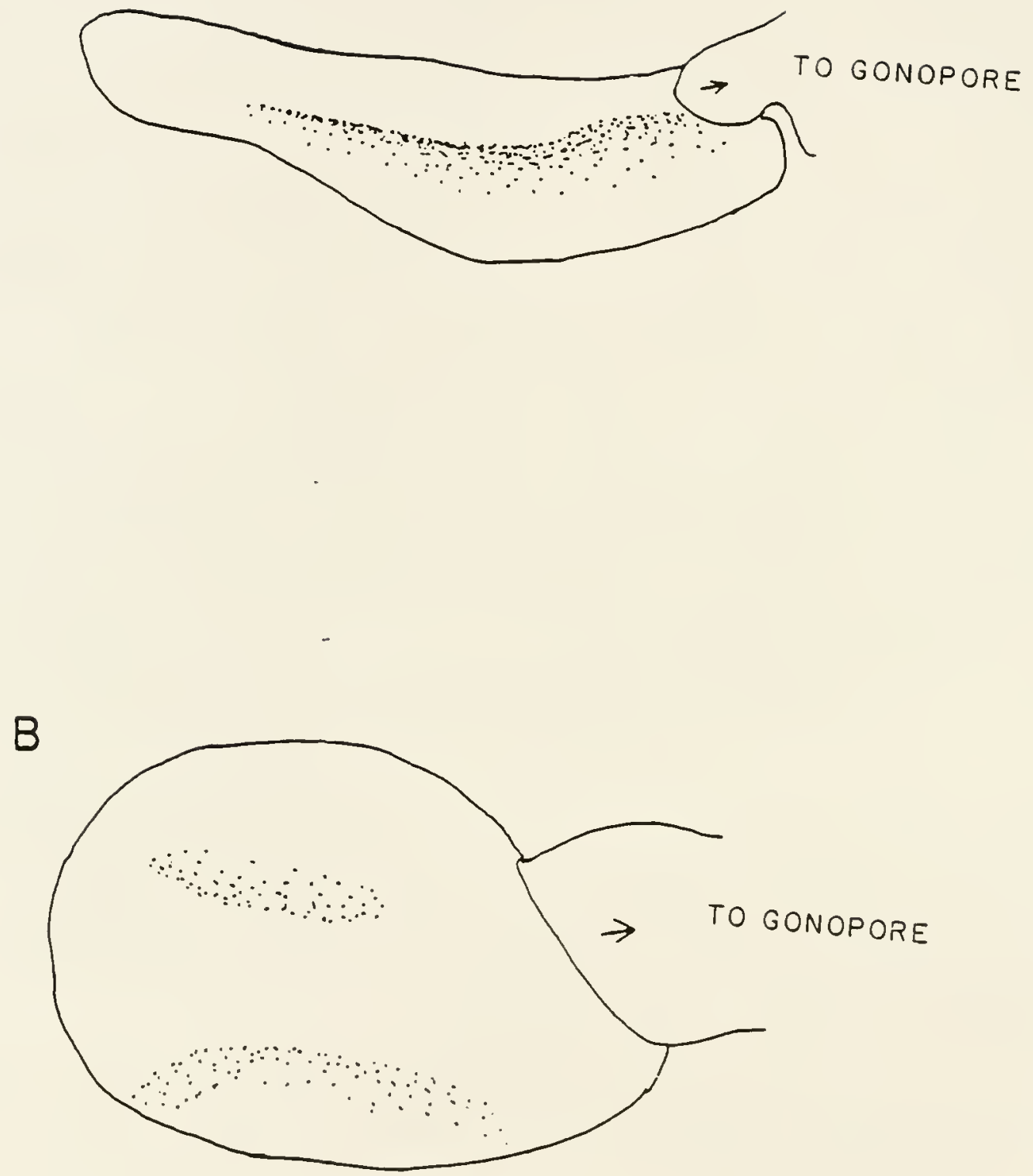
Adult males of the node $X$ group have bulb-shaped testes. All other blenniids examined have slender elongate testes. Bulb-shaped testes are a synapomorphy of the node $X$ group.

\section{Node XI Group}

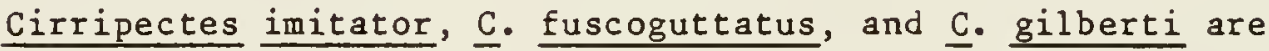
associated in a trichotomy at node XI. These three species share a distinctively shaped row of nuchal cirri (Figure 7). The nuchal cirri are arranged in four groups of cirri with tiue cirri in the ventralmost group on each side borne on a greatly enlarged flap. The node XI group also has a higher number of cirri than any other Cirripectes. The distinctive morphology of the row of nuchal cirri is a synapomorphy of the node XI group.

\section{Node XII Group}

The node XII group is associated in an unresolved trichotomy comprising Cirripectes variolosus, $\underline{C}$. vanderbilti, and the node XIII group. Members of the node XII group share a nuchal cirri morphology in which there is, on either side of the nape, a slightly expanded basal flap bearing the ventralmost cirri (Figure 7). The node XII species usually have four groups of nuchal cirri. This arrangement has been modified in $\underline{C}$. vanderbilti, where the two groups of cirri on 
either side of the nape are fused at their bases, reculting in two continuous rows of cirri with a short gap between the rows at the

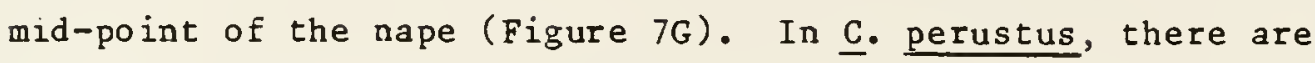
frequently three groups of nuchal cirri, one group forming a continuous row across the nape and a separate row ventral to the medial row on each side (Figure 7D). The node XV group has a fourth condition, in which the ventralmost group on either side is a greatly expanded flap bearing rudimentary cirri, with the cirri dorsal to this flap not connected basally (Figure 7A). Although there are four different modifications of the arrangement of nuchal cirri in the node XII group, all, except the highly modified condition in the node XV group, have a slightly expanded flap bearing the ventralmost nuchal cirri. I hypothesize that the general morphology (and secondary modificatons) of the nuchal cirri in the node XII group is a synapomorphy of the group.

\section{Node XIII Group}

The node XIII group is an unresolved trichotomy comprising Cirripectes hutchinsi, $\underline{C}$. randalli, and the node XIV group. The node XIII group lacks an interorbital pore (EIP) that is present in all other Cirripectes (Figure 17), Exallias, Ophioblennius, and Stanulus. The lack of an extra interorbital pore position is a synapomorphy of the node XIII group. 
Figure 17. Cephalic sensory pore patterns of Cirripectes. A and $B$ - complex pattern, dorsal and lateral views of Cirripectes imitator. $C$-simple pattern, lateral view of $\underline{C}$. perustus. Dashed lines indicate bases of cirri not illustraced. Abbreviations: AN-anterior nostril; EIP-extra interorbital sensory pore position; IFO-infraorbital sensory pore series; MD-mandibular sensory pore series; MSP-mid-snout pores; PBN-pore positions behind nuchal flap; PN-posterior nostril; POP-preopercular sensory pore series; SO-supraorbital sensory pore series; ST-supratemporal sensory pore series. Lines indicate first and last pores of each series. 

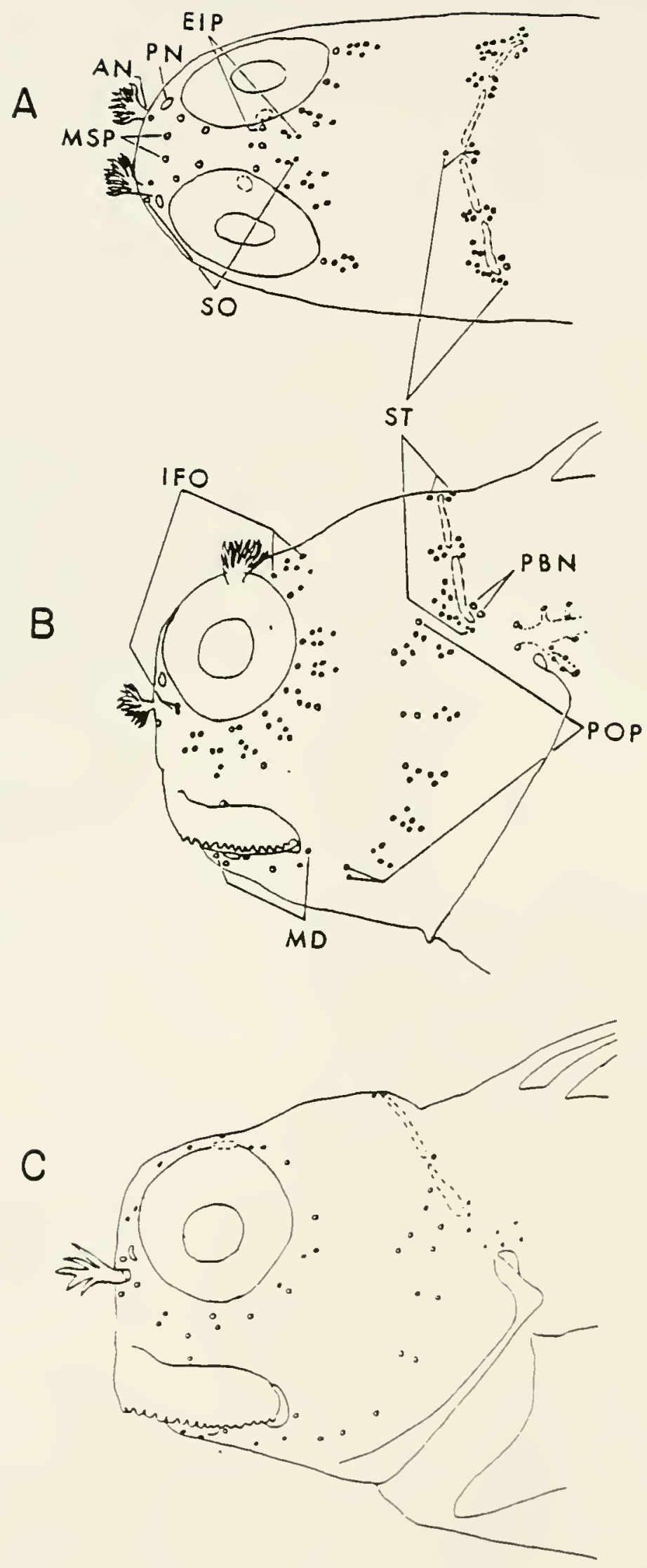


\section{Node XIV Group}

The node XIV group comprises the node XV, XVI, and XVII groups that are associated in an unresolved trichotomy. Members of the node XIV group, except Cirripectes polyzona, share a relatively simple cephalic sensory pore system, in which most pore positions have one or two pores (Figure 17C). Cirripectes polyzona and other members of Cirripectes have a complex cephalic sensory pore system, in which there are usually six or more pores at most positions (Figure 17A-B). Although a simple pore pattern is found in many other blennioids, Exallias and most Scartichthys have a complex pore pattern. The simple pore pattern is a symapomorphy of the node XIV group at this level of analysis. The complex pore pattern of $\underline{C}$. polyzona may be independently derived, as it shares another derived character with the node XVI group. An independent derivation of the complex pore pattern is the most parsimonious explanation. If the simple pore pattern is not considered an apomorphic character, the node XIV group collapses to form a polychotomy with the node XIII group.

\section{Node XV Group}

The node XV group comprises Cirripectes auritus and $\underline{C}$. kuwamurai, which share two apomorphic characters associated with the nuchal cirri 
(Figure 7): (1) larg? flap bearing rudimentary cirri present on each side of nape and (2) medial cirri (those located dorsal to nuchal flap) arising directly from skin, bases of cirri not connected by a low membrane.

The distinctive nuchal flaps of cirripectes auritus and $\underline{\mathrm{C}}$. kuwamurai are unique among blennioids. These flaps are a synapomorphy of the node XV group.

In the node $X V$ group and many specimens of Cirripectes filamentosus and $\underline{\text { C. }}$ chelomatus, the dorsalmost nuchal cirri arise directly from the skin. Uther Cirripectes and those related genera with a transverse row of nuchal cirri have the dorsalmost cirri on either side of the nape connected to each other by a low basal membrane. The most parsimonious explanation for the distribution of this character is that it has been derived independently in Cirripectes filamentosus and the node XV group. I consider the presence of independent1y based cirri to be an apomorphic character that provides additional evidence for the monophyly of the node $X V$ group.

\section{Node XVI Group}

The members of the node XVI group are associated in an unresolved trichotomy comprising Cirripectes polyzona, $\underline{C}$. castaneus, and $\underline{\text { C. }}$ stigmaticus. The node XVI group shares in lacking a pore position behind the ventral edge of the row of nuchal cirri (Figure 17). Two pore positions are present in Exallias, Ophioblennius, and Scartichthys. I interpret the loss of the pore position as a synapomorphy of this group. 


\section{Node XVII Group}

The node XVII group comprises Cirripectes filamentosus, $\underline{\text { C. }}$

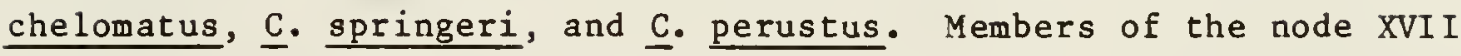
group have a smaller diameter foramen in the infrapharyngobranchial plate (Figure 5) than other members of the node $V$ group. I interpret this decrease in size of the foramen in the infrapharyngobranchial plate as a secondary modification and, as such, a synapomorphy of the node XVII group.

Within the node XVII group, a sister group relationship between

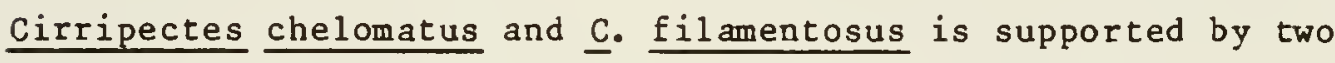
apomorphic characters: (1) adult male genital papilla with two short, closely appressed, slender filaments, with one of the filaments located on either side of the gonopore (Figure 13) and (2) dorsalmost nuchal cirri arising directly from the skin and not connected basally by a low membrane (Figure 7 ).

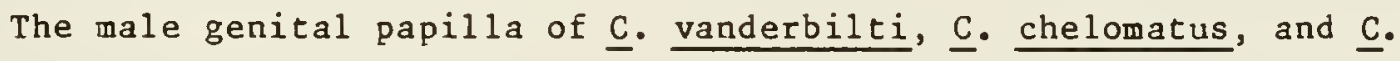
filamentosus has been secondarily modified so that the filaments are shorter and more closely appressed than are the two long, widely separated, slender filaments in other members of the node $\mathrm{X}$ group. I consider the closely appressed filaments in $\underline{C}$. vanderbilti to be an independent modification because this species does not share the characters defining the node XIII and XIV groups. The presence of twu short, closely appressed, slender filaments on the adult male genital papilla is interpreted as a synapomorphy of $\underline{\mathrm{C}}$. $\underline{\text { chelomatus }}$ and $\underline{\mathrm{C}}$. 
As discussed in the account of the node XV group, the dorsalmost nuchal cirri arising directly from the skin is an apomorphic condition, while the dorsalmost cirri connected basally by a low membrane is plesiomorphic. The nuchal cirri morphology in the node XV group is unique among blennioids and appears to have evolved separately from the independently based nuchal cirri in $\underline{C}$. chelomatus and $\underline{C}$. filamentosus. For this reason, I consider the independently based nuchal cirri in $\underline{C}$. chelomatus and $\underline{C}$. Eilamentosus as evidence of monophyly for these two species.

Other relationships within the node XVII group are unresolved. The three possible phylogenies for the node XVII group are shown in Figure 18. Three characters are involved in the different placements of taxa in the cladograms in Figure 18: (1) loss of the mid-snout sensory pore positions (Figure 17),.(2) incomplete lateral line, and (3) a dorsal-fin pterygiophore formula (representing the number of proximal pterygiophores inserting into each of the first four interneural spaces with the first value being the number of proximal pterygiophores inserting anterior to the first neural arch) of $1-1-0-1$.

Cirripectes filamentosus, $\underline{C}$. chelomatus, and $\underline{C}$. springeri lack mid-snout pore positions. All other members of the node III group have one or more pores in this position. The absence of mid-snout pores is a synapomorphy of these three species.

An incomplete lateral line that extends posteriorly to about the middle of the body is shared by Cirripectes filamentosus, $\underline{\text { C. }}$ chelomatus, and $\underline{\mathrm{C}}$. perustus. All other members of the node IV group 


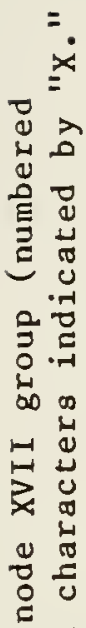

()

들

.

崩

赵

ह

4

क न

를

प्र

$\infty$

ว

로오

อ

.

的

a

굴

$-10$

ฮั

$\rightarrow \infty$

$\rightarrow \rightarrow$

뭉

(4) 7

(1)

点

$\infty$ क

$\infty$

(1)

范

. $\rightarrow$

a

焉 

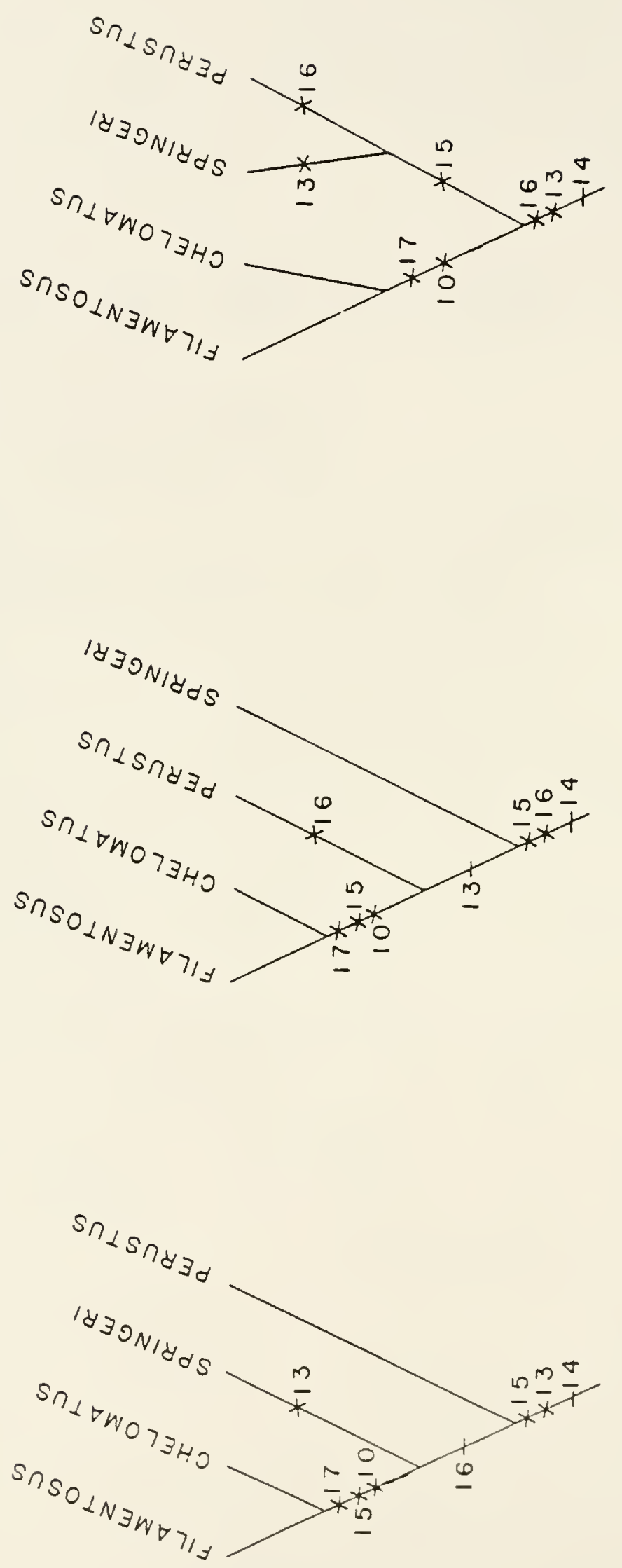
have a complete lateral liac (reaches at least to caudal peduncle). An incomplete lateral line is a synapomorphy of these three species.

A dorsal-fin pterygiophore formula of $1-1-0-1$ is shared only by Cirripectes stigmaticus, ‥ perustus, ‥ springeri, and Ophioblennius. The dorsal-fin pterygiophore formula is typically $0-2-0-1$ in the Salariini. Although the dorsal-fin pterygiophore formula varies among blennioid fishes, I have not found the 1-1-0-1 formula in other blennioids. The 1-1-0-1 formula is apomorphic, but occurs homoplasiously and, based on a parsimony argument, has been derived independencly in Ophioblennius and twice within Cirripectes (in the node XVI and XVII groups).

Because the mid-snout pore, lateral-line, and dorsal-fin pterygiophore formula characters each support a different pattern of phylogenetic relationships, the relationships among Cirripectes springeri, $\underline{C}$. perustus, and the $\underline{C}$. filamentosus $-\underline{C}$. chelomatus group must remain unresolved until one or more additional characters are discovered that support one of the three hypotheses of relationship. 


\section{Cirripectes SWAINSON}

Cirripectes Swainson, 1839:182, 275 (type species Salarias variolosus Valenciennes in Cuvier and Valenciennes, 1836, by original designation). Spelled Cirripectus on pp. 79, 80.

Diagnosis. Cirripectes is a salariin blenniid with: (1) a transverse row of uniform-length, simple, nuchal cirri that may be in a coiiinuous row or in up to four groups, but, when in more than one group, with little or no gap at the dorsalmost point on the nape; and (2) an adult male urogenital papilla with one or two tapering filaments extending beyond the gonopore. Among blenniids, these conditions are unique to Cirripectes.

Cirripectes may also be distinguished from other salariin genera with a transverse row of nuchal cirri by the following combination of characters: no vomerine teeth, large canine located posteriorly on each dentary bone, 13-17 (rarely 13) segmented dorsal-fin rays, and 14-18 segmented anal-fin rays. Selected counts for the species of Cirripectes are provided in Table 3.

Distribution. Cirripectes is widely distributed throughout the Indo-Pacific region (Figures 19-26).

Nomenclatural discussion. Swainson (1839) used two spellings in the publication describing this genus. Early in his paper, he 


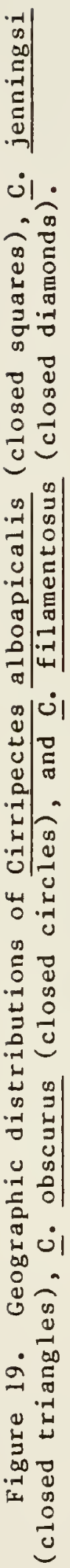




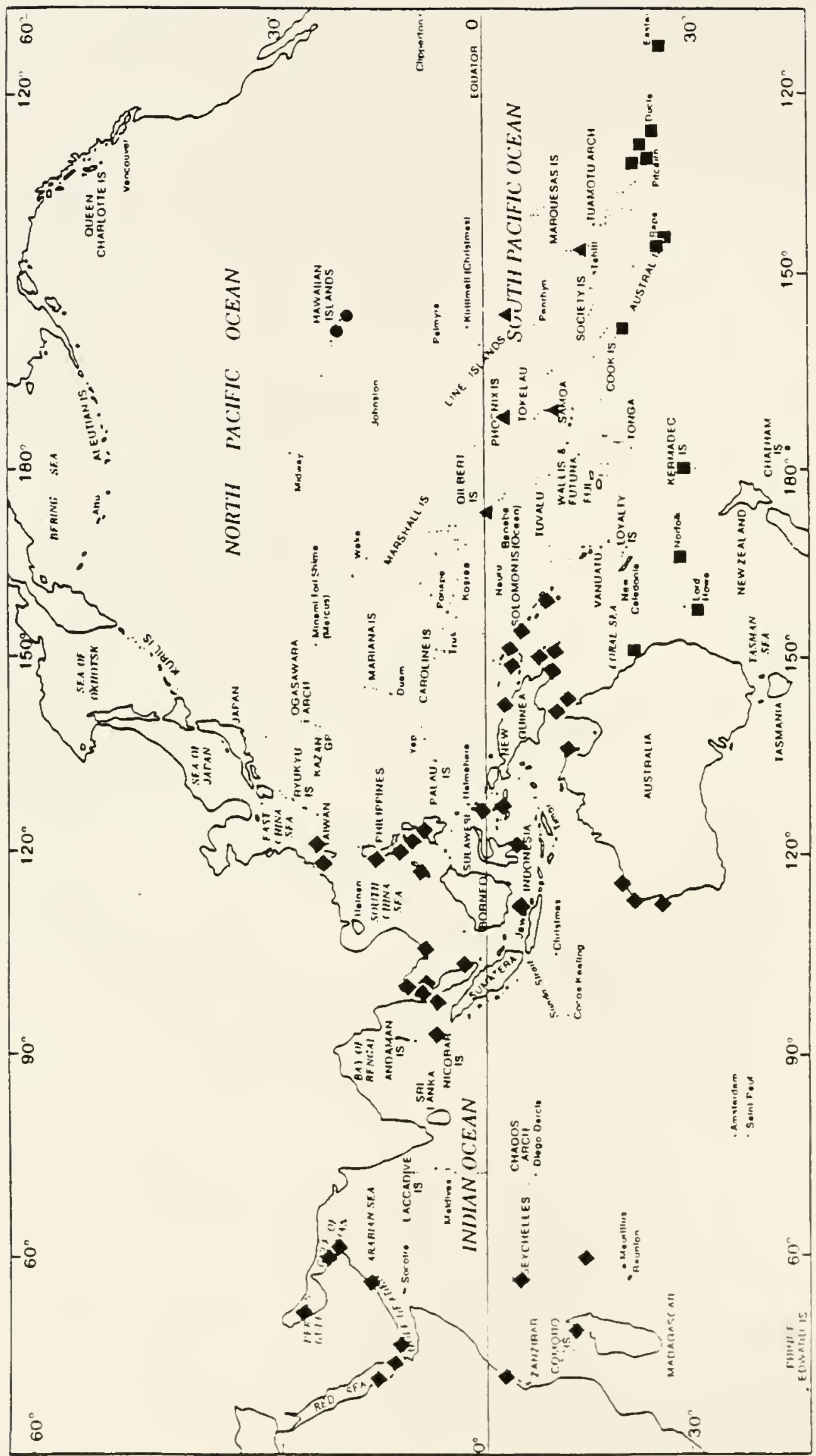




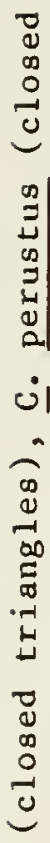

先|

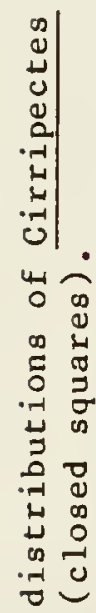

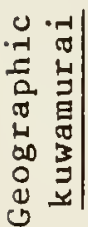

ن

류

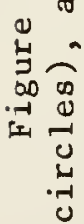




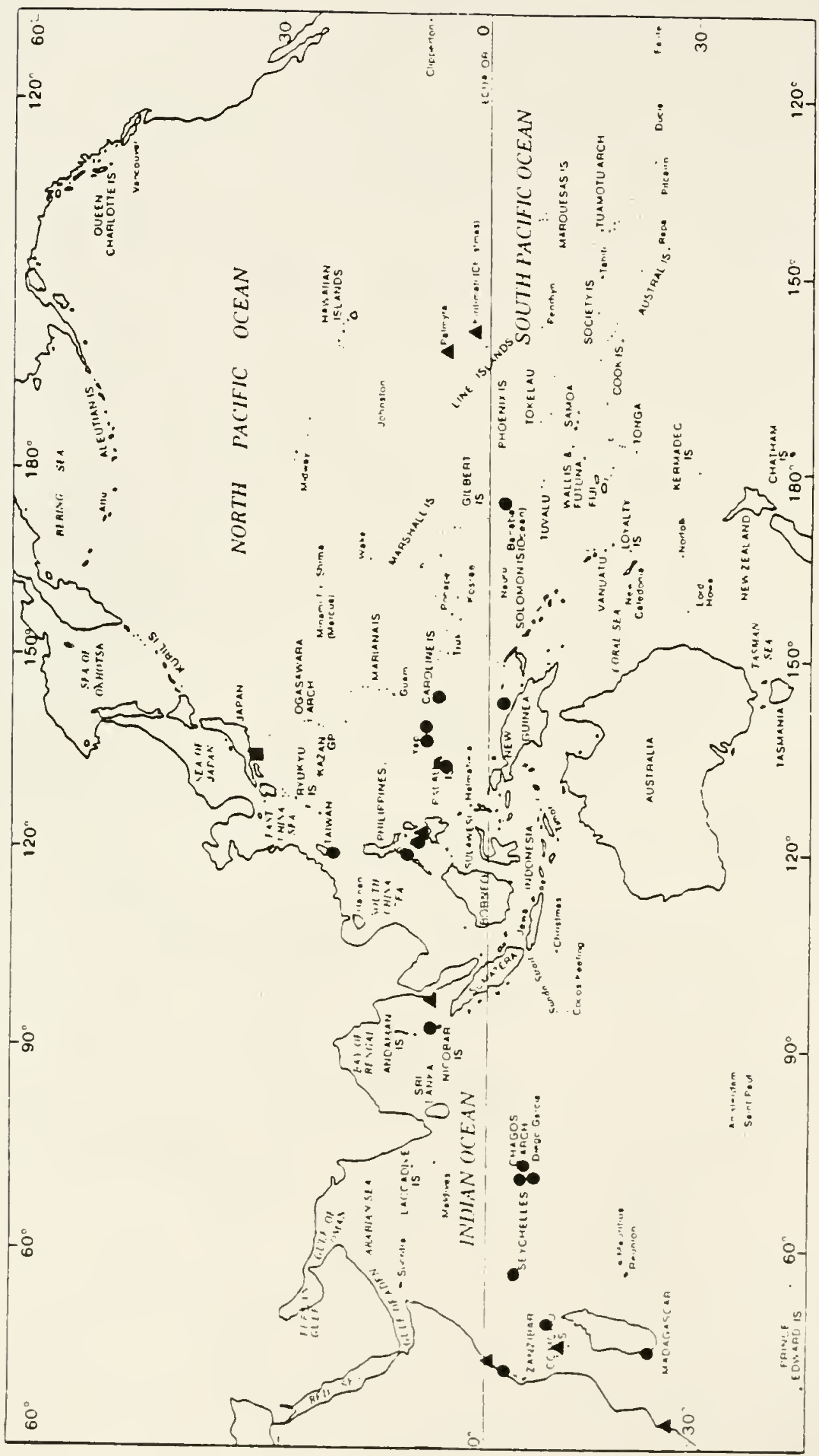




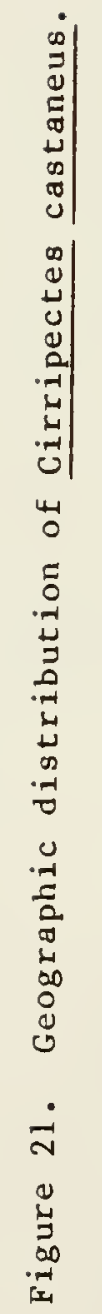




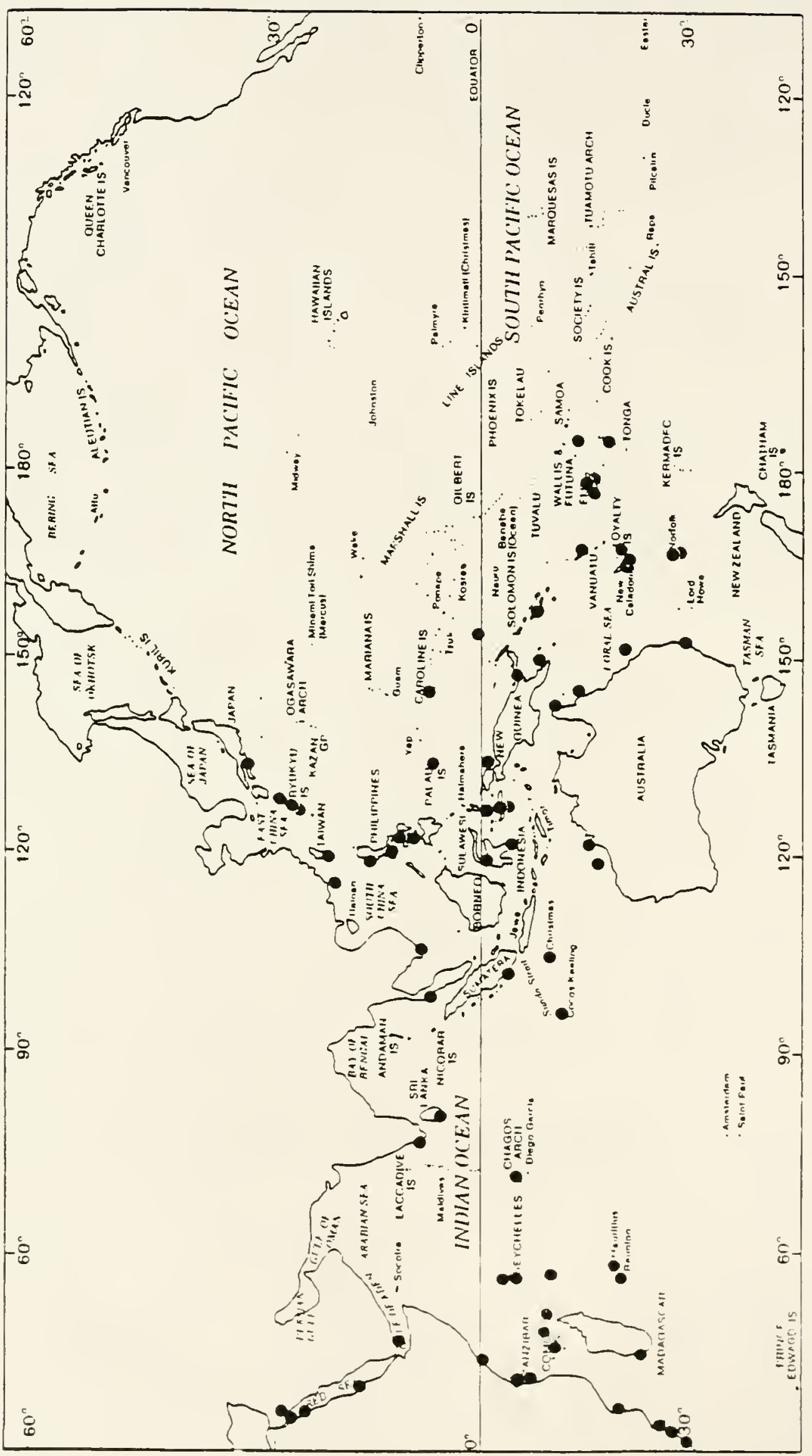




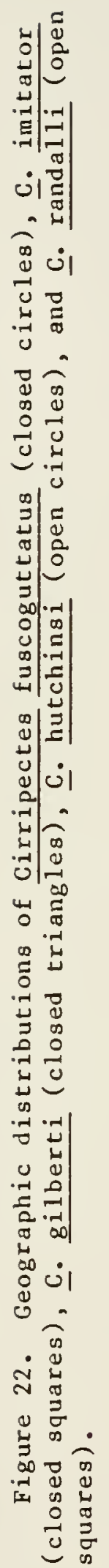




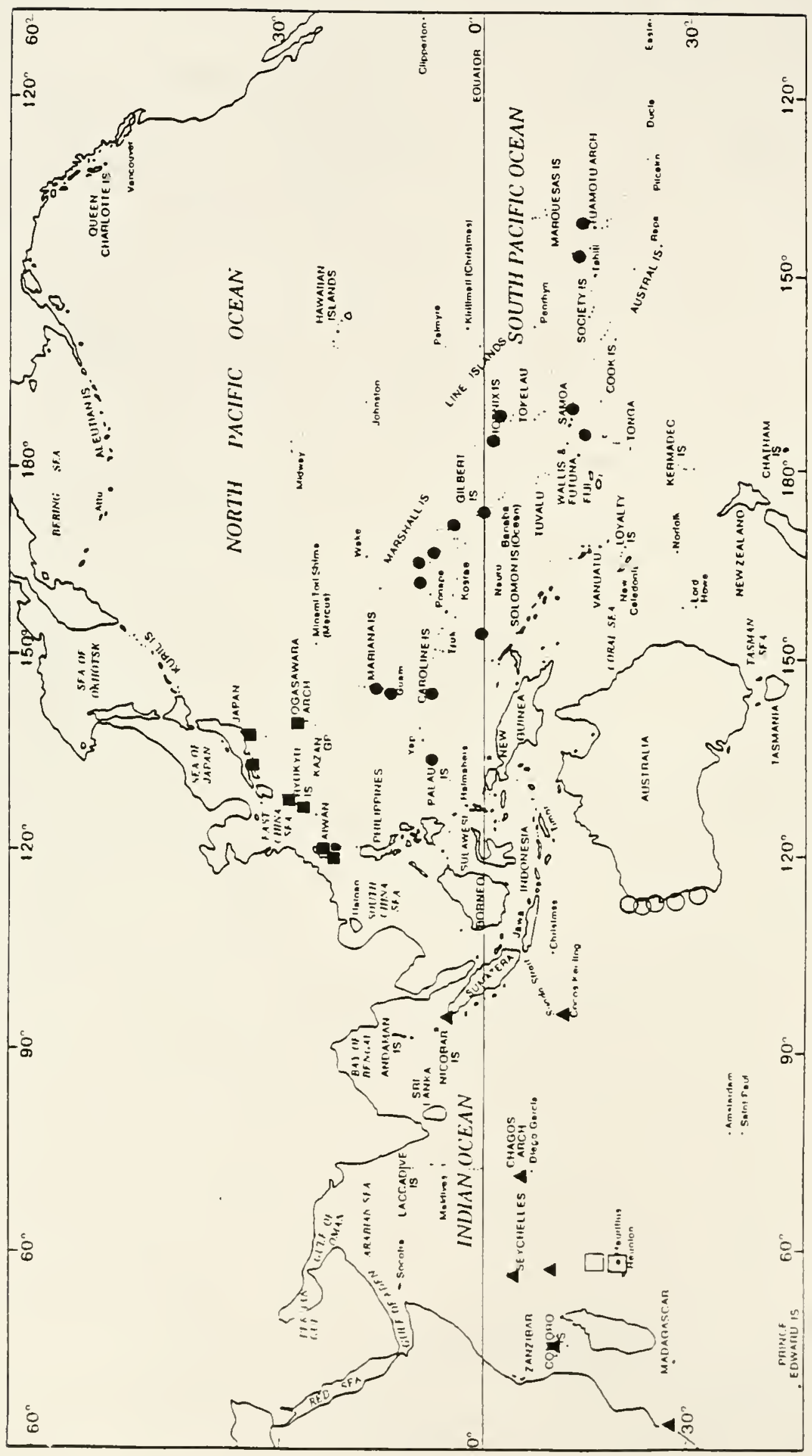




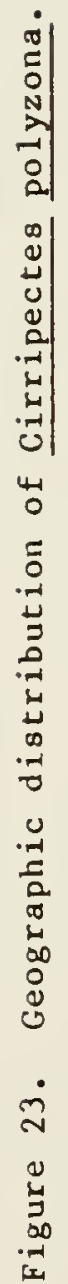




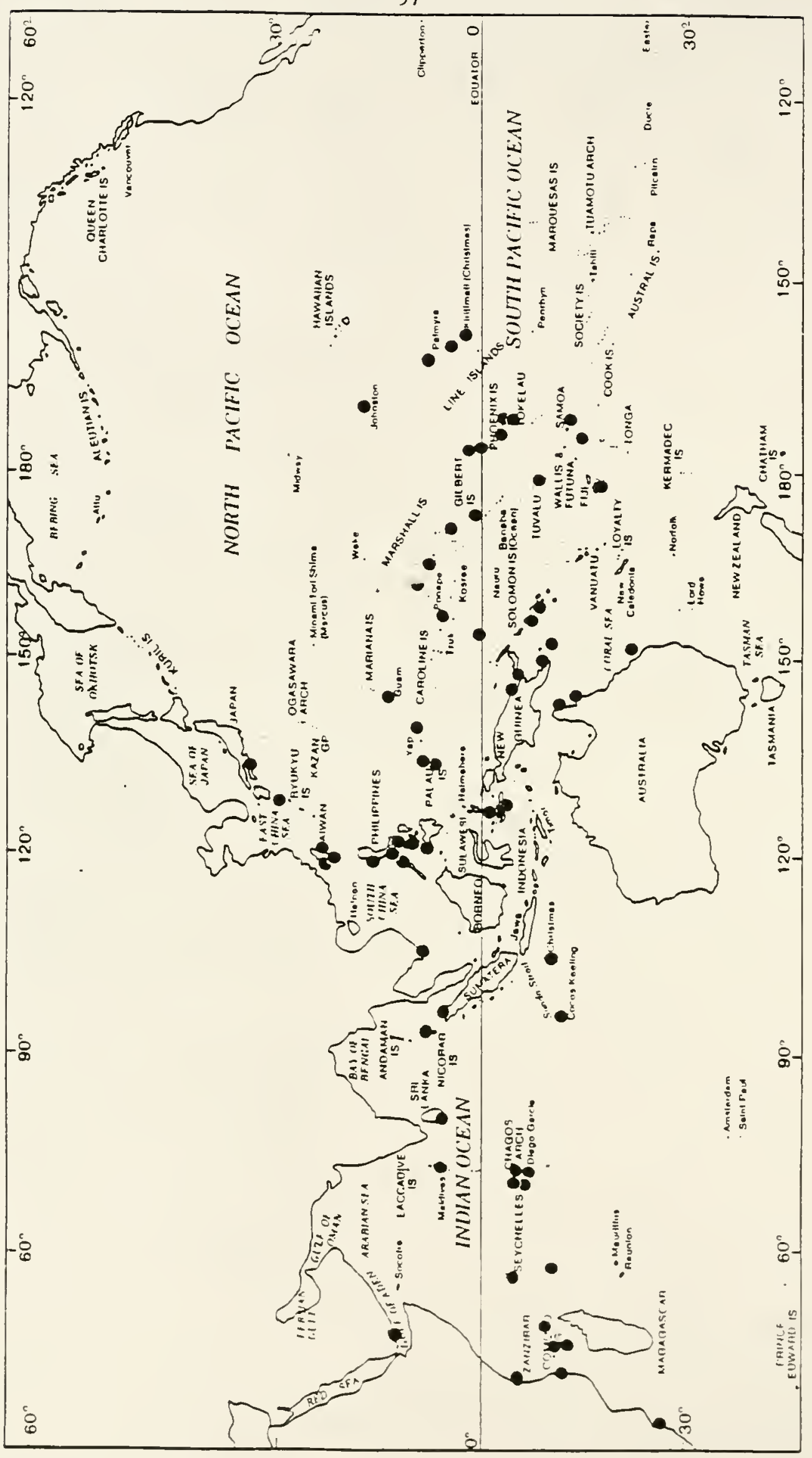





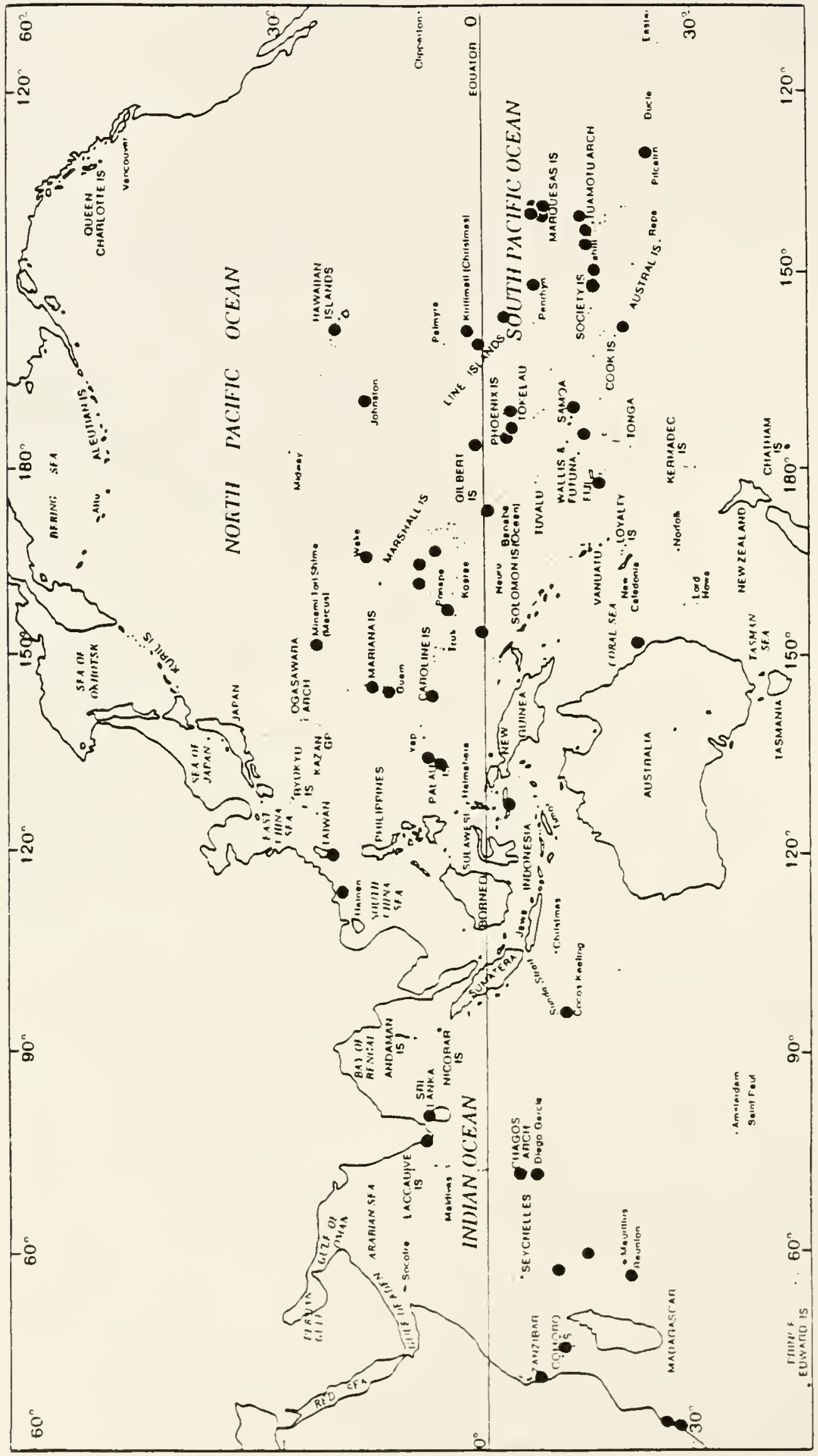




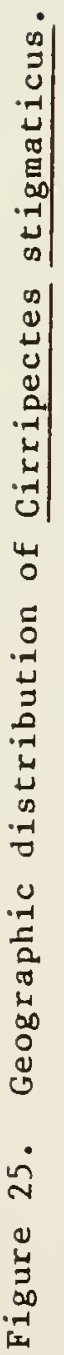




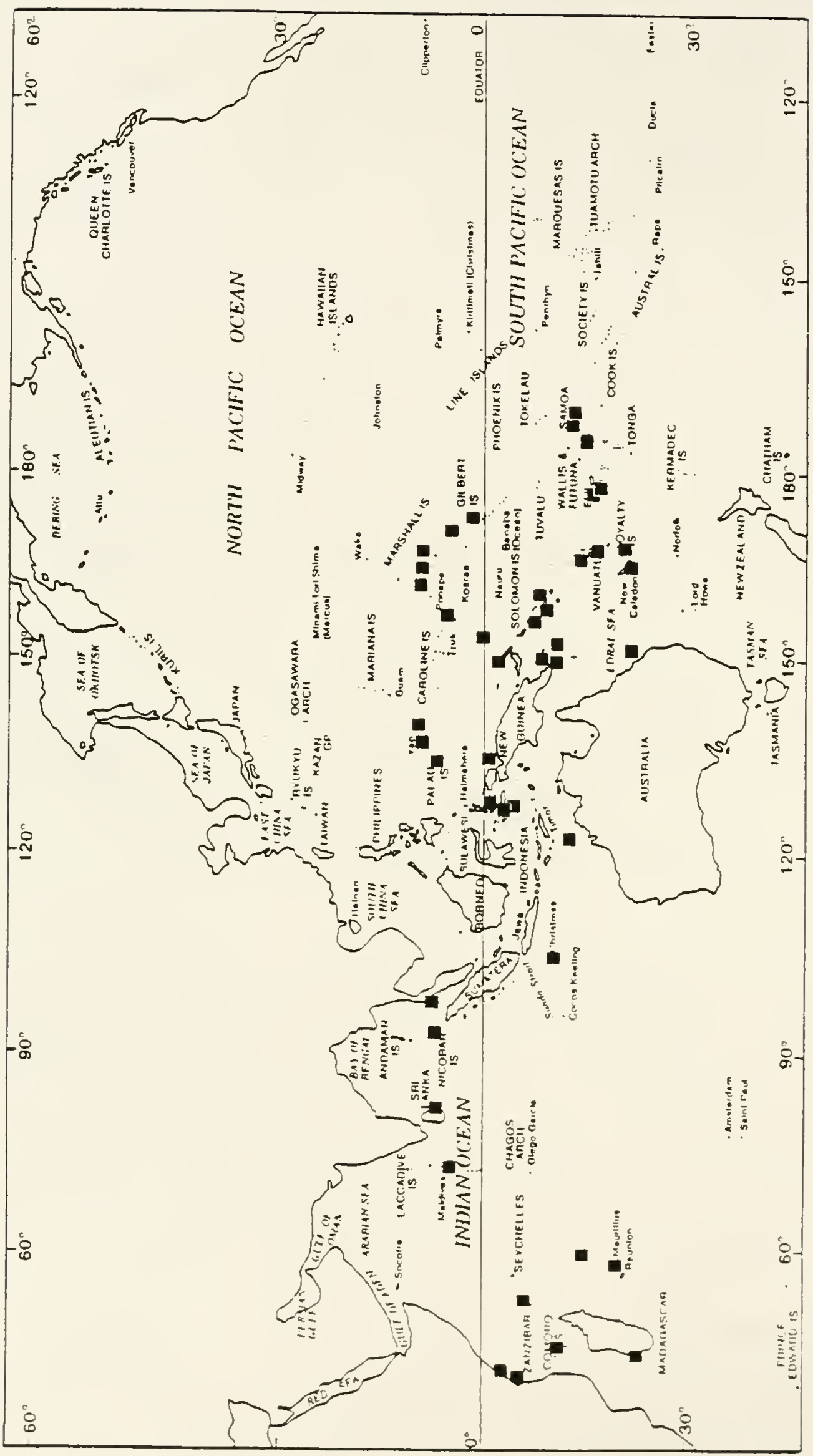




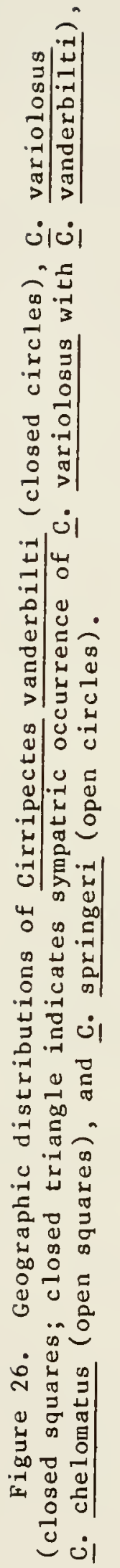




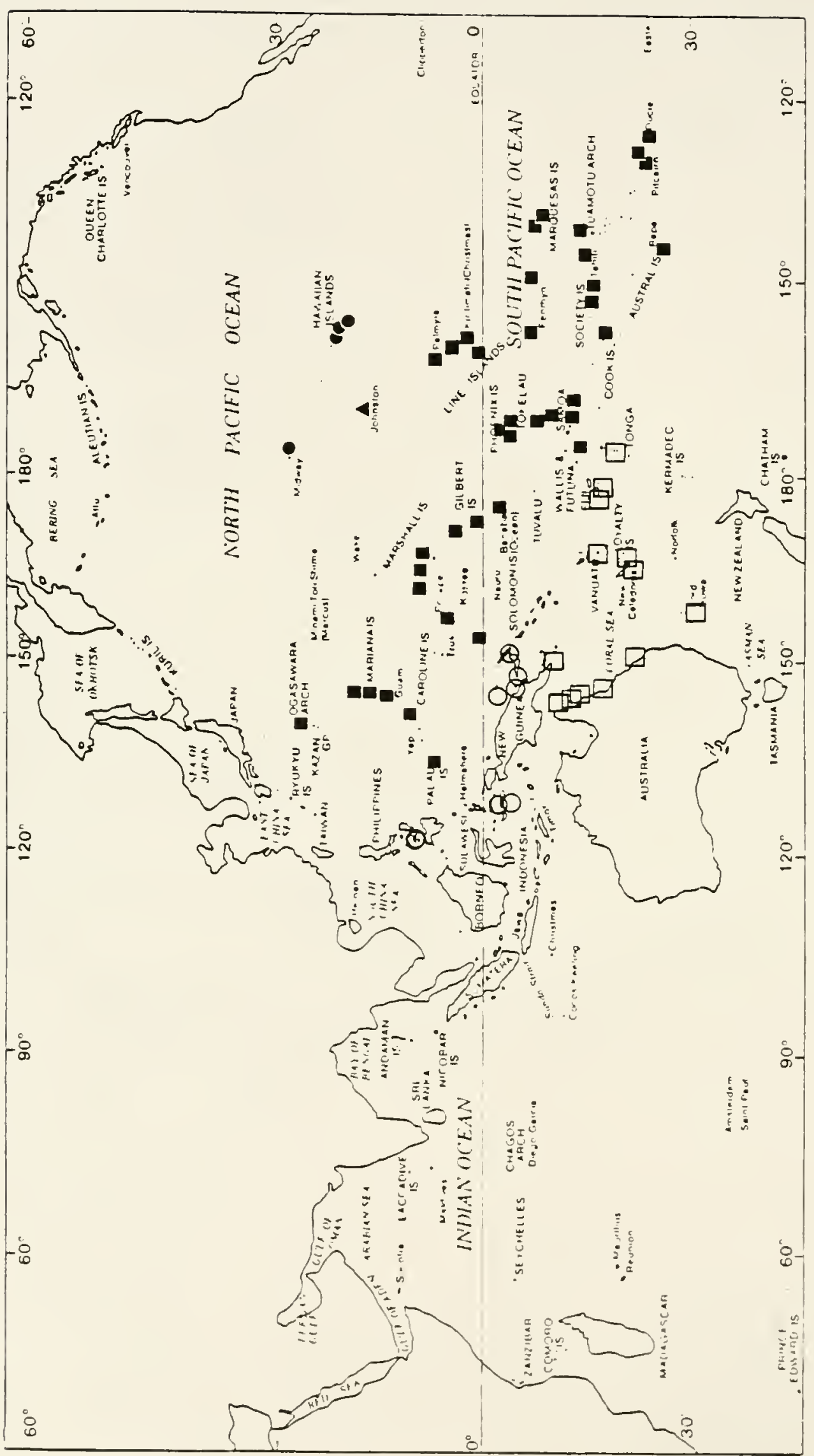


Table 3. Counts for selected characters of the species of Cirripectes. Table headings: 1 st - primary modal count, 2nd secondary modal count for bimodal characters, $n$ - number of specimens.

\begin{tabular}{|c|c|c|c|c|c|c|c|c|}
\hline & \multicolumn{4}{|c|}{ Segmented Dorsal Fin Rays } & \multicolumn{3}{|c|}{ Segmented Anal Fin } & \multirow{2}{*}{$\begin{array}{r}\text { Rays } \\
\mathrm{n} \\
\end{array}$} \\
\hline & Range & $\underline{1 s t}$ & $\underline{2 n d}$ & $\mathrm{n}$ & Range & $1 \mathrm{st}$ & ?nd & \\
\hline alboapicalis & $15-17$ & 16 & & 119 & $16-18$ & 17 & & 113 \\
\hline auritus & $15-17$ & 16 & & 13 & $16-18$ & 17 & & 13 \\
\hline castaneus & $13-15$ & 14 & & 861 & $14-16$ & 15 & & 852 \\
\hline chelomatus & $14-16$ & 15 & & 143 & $15-17$ & 16 & & 139 \\
\hline Eilamentosus & $13-16$ & 15 & 14 & 574 & $14-17$ & 16 & 15 & 554 \\
\hline Euscoguttatus & $13-15$ & 14 & & 140 & $14-16$ & 15 & & 140 \\
\hline gilberti & 14 & & & 22 & 15 & & & 23 \\
\hline hutchinsi & $13-14$ & 14 & & 19 & $15-16$ & 15 & & 19 \\
\hline imitator & $13-15$ & 14 & & 63 & $14-16$ & 15 & & 63 \\
\hline jenningsi & $15-16$ & 15 & & 94. & $15-17$ & 16 & & 94 \\
\hline kuwamurai & 16 & & & 1 & 17 & & & 1 \\
\hline obscurus & $15-17$ & 16 & & 50 & $16-17$ & 17 & & 47 \\
\hline perustus & $14-15$ & 14 & & 78 & $14-16$ & 15 & & 78 \\
\hline polyzona & $13-15$ & 14 & & 396 & $14-16$ & 15 & & 395 \\
\hline quagga & $14-16$ & 15 & & 441 & $15-17$ & 16 & & 440 \\
\hline randalli & $14-15$ & 14 & & 8 & $15-16$ & 15 & & 8 \\
\hline springeri & $14-16$ & 15 & & 46 & $15-17$ & 16 & & 47 \\
\hline stigmaticus & $14-16$ & 15 & & 213 & $15-17$ & 16 & & 211 \\
\hline vanderbilti & $13-15$ & 14 & & 116 & $15-i 6$ & 15 & & 116 \\
\hline yariolosus & $13-15$ & 14 & & 491 & $14-16$ & 15 & & 486 \\
\hline
\end{tabular}


Table 3. Continued.

\begin{tabular}{|c|c|c|c|c|c|c|}
\hline & \multicolumn{3}{|c|}{ Caudal Vertebrae } & \multicolumn{3}{|c|}{ Totai Vertebrae } \\
\hline & Range & $1 \mathrm{st}$ & $\mathrm{n}$ & Range & $1 \mathrm{st}$ & $\mathrm{n}$ \\
\hline alboapicalis & $21-23$ & 22 & 115 & $31-33$ & 32 & 115 \\
\hline auritus & $21-23$ & 22 & 13 & $31-33$ & 32 & 13 \\
\hline castaneus & $19-21$ & 20 & 846 & $29-31$ & 30 & 839 \\
\hline chelomatus & $19-21$ & 20 & 136 & $29-31$ & 30 & 112 \\
\hline filamentosus & $19-21$ & 20 & 564 & $29-31$ & 30 & 564 \\
\hline Euscoguttatus & 20 & & 135 & 30 & & 135 \\
\hline gilberti & 20 & & 23 & 30 & & 23 \\
\hline hutchinsi & $20-21$ & 20 & 19 & $30-31$ & 30 & 19 \\
\hline imitator & 20 & & 63 & 30 & & 63 \\
\hline jenningsi & $21-22$ & 21 & 94 & $31-32$ & 31 & $9 \ddot{4}$ \\
\hline kuwamurai & 22 & & 1 & 32 & & 1 \\
\hline obscurus & $21-23$ & 22 & 47 & $31-33$ & 32 & 47 \\
\hline perustus & $20-21$ & 20 & 78 & $30-31$ & 30 & 74 \\
\hline polyzona & $19-21$ & 20 & 396 & $29-31$ & 30 & 396 \\
\hline quagga & $20-23$ & 21 & 446 & $30-32$ & 31 & 446 \\
\hline randalli & 20 & & 8 & 30 & & 8 \\
\hline springeri & $20-21$ & 21 & 45 & $30-31$ & 31 & 45 \\
\hline stigmaticus & $20-22$ & 21 & 203 & $30-32$ & 31 & 203 \\
\hline vanderbilti & $20-21$ & 20 & 114 & $3 \hat{y}-31$ & 30 & 114 \\
\hline variolosus & $19-21$ & 20 & 483 & $29-31$ & 30 & 483 \\
\hline
\end{tabular}


Table 3. Continued.

Last Epipleural Rib on

$\frac{\text { Vertebral centrum }}{\text { Range 1st 2nd } n}$

Left+Right Orbital Cirri

Range lst 2nd n

$\begin{array}{lllll}\text { alboapicalis } & 18-23 & 20 & 21 & 113\end{array}$

$\begin{array}{llll}4-18 & 8 & 6 & 83\end{array}$

auritus

$\begin{array}{llll}17-19 & 18 & 19 & 8\end{array}$

$2-$

$\begin{array}{lll}6 & 4 & 13\end{array}$

castaneus

$\begin{array}{llll}17-23 & 19 & 18 & 840\end{array}$

$6-49 \quad 10 \quad 11 \quad 200$

$\begin{array}{lllll}\text { chelomatus } & 15-18 & 16 & 17 & 133\end{array}$

$\begin{array}{llll}3-93 & 8 & 6 & 82\end{array}$

$\begin{array}{lllll}\text { filamentosus } & 13-17 & 15 & 14 & 557\end{array}$

$\begin{array}{llll}4-13 & 6 & 8 & 147\end{array}$

$\begin{array}{lllll}\text { fuscoguttatus } & 17-20 & 19 & 18 & 135\end{array}$

$\begin{array}{llll}18-42 & 32 & 29 & 48\end{array}$

gilberti

$\begin{array}{llll}17-19 & 17 & 18 & 23\end{array}$

$\begin{array}{llll}17-48 & 27 & 22 & 24\end{array}$

hutchinsi

$16-20 \quad 18$

19

$12-29 \quad 20$

$19 \quad 19$

imitator

$\begin{array}{llll}18-21 & 19 & 20 & 62\end{array}$

$13-46$

$19 \quad 26$

52

jenningsi

$20-23$

22

$21 \quad 94$

$2-4$

85

kuwamurai

19

12

1

obscurus

$\begin{array}{llll}19-22 & 20 & 21 & 47\end{array}$

$10-26 \quad 16$

24

perustus

$15-19$

78

$6-23 \quad 14$

17

63

polyzona

15-19 17

389

4-15 $\quad 10$

1178

quagga

19-24

21

$22 \quad 451$

$2-15 \quad 6$

2

166

randalli

$18-20 \quad 19$

8

i $2-41$

8

$\begin{array}{llll}\text { springeri } & 16-19 & 17 & 44\end{array}$

2-9

6

46

$\begin{array}{lllll}\text { stigmaticus } & 17-21 & 19 & 18 & 207\end{array}$

$7-22 \quad 12$

43

$\begin{array}{lllll}\text { vanderbilti } & 18-21 \quad \text { ig } & 20 & 115\end{array}$

$7-22 \quad 14$

95

variolosus

$18-23 \quad 20$

$21 \quad 470$

4-14

6

$8 \quad 70$ 
Table 3. Continued.

Left+Right Nasal Cirli

Range 1st 2nd $\mathrm{n}$

Total Nuchal Cirri Bases

Range 1st 2nd n

\begin{tabular}{|c|c|c|c|c|c|c|c|c|}
\hline alboapicalis & $8-27$ & 10 & 11 & 84 & $28-42$ & 35 & & 112 \\
\hline auritus & $4-6$ & 6 & & 12 & $22-34$ & 34 & & 7 \\
\hline castaneus & $6-49$ & 10 & 8 & 222 & $29-44$ & 36 & 37 & 215 \\
\hline chelomatus & $6-82$ & 10 & & 85 & $22-45$ & 28 & 29 & 67 \\
\hline filamentosus & $4-34$ & 8 & & 148 & $22-39$ & 29 & 26 & 149 \\
\hline fuscoguttatus & $7-32$ & 22 & & 44 & $47-66$ & 52 & 55 & 47 \\
\hline gilberti & $11-33$ & 12 & & 24 & $50-64$ & 59 & 56 & 23 \\
\hline hutchinsi & $8-31$ & 17 & & 19 & $35-42$ & 38 & & 19 \\
\hline imitator & $9-93$ & 11 & 12 & 52 & $40-59$ & 49 & 51 & 51 \\
\hline jenningsi & $8-19$ & 10 & 11 & 70 & $34-44$ & 42 & 40 & 71 \\
\hline kuwamurai & 10 & & & 1 & 31 & & & 1 \\
\hline obscurus & $14-42$ & 21 & & 25 & $36-47$ & 40 & & 44 \\
\hline perustus & $5-25$ & 10 & & 63 & $30-46$ & 39 & 40 & 64 \\
\hline polyzona & $5-24$ & 10 & & 76 & $32-44$ & 39 & 38 & 76 \\
\hline quagga & $6-16$ & 10 & 11 & 158 & $23-36$ & 28 & 30 & 160 \\
\hline randalli & $10-18$ & 17 & & 7 & $36-42$ & 40 & 41 & 8 \\
\hline springeri & $5-23$ & ó & 7 & 47 & $27-38$ & 31 & & 45 \\
\hline$\underline{\text { stigmaticus }}$ & $8-29$ & 12 & $1:$ & 43 & $34-47$ & 37 & & 47 \\
\hline vanderbilti & $7-23$ & 9 & 8 & 96 & $31-42$ & 36 & 38 & 94 \\
\hline variolosus & $4-19$ & 8 & 10 & 70 & $27-36$ & 31 & & 68 \\
\hline
\end{tabular}


Table 3. Continued.

Last LI Tube Beneath
Dorsal Fin Element ${ }^{a}$
Range Ist $\underline{2 n d} \quad n$

Number of LL Tubes in

Adults (over $35 \mathrm{~mm}$ SL)

Range 1st 2nd n

alboapicalis 12-end end

53

$9-17$

14

13

74

auritus

$5-13$

6

$7-12$

7

castaneus

5-end end

176

$1-13$

8

9

175

chelomatus

$12-15 \quad 5$

4

82

$0-8$

2

3

77

filamentosus

$X I I I$

5

210

$0-8$

2

3

195

fuscoguttatus 13-end end

$0-5$

$0 \quad 1$

gilberti

9-end end

21

$0-5$

1324

hutchinsi

13-end end

17

$2-9$

7

6

imitator

9-end end

58

5-14

10

56

jenningsi

15-end end

46

2-11

8

88

kuwamurai

7

1

6

obscurus

end

42

$0-10$

6

perustus

5-11

59

$0-3$

1

$0 \quad 45$

polyzona

7-end 12

66

2-9

5

$7 \quad 58$

quagga

7-end end

15

115

$7-18$

14

$13 \quad 120$

randa11i

end

8

$1-7$

5

7

$\underline{\text { springeri }}$

$2-8$

42

$0-5$

3

233

$\underline{\text { stigmaticus }}$

7-end 11

1243

$1-10$

5

vanderbilti

$\begin{array}{llll}7 \text {-end } & 12 & 10 & 89\end{array}$

$0-5$

$2 \quad 3 \quad 90$

variolosus

7-end 12

62

2-11

$8 \quad 7 \quad 60$

"end" means the last LL tube is on the base of the caudal fin. 
mentioned the generic name and gave the spelling as Cirripectus, but in the description of the genus on page 275 , he used the spelling Cirripectes. Schuliz and Chapman in Schultz et al. (1960) discussed the problem of the valid spelling of this generic name. They stated correctly that the first subsequent user of the rame was Bleeker (1868), who used it as a subgenus in his description of Salarias (Cirripectes) polyzona, and that the first revisers were McCulloch and McNeil (1918), who selected Cirripectes as the correct spelling and treated Cirripectus as a synonym. Subsequent studies of the genus Cirripectes using the spelling Cirripectus (eg. Normar., 1943; Smith, 1959; Strasburg and Schultz, 1953) are not first revisions and, thus, can not be used to establish the spelling of the generic name.

\section{Key to the Species of Cirripectes}

1a. Large fleshy flap bearing small nuchal cirri on either side of nape (Figure 7A); sevcr? 1 independently based cirri across nape between

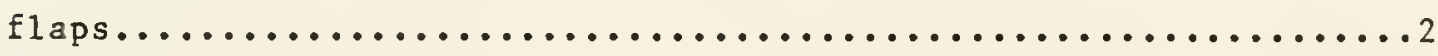

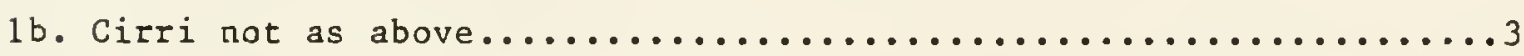

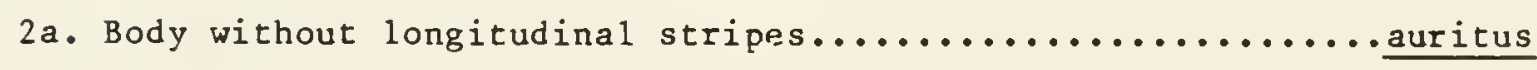

(Figure 29)

2b. Body with five pale (red in life) longitudinal stripes...kuwamurai 
3a. Dorsal fin XII, 15 or 16; anal fin II, 16 or 17 (some specimens of

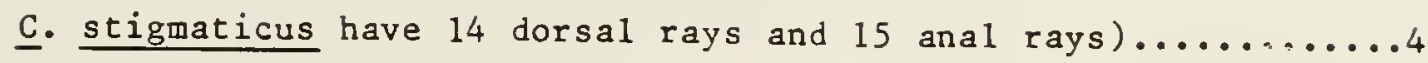

3b. Dorsal fin $X I I, 14$; anal fin $I I, 15 \ldots \ldots \ldots \ldots \ldots \ldots \ldots \ldots \ldots \ldots$

4a. Lower lip crenulate medially; cephalic sensory pore system with 6 or more pores at each preopercular (POP) position (especially upper positions.................................

4b. Lower lip entire medially; each POP position with 1 or 2 pores, occasionally 4 or 5 at upper positions..................

5a. Lateral line with scalelike flaps anteriorly; segmented dorsal-fin rays usually 16, anal rays usually 17; pupil-sized black spot behind eye; first dorsal-fin spine not elongate in adults. .6

5b. No laterá! line flaps; dorsal-fin rays usually 15; anal rays usually 16; no dark spot behind eye; first dorsal-fin spine elongate in adult males............................

6a. Typically fewer than 9 distinct laceral line tubes; Hawaiian

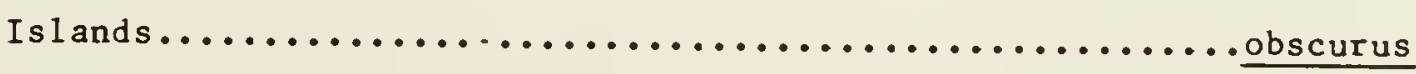


6b. More than 8 lateral line tubes; South Pacific south of $20^{\circ} \mathrm{S}$ latitude alboapicalis

(Figures 27, 28)

7a. Dorsal-fin membrane attached to caudal fin in adults; no gap in row of nuchal cirri on nape................... jenningsi (Figure 45)

7b. Dorsal-fin membrane not attached to caudal fin in adults; row of nuchal cirri with a distinct gap ou nape.............. quagga

(Figures 53-55)

8a. Dorsal fin entire; posterior half to three-quarters of body with small brown spots (dark red in life) in both sexes......chelomatus

(Figure 33)

8b. Dorsal fin distinctly notched; color not as above............9

9a. Last lateral-1ine tube beneath or posterior to vertical at base of

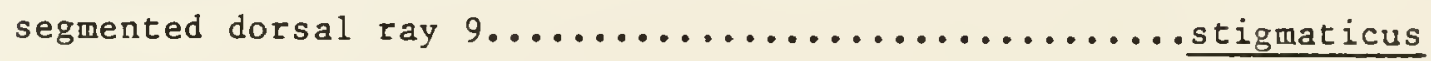

9b. Last lateral-line tube anterior to vertical at base of segmented

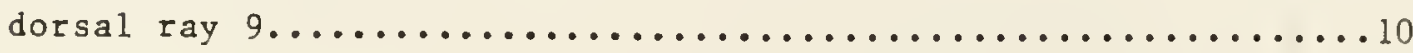


10a. Body uniformly brown and head with pale diagonally directed bars or small pale spots; caudal vertebrae $20 \ldots$... filamentosus (in part)

(Figure 34)

10b. Small dark-brown spots on body and small pale (red in life) spots on head; caudal vertebrae $21 \ldots \ldots \ldots \ldots \ldots \ldots \ldots \ldots \ldots$........................

(Figure 58)

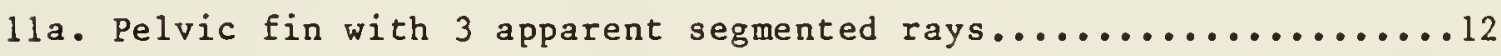

11b. Pelvic fin with 4 apparent segmented rays (inner ray small and sometimes difficult to discern) $\ldots \ldots \ldots \ldots \ldots \ldots \ldots \ldots \ldots \ldots \ldots \ldots \ldots$

12a. Posteriormost lateral-line tube at or posterior to vertical from base of segmented dorsal-fin ray 7; POP positions with 6 or more pores at most positions; small pale spots on throat; body with alternating dark and pale bars.................... polyzona

12b. Posteriormost lateral-line tube not reaching beyond vertical from segmented dorsal-fin ray 7; most POP positions with 1 or 2 pores;

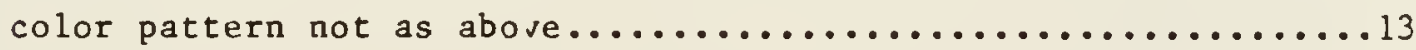


13a. Dorsal fin notched; in alcohol, males and females with uniformly brown body and small pale spots on head; male genital filaments closely appressed (Figure 13$) \ldots \ldots \ldots \ldots$ filamentosus (in part)

(Figure 34)

13b. Dorsal fin entire; in alcohol, males with head and body uniformly brown (in life, anterior half of body yellowish brown and posterior half of body reddish brown); females with small dark-brown spots on brown body; male genital filaments widely separated (Figure 13$) \ldots \ldots \ldots \ldots \ldots \ldots \ldots \ldots \ldots$ perustus (Figures 49, 50)

14a. Cephalic sensory pore system with 6 or more pores at each POP position... 16

14b. Each POP position with I or 2 pores, occasionally 4 or 5 at upper positions in large specimens.........................

15a. Snout and cheeks with very small, pale spots or bars (red in life); body uniform brown; last lateral-line tube lies at or before vertical from base of dorsal-fin ray $6 \ldots \ldots \ldots$ filamentosus

(in part; Figure 34)

15b. Color not as above; last lateral-line tube lies at or posterior to vertical from dorsal-fin ray $6 \ldots \ldots \ldots \ldots \ldots \ldots \ldots \ldots$ castaneus (Figures 30-32; some stigmaticus may key to this couplet) 
16a. Nuchal cirri arranged in four groups with lateralmost cirri borne on a small-to-large fleshy flap (Figure $7 \mathrm{C}$ )................

16b. Nuchal cirri arranged in two groups with a small gap at the nape (Figure 7E); Hawaiian Islands and Johnston Island endemic

vanderbilti

(Figure 61)

17a. Lateralmost nuchal cirri borne on a greatly expanded fleshy flap (Figure $7 \mathrm{C}$ ); 43 or more distinct nuchal cirri bases..........18

17b. Lateralmost nuchal cirri borne on a small fleshy flap (Figure 7D); 42 or fewer distinct nuchal cirri bases................... 20

18a. Number of lateral line tubes 7 to 14; Taiwan to southern Japan and the ogasawara Islands........................ imitator (Figures 43, 44)

18b. Number of lateral line tubes 0 to $5 \ldots \ldots \ldots \ldots \ldots \ldots \ldots \ldots$

19a. Color pattern of head and body of males and females consisting of dark browr spots on a pale brown background; widely distributed on Pacific Plate as far west as Taiwan............... fuscoguttatus 
19b. Males with uniform brown head and body; females with pale (maroon in life) spots on head and body; restricted to Indian Ocean gilberti

(Figures 37-39)

20a. Total number of orbital cirri free tips on both sides 4 to 13;

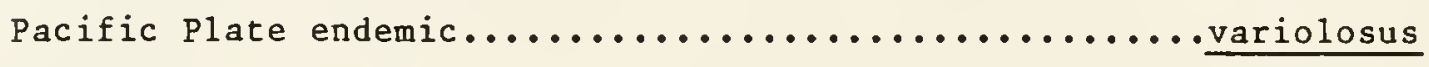
(Figure 63)

20b. Typically more than 13 orbital cirri free tips; Indian ocean....21

21a. Color pattern of head and body of males and females with small dark brown spots on brown background; Mauritius and Cargados Carajos Shoals................................ randalli

(Figures 56,57 )

21b. Males with five or six broad dark brown bars on body; females similar to males in color pattern but may have small dark brown spots on head and anterior half of body; southwestern

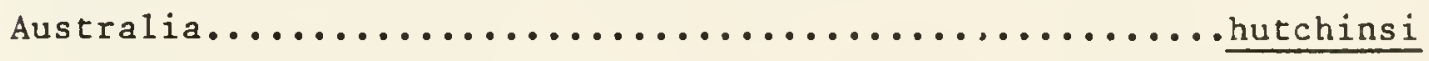


Figure 27. Female Cirripectes alboapicalis, Lord Howe Island (AMS I.17424-008: $66 \mathrm{~mm}$ SL).

Figure 28. Male Cirripectes alboapicalis, Mangaia, Cook Islands (CAS uncat.: $122 \mathrm{~mm}$ SL). mm SL).

Figure 29. Male Cirripectes auritus, South Africa (RUSI 8008: 37 

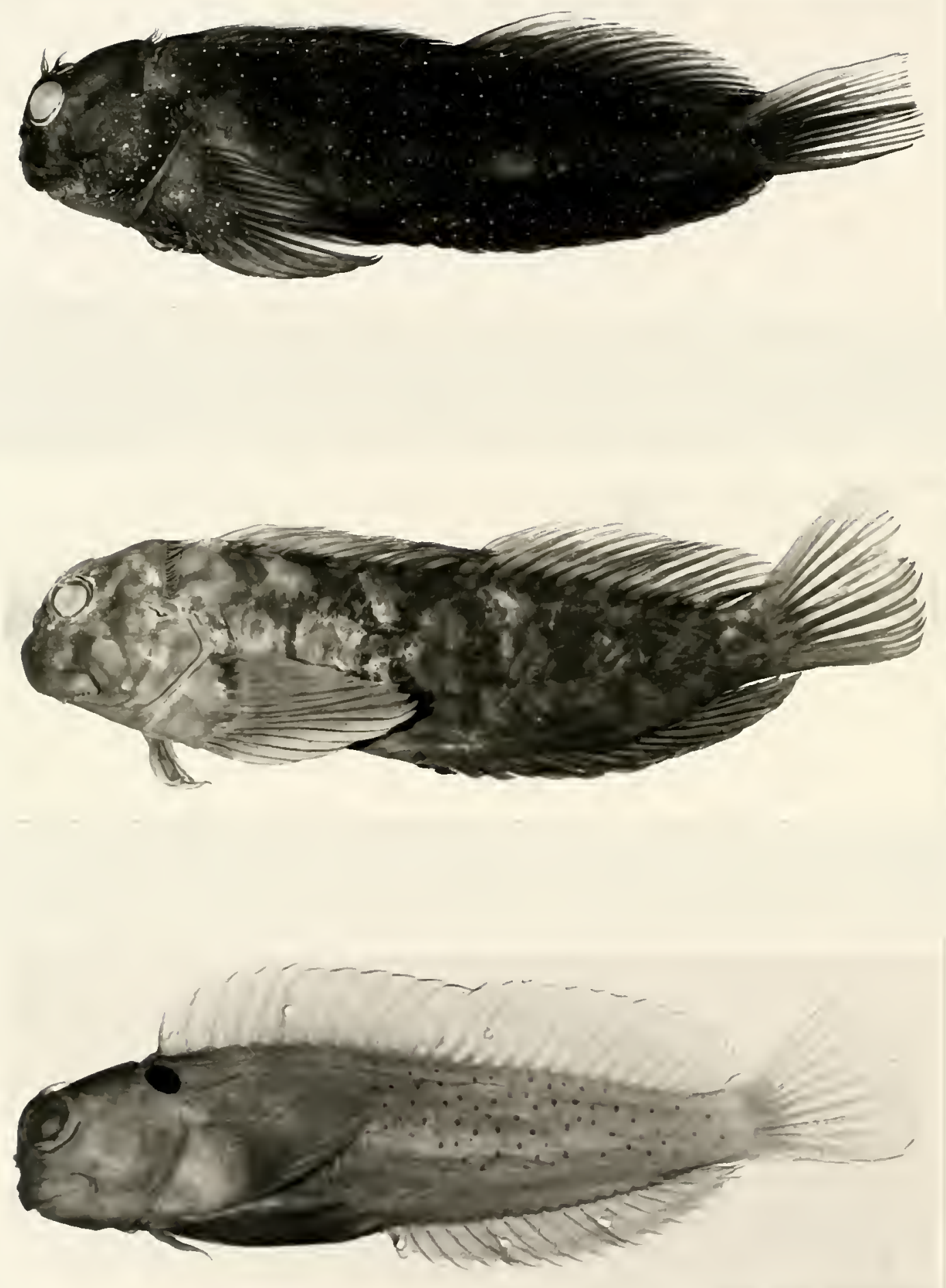
Figure 30. Male Cirripectes castaneus, Queensland, Australia (USNM 261272: $59 \mathrm{~mm}$ SL).

Figure 31. Reticulate color pattern of female Cirripectes castaneus, Queensland, Australia (USNM 261272: $60 \mathrm{~mm}$ SL).

Figure 32. Uniformly brown color pattern of female Cirripectes castaneus, Queensland, Australia (USNM 228189: $54 \mathrm{~mm}$ SL). 

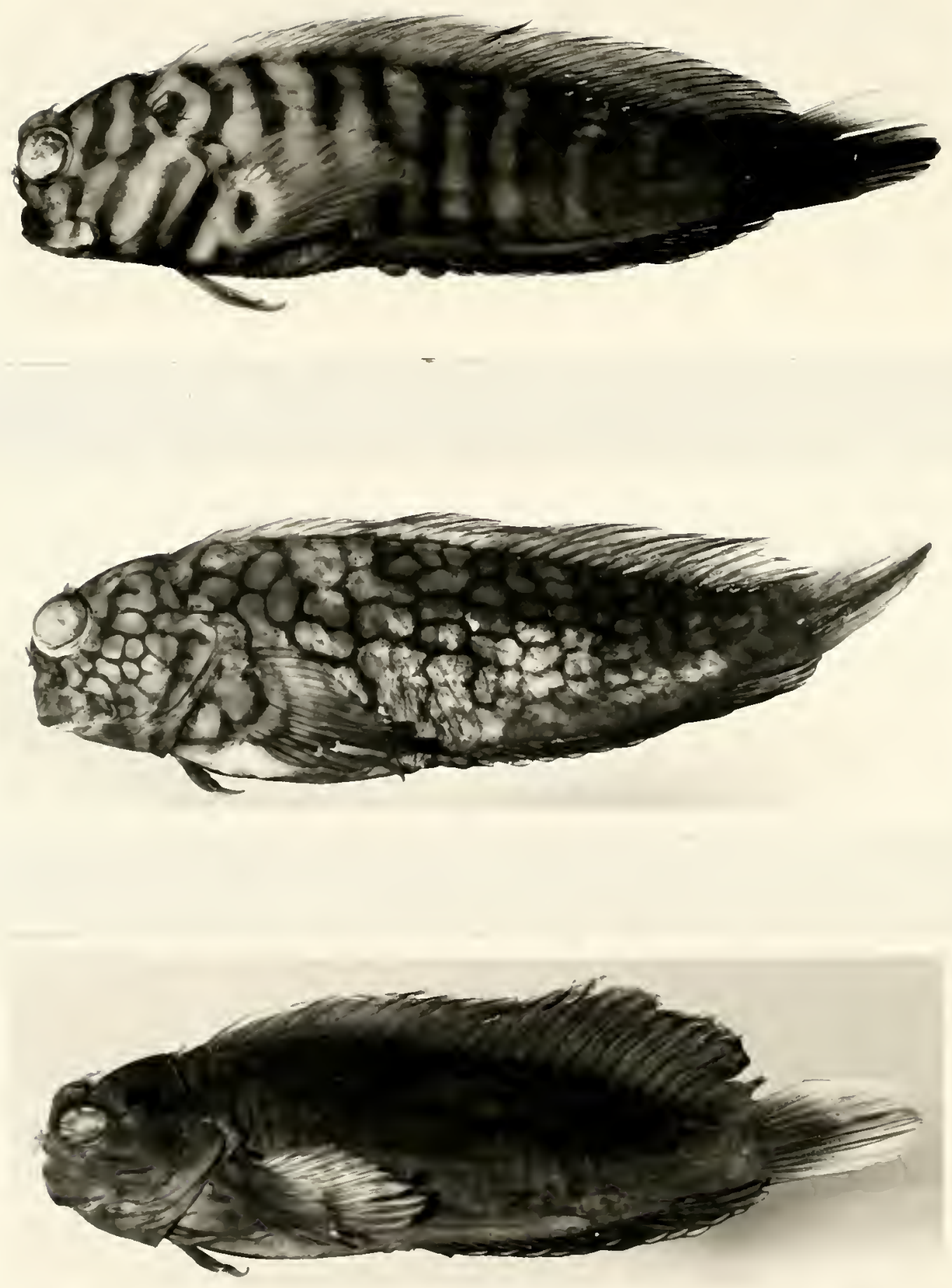
Figure 33. Male Cirripectes chelomatus, Queensland, Australia (USNM 258766: $83 \mathrm{~mm}$ SL).

Figure 34. Male Cirripectes filamentosus, Northern Territory, Australia (NTM uncat.: $41 \mathrm{~mm}$ SL). 

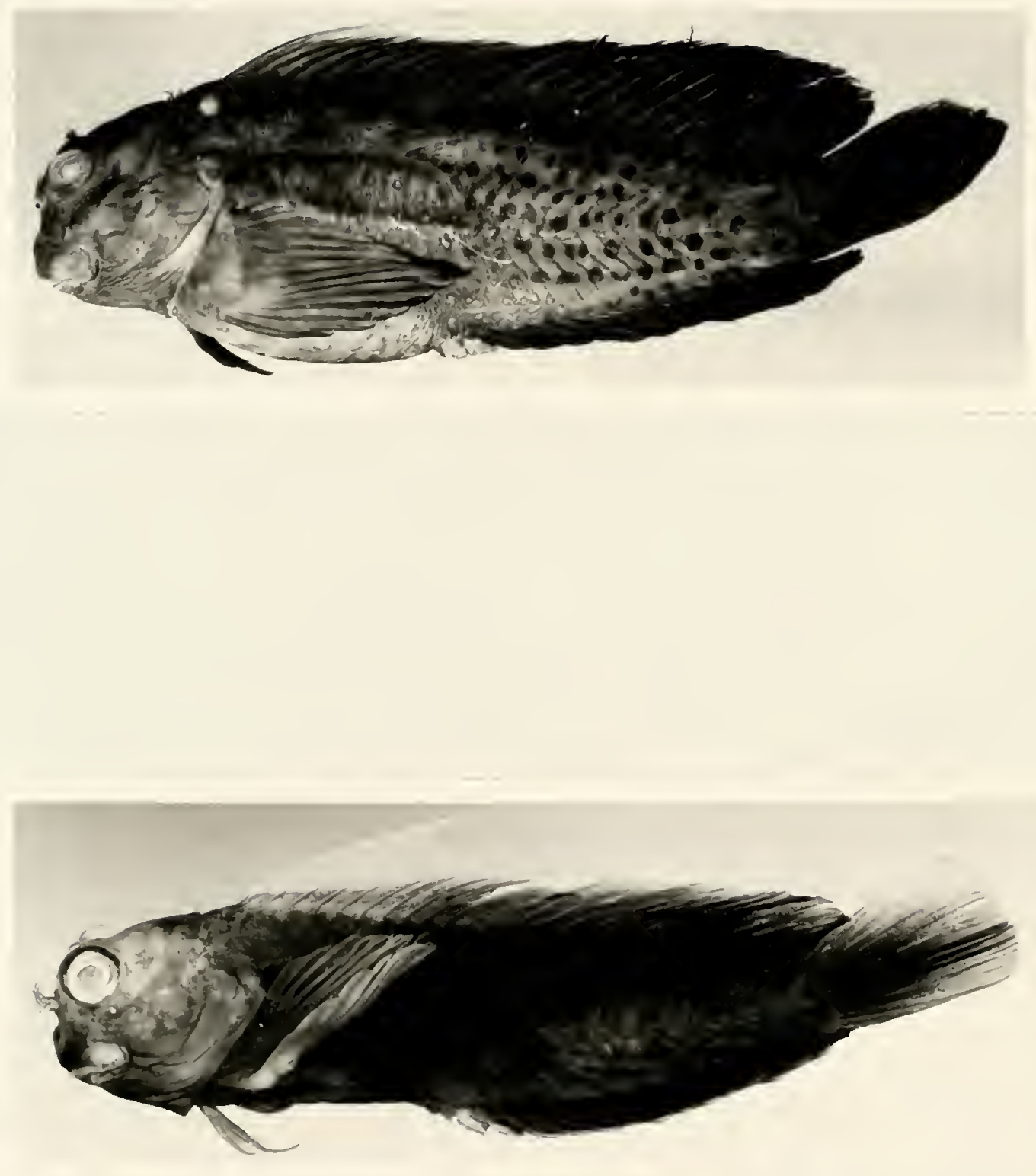
Figure 35. Young male Cirripectes fuscoguttatus, Caroline Is lands (CAS 48937: $39 \mathrm{~mm} \mathrm{SL}$ ).

Figure 36. Male Cirripectes fuscoguttatus, Marshall Islands (BPBM 8008: $93 \mathrm{~mm} \mathrm{SL).}$ 

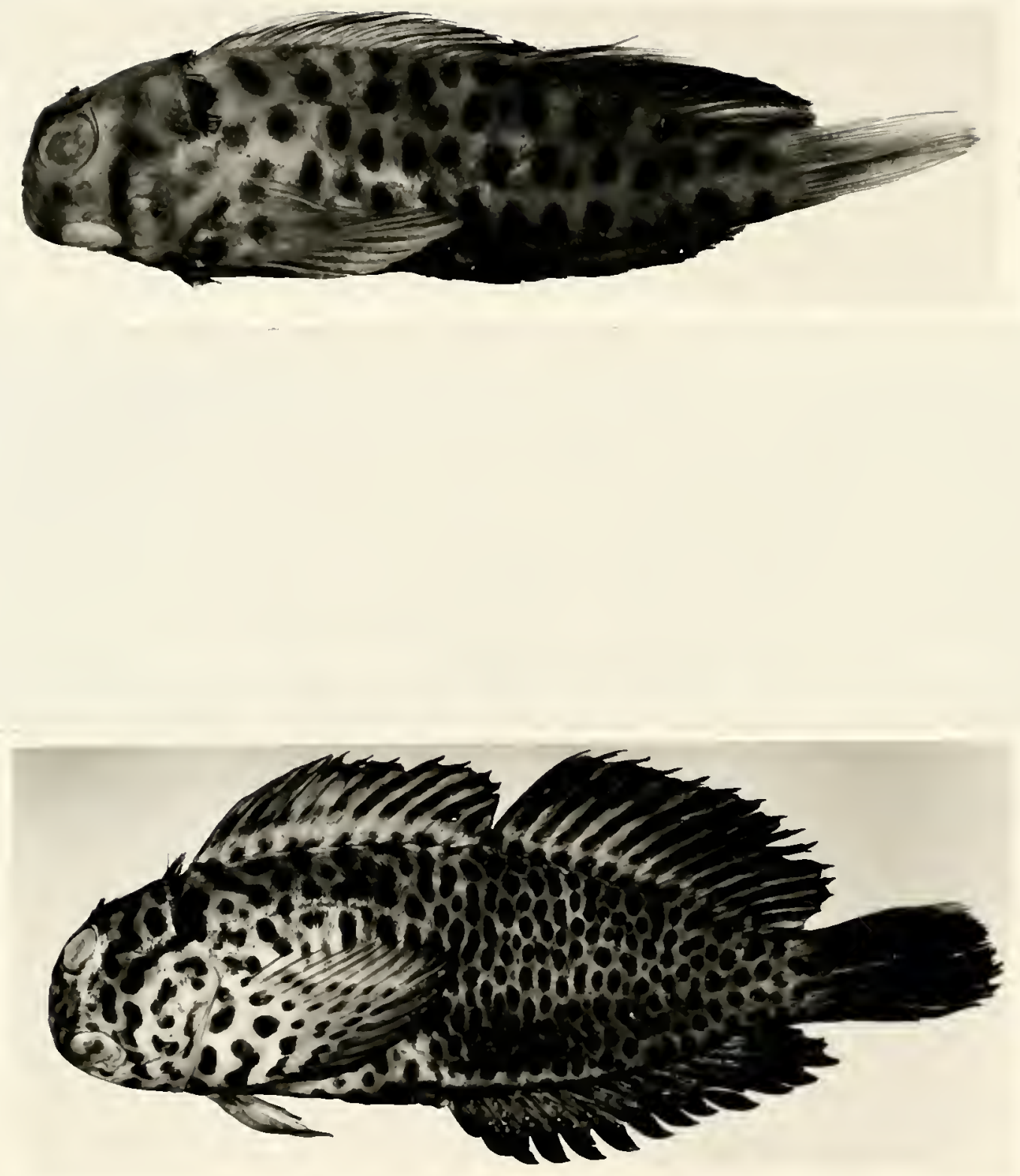
Figure 37. Male Cirripectes gilberti, holotype, Sumatra (USNM 274749: $82 \mathrm{~mm}$ SL).

Figure 38. Female Cirripectes gilberti, paratype, Chagos Archipelago (ROM 46597: $90 \mathrm{~mm}$ SL).

Figure 39. Young female Cirripectes gilberti, Cocos-Keeling Atoll (ANSP 134747: $38 \mathrm{~mm}$ SL). 

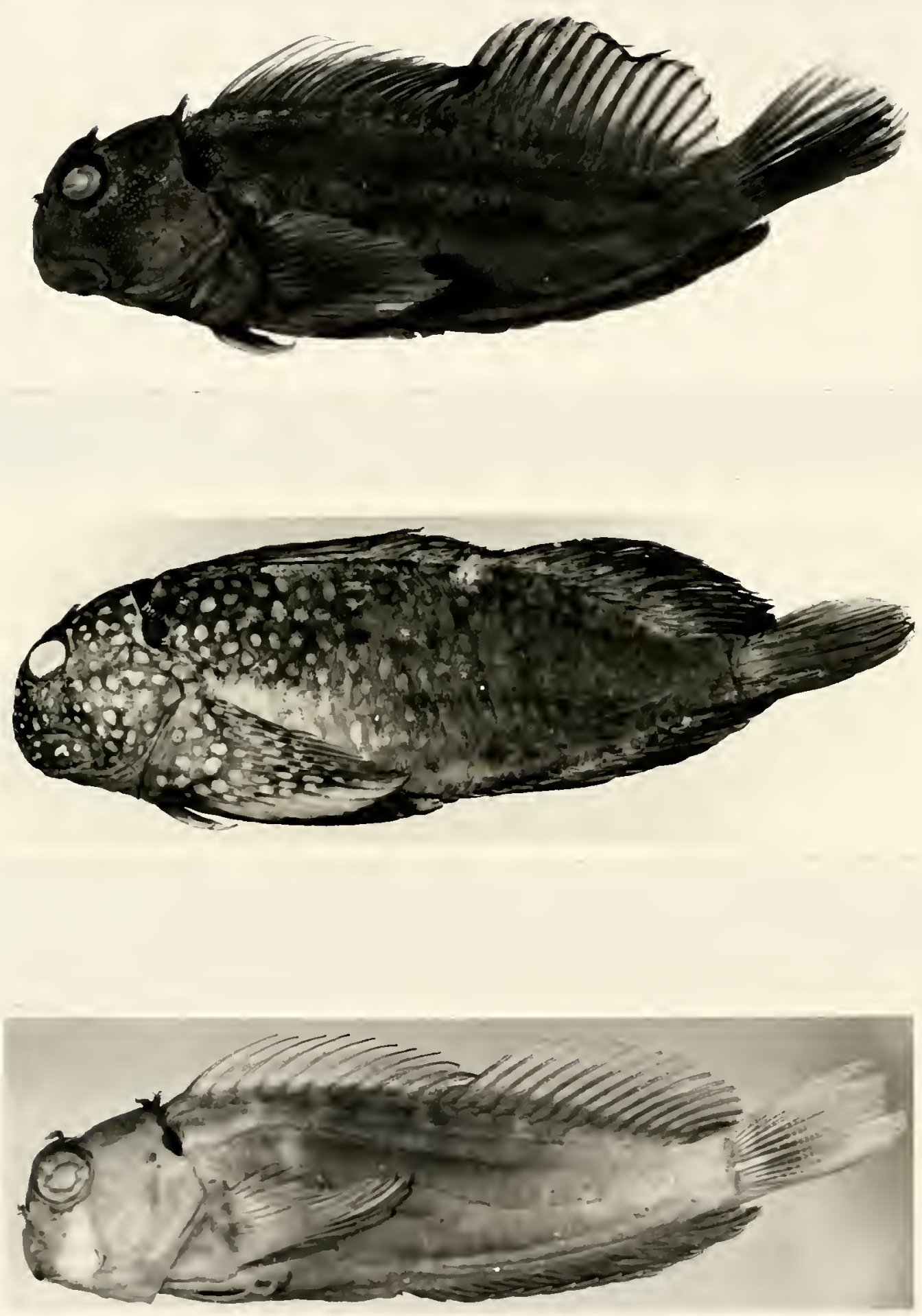
Figure 40. Female Cirripectes hutchinsi illustrating barred color pattern of speciemens from the northern end of the geographinc range; Northwest Cape, Western Australia (WAM P.25374-042: 69 min SL).

Figure 41. Female Cirripectes hutchinsi from the southern end of the range; Rottnest Island, Western Australia (AMS I.20243-004: $81 \mathrm{~mm}$ SL).

Figure 42. Male Cirripectes hutchinsi, holotype, Rottnest Island, Australia (WAM P. 25744-003: $90 \mathrm{~mm} \mathrm{SL}$ ). 
Figure 43. Male Cirripectes imitator, Ogasawara Islands (FAKU 48203: $67 \mathrm{~mm}$ SL).

Figure 44. Female Cirripectes imitator, Ogasawara Islands (FAKU 48187: $62 \mathrm{~mm}$ SL). 

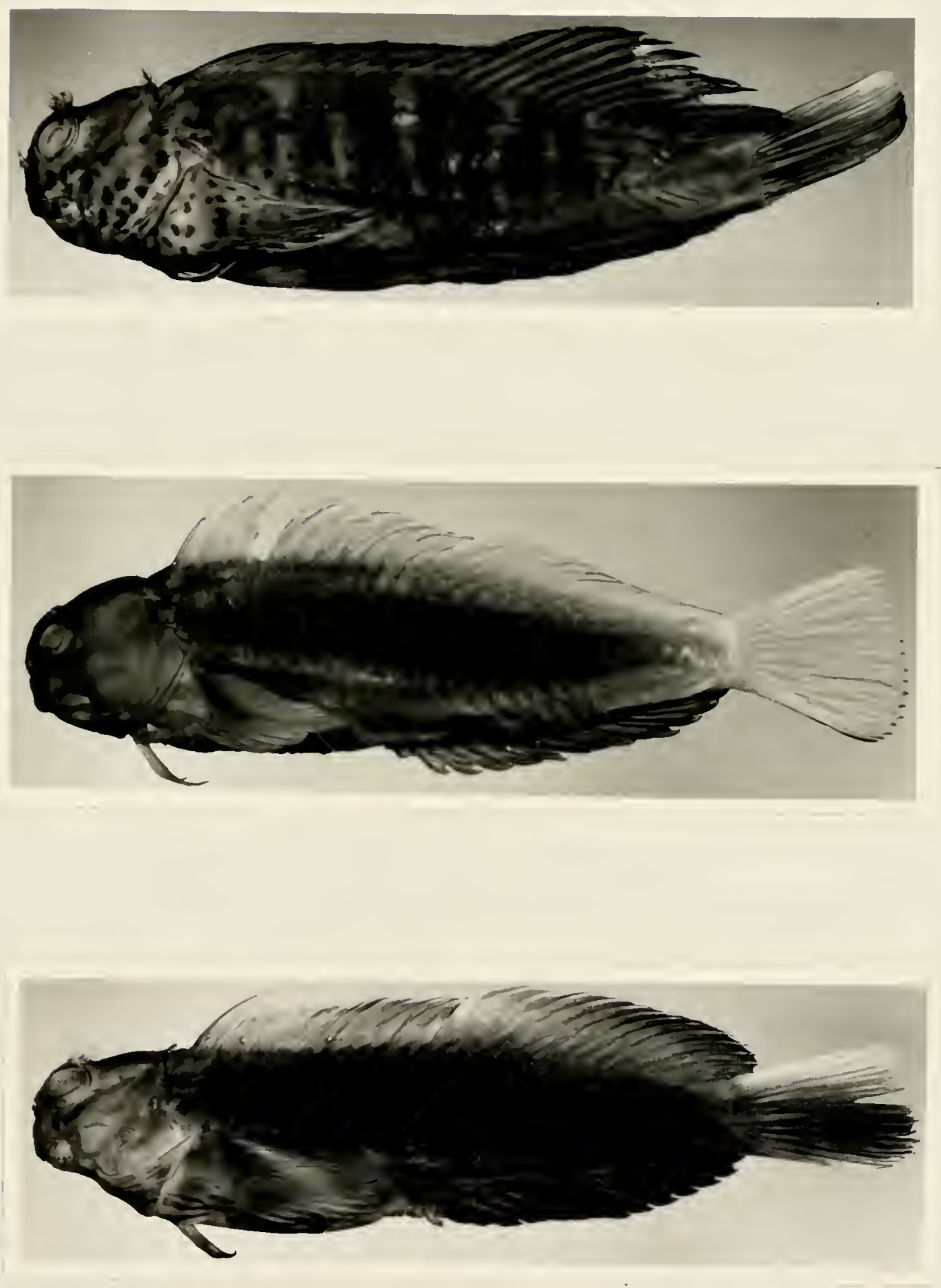

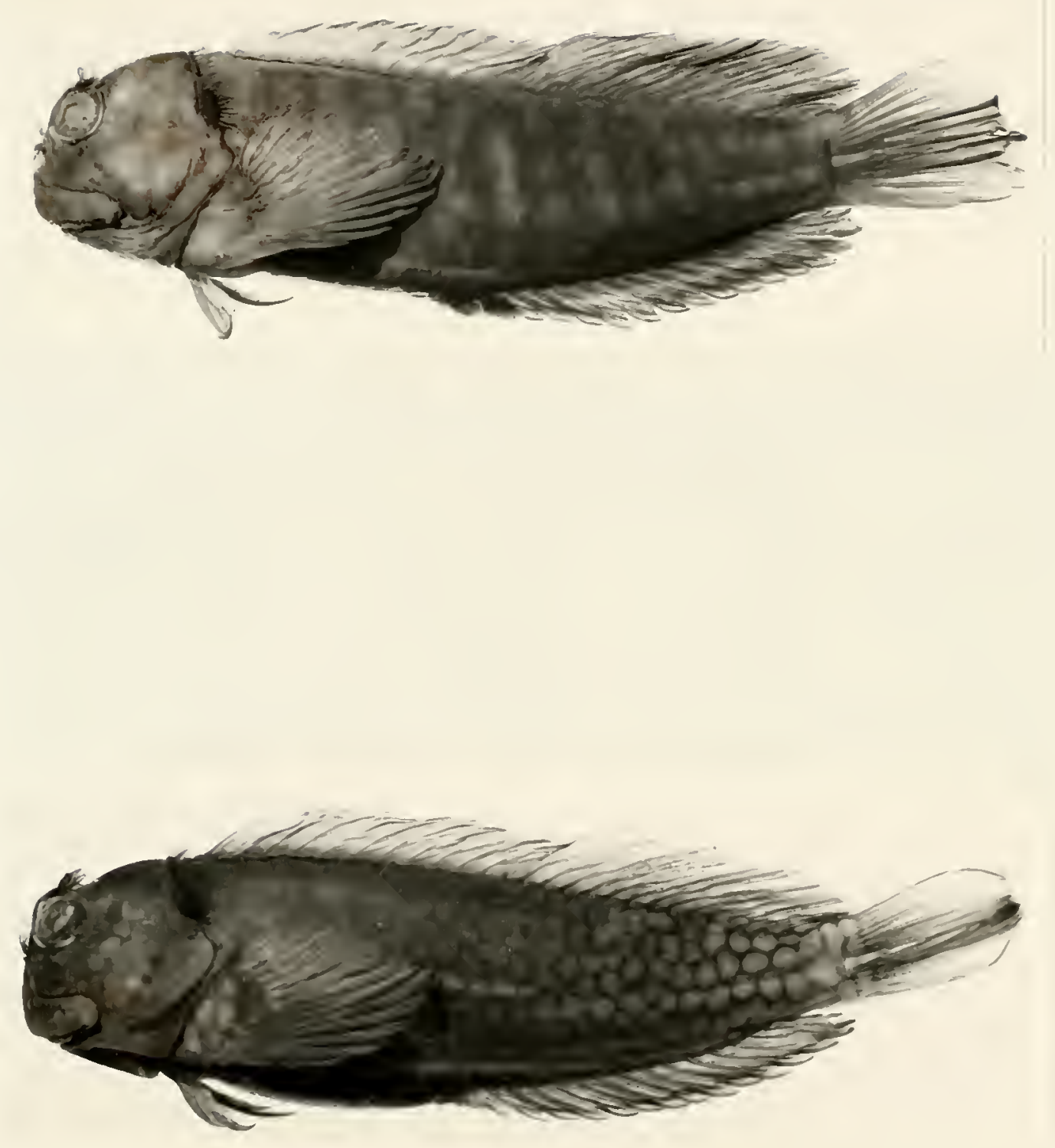
Figure 45. Male Cirripectes jenningsi, Line Islands (USNM 200615: $75 \mathrm{~mm}$ SL).

Figure 46. Female Cirripectes kuwamurai, holotype, Japan (FAKU 48479: $55 \mathrm{~mm} \mathrm{SL).}$

Figure 47. Male Cirripectes obscurus, Hawaiian Islands (BPBM uncat.: $106 \mathrm{~mm}$ SL). 

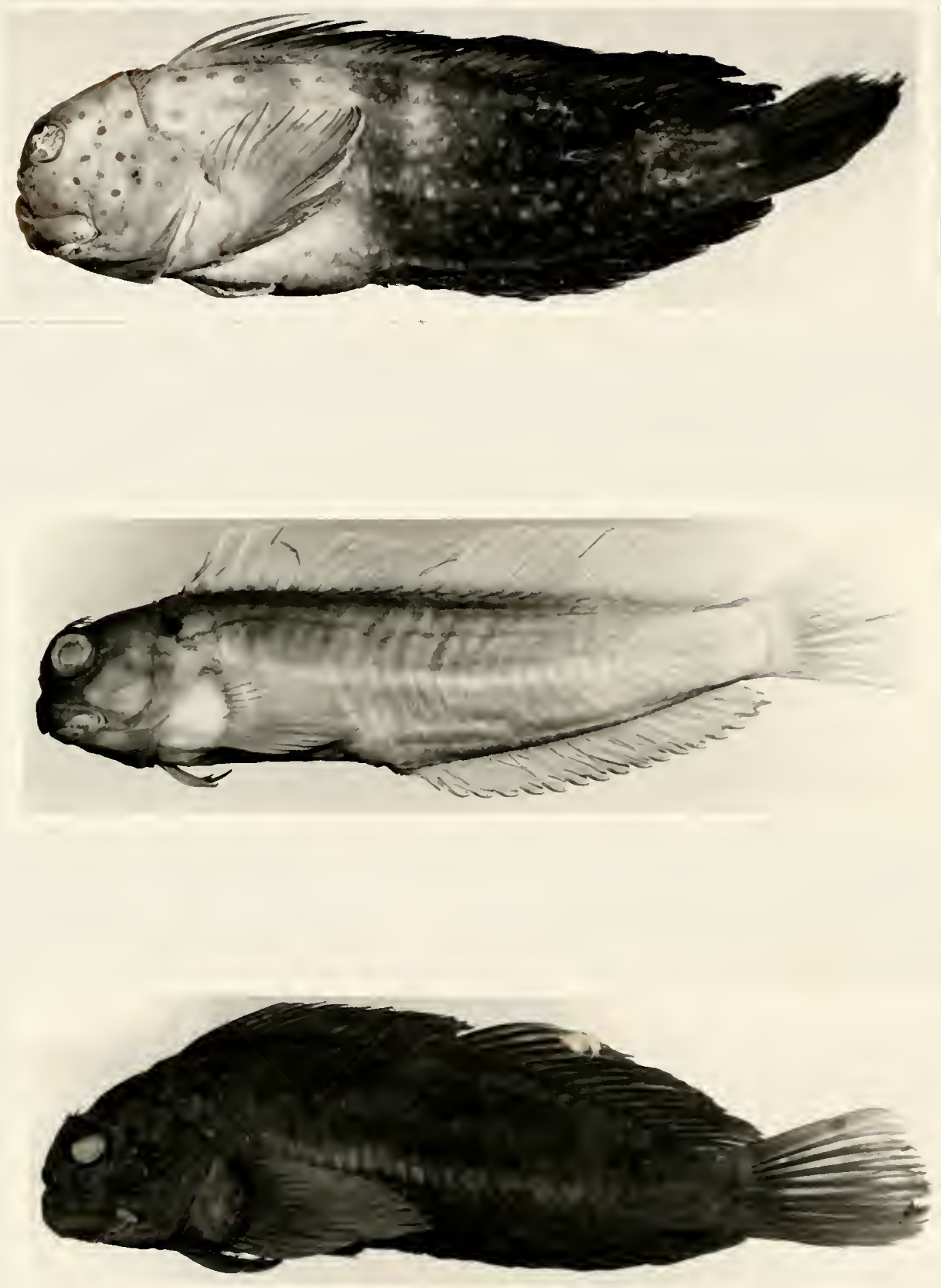
Figure 48. Female Cirripectes obscurus, Hawaiian Islands (BPBM uncat.: $109 \mathrm{~mm}$ SL).

Figure 49. Male Cirripectes perustus, Caroline Islands (CAS 38843: $44 \mathrm{~mm} \mathrm{SL).}$

Figure 50. Female Cirripectes perustus, Philippine Islands (USNM 228272: $62 \mathrm{~mm}$ SL). 

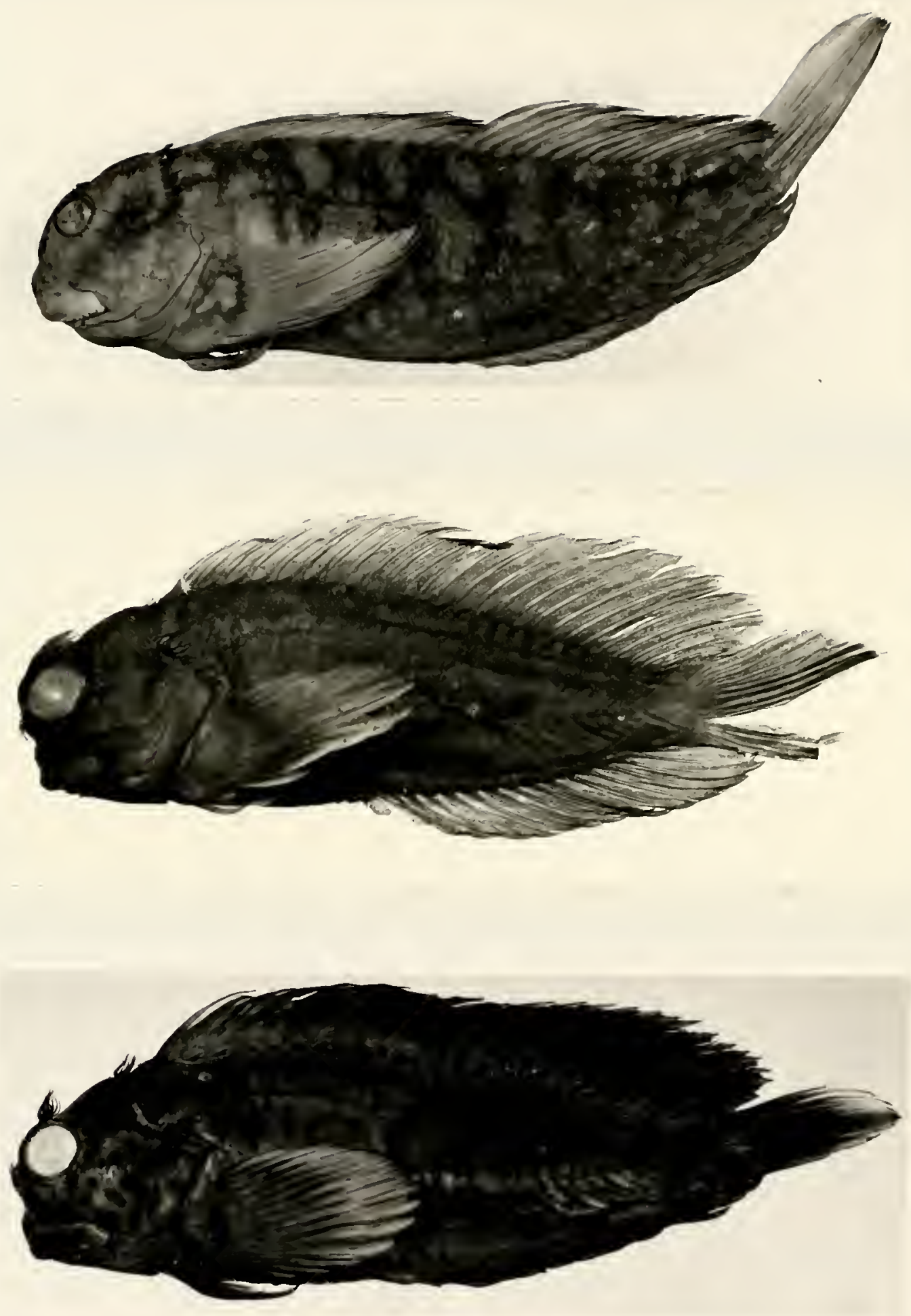
Figure 51. Young Cirripectes polyzona, Phoenix Islands (USNM 274295: 23 mm SL).

Figure 52. Female Cirripectes polyzona, Papua-New Guinea (USNM 274291: $45 \mathrm{~mm}$ SL). 

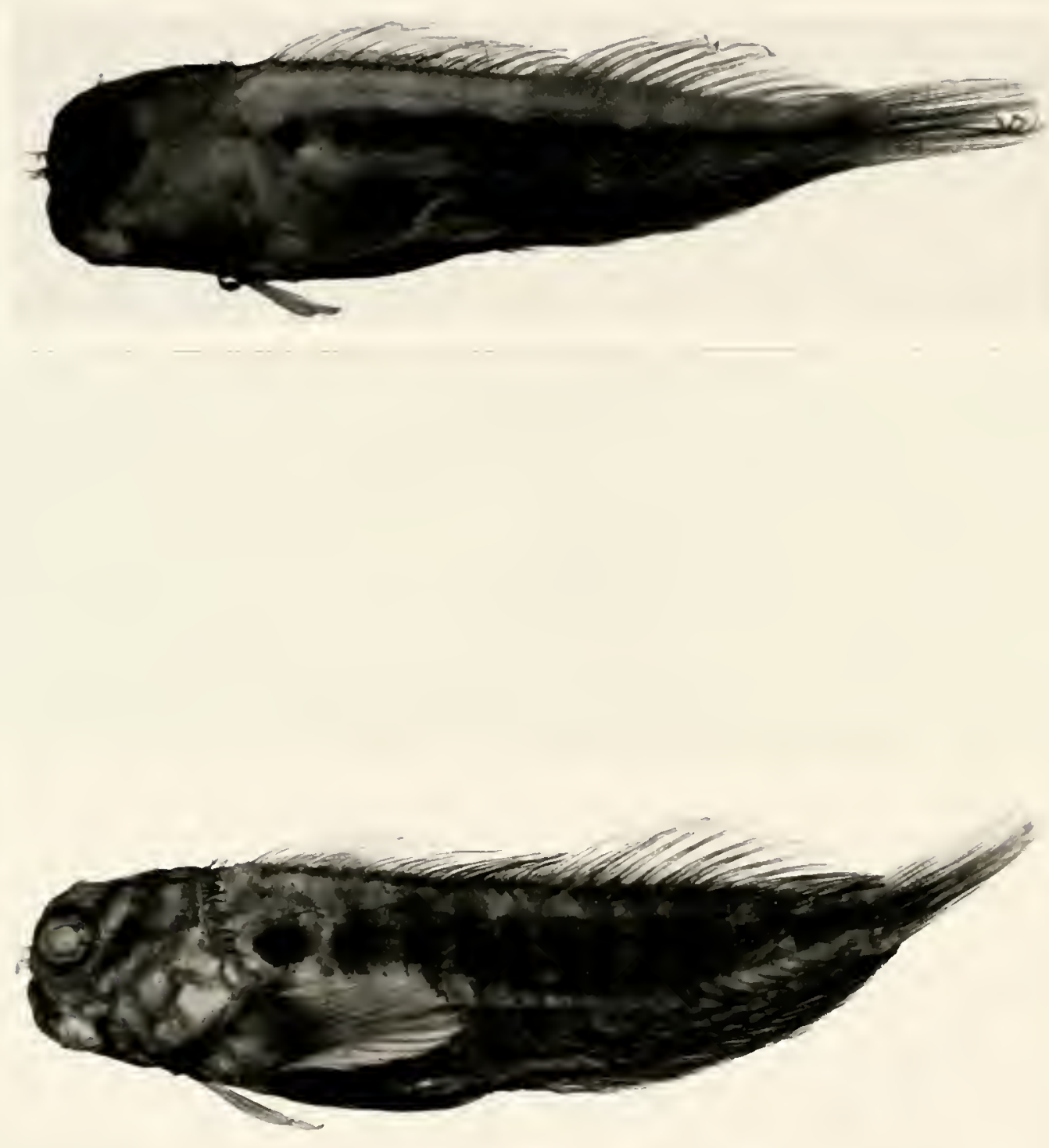
Figure 53. Male Cirripectes quagga exhibiting spotted color pattern, Line Is lands (USNM 199460: $67 \mathrm{~mm} \mathrm{SL).}$

Figure 54. Female Cirripectes quagga exhibiting slightly barred

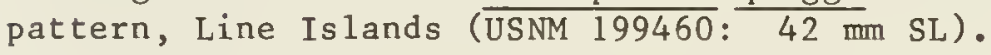

Figure 55. Male Cirripectes quagga exhibiting pale-peduncle color pattern, Samoa (UF uncut.: $41 \mathrm{~mm}$ SI). 

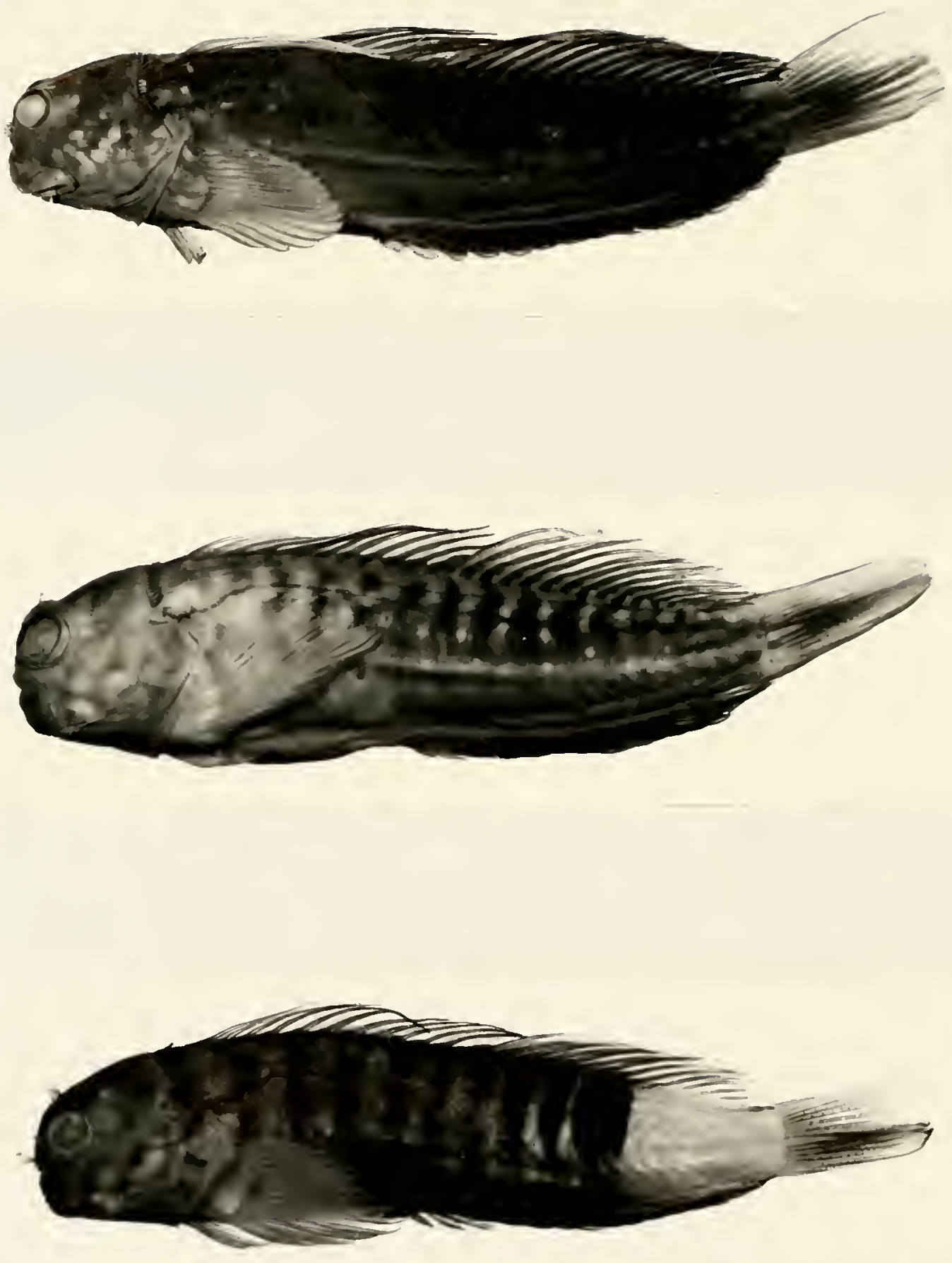


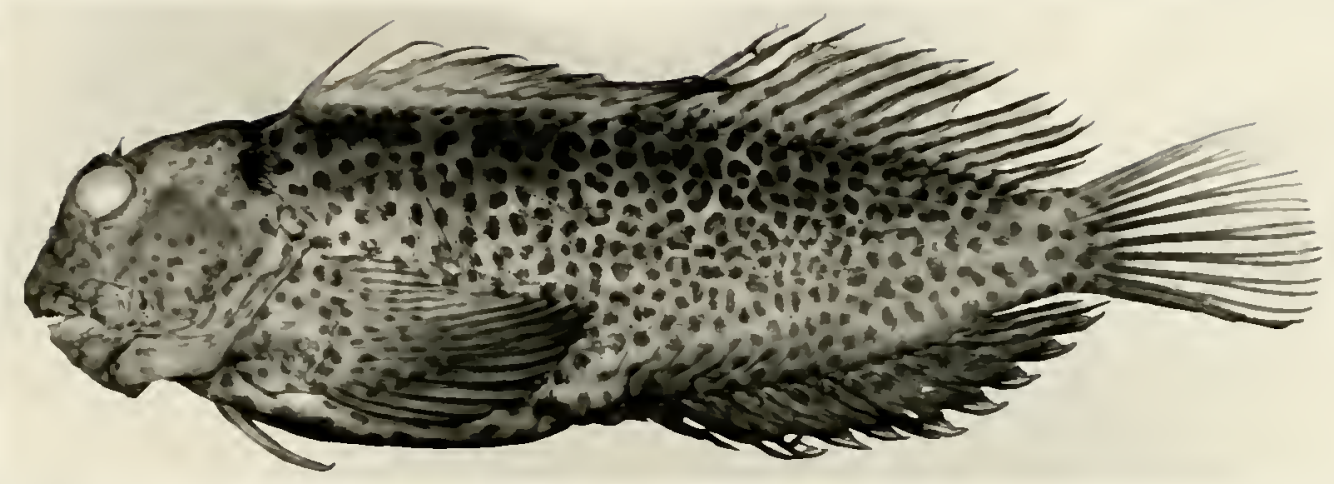

Figure 56. Female Cirripectes randalli, paratype, Mauritius (RUSI 22306: $79 \mathrm{~mm} \mathrm{SL).}$

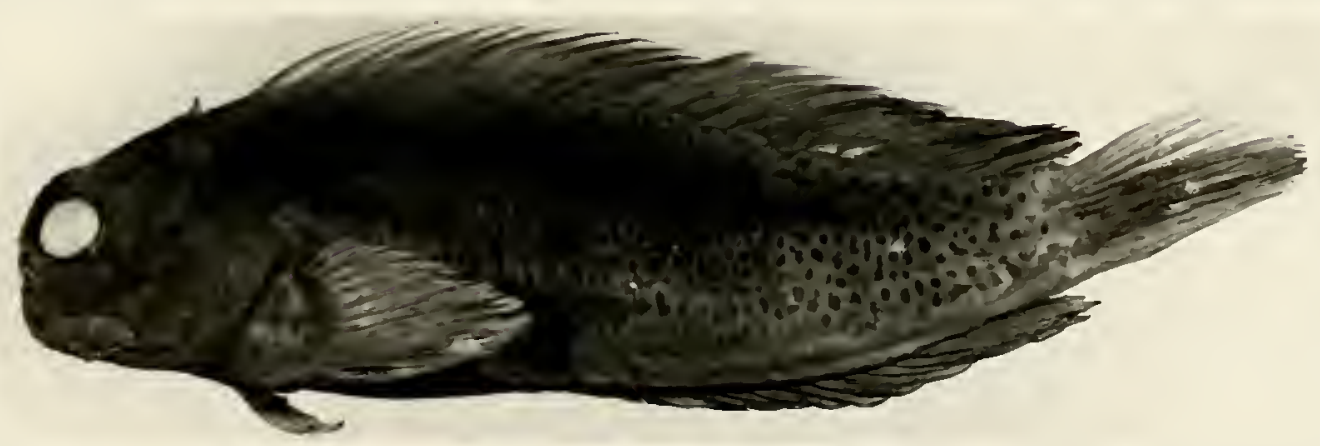

Figure 57. Male Cirripectes randalli, holotype, Cargados Carajos Shoals (USNM 227873: $92 \mathrm{~mm} \mathrm{SL}$ ). 
Figure 58. Male Cirripectes springeri, paratype, Papua-New Guinea (USNM 228292: $54 \mathrm{~mm}$ SL).

Figure 59. Female Cirripectes stigmaticus, Queensland, Australia (AMS I.19473-095: $83 \mathrm{~mm} \mathrm{SL).}$

Figure 60. Male Cirripectes stigmaticus, Caroline Islands (USNM 223505: $60 \mathrm{~mm} \mathrm{SL).}$ 

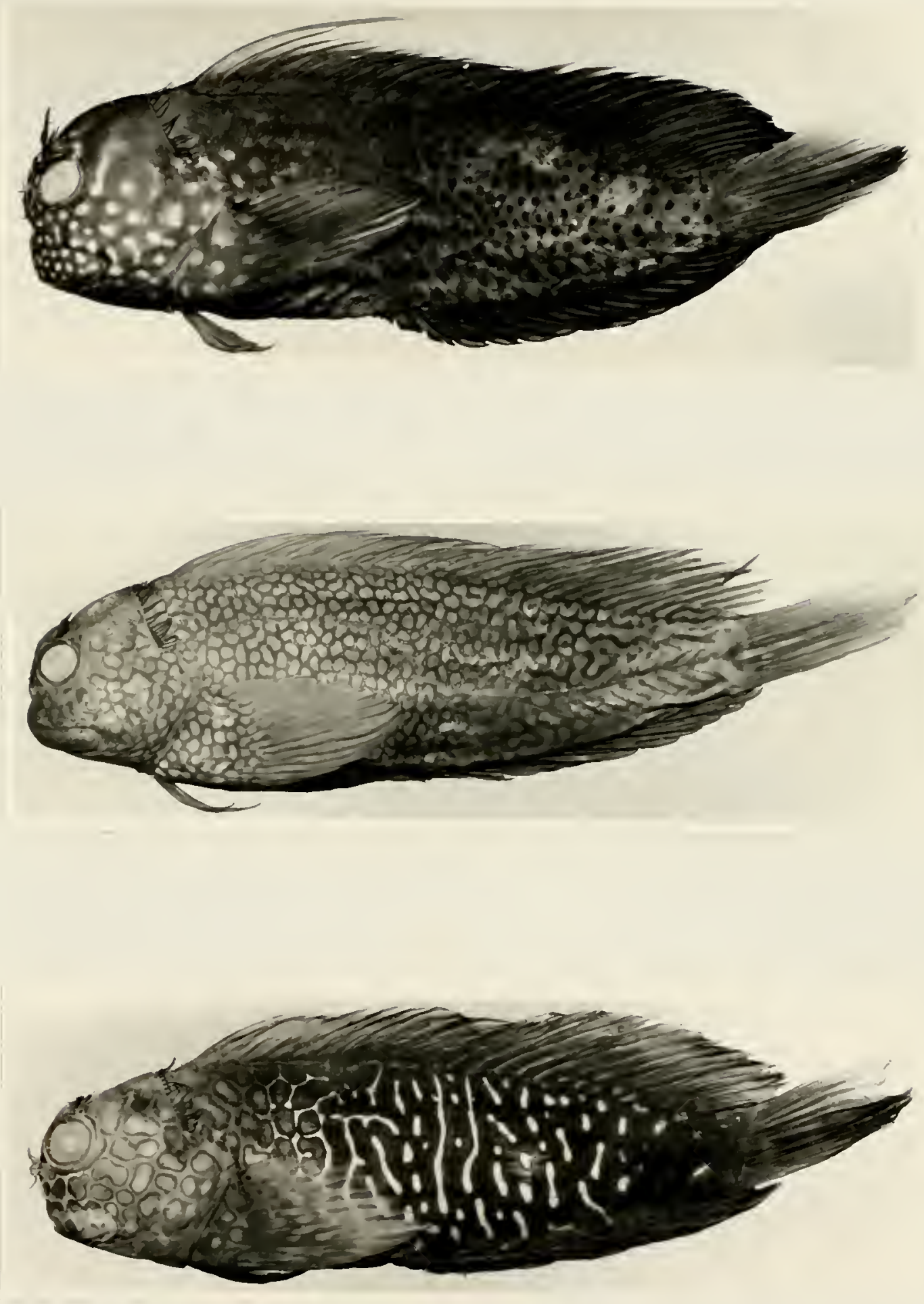
Figure 61. Male Cirripectes vanderbilti, Hawaiian Islands (USNM 205336: $53 \mathrm{~mm}$ SL).

Figure 62. Male Cirripectes variolosus, Pacific Ocean (USNM 198731: $61 \mathrm{mn}$ SL).

Figure 63. Female Cirripectes variolosus exhibiting the spotted

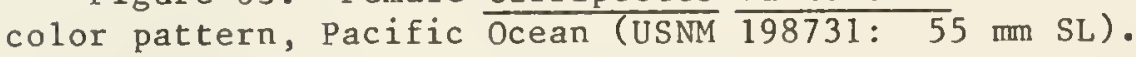



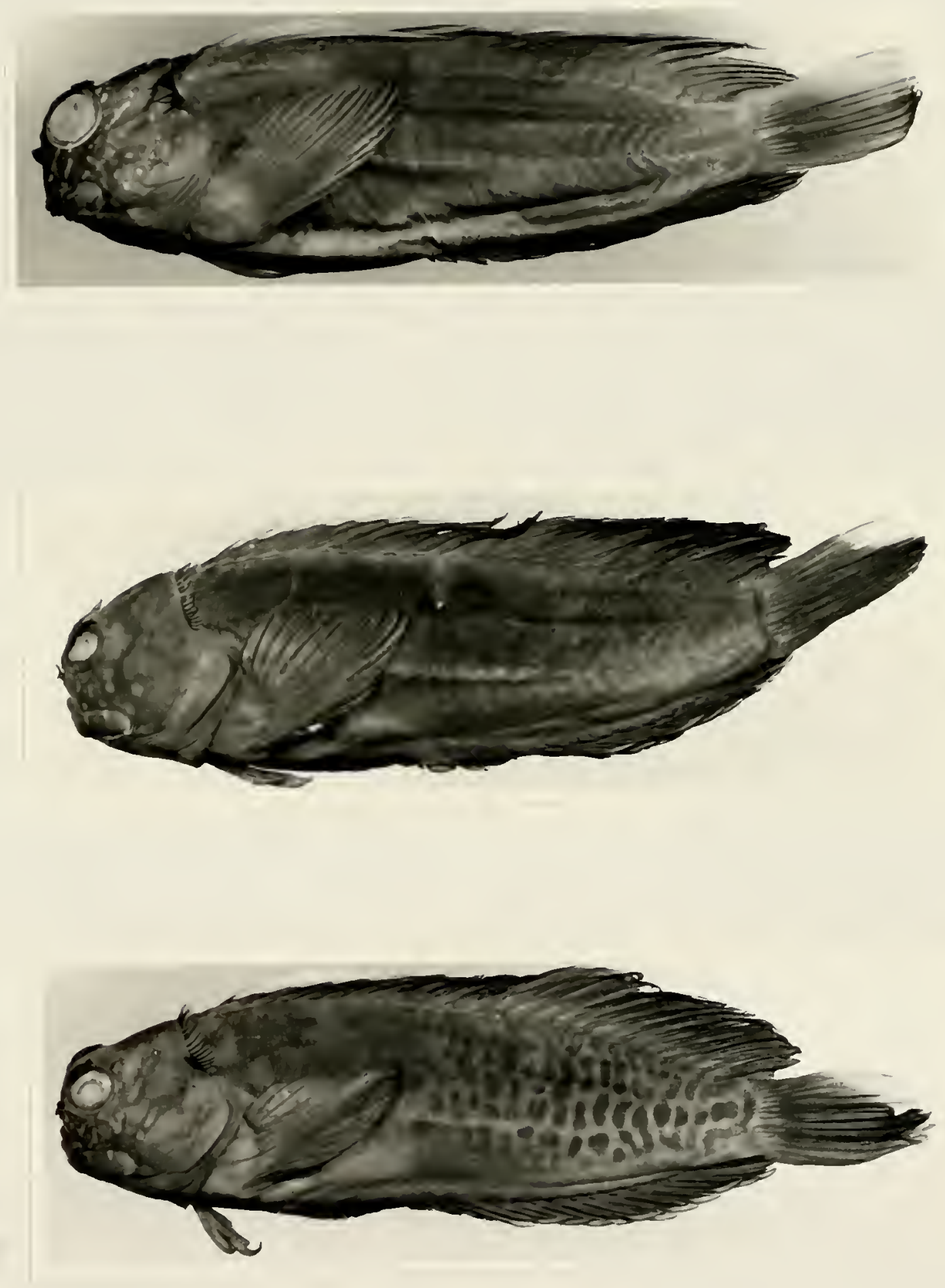
Cirripectes alboapicalis (Ogilby)

Salarias alboapicalis Ogilby, 1899:742 (Lord Howe Island). Cirripectus variolosus patuki De Buen, 1963:58 (Easter Island).

Diagnosis. A species of Cirripectes with the following characters: small scalelike flaps over anterior portion of lateral line, dark spot on head behind eye, 11 or more lateral-line tubes with last tube positioned before caudal-fin base, and tiny white or brown spots on body. It occurs south of $20^{\circ} \mathrm{S}$ latitude in the pacific Ocean.

Description. Dorsal fin XII, 15-17 ( 16 in $85.7 \%$ of specimens); anal fin II, $16-18$ ( 17 in $84.1 \%$ ); total procurrent caudal-fin rays 10-14; pelvic fin 1,4 ; vertebrae $10+21-23(22$ in $95.7 \%)=31-32$ (32 in 95.7\%); last pleural ribs on vertebral centrum 12; last epipleural rib on vertebral centrum 18-23 (19-22 in 96.5\%); anal pterygiophores $1-1-1$, $1-1-2$, or $\hat{1}-\hat{2}-1$ (1-1-2 in $86.2 \%)$; nuchal cirri $28-42$ (32-39 in $95.5 \%)$; supraorbital cirri 4-18 (5-12 in 96.4\%); nasal cirri 8-27 (8-17 in $91.7 \%)$; LL tubes $9-17$ ( $11-17$ in $95.9 \%)$; last LL tube positioned at point between verticals from dorsal-fin ray 12 and caudal-fin base ( 14 to caudal-fin base in $96.2 \%$ ); irregularly spaced scalelike flaps along anterior part of lateral line; lower lip crenulate mesially (plicate 
laterally); gill rakers 25-31 (based on 68 specimens); pseudobranchial filaments on one side $7-13$ (based on 79 specimens); premaxillary teeth 200-302 (based on 22 specimens; highest numbers in largest specimens); dentary teeth 96-146 (based on 22 specimens); upper 1 ip crenulae approximately 34-52; nuchal cirri in two groups that do not overlap at midpoint of nape, no nuchal flaps (type E; Figure 7); first dorsal-fin spine of adults equal to or slightly longer than second $(0-2.1 \mathrm{~mm}$ longer in males, 0-2.0 mm longer in females); dorsal-fin membrane deeply incised above last dorsal-fin spine; dorsal-fin membrane attached to caudal peduncle in advanue of base of caudal fin; cephalic pore system complex ( 4 or more pores at most positions; Figure 17; number of pores increases with increasing SL); mid-snout pores present (Figure 17); extra interorbital pore position present (Figure 17); two pore positions behind nuchal flap (Figure 17); male genital papilla with urogenital orifice located at distal tip or on posterior side of a single slender filament (less than 1.0 m long) on a fleshy swelling behind anus (types III or IV; Figure 13); testes elongate, length equals 2-3 times width; maximum size about $125 \mathrm{~mm}$ SL; ophioblennius larvae with 2-4 canines posteriorly on each dentary.

The smallest mature female (ova about $0.5 \mathrm{~mm}$ diameter) examined was about 50 man SL. Males mature by about $50 \mathrm{~mm}$ SL.

Color in alcohol. Ophioblennius stage larvae (based on two specimens, 33 and 35 m.m SL, from Rapa) with pale-brown head and body; dark spot present posteriorly on each side of lower lip and at base of symphyseal canines inside mouth; all fins pale; all cirri brown. 
A 34 SL specimen from Rapa retains the larval dentition, but has the dark spot behind the eye, pale tips of dorsal-fin spines, and tiny spots on body that are typical of adults.

Typical color pattern (Figure 27) of young and adults of both sexes consists of brown background color with tiny white or dark-brown spots over head and some or all of body; dark-brown pupil-sized spot on each side of head behind orbit; spinous-dorsal fin with tips of anterior spines pale, remainder of fin brown; caudal fin dusky; anal fin frequently with pale distal margin, remainder of fin dark brown, usually with tiny pale spots; pectoral fin dusky with dark area at bases of dorsal rays. Some males and females have a mottled pale-and-brown color pattern overlaid with tiny pale or dark brown spots.

Color in life. Similar to color in alcohol except as follows (based on a color slide of a specimen from Lord Howe Island, and an Easter Island specimen figured by Springer [1970]): tips of dorsal-fin spines and upper caudal-fin rays reddish orange; anal fin bluish black with tips of rays bluish white; iris with narrow inner yellow ring, remainder black.

Comparisons. Cirripectes alboapicalis and $\underline{C}$. obscurus differ from their congeners in having small scalelike flaps over anterior portion of lateral line and a dark spot on head behind eye. These two species are allopatric and have an antitropical distribution pattern, with $\underline{\mathrm{C}}$.

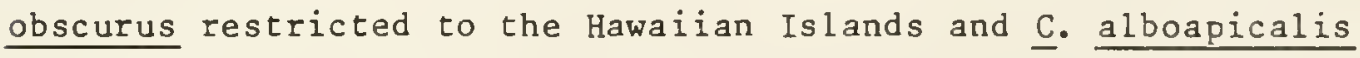
occurring south of $20^{\circ} \mathrm{S}$ latitude in the Pacific Ocean. Adult $\underline{C}$.

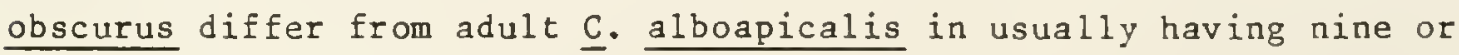




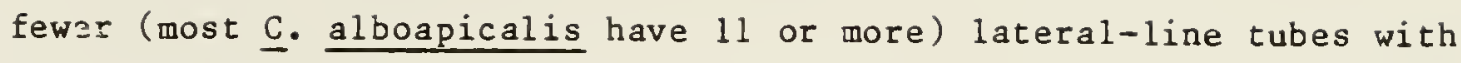
the last tube (or the end of the continuous canal if there are no tubes) on the caudal-fin base (versus usually before the caudal--fin base). This combination of few or no lateral-line tubes with a posterior termination point differentiates all but one of the available specimens of $\underline{C}$. obscurus and $\underline{C}$. alboapicalis. Although this specimen, a male, is the only specimen from the Cook Islands, it clusters with $\underline{C}$. obscurus in having nine lateral-line tubes with the last located on the caudal-fin base and a high number of supraorbital cirri, it associates with C. alboapicalis geographically and in having fewer (14) nasal

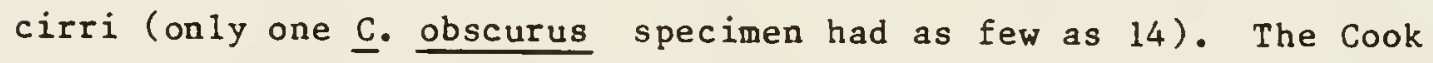
Islands male differs from males of both species in having a mottled color pattern (Figure 28), typical of females of the two species, but lacks the small pale spots seen on females. The mottled pattern has not been observed on any other adult males but may be related to size, as this is the largest specimen (about $125 \mathrm{~mm}$ SL) of Cirripectes alboapicalis examined. I tentatively identify the Cook Islands maie as C. alboapicalis, primarily because its collection locality is in the middle of the geographic range of this species. Additional specimens are needed to determine the correct placement of the Cook Islands male. Distribution. Cirripectes alboapicalis occurs in the South Pacific Ocean below $20^{\circ}$ south latitude. It is known to occur at Easter Island, Pitcairn Group, Tubuai Islands, Cook Islands, Kermadec Island, Norfolk Isiand, Lord Howe Island, and Capricorn Group of the Great Barrier Reef (Figure 19). This distribution pattern comprises, and extends to eastern Australia, Rehder's (1981) Pitcairn-Kermadec "Province.:: 
Etymology. The specific epithet is a combination of the Latin albus, meaning white, and apicalis, meaning at the apex, and refers to the pale area at the tips of the dorsal-fin spines.

Comments. Counts and measurements do not vary geographically. The degree of spotting on the body is variable within collections from a single locality, ranging from a dense covering of tiny white spots to no apparent spots (lack of spots seen only in preserved specimens).

Allen et al. (1976) state that Cirripectes alboapicalis, in addition to other characters, is distinct "in lacking a median nuchal pore." The specimens I examined have at least one pore (usually three or more) at that position.

Nomenclatural discussion. Ogilby (1899) based his description of Salarias alboapicalis on a $53 \mathrm{~mm}$ specimen from Lord Howe Is 1 and and mentioned other specimens, initially identified (Ogilby, 1889) as Salarias variolosus, measuring up to $125 \mathrm{~mm}$. Ogilby (1899) also stated that the specimen figured in Gunther (1877) as $\underline{S}$. variolosus is the same as his new species. The specimen Gunther figured is actually Cirripectes quagga. McCulloch and McNeill (1918) stated that the dorsal- and anal-fin ray counts made by Ogilby (1899) were in error. My findings support their finding. Strasburg (1956) stated that the holotype of $\underline{C}$. alboapicalis was lost. I examined 12 specimens in four lots from the Australian Museum that are designated as syntypes. As Ogilby (1899) did not specifically designate a holoiype (although he reiers to a $53 \mathrm{~mm}$ specimen), I concur with the Australian Museum's designation of these specimens as syntypes. One of these specimens (AMS I. 1863) is about $53 \mathrm{~mm}$ SL, and I designate this specimen as leciotype of Salarias alboapicalis. 
De Buen (1963) based his description of Cirripectus variolosus patuki on two specimens from Easter Island. I have not examined these specimens, but the description is sufficient to associate this subspecies with $\underline{\text { C. }}$ alboapicalis. Springer (1970) figured an Easter Island specimen and identified it as $\underline{\mathrm{C}}$. patuki, thereby recognizing this form at the species level. I have examined specimens collected at island groups between Lord Howe and Easter Is 1 and and have found no differences that warrant recognition of two species. Therefore, I place $\underline{C}$. patuki in the synonymy of $\underline{C}$. alboapicalis.

Material examined. Types: AMS I.1863 ( 1 specimen: 54 mm SL), lectotype of Salarias alboapicalis, Lord Howe Island, $31^{\circ} 33^{\prime} \mathrm{S}$, $159^{\circ} 05^{\prime}$ E, 12 July 1888; AMS I. 1861 (9: 26-33 m sL), paralectotypes of Salarias alboapicalis, same data as holotype. AMS I.1860 and I.1862 ( 98 and 71 , respectively), paralectotypes of Salarias alboapicalis, same data as lectotype.

Other material examined. Australia, Queensland: AMS I.17445-040 (1:73). Lord Howe Is 1 and: AMS I.4635 (1), AMS I.4É36 (1), AMS IB.5490 (1), AMS IB.5502 (1), AMS I.5900 (1), AMS I.5902 (1), AMS I.17373-006 (1), AMS I.17379-003 (1), AMS I.17388-003 (1), AMS I.17414-001 (1), AMS I. 17424-008 (26: 27-71), BPBM 14853 (7), CAS-SU 9206 (2). Norfolk Island: AMS I. 18497-008 (6), AMS I.20268-008 (9), AMS I.20271-005 (1), ANSP 75307 (5: 59-98). Kermadec Islands: AMS I.10452-3 (2: 36, 36). Cook Islands: CAS UNCAT. (1: 123). Iuouai Islands: AMNH UNCAT. (2: 36-58), AMNH UNCAT. (1: 33), AMNH UNCAT. (9:32-35), BPBM 12916 (2: $39,59)$, ВРВМ $13308(2: 41,46)$, ВРBM $13413(5: 33-48)$, ВРBM 17222 (8: 32-56), BPBM 17302 (4: 34-38). Oeno Ato11: BPBM 16482 (2: 38, 42$)$. 
Henderson Island: BPBM UNCAT. (1: 30), BPBM 17096 (15). Ducie Atoll: BPBM 12267 (2). Pitcairn Island: BPBM 16730(2:70, 83), BPBM 16974 (14: 35-58), BPBM 16996 (1). Easter Island: BPBM 6755 (3: 35-45), BPBM 6756 (21: 33-82), BPBM 6757 (26:34-92), LACM 6560-33 (3: 38-48), USNM $65412(2: 42-82)$, USNM 65413(1: 38$)$.

\section{Cirripectes auritus Carlson}

Cirripectes auritus Carlson, 1981:408 (Fanning Island, Line Islands).

Diagnosis. A species of Cirripectes with the following combination of characters: greatly expanded black nuchal flap on either side of nape, dorsal fin entire, supraorbital cirri 2-7, nasal cirri 4-6, and small dark spots posteriorly on body.

Description. Dorsal ín XII-XIII (XII in $92.3 \%$ of specimens),15-17 (16 in $61.5 \%$ ); anal fin II, 16-18 (17 in 69.2\%); total procurrent caudal-fin rays 10-13; pelvic fin $I, 4$; vertabrae 10 (11 in a specimen with an aberrant anterior centrum) $+21-23(22$ in $84.6 \%)=31-33$ (32 in $84.6 \%)$; last pleural ribs on vertebral centrum $11-12$ ( 11 in $84.6 \%$; last epipleural rib on vertebral centrum 17-19; anal pterygiophores 1-1-2 or 1-2-1; nuchal cirri 22-34; supraorbital cirri 2-7; nasal cirri 4-6; LL tubes 7-12; last LI tube positioned at point between verticals from dorsal-fin ray 5-13 ( $5-9$ in $83.3 \%$ ); no scalelike flaps along LL; lower lip smooth mesially (plicate laterally); gill rakers 21-25 (based on 4 specimens); pseudobranchial filaments on one side 8-10 (based on 4 specimens); premaxillary teeth 143-161 (based on Carlson, 1981); 
dentary teeth 64-80 (based on Car1son, 1981); upper 1ip crenulae approximately 34-39; nuchal cirri arrangement consisting of 5-8 independent cirri distributed between two greatly expanded nuchal flaps bearing minute cirri (type A; Figure 7); first dorsal-fin spine of adults approximately equal to second in males and females; dorsal-fin membrane very slightly or not incised above last dorsal-fin spine; dorsal-fin membrane attached to caudal peduncle in advance of base of caudal fin; cephalic pore system simple (1-2 pores at most positions; Figure 17); mid-snout pores present (Figure 17); extra interorbital pore position absent (Figure 17); pore positions behind nuchal flap $1-2$ ( 2 in $87.5 \%$; Figure 17); male genital papilla with urogenital orifice located basally between two widely separated slender filaments (less than $1.0 \mathrm{~mm}$ long) on a fleshy swelling behind anus (type I, Figure 13); testes bulbous, length equals width; maximum SL about 70 m;

ophioblennius larvae with 2 canines posteriorly on each dentary.

The smallest mature female (ova about 0.5 m diameter) examined was about $50 \mathrm{~mm}$ SL. Males mature by abuut $45 \mathrm{~m}$ SL.

Color in alcohol. Ophioblennius stage larva (based on a 28 an SL specimen from Christmas Island, Line Islands) with cream colored head and body; no dark spots around mouth; all fins transluscent; greatiy expanded nuchal flap and all cirri pale.

Adults of both sexes have body coloration (Figure 29) that can consist of pale, brown, or mottled pale-and-brown background, but always with tiny dark-brown spots on posterior half to three-fourths of body. Head with two pale bars extending from orbit onto upper 1 ip on each side, remainder of head brown with irregular pale bars and spots; 
underside of head with broad brown band across throat. Dorsal fin dusky with pale spots on spines and pale streak extending dorsoposteriorly from eaci ray base to midheight of interradial membrane. Caudal fin dusky with tips of dorsalmost rays pale. Anal fin brown. Pectoral and pelvic fins pale. Nuchal flap black with pale cirri, cirri dorsal to nuchal flap brown to black; supraorbital and nasal cirri brown. Males with pale rugosities on anal-fin spines. Color in life. Adults (based on color slides of specimens from Kenya, Thailand, and Fanning Island) with body coloration variable. Large Kenyan male (about 80 m SL; specimen not collected) rith head and anterior half of body with peach background color; head with greenish-white spots on snout, around eye, and on nape; abdominal area yellowish-orange; posterior half of body pink with tiny reddish-brown spots; dorsal fin dusky with pink spots on spinous portion, pink streaks on basal half of rayed portion, and narrow bluish-white margin; caudal fin pinkish; anal fin bluish grey with tips of rays blue; anal spine rugosities yellowish; nectoral fin pale yellow; pelvic fin white; nuchal flap purplish black, bearing tiny yellow cirri, cirri dorsad of flap brown; supraorbital and nasal cirri peach with pale-greenish tips; iris with yellow inner ring and irregular reddish-brown outer ring.

Females at Similan Island, Thailand have a strongly mottled pattern consisting of irregular reddish-brown and pinkish-white spots and streaks on head, and irieguiar broad brown bars with pinkish-white interspaces on body. Mid-body with small brownish-black spots ringed with reddish-brown, spots becoming black posteriorly on body. Dorsal 
fin with reddish-orange spots and streaks. Abdominal area white. Color of other structures as described for Kenyan male.

Fanning island females resemble Similan Island females except the pale areas on head are all faintly pinkish, and those on body are light brown or cream colored; spots and streaks in dorsal fin are pink.

Comparisons. Cirripectes auritus and $\underline{\text { C. kuwamurai differ from }}$ their congeners in having a greatly expanded black nuchal flap on either side of the nape, with independently based cirri in a row across the nape between the flaps (type A; Figure 7). Although large nuchal flaps are present in members of the $\underline{C}$. fuscoguttatus group, species in the $\underline{\text { C. }}$ fuscoguttatus group have well-developed cirri on the nuchal flaps and have the cirri between the nuchal flaps connected at their bases by a low basal membrane (type C; Figure 7). Cirripectes auritus

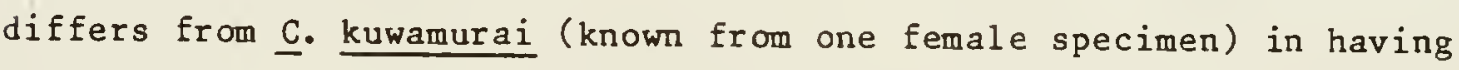
the dorsal fin entire (versus notched over dorsal spine XII); supraorbital cirri 2-7 (versus 12); nasal cirri 4-6 (versus 10); and small dark spots posteriorly on body (versus narrow pale stripes, red in life, on body).

Distribution. As presently recognized (see comments), Cirripectes auritus is known to occur in the Line Islands, Philippine Islands, at Similan Island, and in the western Indian Ocean from Kenya to South Africa (Figure 20). Although $\underline{C}$. auritus has been collected as deep as $20 \mathrm{~m}$ in the Comoros Islands (Carlson, 1981), it is usually found on coral reefs at depths less than about $10 \mathrm{~m}$. Specimens bearing the catalog numbers USNM 222489 and ANSP 138366 were collected at Fanning Island and have the same data as the holotype; Carlson (1981) erroneously reported them as being from Kenya. 
Etymology. The specific epithet is from the Latin auritus, meaning eared, and refers to the enlarged nuchal flaps (Carlson, 1981 ).

Comments. The meristic and morphometric characters of Cirripectes auritus do not vary geographically, but the color pattern does. Both sexes at Fanning Island have a mottled pigmentation pattern on the body, but, in the western Indian Ocean, only females have this pattern. No males are available from Similan Island or the Philippines, but Similan Island females resemble Fanning Island specimens, whereas the single Philippine female available differs in having an almost uniformly dark-brown backgiuund body color. Two South African males have the dorsalmost nuchal cirri somewhat darker than specimens from other areas. It is possible that these color pattern differences are indicative of the presence of more than one species, but there are too few specimens available to decide this with confidence.

There are some differences between my counts and those of Carlson (1981) for cartain characters based on the same specimens. Carlson (1981) stated that Cirripectes auritus has three segmented pelvic-fin rays, but I found four on al1 specimens I examined. It is probable that Carlson overlooked the small fourth ray, as it is only about half the length of, and closely applied to, the third ray. Carlson included only the independently based cirri between the nuchal flaps in his nuchai cirri counts, but I also included the smaller cirri borne on the nuchal flaps. Although the total number of centra in Carlson's vertebral counts on the two specimens in USNM 222489 are correct, they are incorrectly allocated to precaudal and caudal vertebrae. I found 
that the male has $10+22$ (not $9+23$ ) and the female has several aberrant anterior vertebral centra resulting in a count of $11+21$ (not $10+22$; I did not use this count to establish the range of variation for this character). In addition, I found that the same male has the last pleural ribs on centrum 11 (not 9), and the female appears to have pleural ribs on centrum 12 (the last ribs of the female could be on centrum 11, as it is difficult to tell on the radiograph). Based on a radiograph of both specimens in CAS 34383, I found that both specimens have small pleural ribs on centrum 11. Carlson (1981) lists one of the specimens as having the last pleural ribs on centrum 10, but the ribs are small and could easily have been overlooked.

Material examined. The following are paratypes of Cirripectes auritus: ANSP 138366 (2 specimens: $43,48 \mathrm{~mm}$ SL), Line Islands, Fanning Island, English Harbor near village, 5 m, 5 oct. 1975 (the locality of this lot was erroneously cited by Carlson, 1981); CAS 34383 (2), Grand Comoro Island, north of Hahaia, $20 \mathrm{~m}, 23 \mathrm{Feb}$. 1975; CAS $4894^{\circ}$ ( $1: 28$ ), Line Islands, Christmas Island, London, $01^{\circ} 58^{\prime} 41^{\prime \prime} \mathrm{N}$, $157^{\circ} 27^{\prime} 57^{\prime \prime} \mathrm{W}, 24 \mathrm{Aug}$. 1951; USNM 22489 (2: 50, 55), Line Islands, in English Harbor near village, $5 \mathrm{~m}, 5$ oct. 1975; USNM 222490 (1: 32), Philippine Islands, Apo Island, $09^{\circ} 04^{\prime} 30^{\prime \prime} \mathrm{N}, 123^{\circ} 16^{\prime} 24^{\prime \prime} \mathrm{E}$, $0-2.4$ m, 18 May 1979.

Other material examined. South Africa: RUSI 8888 ( $1: 38$ ), RUSI $9547(1: 48)$. 


\section{Cirripectes castaneus (Valeaciennes)}

Salarias castaneus Valenciennes in Cuvier and Valenciennes, 1836:324 (Isle-de-France [=Mauritius]).

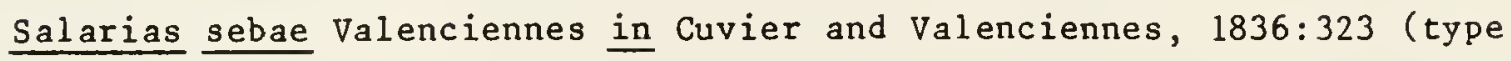
locality unknown).

Cirripectes reticulatus Fowler, 1946:173 (Aguni Shima, Ryu Kyu Islands).

Cirripectes gibbifrons Smith, 1947:815 (Inhaca Island).

Diagnosis. A species of Cirripectes with the following combination of characters: simple cephalic sensory pore pattern, one sensory pore posterior to ventral part of each nuchal flap, type I male genital papilla, bars or spots on body, 14 segmented dorsal-fin rays, 15 anal-fin rays, and 20 caudal vertebrae.

Description. Dorsal fin XI-XIII (XII in $99.5 \%$ of specimens),13-15 (14 in 95.2\%); anal fin II, 14-16 (15 in 93.4\%); total procurrent caudal-fin rays 10-14; pelvic fin 1,4 ; vertebrae 10-11 (11 in 99.8\%) + $19-21(20$ in $97.0 \%)=29-31$ (30 in $97.0 \%)$; last pleural ribs on vertebral centrum $11-12$ ( 11 in $99.8 \%$ ); last epipleural rib on vertebral centrum 17-21 (18-20 in 91.5\%); anal pterygiophores 1-1-1, 1-1-2, or $1-2-1$ (1-i-2 in 88.2\%); nuchal cirri 29-44 (32-40 in 95.8\%); supraorbital cirri 6-49 (6-20 in 94.8\%); nasal cirri 6-49 (8-20 in $92.3 \%)$; LL tubes $1-13$ ( $3-12$ in $94.3 \%)$; last LL tube positioned at point between verticals from dorsal-fin ray 5 and caudal-fin base ( 6 to caudal-fin base in 97.7\%); no scalelike flaps along LL; lower lip 
smooth mesially (plicate laterally); gill rakers 20-32 (based on 179 specimens); pseudobranchial filaments on one side 7-12 (based on 181 specimens); premaxillary teeth 178-222 (based on 33 specimens); dentary teeth 87-114 (based on 33 specimens); upper lip crenulae approximately 29-50; nuchal cirri in four groups with ventralmost group of cirri on each side borne on a slightly expanded nuchal flap (type B; Figure 7); first dorsal-fin spine of adults slightly longer than second (1.0-11.0 mm longer in males, $0-2.5$ mm longer in females); dorsal-fin membrane deeply incised above last dorsal-fin spine; dorsal-fin membrane attached to caudal fill in adults; cephalic pore system simple (1-2 pores at most positions); mid-snout pores present (Figure 17); extra interorbital pore position absent (Figure 17); pore positions behind nuchal flap 1 or 2 ( 1 pore on at least one side in 92.6\%; Figure 17); male genital papilla with urogenital orifice located basally between two widely separated slender filaments (less than $1.0 \mathrm{~mm}$ long) on a fleshy swelling behind anus (type I, Figure 13); testes bulbous, length equals width; maximum SL about $92 \mathrm{~mm}$; ophioblennius larvae with 2 canines posteriorly on each dentary.

The smallest mature female (ova about 0.6 mm diameter) examined was a Red Sea specimen about $36 \mathrm{~mm}$ SL. Males mature by about $35 \mathrm{~mm}$ SL. Color in alcohol. Coloration of ophioblennius stage larvae (based on 19-20 m SL Red Sea specimens) varies from uniformly pale brown to alternating pale and brown bars on head and body; all with about four alternating pale and brown diagonal bars on each side of snout extending from margins of orbit onto upper lip; fins transluscent; cirri brown. 
Juveniles (18-35 mm SL) of both sexes with brown head and body and about 3-10 dark-brown bars laterally on sides; nuchal and supraorbital cirri dark brown.

Males (Figure 30; Smith and Smith, 1963:pl. 37, fig. A, incorrectly identified as $\underline{\text { c. variolosus) }}$ larger than about $35 \mathrm{~mm}$ SL with alternating dark-brown and brown bars on head (about 4 dark bars) and body (about 10-11 dark bars), bars becoming fainter and background color darker near posterior end of body; bars on head extending from nape anteroventrally to underside of head, where they meet bars from other side of nead, forming 3 dark bars across gular region; dorsal fin dark brown with transluscent triangular area at tips of anterior spines; upper quarter to half of caudal-fin rays with pale triangular area on distal half of fin, remainder dark brown; anal fin dark brown with distal tips of rays pale; anal fin rugosities cream to brown; pectoral and pelvic fins dusky; nuchal cirri dark brown, other cirri brown.

Females (Figures 31 and 32) larger than about $40 \mathrm{~mm}$ SL with three types of color pattern: uniformly chocolate brown, barred (similar to adult males), and reticulated. I have not seen more than two pattern types in specimens from a given locality. South African females are either uniformly brown or barred. Kenyan females have a very dark-brown head and body; some specimens have dark-brown spots on the brown background of the head. Most females in most other populations have a reticulated pattern of pale pupil-sized spots and wavy brown lines, but there are usually a few females with a uniformly brown head and body. Generally, the reticulated pattern becomes less distinct 
posteriorly on body as spots become darker and farther apart and background color gets darker. Females usually have a pale bar on upper lip beneath each pupil. Other pigmentation patterns resemble those of males except the bars on throat may not be as distinct.

Color in life. Life colors of barred and uniform "brown" patterns are not known. Females with the reticulated pattern (based on color slides of specimens from the Red Sea, Chagos Archipelago, and Fiji; and Randal1, 1982b: fig. 273) have heads with pale greyish-brown spots separated by narrow brown (in Red Sea and at Chagos) or reddish-brown (at Fiji) wavy lines; anterior half of body reticulated or with narrow brown bars and greyish-brown spots, which sometimes coalesce into bars; posterior half of body with greyish-brown spots on dark-brown background that gradually darkens posteriorly to caudal-fin base; narrow brown lines extending from body onto lower half of dorsal-fin membrane between each pair of elements; orange to reddish-orange dorsal-fin spines extending through transluscent triangular area over distal part of spinous-dorsal fin; tips of segmented dorsal-fin rays orange to reddish orange; upper caudal-fin rays bright yellow (Red Sea and Chagos) or reddish orange ( $F i j i)$, lower rays yellowish brown or reddish brown, respectively; tips of ventralmost pectoral-fin rays orange to reddish orange; iris with irregular red ring around outer portion of eye, narrow yellow ring around pupil, and black area between rings (in some specimens yellow replaces black area); other colors as in alcohol.

Males have head and body (based on color slides of specimens from the Red Sea, Chagos Archipelago, and Fiji; see comments section for 
discussion of goographic variation) with alternating dark-brown and paler bars, pale bars varying in color (red, rusty brown, grey, or yellow); tips of dorsal-fin elements and upper caudal-fin rays varying from bright yellow to red; colors of other fins and iris as described for females.

Comparisons. Investigators frequently have confused Cirripectes

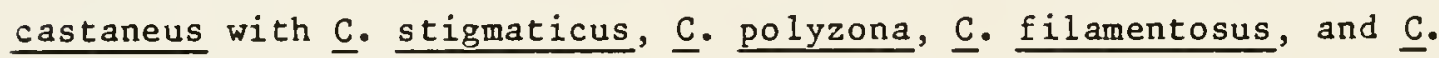
imitator in areas where these species occur sympatrically. All these species have similar male and/or female color patterns. Cirripectes

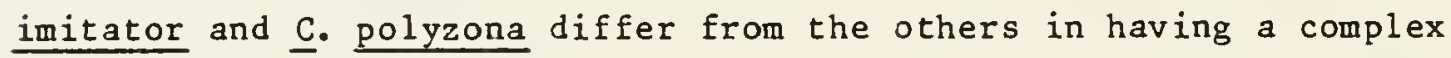
cephalic sensory pore pattern. Of the remaining three species, $\underline{C}$.

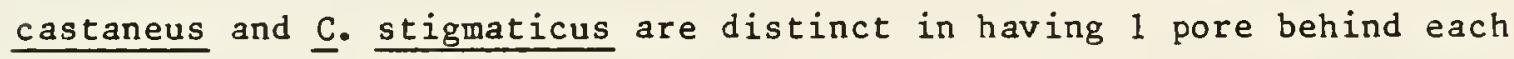
nuchal flap (versus 2 in $\underline{C}$. filamentosus), a type I (versus type II) male genital papilla, and bars or spots on body (versus uniformly brown

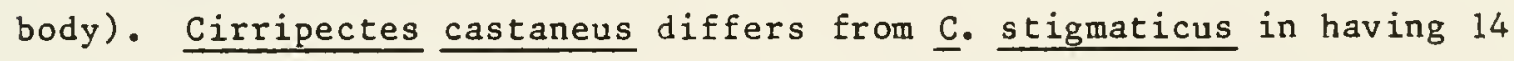
segmented dorsal-fin rays (versus 15), 15 anal-fin rays (versus 16), and 20 caudal vertebrae (versus 21 ).

\section{Distribution. Cirripectes castaneus is a common and widely} distributed species. It occurs throughout the Red Sea, Indian Ocean, and western Pacific to the margin of the Pacific plate (sensu Springer, 1982; Figure 21). Its only known occurrence on the Pacific plate is in the Caroline Islands. Although usually found on rocky and coralline substrates at depths less than $10 \mathrm{~m}$, it has been collected as deep as 30 m in the Red Sea and 32 m at Rowley Shoals, off Western Australia.

Etymology. The specific epithet is from the Latin castaneus, meaning brown, or of the color of chestnuts, and presumably refers to the color of the holocype in alcohol. 
Comments. Although I found no geographic variation in the morphometric characters examined, life colors of male Cirripectes castaneus vary geographically. In the Red Sea, some young males (:0 about 40 SL) have relatively narrow red bars on a brown background on head and anterior part of body. In the Chagos Archipelago, adult males have alternating brown and dark-brown bars, brillant yellow-orange dorsal-fin spine tips, and bright yellow upper caudal-fin rays. Both Red Sea and Chagos males have a very dark-brown background color of the head and body.

In Fiji, a nearly opposite life color pattern is found in Cirripectes castaneus males, which have brown bars on a pale grey background (versus brown bars on a dark-brown background). In a second color morph occurring in Fiji, background color of the head and body is yellow. As these are the only characters known to differ among these populations, and the life colors found in intermediate populations are as yet unknown, I do not believe these populations warrant taxonomic recognition.

The geographic ranges of $\underline{C}$. castaneus and $\underline{C}$. variolosus overlap only in the Caroline Islands and the northernmost Tonga Islands, where they are easily distinguished by cephalic sensory pore pattern, which is complex in $\underline{\text { C. variolosus. }}$

Nomenclatural discussion. Cirripectes castaneus frequently has been synonymized erroneously with $\underline{\mathrm{C}}$. variolosus (eg. Chapman, 1951; Herre, 1953; Lotan, 1970; and Smith, 1959).

My examination of the type specimens of Salarias sebae and $\underline{S}$. castaneus reveaied them to be conspecific. These two species were 
described on adjacent pages (․․ sebae on page 323 and $\underline{\text { S. }}$ castaneus on 324) by Valenciennes in Cuvier and Valenciennes (1836), which raises the question of which name should be used. Fukao (1984), as the first reviser to synonymize these names with each other, has clearly established $\underline{\text { S. }}$ castaneus as a senior synonym to $\underline{\text { S. }} \underline{\text { sebae }}$ (no previous reviser recognized the conspecificity of the types of these species). Prior to Fukao, as a result of the work of Chapman (1951) and Schultz and Chapman (1960), Cirripectes sebae was applied most frequently to specimens of the unrelated $\underline{C}$. polyzona. Chapman's (1951) description of $\underline{C}$. sebae was based on a conglomerate of two or more species. Of the three color-pattern types he described, the first two apply to female C. polyzona, and the third could apply to female $\underline{C}$. castaneus $(=\underline{C}$. sebae), female $\underline{\text { C. stigmaticus, }}$ or either sex of $\underline{C}$. imitator. Schultz and Chapman (1960) appear to have applied the name $\underline{C}$. sebae only to specimens of $\underline{C}$. polyzona, as the other similar species (except $\underline{C}$. stigmaticus) do not occur in the Marshall Islands. Bleeker, very early, correctly applied the names $\underline{\mathrm{C}}$. sebae and $\underline{\mathrm{C}}$. polyzona, but his findings were published only recently (Bleeker, 1983, pI. 4). Randall

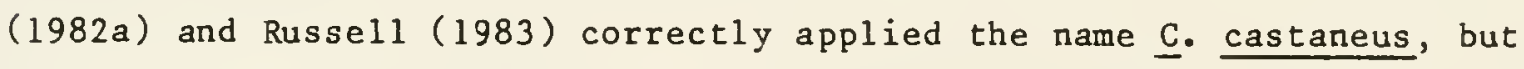
made no mention of the name $\underline{\mathrm{C}}$. sebae.

The descriptions of Cirripectes reticulatus Fowler (1946) and Cirripectus gibbifrons Smith (1947) are based on the reticulated color morph females of Cirripectes castaneus. Although Fukas (1984) correctly recognized C. castaneus as a valid name, he erroneously synonymized $\underline{\mathrm{C}}$. reticulatus and $\underline{\mathrm{C}}$. gibbifrons with $\underline{\mathrm{C}}$. polyzona (Williams, 1985). 
Material examined. Types: MNHN A.1799 ( 1 specimen: $42 \mathrm{~mm}$ SL), holotype of Salarias castaneus, Isle de France, collection date unknown; MNHN A.2059 (1: 43), holotype of Salarias sebae, from the "Cabinet du Stathouder," locality and date of collection unknown; ANSP 72043 (1: 67), holotype of Cirripectes reticulatus, Riu Kiu Islands, Aguni Shima, 27 July 1945; RUSI 245 (1: 49), holotype of Cirripectus gibbifrons, Mozambique, Inhaca Island, collection date unknown.

Other material examined. South Africa: RUSI UNCAT. (1), RUSI 75-5 (1: 54), RUSI 75-8 (1: 23), RUSI 75-9 (6:37-55), RUSI 76-8 (5), RUSI 76-10 (5), RUSI 76-11 (11), RUSI 76-12 (1), RUSI 76-16 (3), RUSI 76-18 (1), RUSI 76-21 (20: 42-72), RUSI 76-23 (1), RUSI 76-28 (2), RUSI 77-16 (7), RUSI 77-17 (3: 61-82), RUSI 77-18(1: 76), RUSI 9477 (1), RUSI 9824 (6), RUSI 9941 (3), RUSI 10024 (5), US NM 227345 (10). Mozambique: RUSI 14998 (10), RUSI 15002 (3: 36-59), RUSI 15011 (11). Tanzania: RUSI 14997 (8: 21-40), USNM 228219 (20 of 58: 52-79). Kenya: RUSI 15009 (5), RUSI 15012 (6), UF 34643(1:40), USNM 228278 (1: 47), US NM $228282(1: 63)$. Aden: US NM 228216 (4:21-52). Saudi Arabia, Red Sea: USNM $147584(1: 26)$, USNM 147586 (3: 34-44). Sudan: BMNH 1960.2.15.1589 (1), BPBM $19719(14: 28-46)$, NMW 73225 (6). Egypt: USNM 228185 (6: 49-59), USNM 228227 (2), USNM 228230（5: 27-39). Israel: BPBM 18332 (9), BPBM 19803 (6), USNM 228186 (1 of 22: 20), USNM 228187 ( 1 of 16: 19), USNM 228194 (1:38), USNM 228195 (12), USNM $228196(21: 19-63)$, USNM 228198 ( $8: 19-42)$, USNM 228200 (11: 19-31), USNM 228204 (54 of $94: 19-58$ ), USNM 228223 ( $1: 60$ ), USNM 228229 (4). Madagascar: MNHN uncataloged (numerous specimens collected by A. Mauge), USNM 228225 (2), USNM 228327 ( 1 ). Comoros Islands: CAS 32548 
(1: 49), CAS 40376 (1), CAS 40378 (14), CAS 40382 (16), USNM 228173 (49: 24-64), USNM 228215 (2), USNM 228249 (8). Seychelles Islands, Farquhar Group: USNM 228177 (29: 31, 59), USNM 228183 (19 of 20 examnined: 25-60). Seychelles Islands, Amirantes Group: ANSP 126861 $(2: 51,62), \operatorname{ANSP} 126862(2: 28,64)$, ANSP $126983(9: 27-59)$, ANSP 127003 (1: 33). Seychelles Islands, Cosmoledo Group: ANSP 147666 (2: $31,71)$, ANSP $147668(3: 62-66)$, ANSP 147669 ( $1: 60)$, ANSP 147670 (2: 26,32 ), BMNH 1908.3.23.277 (1), BMNH 1969.1.24.47-73 (27), RUS I 14993 (4), USNM 228164 (2), USNM 228167 (20), USNM 228168 (16), USNM 228169 (1), USNM 228170 (19), USNM 228174 (2), USNM 227175 (4), USNM 228188 (1). Agelaga Islands: USNM 228207 (3), USNM 228211 (1), USNM 228212 (21: 37-54), USNM 228242 (1), USNM 228250 (5). Cargados Carajos Shoals: USNM 228244 (2 of 3 examined: 78, 82). Mauritius: BPBM 20206 (2), ВPBM 21021 ( $1: 58)$, USNM 228179 (2:38, 55). Reunion Is land: BPBM 20033 (2: 52-61). Chagos Archipelago: ROM 46601 (1: 48), ROM 46602 (1: 42), ROM 46603 (5: 26-63), ROM 46604 (1:38), ROM 46605 (4: 40-64), ROM $46606(4: 33-62)$, ROM $46607(1: 36)$, USNM 228176 (2), USNM $228180(22: 36-77)$, USNM $228181(3: 39-62)$, USNM $228184(1: 47)$, USNM 228190 (6: 54-77), USNM 228214 (12), USNM 228218 (16), USNM 228243 (14). Cocos-Reeling Islands: ANSP 134750 (65 of 112 examined: 25-75), ANSP 134751 (49: 25-65), ANSP 134752 (57 of 75 examined: 23-59), ANSP 134753 (4: 32-59), ANSP 134754 (2: 34, 67), ANSP 134755 (14), ANSP 134756 ( $1: 37)$, ANSP 134757 (25: 23-57), ANSP 134758 (8: 39-70), ANSP 134760 ( $1: 35)$, ANSP 134761 (2: 27-35), ANSP 134762 (9:23-40), ANSP $134763(2: 30,34)$, ANSP $134764(5: 25-56)$. Christmas Island, Indian Ocean: WAM P.26110-033 (1: 38). Australia, Western Australia: AMS 
I.21318-072 (3: 44-51), WAM P.27667-032(2: 45, 51), WAM P.27659-011 (1: 61). India, Kerala State: ANSP 147657 (1: 29). Sri Lanka (Ceylon): USNM 228228 (1: 21), USNM $228247(1: 56)$. Thailand, Andaman Sea: BPBM $22785(1: 66)$, USNM 228221 (2: 40, 45). Vietnam: CAS UNCAT. (26 of 51 examined: 45-83). China: CAS 38846 (5: 56-66). Taiwan: USNM 228051 (1), USNM 228182 (1: 56), USNM 228224 (3: 31-69). Japan: ANSP 113692 (2: 76, 78), BPBM 7252 (7: 58-81), FAKU 111456 (1: 65), FAKU 48108 (2: 68, 73), FAKU $48271(1: 34), \operatorname{FAKU} 48926(1: 52)$, FAKU $48947(1: 25)$, FAKU $48965(1: 25)$, FAKU $48973(1: 31)$, FAKU 49173 (2: 47-61), FAKU 49175(1: 66), FAKU 49176(1: 71), FAKU 49177(2: 71: 71), FAKU 49178 (1: 70), FAKU $49179(2: 57,72)$, FAKU 49182 (2: 89, 92), FAKU 50401 (1: 52). Philippine Islands: LACM UNCAT. (1), LACM 4279-19 (2), USNM 224951 (2), USNM 227552 (2), USNM 227554 (1), USNM 228062 (2), USNM 228171 (1), USNM 228191 (2: 47, 58), USNM 228192 (3), USNM $228193(23: 31-55)$, USNM $228197(6: 8-70)$, USNM 228199 (7), USNM 228202 ( $2: 77,78$ ), USNM 228208 (3), USNM 228210 (1), USNM 229252 (2), US NM 228281 (1: 45). Indonesia: AMS J.19875-091 (13:44-52), RMNH 12639 (1), RMNH 20605 (2), US NM 209561 (3), USNM 228172 (11: 19-40), US NM $228203(2: 38,45)$, USNM 228206 (3), USNM $228222(3: 44-55)$, USNM 229246 (1), USNM 228279 (1: 75). Papud-New Guinea: AMS I.17094-074 (4: 27-60), USNM 212136 (1), USNM 228201 (8: 22-55), USNM 228213 (1: 65), USMM 228240 (4). Australia, Queensland: AMS I.15638-048 (1), AMS I. 18740-014 (1), AMS I.18740-015 (1), AMS I.19473-204 (24), AMS I.19481-015 (2), AMS I.20467-005 (1), AMS I.20770-098 (8), AMS I.20774-077 (1), AMS I.20912-002 (2), AMS I.21422-046 (3), AMS 
I.15621-022 (1), AMS I.15646-012 (1), AMS I.17112-004 (1), AMS

I.17445-011 (2), AMS I.17445-042 (3), AMS I.19483-033 (35: 53-87), AMS I.20756-083 (2: 43, 46). AN3P 109730 (3: 31-46), ANSP 147658 (1: 65), USNM 228165 (5: 50-75), USNM 228205 (56), USNM 228217 (5), USNM 228220 (1), USNM 229245 (1). Lord Howe Island: AMS I.17368-035 (1: 59). Norfolk Island: AMS I.20268-037 (1: 41). Solomon Islands: CAS UNCAT. (1: 47), CAS UNCAT. (1: 42). Vanuatu (previously New Hebrides): BPBM UNCAT. (4), BPBM 5708 ( $1: 55)$. Loyalty Islands: USNM UNCAT. (1: 20; OUT OF 228274), USNM $228166(1: 71)$, USNM $228178(1: 76)$. New Caledonia: MNHN 1980.959 (2). Fiji Islands: BMNH 1877.4.26.! (1), BMNH 1877.12.10.63 (1), BMNH 1897.8.23.215-217 (3), BPBM 14629 (4), USNM 259996 ( 3 ), USNM 227209 (1), USNM 228189 (32: 39-77), USNM 228241 (1), USNM 235726 (52), USNM 235729 (9), USNM 235730 (4), USNM 235733 (1), USNM 235734 (3). Tonga Islands: USNM 228248 ( $1: 56$ ), USNM 228251 (1: 64). Caroline Islands: CAS UNCAT. (2: 40, 50), CAS UNCAT. (1: 46), CAS UNCAT. (6:51-65), CAS 38841 (1: 38), CAS $38842(1: 43)$, CAS 48894 (1), CAS 48907 (1: 40), CAS 48910 (4:23-57), CAS 48925 (1), CAS $48927(1: 60), \operatorname{CAS} 48944$ (2), $\operatorname{CAS} 48951$ (2), CAS 48968 (2: 38, 44), CAS 48969 ( $1: 58)$, CAS 49901 (1).

\section{Cirripectes chelomatus Willams and Mauge}

Cirripectes chelomatus Ni11iams and Mauge, 1983:1140 (Lady Musgrave Reef, Queensland, Australia).

Cirripectes sp. Russell, 1983:114 (Capricorn-Bunker Group of Islands, Queensland, Australia). 
Diagnosis. A species of Cirripectes with the following combination of characters: incnmplete lateral line, type II male genital papilla (Figure 13), dorsal fin entire, I, 4 pelvic-fin rays, 15 segmented dorsal-fin rays, 16 segmented anal-fin rays, and 30 caudal vertebrae.

Description. Dorsal fin XII-XIII (XII in $98.6 \%$ of specimens), 14-16 ( 15 in $91.6 \%)$; anal fin II, 15-17 (16 in $89.9 \%)$; total procurrent caudal-fin rays 10-14; pelvic fin $I, 4$; vertebrae $9-11$ (10 in $97.8 \%)+$ $19-21(20$ in $94.8 \%)=29-31(30$ in $96.4 \%)$; last pleural ribs on vertebral centrum 11; last epipleural rib on vertebral sentrum 15-18 (15-17 in $97.7 \%)$; anal pterygiophores $1-1-2,1-2-1$, or $2-1-1(1-2-1$ in 74.8\%); nuchal cirri 22-45 (27-32 in $80.6 \%$ ); supraorbital cirri 3-93 (6-9 in $82.1 \%$; one specimen each with 23 and 93 , al1 others with 12 or fewer); nasal cirri 6-82 (6-15 in 83.5\%; one specimen with 82 , all others with 25 or fewer); LL tubes $0-8(1-5$ in $89.6 \%)$; last LL tube or end of LL canal (if no tubes) positioned at point between verticals from dorsal-fin spine XII and dorsal-fin ray 10 (rays $3-7$ in $89.0 \%$; one specimen with a widely disjunct tube beneath ray 15); no scalelike flaps along LL; lower lip smooth mesially (plicate laterally); gill rakers 20-28 (based on 85 specimens); pseudobranchial filaments on one side 6-1C (based on 85 specimens); premaxillary teeth 200-231 (based on 18 specirens); dentary teeth 93-120 (based on 19 specimens); upper lip 
crenulae approximately 29-43; nuchal cirri in four groups (some cirri in dorsalmost groups sometimes not connected at their bases) with a slightly expanded flap at bases of ventralmost cirri (type B; Figure 7); first dorsal-fin spine of adult males 2-19 mm longer than second, first dorsal-fin spine of adult females 0-9 mn longer than second; dorsal-fin membrane with very shallow notch above last dorsal-fin spine; dorsal-fin membrane attached to caudal fin in adults; cephalic pore system simple (less than 3 pores at most positions; number of pores increases with increasing SL); mid-snout püres frequently absent (Figure 17); extra interorbital pore position ahsent (Figure 17); two pore positions behind nuchal flap (Figure 17); male genital papilla with urogenital orifice located basally between two closely appressed slender filaments (less than 1.0 m long) on a fleshy swelling behind anus (type II; Figure 13); testes bulbous, length equals width; maximum SL about $94 \mathrm{~mm}$ ophioblennius stage larvae of this species have not been examined.

The smallest mature female (ova about 0.5 m diameter) examined was about 40 SL. Males mature at about $35 \mathrm{~mm}$ SL.

Color in alcohol. Adult males and females have similar color patterns. Head brown with diagonal streak of tiny, sharply defined, cieam-colored spots extending from postcrbital area to dorsal edge of upper lip; nasal and supraorbital cirri brown or tan; nuchal cirri dark brown or black. Body brown with cream-colored spots dispersed over middle third of body, dorsalmost spots on basal third of dorsal fin, ventralmost near anus and on membranes of lower third of anal fin, spots gradually darken posteriorly. Dorsal fin with transluscent tips, 
remainder dusky. Other fins dusky. Anal-fin spine rugosities of males pale. Additional details given by Williams and Mauge (1983).

C.slor in life. As described by Williams and Mauge (1983), an adult male from One Tree Island, Australia, with red spots on head; nasal and supraorbital cirri yellowish; nuchal cirri black basally and at tips, with yellow band around middle; iris red; purplish-red spots on middle of body, spots gradually darken to dark brown posteriorly; pectoral fin yellowish; dorsal-fin elements with orange-yellow tips, spots on basal portion of fin purplish red; spots on andi fin purplish red; anal-spine rugosities dirty ye11ow.

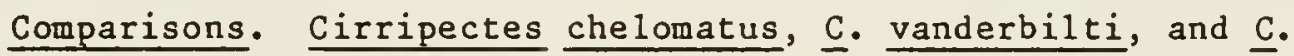
filamentosus are the only Cirripectes species with a type II genital papilla. Characters differentiating $\underline{C}$. chelomatus and $\underline{C}$. filamentosus are discussed in the account of the latter. Cirripectes vanderbilti only occurs in the Hawaiian Islands and at Johnston Island, and has a type $G$ nuchal cirri arrangement (Figure 7 ).

Cirripectes chelomatus (Figure 33) and $\underline{\text { C. springeri (Figure 58) }}$ have similar color patterns, but differ in that $\underline{\underline{c}}$. chelomatus has a type II male genital papilla (versus type I), anal-fin pterygiophores typicaliy 1-2-1 (versus 1-1-2), and 30 vertebrae (versus 31 ).

Distribution. Cirripectes chelomatus is known to occur on coral reefs and rocky bottoms off eastern Australia from Lady Musgrave Reef r.crthwaid to Sir Charles Hardy Island; as far north as Basilaki Island, Papua-New Guinea; as far south as Lord Howe Island; and eastward to the Fiji and Tonga Islands (Figure 26). It is represented in a collection of fishes made at depths of $0-16$ m in the Tonga Islands, but most specimens were collected in water shallower than $7 \mathrm{~m}$. 
Etymology. The specific epithet is derived from the Greek cheloma, meaning notch, and refers to the shallow notch in the dorsal-fin membrane above the last dorsal spine. The name is used as a noun in apposition.

Comments. As discussed by Williams and Mauge (1983), geographic variation occurs in color pattern. The few available Fiji specimens have poorly defined spots on the body. Additional material needs to be examined to determine the extent of this variation.

Material examined. A complete listing of localities for the macerial examined is given in Williams and Mauge (1983). AMS I.21495-027 (male: 66 m SL), holotype of Cirripectes chelomatus, Lady Musgrave Reef, Queensland, Australia, $23^{\circ} 55^{\prime} \mathrm{S}, 152^{\circ} 24^{\prime} \mathrm{E}, 20 \mathrm{Feb}$. 1980 .

The following specimens are paratypes. Australia, Queensland: AMS I.15482-011 (2), AMS I.15634-029 (1), AMS I.17109-015 (1), AMS I.17362-037 (1), AMS I.17445-150 (1), AMS I.19338-008 (4), AMS I.19444-036 (1), AMS I.19473-205 (13), AMS I. 20205-005 (3), AMS I.20210-016 (1), AMS I.20213-007 (1), AMS I.20464-005 (2), AMS I. 20560-014 (1), AMS I.20770-071 (4), AMS I.21490-032 (1), ANSP 109717 (6), ANSP 147659 (1), BMNH 1982.11.3.1 (1), BPBM 14449 (2), ВPBM 15024 (1), BPBM 28196 (1), BPBM 28197 (2), CAS 13832 (10), LACM 33732-20 (1), ROM 40368 (2), UF 35398 (2), USNM 228298 (3), USNM 228299 (4), USNM 228300 (1), USNM 229301 (5), USNM 228302 (11), USNM 228303 (4), USNM 228305 (2), USNM 228306 (3), USNM 228308 (9), USNM 228309 (2), USNM 228310 (44), WAM P.27754-001 (2). Lord Howe Island: AMS I.17362-037 (1). New Caledonia: CAS 48957 (1), CAS 48958 (1), MNHN 1983-257 (1), 
USNM 195783 (1). Loyalty Islands: USNM 228274 (3), USNM 228304 (3). Tonga Islands: USNM 228291 (4). Vanuatu (=New Hebrides): CAS 48903

(1). Papua-New Guinea: USNM 228307 (1).

Other material examined. Fiji Islands: ROM 40207 (3), USNM 228277 (1), USNM 235727 (2), USNM 235731 (2), USNM 235735 (2).

\section{Cirripectes filamentosus (Alleyne and Macleay)}

Salarias filamentosus Alleyne and Macleay, 1877:337 (Cape York). Salarias cruentipinnis Day, 1888:797 (Saddle Island, off Kyoukphoo in Arracan).

Cirripectes indrambaryae Smith, 1934:322 (Koh Samui, Gulf of Siam).

Diagnosis. A species of Cirripectes with the following combination of characters: uniformly brown body, small pale spots (red in life) on cheeks and snout, last lateral-line tube usually positioned at point between verticals from dorsal-fin ray 2-6, 2 pores behind nuchal flap, and 30 total vertebrae.

Description. Dorsal fin XI-XIII (XII in $95.8 \%$ of specimens),13-16 ( 14 in $40.8 \%, 15$ in $57.7 \%$ ); anal $\mathrm{Ein}$ II, $14-17$ ( 15 in $39.0 \%, 16$ in $60.1 \%)$; total procurrent caudal-fin rays $8-14(10-12$ in $95.7 \%)$; pelvic fin $1,3-4$ (geographic variation discussed in comments section); vertebrae 10 (one specimen with 11 ) $+19-21(20$ in $91.1 \%$ ) = 29-31 ijo in $91.9 \%)$; last pieural ribs on vertebral centrum 11; last epipleural rib on vertebral centrum 13-17 (14-16 in 92.5\%); anal pterygiophores $1-1-1,1-1-2,1-2-1$, or $2-2-1 \quad(1-2-1$ or $2-1-1$ in $87.2 \%)$; nuchal cirri 
22-39 (25-31 in $82.6 \%)$; supraorbital cirri 4-13 (4-10 in $94.6 \%)$; nasal cirri $4-34(6-14$ in $83.8 \%)$; LL tubes $0-8(0-5$ in $98.5 \%)$; last LL tube or end of LL canal (if no tubes) positioned at point between verticals from dorsal-fin spine XII and dorsal-fin ray 11 (rays 2-6 in 97.1\%); no scalelike flaps along LL; lower lip smooth mesially (plicate laterally); gill rakers 21-27 (based on 140 specimens); pseudobranchial filaments on one side 6-9 (based on 141 specimens); premaxillary teeth 151-189 (based on 17 specimens); dentary teeth 72-103 (based on 17 specimens); upper lip crenulae approximately 26-50; nuchal cirri in four groups with cirri in dorsalmost groups sometimes not connected at their bases, cirri in ventralmost groups borne on a slightly expanded nuchal flap (type B; Figure 7); most dorsal-fin spines of adults filamentous, the first dorsal-fin spine longer than second $(1.0-9.6 \mathrm{~mm}$ longer in males, 1.0-6.0 mn longer in females); dorsal-fin membrane distinctly incised above last dorsal-fin spine; dorsal-fin membrane attached to caudal fin in adults; cephalic pore system simple (less than 3 pores at most positions; Figure 17); mid-snout pores presert or absent (varies geographically); extra interorbital pore position absent (Figure 17); two pore positions behind nuchal flap (Figure 17); male genital papilla with urogenital orifice located basally between two closely-appressed, small, slender filaments (less than 1.0 mm long) on a fleshy swelling behind anus (type II; Figure 13); testes bulbous, length equals width; maximum SL about $70 \mathrm{~mm}$; ophioblennius iarvae examined nad no canines posteriorly on dentaries.

The smallest mature female (ova about $0.5 \mathrm{~mm}$ diameter) examined was about 39 mn SL. Females $35-36 \mathrm{~mm}$ SL were not gravid but had developing ova (0.1-i. 2 man diameter). Males mature by about 35 an SL. 
No sexual variation was found in any of the meristic characters examined.

Color in alcohol. Ophioblennius stage larvae (to about $20 \mathrm{~mm}$ SL) with head and body cream colored; no distinct blackish spots, but several areas around lower half of orbit with slightly denser grouping of small melanophores than surrounding areas; all cirri cream colored.

Some specimens about 25-35 mm SL with broad dark-brown stripe (its width about the diameter of orbit) on dorsal half of body beginning above pectoral-fin base and reaching almost to caudal-fin base; background color pale brown; others with a uniformly dark-brown body; frequently, a narrow pale streak, often broken into pale spots, extending diagonally from behind eye onto upper lip.

Adult males and Females (Figure 34) with dark-brown body. Some specimens with faint indication of above-mentioned broad dark stripe (stripe not contrasting with dark body); several pupil-sized pale-brown spots frequently present above pectoral-fin base; head brown with pale spots on cheeks and around eyes, often with pale streak extending from postorbital margin to upper lip; two faint pale bars (width of each about half pupil diameter) sometimes present under eye, one from posteroventral margin of orbit to point on upper lip beneatin middle of orbit, the other from anteroventral margin of orbit onto upper lip near symphys is .

Color in life. Adult male (based on a color sidue of a freshly deaủ specimen from Dampier Archipelago, Western Australia) with head and anterior fourth of body pale brown, posterior three-fourths of body dark brown; head with small red spots and streaks; bars beneath eye 
pale yellowish-brown; nasal cirri yellowish brown; orbital cirri pale brown; nuchal cirri blackish; pectoral-fin rays yellowish; dorsal-fin spines reddish, lower half of spinous-dorsal fin brown, segmented-dorsal fin dark brown with tips of anterior rays reddish; proximal half of caudal fin brown, distal half with upper rays yellow and lower rays reddish; anal fin with tips of rays whitish, then sequentially toward body a red stripe, brown stripe, red stripe, and brown stripe, each becoming darker posteriorly; iris with yellow inner ring and red outer ring; reddish pink rugosities on anal-fin spines.

A young female (based on a color slide of a Tioman Island, Malaysia specimen) has coloration similar to the male described, except the segmented-ray portion of the dorsal fin and the entire caudal fin are yellowish, the lower pectoral-fin rays are orangish red, and the red spots extend from the head onto the body above the pectoral-fin base.

Compariscns. Cirripectes filamentosus is most similar to $\underline{\mathrm{C}}$. chelomatus. These species occur sympatrically in northeastern Queensland and southeastern New Guinea, where they wan be differentiated by coloration and pelvic-fin ray counts. Cirripectes chelomatus has small brown (purplish-red in life) spots covering the body and I, 4 pelvic-fin rays. Cirripectes filamentosus lacks spots on the body and has I, 3 pelvic-fin rays in the area of sympatry (some populations in other geographic areas have I, 4 or both I, 3 and I,4). Male Cirripectes filamentosus are distinct from all sympatric

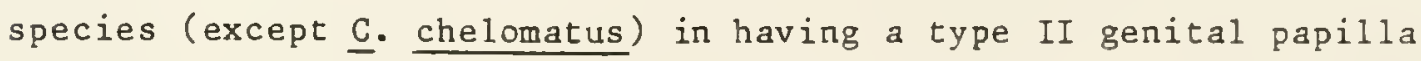
(Figure 13). Females and young are distinguished from sympatric species by the following combination of characteristics: uniformly 
brown body, small pale spots (red in life) on cheeks and snout, last lateral-line tube usually positioned at point between verticals from dorsal-fin ray $2-6,2$ pores behind nuchal flap, and 30 total vertebrae. Distribution. Cirripectes filamentosus occurs in the western Indian Ocean from northern Madagascar and Cargados Carajos Shoals northward to the southern Red Sea and the Persian Gulf. Although known from the Seychelles Islands, it has not been collected at Chagos Archipelago or the Cocos-Keeling Islands. Based on material I have examined, a disjunct population is known from the eastern Indian Ocean, where it occurs from Western Australia to the Andaman Sea, throughout the Indo-Australian Archipelago to the Solomon Islands, and as far north as Taiwan, but not on the Pacific plate.

The disjunct distribution in the Central Indian Ocean (Figure 19) is probably a collecting artifact. Jones and Kumaran (1980) reported specimens identified as Cirripectes variolosus from the Laccadive Islands, but, as this species does not occur in the Indian Ocean, the specimens may be $\underline{C}$. filamentosus. My attempts to borrow these specimens for examination have been unsuccessful. If these specimens are referable to $\underline{C}$. filamentosus, they fill part of the gap between the eastern and western Indian Ocean populations.

Specimens of Cirripectes filamentosus seem to be better colonizers or able to tolerate a wider range of environmental conditions than other Cirripectes species. Cirripectes filamentosus is the only member of the genus occurring in the Persian Gulf, Gulf of Thailand, and along the coast of Northern Territory, Australia.

Cirripectes filamentosus is usually found on coral or rocky reefs from shallow water to about $20 \mathrm{~m}$. 
Etymology. The specific epithet is derived from the Latin filum, meaning thread, and presumably refers to the filamentous dorsal-fin spines of adults.

Comments. Cirripectes filamentosus shows more variation in more characters than any other Cirripectes species. It is the only species that does not have a strong mode for dorsal- and ana1-fin segmented rays (i.e. in each population, almost half the specimens have 14 and half 15 dorsal rays, and about half have 15 and half 16 anal rays). All other Cirripectes species have a strong mode for a single count in each fin. The variation in number of segmented pelvic-fin rays in $\underline{\text {. }}$ $\underline{\text { filamentosus is particularly noteworthy. For every other Cirripectes }}$ species, this character is extremely constant. Cirripectes

filamentosus not only exhibits geographic variation in this count, but can have both counts (I,3 and $I, 4$ ) common in specimens from a single locality (e.g. of 37 specimens from the Philippines, 25 have I, 3 and 12 have I, 4; at Tioman Island 3 have I, 3 and 2 have I, 4; at each of these localities, one additional specimen has $I, 3$ on one side and $I, 4$ on the other). Specimens from most other localities have I, 3 pelvic-fin elements (one specimen in the western Indian Ocean has I, 3 on one side and I, 4 on the other), but specimens in the Gulf of Thailand and Viet Nam (one specimen examined) above the equator, and Northern Territory and Western Australia below the equator; all hav 1,4 (one Western Australian specimen with I, 3 on one side and I, 4 on the other). One other character, presence or absence of mid-snout pores, shows the same pattern of geographic variation. In those populations (localities) with both pelvic-fin ray formulae and those with only I,4, mid-snout 
pores may be present or absent. Those populations with I, 3 pelvic-fin rays have mid-snout pores present.

Although the variation discussed above suggests a divergence of certain populations from others, I do not believe these differences warrant recognition of these populations at the specific or subspecific level.

Another character showing a high degree of variation in Cirripectes filamentosus is the length and number of filamentous dorsal spines. The spines become filamentous with increasing SL, and are often best developed in adult males. However, some groups of similar-sized males and females may contain individuals of each sex with short filaments and others with very long filaments. The first spine is usually long and filamentous in adults of both sexes. Adult males usually have most of the spines filamentous, whereas adult females can have anywhere from none to almost all filamentous.

Nomenclatural discussion. Alleyne and Macleay (1877) described Salarias filamentosus based un one specimen from Cape York, Australia. They erroneously described the specimen as having XII,20 dorsal-fin elements, II, 20 anal-fin elements, and no canines. McCulloch and McNeil1 (1918) corrected the counts of Alleyne and Macleay (1877) to dorsal XII, 15 and anal II,16, and found a canine posteriorly on each dentary. My examination of the holotype (AMS I.16408-001) confirms the observations of McCulloch and McNeill (1918).

The description of Salarias cruentipinnis by Day (1888) was based on a description and illustration in a Tickell manuscript. The specimen on which the description was based is presumed lost. The 
illustration and description generally fit cirripectes filamentosus, but there are a few differences (based on my examination of a color photograph (slide) of the description and illustration in the Tickell manuscript). The illustration shows a continuous dorsal fin and a distinct yellow patch behind the posterior edge of the maxillary. I have not seen a Cirripectes specimen with similar yellow markings. Several species have a continuous dorsal $\mathrm{fin}$, and $\mathrm{I}$ have even seen $\underline{\mathrm{C}}$. filamentosus specimens without the notch (the result of damage and regeneration of the membrane, probably during early development). Aside from the yellow patches, Salarias cruentipinnis generally resembles $\underline{\mathrm{C}}$. filamentosus and I consider it a junior synonym of $\underline{\mathrm{C}}$. filamentosus. The type locality of Salarias cruentipinnis, Saddle Island, is the only Burma locality from which a Cirripectes has been recorded.

Cirripectes indrambaryae was described by Smith (1934), based on one specimen from Koh Samui, Gulf of Thailand. I have not examined the type, but, aside from the lack of canines in the lower jaw, the description and the illustration of the type (the latter published by

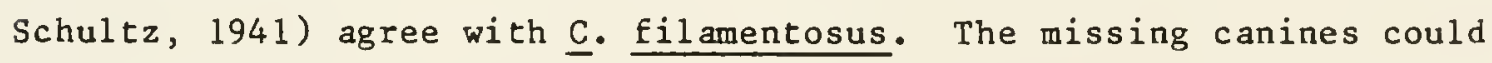
have been broken off (not an uncommon occurrence), or they may have been present and overlooked. In addition, C. filamentosus is the only Cirripectes species known to occur in the Gulf of Thailand. Consequently, I consider $\underline{C}$. indrambaryae to be a junior synonym of $\mathrm{C}$. filamentosus.

Material examined. Types: AMS I.16408-001 (55 SL), male holotype of Salarias filamentosus, Cape York, Queensland, Australia. 
Other material examined. Kenya: RUSI UNCAT. (2: 54-46). Ethiopia: HUJF 11241 (6: 34-53), HUJF 11242 (1: 26), USNM 204500 (4: 29-41), USNM 204512 (1: 38), USNM 204520 (9: 29-51), USNM 204521 (3: 31-37). Yemen: USNM 230382 (1: 28), USNM 230383 (1). Aden: USNM 228050 (1). Omman: BPBM 21378 (1: 48), BPBM 21464 (4: 39-56), ROM UNCAT. (3: 46-54), ROM UNCAT. ( 1 ; SIMM 82-14), ROM UNCAT. (2; SIMM 18-04). Saudia Arabia, Persian Gulf: BPBM 30281 (1). Madagascar: USNM 228057 (2: 40-46). Seychelles Islands, Farquhar Group: ANSP 114817 ( $3: 28-54)$, ANSP 126857 ( $1: 31)$, ANSP 126858 (1: 29), ANSP $126859(3: 29-36)$, ANSP 126860 (3: 28-31), AiviP 126871 (3: 31-43), ANSP 126936 ( $2: 23,44)$, ANSP 126937 (6: 32-57), ANSP 126984 (15: 24-55), ANSP 126985 (18: 23-46), ANSP 126986 (51: 16-51), ANSP 126987 (28: 16-52) ANSP $126988(114: 19-54)$, ANSP $127001(3: 33-37)$, ANSP 127002 $(2: 39,66)$, ANSP $127004(3: 32-38)$, ANSP $127005(2: 55,58)$, ANSP $127127(1: 17)$, ANSP $127139(10: 29-53)$, ANSP 127140 (12:18-59), ANSP 127141 (36: 18-53), ВРВM 21583 (22), ВРВM 22968 (6). Cargados Carajos Shoals: USNR: 228054 (4), USNM 228055 (6), US NM 228056 (6), USNM 228058 (34), USNM 228059 (1), USNM 228060 (22), USNM 228061 (1), USNM 228092 ( 30 of 53 examined: $34-60$ ), USNM 228100 (34), USNM 228104 (9), USNM $228156(1: 51)$, USNM 229157 (1: 62). Australia, Western Australia: AMS I. 19688-004 (2), BPBM 17427 (20:18-65), WAM P.22417-9 ( $1: 47)$, WAM P.27496-001 (2: 40-58). Nicobar Islands: $\operatorname{SMF} 17081$ ( $1: 35)$. Thailand, Similan Islands: BPBM 22798 (6: 35-51), USNM 228094 (1). Thailand, Gulf of Thailand: CAS 48887 (2), CAS 48888 (8), CAS $4888 \bar{y}$ (1), CAS 48890 (2), CAS 48904 (7), CAS 48913 (17), CAS 48914 (15), CAS 48918 (3), CAS 48919 (2), CAS 48920 (13), CAS 48921 (1), CAS 48932 
(64), CAS 48933 (43: 40-64). Malaysia: BPBM 21891 (2: 40, 41), BPBM 21980 (8: 32-47). Viet Nam: CAS UNCAT. (1: 47). Taiwan: BPBM UNCAT. (2: 49, 51), BPBM $23077(2: 49,64)$. Philippine Islands: CAS UNCAT. (3: 26-49), CAS 45893 (2: 53, 54), CAS 46041 (2), CAS-SU 32274 (2), LACM 37414-7 ( $1: 48$ ), LACM 37423-4 (1: 43), LACM 42456-14 (3), LACM 42461-12 (3), LACM 42485-11 (7), LACM 42490-22 (3), LACM 42491-21 (2), USNM 122456 ( 1 ), USNM 137860（1）, USNM 137861 (1), USNM 225120（7), USNM 225121（2）, USNM 225125（5), USNM 225132 (3), USNM 225133（3), USNM 225142 (2), USNM 228046（23), USNM 228047 (5), USNM 228048（26), USNM 228052（5), USNM 228052（5), USNM 229053 (86), USNM 228063 (2), USNM 228064 (11), USNM 228065 (1), USNM 228066（8: 54-63), USNM 228101 (16), US NM 228110（4), USNM 228111 (1), USNM 228112（1: 56), USNM $228295(1: 58)$, US NM $228296(2: 39,51)$, USNM $228276(1: 28)$. Indonesia: RMNH 17950 (2), RMNH 20283 (1: 23), RMNH 20783 (1: 11), USNM $228093(10: 28-42)$, USNM 228095 ( 1 ), US NM $228096(6: 16-38)$, USNM 228103 ( 1 ), USNM 228105 (1), USNM 228273 (17: 17-45), USNM 228275 (2: 23, 4j). Australia, Western Australia: WAM P.20292 (2), WAM P.21291 (1), WAM P.22568 (1), WAM P.22626-33 (8), WAM P.22766-8 (3), WAM P.23956-9 (4), WAM P.24012-18 (7), WAM P.24275-9 (8), WAM P.24374-5 (2), WAM P.24606 (3), WAM P.25111-013 (14), WAM P.25112-017 (11), WAM P.25368-030 (1), WAM P.25813-025 (4), WAM P.25824-008 (1), WAM P.25827-002 (2), WAM P.26670-012 (7), WAM P.26677-016 (1). Australia, Northern Territory: AMS I.21961-020 (2), NTM UNCAT. (19: 20-62), NTM UNCAT. ( $15 ;$ HL 82-58), NTM UNCAT. (138; HL 82-71), NTM UNCAT. (3; HL 82-74). Australia, Queensland: AMS I.20770-101 (1: 36), USNM 164966 (8: 36-69), USNM $164967(26: 30-56)$, USNM 174345 (26: 37-75). 
Papua-New Guinea: USNM UNCAT. (4: 17-21), USNM 212137 (1), USNM 212140 (1), USNM 227629 (1), USNM 228097 (2), USNM 228098 (3: 28-29), USNM 228102 (2), USNM 228107 (2: 30, 32), USNM 228108 (30 of 66 examined: 32-51), ZMB 15141 (1). Bismarck Archipelago: CAS 48892 (2), USNM 228099 (22: 35-56), USMM 228106 (3). Solomon Islands: AMS I.17486-005 (3: 39-47), ВРвM 16022 (6: 17-43), USNM 122988 (2).

\section{Cirripectes fuscoguttatus strasburg and Schultz}

Cirripectus fuscoguttatus Sirasburg and Schultz, 1953:130 (Rongerik Atoll, Eniwetak Island).

Diagnosis. A species of Cirripectes with the following combination of characters: broadly expanded nuchal flap bearing distinct cirri (type C; Figure 7), 0-5 lateral line tubes, and males and females with dark-brown spots on a pale-brown background.

Description. Dorsal fin XII,13-15 ( 14 in $97.1 \%$ of specimens); anal fin II, 14-16 (15 in 96.4\%); total procurrent caudal-fin rays 11-14; pelvic fin $I, 4$; vertebrae $10+20=30$; last pleural ribs on vertebral centrum 11-12 (11 in 99.3\%); last epipleural rib on vertebral centrum 17-20 (17-19 in 97.8\%); anal pterygiophores $1-1-2$ or $1-2-1$ (1-1-2 in $82.3 \%)$; nuchal cirri $47-66$ (50-60 in $93.3 \%$ ); supraorbital cirri $18-42$ (20-37 in 89.6\%); nasal cirri $7-32$ ( $11-27$ in $86.4 \%$; LL tubes $0-5$ ( $0-3$ in $95.8 \%)$; last LL tube positioned at point between verticals from dorsal-fin ray 13 and caudal-fin base (at caudal-fin base in $75.6 \%$ ); no 
scalelike flaps along LL; lower lip swuoth mesially (plicate laterally); gill rakers 22-32 (based on 46 specimens); pseudobranchial filaments on one side 8-13 (based on 46 specimens); premaxillary teeth 248-310 (based on 11 specimens); dentary teeth 120-140 (based on 11 specimens); upper lip crenulae approximately 40-55; nuchal cirri in four groups, two or more groups occasionally connected basally by a slightly notched frenum, dorsalmost groups of cirri usually meet or overlap at midpoint on nape, ventralmost group of cirri on each side borne on expanded flap (type C; Figure 7); first dorsal-fin spine of adults equal to or sightly longer than second $(0-4.4 \mathrm{~mm}$ longer in males, 0-2.9 m longer in females); dorsal-fin membrane deeply incised above last dorsal-fin spine; dorsal-fin membrane attached to caudal fin in adults; cephalic pore system complex ( 3 or more pores at most positions; number of pores increases with increasing SL); mid-snout pores present (Figure 17); extra interorbital pore position present (Figure 17); two pore positions behind nuchal flap (Figure 17); male genital papilla with urogenital orifice located basally between two widely separated slender filaments (less than 1.0 min long) on a fleshy swelling behind anus (type I; Figure 13); testes bulbous, length equals width; maximum SL about $106 \mathrm{~mm}$; ophioblennius larvae not known.

The smallest mature female (ova about 0.6 m diameter) examined was about 56 mn SL. Males mature by about 50 mm SL.

Color in alcohol. Small specimens (10-30 um SL) have body and head markings similar to young Cirripectes gilberti (see next species account), except young $\underline{C}$. Euscoguttatus have the broad white triangle beneath the eye extending dorsally along posterior margin of orbit to 
top of head (Figure 35). One 23 m SL specimen from the Marshall Islands has faint dark-brown spots on body instead of typical uniformly brown pattern of juveniles.

As size of males and females increases, number of dark-brown spots on head and body increases; background color of body behind spots becomes blotched, forming about four broad irregular brown bars with pale interspaces (Figure 36). More details of changes in color pattern with size are discussed by Strasburg and Schultz (1953).

Color in life. Adult males (based on a color slide of a Marshall Islands speciumen) with dark-brown spots over head and body; head and anterior half of body with yellowish background, becoming brown on posterior half of body; anterior dorsal-fin spines yellowish, fin otherwise with indistinct brown spots and bars, and pale stripe extending entire length of basal fourth of fin; segmented-dorsal fin otherwise with dark-brown rays and dusky membranes; tips of upper caudal-fin rays orange, remainder of fin dark brown; anal fin dark brown; anal-fin spine rugosities yellowish brown; pelvic fin yellowish; pectoral fin with dark-brown spots on proximal half, distal half of upper rays brown with pale interradial membranes, distal half of lower rays yellowish brown; nuchal cirri black; supraorbital cirri yellowish brown; nasal cirri brown; iris orangish red.

Distribution. Cirripectes fuscoguttatus is widely distributed on the Pacific plate and occurs along the plate margin at Taiwan and Niuatoputapu Island, Tonga Islands. It is not known to occur at Johnston Island, the. Hawaiian Islands, Line Islands, or several other groups (Figure 22). Typically, it is found on coral reefs in the surge zone at depths of $0-8 \mathrm{~m}$. 
Comparisons. Cir:ipectes fuscoguttatus differs from all Cirripectes species occurring on the Pacific plate in having a broadly expanded nuchal flap bearing distinct cirri (type C; Figure 7). In Taiwan, where the geographic ranges of $\underline{C}$. fuscoguttatus and $\underline{C}$. imitator overlap, both have a large nuchal flap, but the latter usually has 7-14 lateral line tubes (versus 0-5). Cirripectes fuscoguttatus differs from its Indian Ocean sister species, $\underline{C}$. gilberti, only in color pattern, as discussed in the account of the latter.

Etymology. The specific epithet is a combination of the Latin fuscus, meaning dusky or dark, and guttatus, meaning spotted, in reference to the dark brown spots on head and body.

Material examined. Types: USNM 113634 ( 1 female: 68 mm SL), holotype of Cirripectes fuscoguttatus, Marshall Islands, Rongerik Atoll, Eniwetak Islet, ocean reef; and the following paratypes of Cirripectes fuscoguttatus: USNM 115497 (1: 75), Samoa Islands, Tutuila Island; USMM 115498 (5: 75-90), Phoenix Islands, Enderbury Island reef; USNM 142102 (10: 19-94), Marshall Islands, Bikini Atoll, Eman Islet; USNM 142103 (16: 23-86), Marshall Islands, Bikini Atol1, Airy Islet; USNM 142104 (35: 23-97), USNM 142105 (1: 72), Marshall Islands, Kwajalein Atoll, Ennylabegan Islet; USNM 142106 (11: 22-82), Marshall Islands, Kwajalein Atol1, Ennylabegan Islet; USNM 164960 (2: 60-79), Gilibert Islands, Onotoa Atoll.

Other material examined. Pacific Ocean (exact locality unknown): USNM 198700 (3: 63-79). Taiwan: USNM 227869 (1: 106). Caroline Islands: CAS 489422 (4: 39-82), CAS 48937 (13), CAS 48940 (7), CAS 48943 (15: 41-106). Mariana Islands: BPBM UNCAT. (2: 69-81), CAS 
$48915(1: 27)$, CAS 48931 (5: 77-96). Marsha11 Islands: BPBM 8008 (10), BPBM 15517 (1), LACM UNCAT. (1; MARSHALL IS.), LACM 4067 (4: 50-79), LACM 6679-37 (3), USNM 200612 (3: 29-67), USNM 227864 (1: 88). Phoenix Islands: USNM 201888 ( $1: 82$ ), USNM 227865 (2: 26-28), USNM 227870 (1: 34). Tonga Islands: USNM 227868 (3: 54-86). Samoa Islands: LACM 34356-11 (3: 85-94). Tuamoto Archipelago: BPBM UNCAT. (4: 59-77), CAS 48928 (2), CAS 48929 (2), CAS 48954 (1), USNM 227866 (1: 93), USNM 227867 (4: 92-97).

\section{Cirripectes gilberti new species}

Diagnosis. A species of Cirripectes with the following combination of characters: type C nuchal cirri pattern (Figure 7), high number of nuchal cirri (typically more than 45), 7-14 lateral-1ine tubes, adult males with uniformly brown body, and females with reddish spots in life (pale in alcohol).

Description. Dorsal fin XII, 14; anal fin II, 15; total procurrent caudal-fin rays 12-14; pelvic fin I,4; vertebrae 10+20=30; 1ast pleural ribs on vertebral centrum 11; last epipleural rib on vertebral centrum 17-19; anal pterygiophores $1-1-2$ or $1-2-1$ (1-1-2 in $95.8 \%$ of specimens); nuchal cirri 50-64 (53-59 in $82.6 \%$ ); supraorbital cirri 17-48 (22-35 in $79.2 \%)$; nasal cirri 11-33 (11-23 in 95.8\%); LL tubes 0-5 (0-3 in 95.8\%); last LL tube positioned at point between verticals from dorsal-fin ray $\bar{y}$ and caudal-fin base (on caudal-fin base in 76. 2\%); no scalelike flaps along LL; lower lip smooth mesially (plicate lateral1y); gil1 rakers $24-30$ (based on 23 specimens); pseudobranchial 
filamests on one side 8-13 (based on 23 specimens); premaxillary teeth 203-270 (based on 16 specimens; number of teeth increases with increasing SL; specimens greater than 60 m SL have 240-270 teeth); dentary teeth 100-141 (based on 17 specimens; specimens greater than 60 mm SL have more than 120 teeth); upper lip crenulae approximately 36-48; nuchal cirri in four groups, two or three groups occasionally connected basally by a slightly notched frenum, dorsalmost groups of cirri usually overlap at midpoint on nape, ventralmost group of cirri on each side borne on expanded nuchal flaps (type C; Figure 7); first dorsal-fin spine of adults slightly longer than second $(0.1-2.6 \mathrm{~mm}$ longer in males, $0.4-4.3$ mon longer in females); dorsal-fin membrane deeply incised above last dorsal-fin spine; dorsal-fin membrane attached to caudal fin in adults; cephalic pore system complex ( 3 or more pores at most positions; Figure 17; number of pores increases with increasing SL); mid-snout pores present (Figure 17); extra interorbital pore position present (Figure 17); two pore positions behind nuchal flap (Figure 17); male genital papilla with urogenital orifice locacté basally between two widely separated slender filaments (less than 1.0 mm long) on a fleshy swelling behind anus (type I; Figure 13); testes bulbous, length equals width; maximum SL about $102 \mathrm{~mm}$; ophioblennius larvae not known.

The smallest mature female (ova about 0.6 mm diameter) examined was about 76 min SL (a 50 SL specimen was still immature). Males mature by about 50-60 ant SL. 
Color in alcohol. Color pattern changes ontogenetically and differs sexually among adults (Figures 37 and 38 ). Body of specimens less than $40 \mathrm{~mm}$ SL (Figure 39) uniformly brown, with faint dark-brown spots beginning to appear posteriorly on specimens nearing $40 \mathrm{~mm}$ SL; head with brown background, broad white triangular area extending from point at mid-ventral margin of orbit ventrally over posteriormost half (as measured from snout tip) of upper lip onto lower lip, narrow white bar reaching from anterior edge of orbit over lip at snout tip where it almost meets similar bar from other side forming a $V$ on snout; dorsal fin dusky with pale triangular area at tips of anterior spines; anal fin dark brown; caudal fin dusky; pectoral-fin rays dusky; pelvic-fin rays dusky; nuchal cirri black; supraorbital and nasal cirri dark brown.

Between 40-65 m SL, females have pupil-sized dark-brown spots over body and slightly smaller ones on head; males have darker brown background color and sometimes very faint spots or irregular pale-brown bars on body; pale triangular wedge beneath eye present but daiker in both sexes; females with small brown spots scattered over wedge; pale bars at snout tip faint or absent.

Females longer than 65 m. SL with head covered with 1-2 man diameter spots on dark-brown background, spots extending onto pectoral fin and ventral halves of dorsal and anal fins; spots on fins, head, and anterior half of body pale, becoming dark brown on posterior half of body; uppermost caudal-fin rays pale, remainder dark brown.

Males greater than 65 min SL with uniformly dark-brown head and body; tips of dorsal-fin elements pale, remainder dark brown; anal fin 
dark brown; tips of uppermost caudal-fin rays pale, remainder of fins dark brown; anal-fin rugosities dark brown.

Color in life. Adult females (based on a color slide of a Chagos Archipelago specimen) have background color of head and body mottled with pale-olive and dark-brown blotches; stomach and throat white; head and anterior half of body with reddish-orange spots becoming reddish brown at mid-body and dark brown near caudal-fin base; orangish-brown spots on dorsal, anal, proximal half of caudal, and pectoral fins; ventralmost pectoral-fin rays with orange tips; dorsal fin with tips of spines yellow and tips of rays orange; tips of dorsalmost caudal-fin rays bright orange, remainder of fin dusky orange; iris red except for yellow ring around pupil.

Life colors of males unknown.

Comparisons. Cirripectes gilberti is closely related to $\underline{C}$.

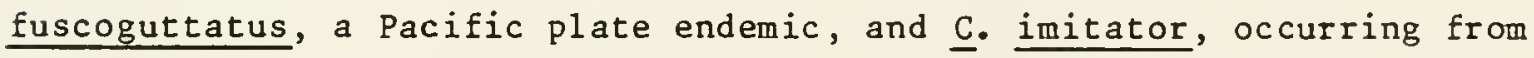
Taiwan to Japan. All three have a type $C$ nuchal cirri pattern (Figure 7), and high number of nuchal cirri (typically more tinan 45). Cirripectes imitator differs from the other two in having 7-14 lateral-line tubes (versus 0-5). Cirripectes gilberti and $\underline{\mathrm{C}}$. fuscoguttatus appear to differ only in color pattern. Adult males of C. gilberti do not have spots, and females have reddish spots in life (pale in alcohol), whereas both sexes of $\mathrm{C}$. fuscoguttatus have dark-brown spots (in life and alcohol).

of those Cirripectes species occurring in the Indian Ocean, $\underline{C}$. gilberti is the only one that has a type $C$ nuchal cirri pattern. 
Distribution. I have examined specimens of Cirripectes gilberti from seven Indian Ocean localities: South Africa, Comoros Islands, Agalega Island, Seychelles Islands, Charos Archipelago, Cocos (Keeling) Islands, and the northern tip of Sumatra. The male identified by Smith

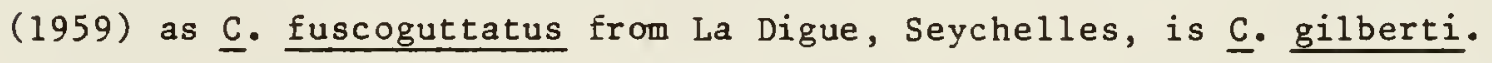
Cirripectes gilberti occurs on rocky and coralline substrates at depths ranging from near the surface to $8 \mathrm{~m}$.

Etymology. This species is named for Dr. Carter R. Gilbert (FSM), who provided assistance and encouragement during the course of this study.

Comments. Although Cirripectes gilberti is similar to $\underline{\mathrm{C}}$. fuscoguttatus, their strikingly different color patterns and non-overlapping geographic distributions warrant their recognition as separate species.

Material examined. Holotype: USNM 274749 (male: 82 m SL), Indonesia, Pulo Boenta, off Achen Head, Sumatra, $05^{\circ} 33^{\prime} 10^{\prime \prime} \mathrm{N}$, $095^{\circ} 09^{\prime} 30^{\prime \prime} \mathrm{E}$, surge channel in coral reef, $0.4 \mathrm{~m}, 20 \mathrm{Nov} .1963$, "Te Vega" Cruise 2, station 93.

Paratypes: USNM 227872 (2: 71,90), collected with the holotype; ANSP 134747 (5: 34-45), Cocos-Keeling Islands, North Keeling Island, $11^{\circ} 50^{\prime} \mathrm{S}, 096^{\circ} 49^{\prime} 30^{\prime \prime} \mathrm{E}, 0-1 \mathrm{~m}, 6$ Mar. 1974; ROM 46597 (6: 76-93), Chagos Archipelago, Salomons Group, Isle Boddam, orean side at southwest tip of island, $05^{\circ} 21^{\prime} 05^{\prime \prime} \mathrm{S}, 072^{\circ} 12^{\prime} 12^{\prime \prime} \mathrm{E}, 0-3 \mathrm{~m}, 18$ Mar. 1979; RUSI 15299 ( $1:$ 102), Seychelles Islands, Farquhar Group, La Digue, 12 Oct. 1954; USNM 227871 (3: 43-47), Agalega Island, North Island, about one-half mile southwest of tip, dead coral channels, some 
live coral, $10^{\circ} 19^{\prime} \mathrm{S}, 056^{\circ} 35^{\prime} \mathrm{E}, 0-9 \mathrm{~m}, 17$ April 1976; CAS 40379

(5: 34-102), Comoros Islands, Grande Comore Island, N'Gouni Reef, about one-half km north of Iconi, surge channel at far end of small cove, 0-5 m, 1 Mar. 1975; RUSI UNCAT. (1: 49), South Africa, Boteler Point, 13 April 1979.

\section{Cirripectes hutchinsi new species}

Diagnosis. A species of Cirripectes with the following combination of characters: complex cephalic sensory pore pattern, I, 4 pelvic-fin rays, no extra interorbital pore position (Figure 17), 36-42 nuchal cirri.

Description. Dorsal fin XII-XIII (XII in $94.7 \%$ of specimens), 13-14 (14 in 89.5\%); anal fin II, 15-16 (15 in 89.5\%); total procurrent caudal-fin rays 9-11; pelvic fin 1,4 ; vertebrae $10+20-21$ (20 in $94.7 \%)=30-31(30$ in $94.7 \%)$; last pleural ribs on vertebral centrum 11; last epipleural rib on vertebral centrum $16-20$ (17-19 in $89.5 \%$ ); anal pterygiophores $1-1-2$ or $1-2-1$ ( $1-1-2$ in $89.5 \%$ ) nuchal cirri $35-42$ (36-41 in $89.5 \%)$; supraorbital cirri $12-29$ (17-29 in $94.7 \%)$; nasal cirri 8-13 (12-26 in 89.5\%); LL tubes $3-9$; last LL tube positioned at point between verticals from dorsal-fin ray 13 and caudal-fin base (on caudal-fin base in $82.4 \%$ ); no scalelike flaps along LL; lower lip smooth mesially (plicate laterally); gill rakers 23-29 (based on 18 specimens); pseudobranchial filaments on one side 8-14 (based on 19 specimens); premaxillary teeth 192-218 (based on 10 specimens); dentary teeth 97-114 (based on 10 specimens); upper lip crenulae approximately 
36-42; nuchal cirri in four groups with each ventralmost group of cirri borne on a slightly expanded nuchal flap (type B; Figure 7); first dorsal-fin spine of adu?ts slightly longer than second $(1.5-11.0 \mathrm{~mm}$ longer in males, 0.7-4.9 mm longer in females); dorsal-fin membrane deeply incised above last dorsal-fin spine; dorsal-fin membrane attached to caudal fin in adults; cephalic pore system complex (more than 3 pores at most positions; Figure 17; number of pores increases with increasing SL); mid-snout pores present (Figure 17); extra interorbital pore position absent (Figure 17); 2 pore positions behind nuchal flap (Figure 17); male genital papilla with urogenital orifice located basally between two widely separated slender filaments (less than 1.0 long) on fleshy swelling behind anus (type I; Figure 13); testes bulbous, length equals width; maximum SL about 105 mm; ophioblennius 1 arvae not known.

The smallest mature female (ova about $0.5 \mathrm{~mm}$ diameter) examined was about $73 \mathrm{~mm}$ SL. Males mature by about 60-70 m SL.

Color in alcoho1. Young specimens (30-40 man SL) have pale brown areas on dorsal and ventral thirds of body with mid-lateral third darker brown, section of body between pectoral-fin base and posterior end of anal-fin base overlaid with dark-brown spots and narrow wavy lines; pale-brown area on body at caudal-fin base; head brown with small pale spots on snout and lower part of cheeks, 6-8 narrow pale bars on upper 1ip; throat with brown bar across middle, flanked by pale areas anteriorly and posteriorly; transluscent triangular area at tips of anterior dorsal-fin spines and over upper and lower caudal rays; tips of posterior dorsal-fin elements pale, remainder of fin dusky; 
middle of caudal fin dusky; lower half of pectoral fin dusky, upper half transluscent; proximal half of pelvic fins dusky, distal half transluscent; nuchal cirri dark brown; nasal and supraorbital cirri brown.

Adult females have a body color pattern that varies geographically from north to south (Figures 40 and 41 ). The southern pattern consists of small pupil-sized spots arranged over broad mid-lateral section of body from behind head to posterior end of anal-fin base, and pale area extending from posterior end of anal-fin base to caudal-fin base. In the northern pattern, about five to nine slightly-irrejular broad dark-brown bars with pale interspaces extend from dorsum to about two-thirds distance to anal fin base; anterior half of body has small dark-brown spots overlying barred pattern. Amount of body covered by spots variabe, with some specimens showing patterns intermediate between the two patterns. All females with two to five vertical rows of small dark-brown spots on proximal half of pectoral fin; head brown with two or three diagsnal dark-brown bars extending from nape anteroventrally to middle of head where bars break up into small spots that cover throat and ventral half of head; upper 1 ip with 8-10 pale bars; middle of spinous dorsal fin with line of brown spots on interradial membranes; remainder of pattern same as for young specimens.

Adult males nave two color patterns (Figure 42), both unlike females; both patterns may be found in the same geographic area. One pattern consists of about six broad dark-brown bars with brown interspaces. In the other pattern, the body is uniformly dark brown 
without spots on the body. These patterns appear to be related to size, as the largest males have uniformly brown bodies, and most of the smaller nales have the barred pattern. All males have a brown head without dark spots; upper 1ip with 8-10 pale bars; no spots on pectoral fins; other colors similar to those of females and young specimens. Color in life. Females (based on color slides of specimens from Rottnest Island, Western Australia) with background color of head and body olive to brown; reddish-brown to dark-brown spots on head and body; spinous-dorsal fin dusky to dark brown, remainder varying from completely orange or red to dark brown with tips of rays red; caudal fin with dorsalmost and ventralmost rays red, middle rays brown; posterior part of body at caudal-fin base reddish; anal fin dark brown with brilliant blue tips; iris varies from yellow to orange.

Based on a color slide of a $57 \mathrm{~mm}$ SL specimen from Rottnest Island, a male with the barred color pattern resembles color in alcohol except: throat and edge of operculum yellowish; cheek brown; two anteroventraliy directed bars (becoming spots beneath eye) extend from top of head onto upper lip, interspaces yellow to brown; pectoral-fin base reddish brown; middle of spinous-dorsal fin with narrow brownish-orange line, spines yellow with tips of posterior spines becoming scarlet; segmented dorsal fin with diagonal posterodorsally-directed bromish-orange line on basal half of each interradial membrane, rays dusky yellow; anal fin with alternating lengthwise stripes in sequence from base: blue, reddish brown, blue, reddish brown; anal ray tips pale blue; pelvic fin reddish brown; ventralmost 5-6 nuchal cirri yellowish orange, others dark brown; other 
cirri brown; iris varies from completely orange to haviag inner yellow ring and outer orangish-red ring with black area in between.

Based on a color slide of a Shark Bay, Western Australia, specimen, a larger male resembles Rottnest Island male except for dorsal fin, which has anterior 4-5 spines yellow and remainder of fin scarlet; body is uniformly dark-brown.

Comparisons. Cirripectes filamentosus is the only Cirripectes species known to occur within the geographic range of $\underline{c}$. hutchinsi.

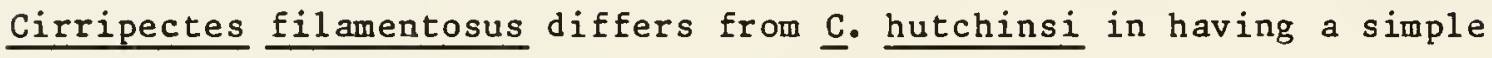
cephalic sensory pore pattern and males with a type II (Figure 13) genital papilla (versus type I).

Cirripectes randalli and females of $\underline{\text { C. hutchinsi, }} \underline{\text { C. perustus, and }}$ C. gilberti all occur in the Indian Ocean and have spots on the body.

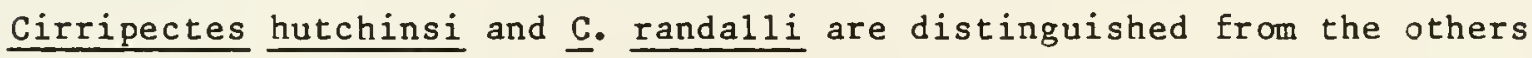
in the account of $\underline{c}$ randalli. Cirripectes hutchinsi and $\underline{\mathrm{C}}$ randalli differ from each other primarily in color pattern as discussed in the account of $\underline{\text { C. }}$ randalli.

Distribution. Cirripectes hutchinsi is known only from Western Australia, where it occurs from North West Cape southward to Rottnest Island, near Perth (Figure 22). It has been collected at depths ranging from one to $22 \mathrm{~m}$. Barry Hutchins has informed me (in litt.) that he has found this species in the surge zone in limestone holes just below the intertidal range, and commonly in areas where Pocillopora damicornis is present on the reefs.

Etymology. This species is named for Mr. Barry Hutchins of the Western Australian Museum, who collected most of the known specimens, 
provided information on the ecology of this species, and gave me color slides of both sexes.

Material examined. Holotype: WAM P.25744-003 (male: $89 \mathrm{~mm}$ SL), Australia, Western Australia, Rottnest. Island, Strickland Bay, 1 Mar. 1977.

Paratypes: AMS I.20239-023 (2: 85-104), Australia, Western Australia, Rottnest Island, Salmon Bay, $32^{\circ} 01^{\prime} \mathrm{S}, 115^{\circ} 27^{\prime} \mathrm{E}, 1-10$ m, 10 April 1978; AMS I.20243-004 (1: 82), Australia, Western Australia, Rottnest Island, $32^{\circ} 03^{\prime} \mathrm{S}, 115^{\circ} 26^{\prime} \mathrm{E}, 20-22 \mathrm{~m}, 11$ April 1978; USNM 273891 (2: 69, 79), Australia, Western Australia, Northwest Cape, 22 May 1980; WAM UNCAT. (1: 53; OUT OF 25744-003), collected with holotype; WAM P.4647 (1: 73), Australia, Western Australia, Point Quobba, Oct. 1959; WAM P.4652 (1: 98), Australia, Western Australia, Houtman Abrolhos, Wallaby Group, Pigeon Island, May 1959; WAM P.25317-002 (2: 90-106), Australia, Western Australia, Wallabi Group, Abrolhos Islands, Long Island, 21 May 1975; WAM P.25374-042 (1: 69), Australia, Western Australia, Northwest Cape, lagoon off Tantabiddi Creek, 3-4 m, 2 July 1975; WAM P.25758-010 (1: 73), Australia, Western Australia, Rottnest Island, Fish-Hook Bay, 8 Mar. 1977; WAM P.26072-003 (2: 32-52), Australia, Western Australia, Houtman Abrolhos, Beacon Island, outer reef near Seal Island, $28^{\circ} 29^{\prime}$ S, $113^{\circ} 47^{\prime} \mathrm{E}, 4 \mathrm{~m}, 10$ April 1978; WAM P.26626-001 (1: 76), same data as USNM 273891; WAM P.26657-009 (3: 34-70), Australia, Western Australia, Shark Bay, Steep Point, at entrance to South Passage, $26^{\circ}$ $08^{\prime} \mathrm{S}, 113^{\circ} 08^{\prime} \mathrm{E}, 13-15 \mathrm{~m}, 9$ April 1979. 


\section{Cirripectes imitatcr Williams}

Cirripectes imitator Williams, 1985:533 (Ogasawara Islands, Chichi Jima Island).

Diagnosis. A species of Cirripectes with the following combination of characters: 45 or more nuchal cirri, enlarged nuchal flap on either side of the head, 7-14 lateral line tubes, and color pattern consisting of pale spots on a dark background.

Description. Dorsai fin XI-XIII (XII in $95.2 \%$ of specimens), 13-15 (14 in 95.2\%); total dorsal-fin elements 26; anal fin II,14-16 (15 in $95.2 \%)$; total procurrent caudal-fin rays $11-14$; pelvic fin $1,3-4$ (bilaterally I, 4 in $88 \%$, bilaterally I, 3 in $6 \%$, one side I, 3 and other I,4 in 6\%); vertebrae $10+20=30 ;$ last pleural ribs on vertebral centrum 11; last epipleural rib on vertebral centrum 18-21 (19-20 in $85.5 \%$ ); anal pterygiophores $1-1-1,1-1-2,1-2-1$ (1-1-2 in 92.1\%); nuchal cirri 40-59 (45-53 in $82.4 \%)$; supraorbital cirri $13-46$ (15-35 in $82.7 \%$ ); nasal cirri $9-93(9-33$ in $84.6 \%)$; LL tubes $5-14(8-13$ in $94.6 \%)$; last LL tube positioned at point between verticals from dorsal-fin ray 9 and caudal-fin base (on caudal-fin base in $84.5 \%$ ); no scalelike flaps along LL; lower lip smooth mesially (plicate laterally); gil1 rakers $24-27$ (based on 22 specimens); pseudobranchial filaments on one side 7-9 (based on 22 specimens); premaxillary teeth 192-230 (based on 11 specimens); dentary teeth 84-120 (based on 11 specimens); upper 1ip 
crenulae approximately $34-50$; nuchal cirri in four groups (two on each side) separated at dorsalmost point on nape and about halfway down length of row on each side (gaps less than about $0.5 \mathrm{~mm}$ ), two groups on one side rarely connected by a low basal membrane, ventralmost group of cirri on each side borne on a broad nuchal flap; adults of both sexes with first dorsal-fin spine slightly longer than second ( $1-5$ m 1 longer in males, 1-2 monger in females); dorsal-fin membrane deeply incised above last dorsal-fin spine; dorsal-fin membrane attached to caudal fin in adults; cephaiic pore system complex (numerous pores at most positions; number of pores increases with increasing SL); mid-snout pores present (Figure 17); extra interorbital pore position present (Figure 17); pore positions behind nuchal cirri flap 2 (Figure 17); male genital papilla with urogenital orifice located basally between two widely separated slender filaments (less than 1.0 mim long) on a fleshy swelling behind anus (Figure 13); testes bulbous, length equals width; maximum SL about $100 \mathrm{~mm}$.

The smallest mature female (ova about $0.5 \mathrm{~mm}$ diameter) examined was 55 Im SL, from the Ogasawara Islands. Some 40-50 mm SL females have large ovaries, but they are granular in appearance and have no large ova. Males mature by about 50 min SL.

Color in alcohol. Color pattern highly variable, ranging from alternating dark- and pale-brown bars to dark-brown reticulations around pale-brown pupil-sized spots on body. Adult males (Figure 43) tend to be darker overall, with spots calescing into pale bars on body. Females (Figure 44) generally with reticulated color pattern, but both sexes can exhibit either pattern. Both sexes with cheeks, 
snout, upper lip, and urderside of head with pale-brown spots on darker-brown background or the reverse; some specimens with spots on underside of head fusing into 2 or 3 alternating dark and pale bars across throat; dorsal fin with translucent triangular area in anterodorsal part of spinous section, narrow pale stripe running through middle of fin to last dorsal spine, remainder of spinous and soft dorsal fin brown; upper part of caudal fin with translucent triangular area, remainder of fin brown; anal fin brown, tips of rays paler; pectoral and pelvic fins dusky; nuchal cirri black, other cirri brown. kugosities on anal-fin spines of males pale brown.

Color in life. Male (based on fig. 392-7a in Shen 1984, which he referred to as Cirripectes sebae) with alternating brown and bluish-white bars on head and body; those on head broken into irregular pupil-sized spots; pale section of caudal and spinous-dorsal fins with orange rays; red stripe running through middle of spinous dorsal; each nuchal cirrus with pale-yellow band near base; iris with yellow ring around pupil; other colors same as in alcohol. Fukao (1984) states that the Ogasawara specimens have bright-yellow spots or blotches on body in life.

Ferales (based on fig. $392-7 b$ in Shen 1984; and plate 87-D in Masuda et al. 1975) similar to male except spots on body not fused into bars, spots becoming smaller posteriorly. There is some doubt about the identification of the specimen in figure 392-7b (Shen 1984). I tentatively identify it as Cirripectes imitator, but it could be a specimen of $\underline{\mathrm{C}}$. castaneus, a species in which females have a very similar color pattern. 
Distribution. Cirripectes imitator is known to occur from Taiwan northward to Shirahama, Japan, and in the Ogasawara Islands (Figure 22).

Comparisons. Cirripectes imitator belongs to the $\underline{\text { C. }}$ fuscoguttatus complex of species, with which it shares two derived characters: a high number of nuchal cirri (usually 45 or more) and an enlarged nuchal flap on either side of the head. A large nuchal flap is also present

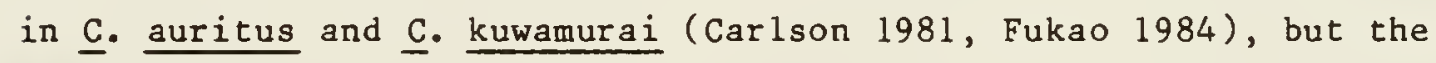
tuchal flaps in these species are proportionally larger, bearing poorly drveloped cirri, and are probably independently derived. Cirripectes

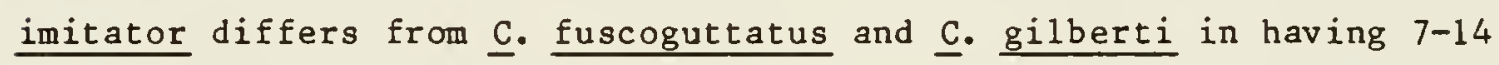
(in $98.2 \%$ of specimens; 1 of 56 with 5 ) LL tubes, versus $0-4$ (in $98.5 \%$; 2 of 69 with 5). The color pattern of $\underline{\text { C. }}$ imitator, with pale spots on a dark background, is the reverse of $\underline{\mathrm{C}}$. fuscoguttatus, which has dark spots on a pale background.

Williams (1985) discussed the differences between Cirripectes imitator and other sympatrically occurring congeneric species.

Etymology. The specific epithet is derived from the Latin imitor, meaning mimic, and refers to the similarity of the color pattern to that of Cirripectes polyzona and $\underline{C}$. castaneus.

Comments. No geographic variation was found for the meristic ur morphometric characters examined. Fukao (1984) noted that his Cirripectes polyzona specimens from the Ogasawara Islands $(=\underline{C}$. imitator at that locality) had bright-yellow spots or blotches in life. I attribute this color variant to populational variation within the species. 
Material examined. Holotype: FAKU 48203 (male: 65.6 um SL), Ogasawara Islands, Chichi Jima Island (approximately $27^{\circ} 30^{\prime} \mathrm{N}$, $\left.142^{\circ} 30^{\prime} \mathrm{E}\right)$, Sakiaura; $0.5-5 \mathrm{~m} ; 8$ April 1974. Other material examined is 1 isted in Williams (1985) and is not repeated here.

\section{Cirripectes jenningsi Schultz}

Cirripectes jenningsi Schultz, 1943:274 (Swains Island).

Diagnosis. A species of Cirripectes with the following combination of characters: uninterrupted row of nuchal cirri (type F; Figure 7), dorsal fin attached to caudal fin in adults, and more than 36 nuchal cirri.

Description. Dorsal fin XII-XIII (XII in $98.9 \%$ of specimens), 15-16 ( 15 in $93.6 \%)$; anal fin II, $15-17$ (16 in $95.7 \%$ ); total procurrent caudal-fin rays 10-14; pelvic fin 1,4 ; vertebrae $10+21-22$ (21 in $92.6 \%)=31-32(31$ in $92.6 \%)$; last pleural ribs on vertebral centrum 11-13 (12 in 97.9\%; one each with 11 and 13); last epipleural rib on vertebral centrum 20-23 (21-23 in 95.7\%); anal pterygiophores $1-1-1$, $1-1-2$, or $1-2-1$ (1-1-2 in $88.2 \%)$; nuchal cirri $34-44$ (37-42 in $93.0 \%)$; supraorbital cirri $2-4$ (2-3 in $96.5 \%)$; nasal cirri $8-19$ (9-12 in $94.3 \%)$; LL tubes $2-11$ (5-9 in $93.9 \%)$; last LL tube positioned at point between verticals from dorsal-fin ray 15 and caudal-fin base (on caudal-fin base in 95.7\%); no scalelike flaps along LL; lower lip crenulate mesially (plicate laterally); gill rakers 19-24 (based on 25 specimens); pseudobranchial filaments on one side 9-11 (based on 25 
sDecimens); premaxillary teeth 244-269 (based on 3 specimens); dentary teeth 112-119 (based on 3 specimens); upper lip crenulae approximately 41-47; nuchal cirri in one continuous row with cirri connected at their bases by a low membranous ridge, nuchal flap absent (type F; Figure 7); first dorsal-fin spine of adults equal in length or slightly longer than second (0-3.3 m longer in males, 0.9-2.7 m longer in females); dorsal-fin membrane deeply incised above last dorsal-fin spine; dorsal-fin membrane attached to caudal fin in adults; cephalic pore system complex ( 3 or more pores at most positions; Figure 17; number of pores increases with increasing SL); mid-snout pores present (Figure 17 ); extra interorbital pore position present (Figure 17); pore positions behind nuchal flap 2 (Figure 17); male genital papilla with urogenital orifice located basally behind a single slender filament (less than $1.0 \mathrm{~mm}$ long) on a fleshy swelling behind anus (type IV; Figure 13); testes bulbous, length equals width; maximum SL about 76 mm; ophioblennius larvae with 2 canines posteriorly on each dentary. The smallest mature female (ova about 0.6 um diameter) examined was about 42 SL. Males mature by about 45-50 SL.

Color in alcohol. Ophioblennius stage larvae (based on a $28.2 \mathrm{~mm}$ SL Raroia specimen) with cream colored head, body, cirri, and fins; small dark-brown spot (about half pupil diameter) iocated posteriorly on lower lip.

Juveniles and adults of both sexes have same color pattern (Figure 45): haad and anterior half of body pale with dark-brown pupil-sized spots, frequently with two broad brown bars on body; posterior half of body brown with pale pupil-sized spots; dorsal fin with transluscent 
area at tips of anterior dorsal. fin spines, posterior spines with dark-brown tips, basal third of segmented dorsal fin dark brown with white spots, pale stripe extending through middle, dusky above stripe; caudal fin brown with tips of dorsalmost rays pale; anal fin brown with pale spots on basal half; dorsal half of pectoral fin pale, ventral half brown; all cirri pale. Males have pale anal-fin spine rugosities. Color in life. I have not seen life colors of this species, but Schultz (1943) describes them for a specimen from Swains Island.

Comparisons. Cirripectes jenningsi is the only species in the genus with an uninterrupted row of nuchal cirri (type F; Figure 7). All other Cirripectes species have two or more groups of nuchal cirri separated by small gaps. Cirripectes jenningsi is endemic to the Pacific plate, where it is most similar to $\underline{C}$. quagga. Cirripectes quagga differs from $\underline{C}$. jenningsi in having two groups of nuchal cirri separated at midpoint of nape (type E; Figure 7), dorsal fin not attached to caudal fin, and usually less than 34 (in $96.9 \%$ of specimens) nuchal cirri (versus more than 36 in $95.8 \%$ ).

Distribution. Cirripectes jenningsi is known to occur from the Gilbert Islands to the Tuamotu Archipelago (Figure 19) on shallow coral reefs. One female (USNM 228160) is recorded from Aldabra Atoll, having been found together with a large series of $\underline{C}$. quagga and $\underline{C}$. variolosus. This group of specimens was accessioned at the USNM along with material collected at localities on the Pacific plate. Cirripectes variolosus is otherwise known only from the pacific plate and $\underline{\text { C. }}$ quagga, although widespread geographically, is rarely collected in the Indian Ocean and, when collected, is found in small numbers 
relative to other species of the genus. As no other specimens of $\underline{C}$. jenningsi are known from the Indian Ocean, I believe the data associated with USNM 228160 are erroneous and do not consider Aldabra as part of the geographic range of either $\underline{\mathrm{C}}$. jenningsi or $\underline{\mathrm{C}}$. variolosus. I know of one additional non-Pacific plate locality (New Caledonia) where C. jenningsi (MNHN 1980.224) has supposedly been collected, but these locality data are also questionable. A. Mauge (pers. comm.) received the material from $P$. Fourmanoir, in New Caledonia, and it was thought to be from that locality. However, as Fourmanoir has also made collections in the Tuamotu Archipelago, he could have collected the specimen there. Pending confirmation of the presence of $\underline{C}$. jenningsi in New Caledonia by new collections, I consider the distribution of this species to be restricted to the Pacific plate.

Etymology. Schultz (1943) named this species for a Mr. Jennings of Swains Island.

Comments. Schultz (1943) gave an anal-fin ray count of 18 for Cirripectes jenningsi. This count includes both spines and rays, whereas I separated the two types of rays in my formula. Schultz's count of 28 nuchal cirri on the holotype is lower than my lowest recorded value. The nuchal cirri on the holotype had been damaged prior to collection, therefore, I excluded this count.

Material examined. Types: USNM 115486 (male holotype: 63; female allotype: 65 SL), Tokelau Islands, Swains Island, 3-9 May 1939; USNM 115487 (20: 35-66), paratypes, same data as holotype. 
Other materiai examined. Pacific Ocean (exact locality unknown): USNM 198714 (1: 61). Gilbert Islands: USNM 167332 (1: 73). Phoenix Islands: USNM $216718(1: 49)$. Line Islands: USNM 200615 (45: 30-76). Tuamotu Archipelago: BPBM 14006 (4: 32-69), CAS 48916 (53: 28-67), CAS 48935 (3), CAS 48941 (27), CAS 48953 (17), USNM 228159 (2: $53,69)$.

The following two lots seem to have erroneous locality data: USNM 228160 ( $1: 40$ ), cataloged as being from Aldabra Atoll, but is probably from the Pacific Ocean (see earlier discussion); and MNHN 1980.224 (2), cataloged as being from New Caledonia, but is probably from the Tuamotu Archipelago according to A. Mauge.

\section{Cirripectes kuwamurai Fukao}

Cirripectes kuwamurai Fukao, 1984:106 (Shirahama, Japan). Cirripectes sp. Masuda, Araga, and Yoshino, 1975, 1980:267 (Shirahama, Japan).

Diagnosis. A species of Cirripectes with the following combination of characters: greatly expanded nuchal flap bearing ventralmost group of cirri on either side of nape, narrow pale stripes (red in life) on body.

Description. Dorsal fin XII,16; anal fin II, 17; total procurrent caudal-fin rays 10 ; pelvic fin 1,4 ; vertebrae $10+22=32$; last pleural ribs on vertebral centrum 1I; last epipleural rib on vertebral centrum 19; anal pterygiophores 1-1-2; nuchal cirri 30; supraorbital cirri 13; 
nasal cirri 10; LL tubes 5; last LL tube positioned at vertical from dorsal-fin ray 7; no scalelike flaps along LL; lower 1 ip smooth mesially (plicate laterally); gill rakers 23; pseudobranchial filamen:s on one side 8; premaxillary teeth 148; dentary teeth 76; upper 1ip crenulae approximately 41; ventralmost nuchal cirri borne on greatly enlarged nuchal flap, those cirri above flaps independent, no membranous ridge connects their bases (type A; Figure 7); first dorsal-fin spine about 0.5 morter than second; dorsal-fin membrane deeply incised above last dorsal-fin spine; dorsal-fin membrane attached to caudal peduncle in advance of caudal fin; cephalic sensory pore system simple ( $1-3$ pores at most positions; Figure 17); mid-snout pores present (Figure 17); extra interorbital pore position absent (Figure 17); pore positions behind nuchal flap 2 (Figure 17). Color in alcohol. Head and body (Figure 46) of holotype with brown background color; head with pale bar laterally on cheeks (very faint, slightly darker than background); body with five narrow stripes (slightly darker than background color) from above and behind pectoral-fin base to caudal-fin base; spinous dorsal fin dusky basally, pale distally; segmented-rayed dorsal fin with two faint dusky stripes in central part of fin; other fins dusky; enlarged nuchal flap black; all cirri brown.

Color in life. Head of holotype (based on color photo in Masuda et al., 1975, 1980) brown with scarlet reticulations; body brown with five scarlet stripes, dorsalmost stripe irregular and wavy; narrow red bar at base of pectoral-fin rays; dorsal fin with red spots in middle of fin on membranes between spines, two narrow red stripes extending 
Lhiough middle of anterior three-fourths of segmented dorsal fin, entire dorsal fin with narrow red distal margin; basal part of caudal-fin rays red, remainder of caudal-fin pale; anal fin ausky.

Comparisons. See Cirripectes auritus account for comparisons. Distribution. Cirripectes kuwamurai is known only from the holotype, which was collected on a rocky reef in water about 3 m deep off the northern beach of the Seto Marine Biological Laboratory, Shirahama, Wakayama Prefecture, Japan (Figure 20).

Etymology. This species is named for Dr. Tetsuo Kuwamura, collector of the only known specimen.

Comments. My counts for the holotype agree generally with those of Fukao (1984). The slight differences reflect counts that are difficult to make and should be considered approximate.

Material examined. Holotype: FAKU 48479 (female: $55 \mathrm{~mm}$ SL), Japan, Wakayama Prefecture, Shirahama, rocky reef on north shore of Seto Biological Laboratory, 3 m, 4 May 1974.

\section{Cirripectes obscurus (Borodin)}

Exallias obscurus Borodin, 1927:1 (Oahu).

Diagnosis. A species of Cirripectes with the following combination of characters: small scalelike flaps on the anterior part of the lateral line, pupil-sized dark spots behind each eye, 0-8 lateral line tubes, and last lateral line tube on caudal-fin base. 
Description. Dorsal fin XI-XII (XII in $98.0 \%$ of specimens), 15-17 ( 16 in $92.0 \%)$; anal fin II, 16-17 (17 in $95.5 \%)$; total procurrent caudal-fin rays $10-14$; pelvic fin $I, 4$; vertebrae $10+21-23$ (22 in $95.7 \%)=31-33(32$ in $95.7 \%)$; last pleural ribs on vertebral centrum 12; last epipleural rib on vertebral centrum 19-22 (19-21 in 97.9\%); anal pterygiophores $1-1-1,1-1-2$, or $1-2-1$ ( $1-1-2$ in $58.8 \%)$; nuchal cirri $36-47$ (38-44 in $86.4 \%)$; supraorbital cirri 10-26 (11-19 in $75.0 \%)$; nasal cirri $14-42(15-42$ in $96.0 \%)$; LL tubes $0-10$ (0-8 in $94.2 \%)$; last LL tube on caudal-fin base; irregularly spaced scalelike flaps along anterior part of $L L ;$ lower lip crenulate mesially (plicate lateral1y); gill rakers 26-30 (based on 25 specimens); pseudobranchial filaments on one side 11-15 (based on 25 specimens); premaxillary teeth 284-315 (based on 11 specimens); dentary teeth 145-158 (based on 11 specimens); upper lip crenulae approximately 41-50; nuchal cirri in two groups that sometimes overlap at midpoint on nape, no nuchal flaps (type E; Figure 7); first dorsal-fin spine of adults slightly longer than second (0.4-2.5 um longer in males, 0.4-4.5 mm longar in females); dorsal-fin membrane deeply incised above last dorsal-fin spine; dorsal-fin membrane attached to caudal peduncle in advance of caudal fin; cephalic pore system complex ( 6 or more pores at most positions; Figure 17; number of pores increases with increasing SL); mid-snout pores present (Figure 17); extra interorbital pore position present (Figure 17); pore positions behind nuchal flap 2 (Figure 17); male genital papilla with urogenital orifice located at distal tip of a slender filament (less than $1.0 \mathrm{~mm}$ long) on a fleshy swelling behind anus (type III; Figure 13); testes elongate, length equals two to three 
times width; maximum SL about $175 \mathrm{~mm}$ (based on Randal1, 1981);

ophioblennius larvae have not been examined.

The smallest mature female (ova about 0.4 m diameter) examined was about $110 \operatorname{man}$ SL, but females have we11-developed ovaries by about $85 \mathrm{~mm}$ SL. Males mature by about 75 min SL.

Color in alcohol. Adult males (Figure 47) with dark-brown background color; tiny dark or pale spots over head and anterior half of body, sometimes over entire body; pupil-sized black spot on each side of head posterior to eye; all fins dark brown; pectoral fin with elongate black area at base of upper 3-8 rays; all cirri dark brown to black.

Adult females and subadults (Figure 48) usually with mottled brown and pale pattern over head and body, body sometimes with about four to eight irregular bars, background pattern overlaid with small pale spots; other color characters as described for males.

\section{Color in life. Similar to color in alcohol except as follows} (based on color slides of a male and female fron yawaii): female head and body with tiny white spots becoming lavender posteriorly; underside of head golden brown; dorsal-fin spines red, dorsalmost and ventralmost caudal-fin rays dusky yellow, middle rays purplish red; anal fin purplish black with two narrow reddish stripes near middle of fin and running length of fin; tips of middle pectoral-fin rays dusky yellow, others brown; nuchal cirri bluish black; nasal and supraorbital cirri dark brown; iris yellowish. Male similar to female except spots not present on caudal-fin base; underside of head reddish brown; anal-fin stripes indistinct and brownish; tips of ventral pectoral-fin rays reddish brown; iris orange; anal-fin spine rugosities black. 
Comparisons. Cirripectes obscurus and $\underline{\mathrm{C}}$. alboapicalis are the only species of Cirripectes with small scalelike flaps on the anterior part of the lateral line and pupil-sized dark spots behind each eye. Characters differentiating these species are discussed in the account of $\underline{\text { C. }}$ alboapicalis.

Distribution. Cirripectes obscurus is known only from the Hawaiian Islands (Figure 19), where it is usually found on rocky reefs at depths less than 6 m.

Etymology. The specific epithet, obscurus, is Latin for dark, in reference to the dark coloration of the holotype.

Nomenclatural discussion. Borodin (1927) based his description of Exallias obscurus on a single specimen from Hawaii. Fowler (1927) referred to a previous report (Fowler 1923) of a Hawaiian specimen identified as Cirripectes alboapicalis and, believing these two species to be the same, placed Exallias obscurus in the synonymy of $\underline{C}$. alboapicalis. Borodin (1928) indicated several characters that differentiated the holotypes of the two species and recognized the Hawaiian form as a distinct species of the genus Cirripectes. Fowler (1928), without mentioning Borodin (1928), again placed E. obscurus in the synonymy of $\underline{\mathrm{C}}$. alboapicalis. Schultz (1941) and Chapman (1951) relegated both of the above names to the synonymy of $\underline{\mathrm{c}}$. variolosus. My findings support the conclusion of Strasburg (1956), who recognized all three of the above names as valiu.

Material examined. Types: AMNH 9363 (female: 116 m SL), holotype, Hawaiian Islands, Hawaii. 
Other material examined. Hawaiian Islands, Oahu: ANSP 83819 (1: 36), BPBM UNCAT. (4: 33-107; 19 MAR 1950), BPBM UNCAT. (24:62-126; 6 OCT 1951), BPBM UNCAT. (15:61-132; 10 SEP 1951), BPBM 4912 (1), BPBM 10035 (2: 54, 55), BPBM 19581 (12:58-134), ROM UNCAT. (1), USNM 228158 $(1: 86)$.

\section{Cirripectes perustus Smith}

Cirripectus perustus Smith, 1959:238 (Malindi).

Diagnosis. A species of Cirripectes with the dorsal fin entire and I, 3 pelvic-fin rays.

Description. Dorsal fin XI-XII (XII in $98.7 \%$ of specimens), 14-15 ( 14 in $98.7 \%$ ); anal fin II, 14-16 (15 in 96.2\%); total procurrent caudal-fin rays 10-12; pelvic fin $I, 3$; vertebrae $10+20-21$ (20 in $97.4 \%)=30-31(30$ in $97.3 \%)$; last pleural ribs on vertebral centrum 11-12 (11 in 98.7\%); last epipleural rib on vertebral centrum 15-19 (15-18 in 97.4\%); anal pterygiophores $1-1-1,1-1-2$, or $1-2-1(1-1-2$ in $91.0 \%$ ); nuchal cirri 30-46 (34-42 in 90.6\%); supraorbital cirri 6-23 (12-21 in 90.5\%); nasal cirri 5-25 (8-17 in 90.5\%); LL tubes $0-3(0-2$ in $93.3 \%)$; last LI tube positioned at point between verticals from dorsal-fin ray 5-11 (6-8 in $89.8 \%)$; no scalelike flaps along LL; lower lip smooth mesially (plicate laterally); gill rakers 23-28 (based on 14 specimens); pseudobranchial filaments on one side 8-11 (based on 14 specimens); premaxillary teeth 239-258 (based on 3 specimens); dentary teeth 105-108 (based on 3 specimens); upper lip crenulae approximately 
36-48; nuchal cirri in 3-4 groups, 3-group condition results from fusion of bases of dorsalmost groups of cirri at apex of nape, 4-group condition frequently has dorsalmost groups meeting or overlapping at apex of nape (type B or D; Figure 7); first dorsal-fin spine of adults slightly or not longer than second $(0-1.5$ m longer in males, 0-1.0 mm longer in females); dorsal-fin membrane not incised above last dorsal-fin spine; dorsal-fin membrane attached to caudal fin in adults; cephalic pore system simple (1-2 pores at most positions; Figure 17); mid-snout pores present (Figure 17); extra interorbital pore position absent (Figure 17); two pore positions behind nuchal flap (Figure 17); male genital papilla with urogenital orifice located basally between two widely separated slender filaments (less than 1.0 long) on a fleshy swelling behind anus (type I; Figure 13); testes bulbous, length equals width; maximum SL about $82 \mathrm{~mm}$; ophioblennius larvae have not been examined.

The smallest mature female (ova about $0.4 \mathrm{~mm}$ diameter) examined was about 45 man SL. Males mature by about $35 \mathrm{~mm}$ SL.

Color in alcohol. Males (Figure 49) with uniformly chocolate-brown head, body, dorsal fin, and anal fin; caudal fin with pale triangular area posterodorsally, remainder of fin brown; pectoral and pelvic fins dusky; rugosities on anal-fin spines creamy white; all cirri dark brown.

Females (Figure 50) with dark-brown spots (about I diameter) on a brown background on head and body; narrow, diagonal, pale bar extending anteroventrally from nape, along posterior margin of orbit, onto upper lip; dorsal fin dark brown, with pale basal stripe extending 
longitudinally along fin; other colors as for males (where appropriate).

Color in life. Males (based on color slides of specimens from Chagos Archipelago) with head and anterior half of body yellow; posterior half of body reddish brown to brown; segmented dorsal fin, anal fin, and all but tips (which are orange) of uppermost caudal rays red; spinous dorsal fin yellow with tips of anterior spines orange; pectoral fins yellow, tips of about five ventralmost rays red; basal half of pelvic fins yellow, distal half red; rugosities on anal-fin spines red; nuchal and nasal cirri reddish brown; supraorbital cirri red; iris with yellow oval centered around pupil and oriented almost vertically, remainder of iris silvery white.

Female (based on a color slide of a specimen from Chagos Archipelago) similar to color in alcohol except: spots on body rusty brown; tips of dorsal elements reddish orange; caudal-fin rays light brown with reddish-orange cast; pectoral fin brown, tips of about five ventralmost rays ied; distal half of pelvic fin red; iris color same as for males.

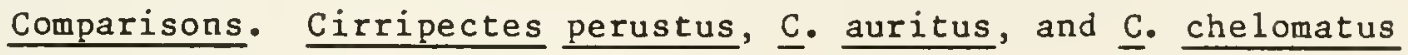
are the only Cirripectes species without the dorsal-fin membrane distinctly incised between spinous and segmented portions (it is

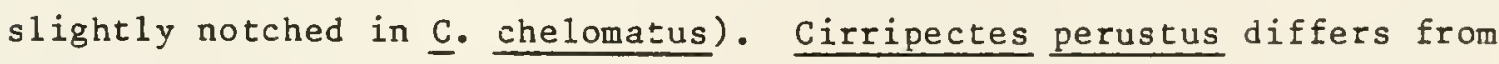
these species primarily in having $I, 3$ pelvic-fin elements (versus $I, 4$ ) and in the life color pattern.

Distribution. Cirripectes perustus is a widely distributed, although rarely collected, species occurring from Kenya and Madagascar 
eastward to Kiribati (Gilbert Islands). It has been collccted at depths ranging from 0 to about $25 \mathrm{~m}$.

Etymology. Smith (1959) did not provide the etymology for the specific name, but it is probably a combination of the Latin per, meaning very, and ustus, meaning burn. The name presumably refers to the bright red and yellow color of the male holotype and paratype.

Comments. Although Cirripectes perustus is widely distributed, I have found no geographic variation across its range, and very little variation within populations, in the characters examined.

Randall (1955) reported four specimeus identified by Strasburg as Cirripectes sp. From Onotoa. It was noted that this form was probably undescribed and was distinctive in lacking a notch between the spinous and segmented dorsal-fin elements. Although Randall (1955) stated that. Strasburg was working on these specimens, Strasburg did not publish a description and the species was subsequently described by Smith (1959). Material examined. Types: RUSI 265 (male: 67 SL), holotype, Malindi, inenya, 1952; RUSI 517 (male: 70), paratype, Malindi, Kenya. Other material examined. Kenya: LACM 30859-18 (1: 58). Madagascar: MNHN (3 specimens collected by L. A. Maugé). Seychelles Islands, Cosmoledo Group: AMSP 147667 (3: 29-71). Seychelles Islands, Farquhar Group: MNHN 54-33 (1). Chagos Archipelago: ROM 46587 (1: 52 ), ROM 46588 (1: 57), ROM 46589 (14:23-39), ROM 46590 (16: 21-35), ROM 46591 (1: 47), ROM 46592 (2:61, 62), ROM 46593 (1: 57), ROM 46594 (3: 57-60), ROM 46595 (2: 55, 61), ROM 46596 (1: 35). Nicobar Islands: SMF 17079 (2: 32-44), SMF 17080 (1: 54). Philippine Islands: USNM $228268(2: 20,30)$, USNM 228270 ( $1: 69)$, USNM 229270 ( 1 : 
59), USNM $228272(2: 56,62)$. Taiwan: NTUM 5791 (1: 82). Papua-New Guinea: USNM 228271 (1: 34). Caroline Islands: CAS UNCAT. (1: 63; GVF 1912), CAS UNCAT. ( 1 : 47; RAYANGEL ATOLL, 26 AUG 1956), CAS UNCAT. (2: 56-63; KAYANGEL ATOLL, 8 OCT 1956), CAS UNCAT. ( $1: 35$; SOROL ATOLL, GVF 993), CAS $38843(3: 37-45)$, CAS 38847 (1: 47), CAS 48096 (2: 37 , 39), CAS $48942(1: 52), \operatorname{CAS} 48946(1: 64), \operatorname{CAS} 48961(2: 38,58)$, CAS 48971 (3: 51-55). Gilbert Islands: BPBM UNCAT. (2: 61, 64), BPBM UNCAT. ( $2: 29,43)$.

\section{Cirripectes polyzona (Bleeker)}

Salarias (Cirripectes) polyzona Bleeker, 1868:278 (Amboina). Blennius canescens Garman, 1903:236 (eastern entrance of Mbengha Passage, Fiji Islands).

Diagnosis. A species of Cirripectes with a complex cephalic seusory pore system, I, 3 pelvic fin rays, and one pore posterior to the ventral edge of the ventralmost group of nuchal cirri.

Description. Dorsal fin XI-XIII (XII in $99.2 \%$ of specimens), 13-15 (14 in $96.2 \%)$; anal fin II, 14-16 (15 in 97.0\%); total procurrent caudal-fin rays 9-12; pelvic fin 1,3 ; vertebrae 10 ( 1 with 9) $+19-20$ $(20$ in $98.2 \%)=29-31(30$ in $98.0 \%)$; 1ast pleural ribs on vertebra1 centrum 11; last epipleural rib on vertebral centrum 15-19 (16-18 in $96.9 \%)$; anal pterygiophores $1-1-1,1-1-2$, or $1-2-1$ ( $1-1-2$ in $75.4 \%)$; nuchal cirri $32-44(37-41$ in $73.7 \%)$; supraorbital cirri $4-15$ (6-11 in $85.9 \%)$; nasal cirri $5-24$ ( $8-14$ in $75.0 \%)$; LL tubes $2-9$ ( $4-7$ in $77.6 \%$ ); 
last II tube positioned at point between verticals from dorsal-fin ray 7 and caudal-fin base (8-13 in $86.7 \%)$; no scalelike flaps along LL; lower lip smooth mesially (plicate laterally); gill rakers 18-25 (based on 27 specimens); pseudobranchial filaments on one side 7-10 (based on 27 specimens); premaxillary teeth 170-191 (based on 8 specimens); dentary teeth 79-100 (based on 8 specimens); upper lip crenulae approximately 32-45; nuchal cirri in 4 groups with slightly expanded nuchal flap (type B; Figure 7); first dorsal-fin spine of adults of both sexes equal to or slightly $(0-3.0 \mathrm{~mm})$ longer than second; dorsal-fin membrane deeply incised above last dorsal-fin spine; dorsal-fin membrane attached to caudal fin in adults; cephalic pore system complex (more than 3 pores at most positions; Figure 17; number of pores increases with increasing SL); mid-snout pores present (Figure 17 ); extra interorbital pore position present (Figure 17); pore positions behind nuchal flap 1 (Figure 17); male genital papilla with urogenital orifice located basally between two widely separated slender filaments (less than $1.0 \mathrm{~mm}$ long) on a fleshy swelling behind anus (type I; Figure 13); testes bulbous, width equals length; maximum SL about $100 \mathrm{~mm}$; ophioblennius larvae with 2 canines posteriorly on each dentary.

The smallest mature female (ova about 0.5 m diameter) examined was about $49 \mathrm{~mm}$ SL. Males mature by about $35 \mathrm{~mm}$ SL.

Color in alcohol. Ophioblennius stage larvae with body uniformly pale brown; nasal, orbital, and nuchal cirri browi to dark brown; blackish spot on each side of lower jaw above lower lip flap, another spot in groove behind posteriormost end of each maxillary and in groove above anterodorsal edge of maxillary. 
Young (18-45 SL; Figure 51) have stripe (about one and one-half pupil diameter in width) extending from above pectoral-fin base to end of caudal-fin rays, stripe frequently broken, appearing as string of beads with distinct, rounded spot above pectoral-fin base; area generally paler above stripe than below.

In sub-adults and adults (25-45 min SL), lateral body stripe breaks into about 12 brown bars (each about $3 / 4$ eye diameter); bar above pectoral-fin base frequently appears as blackish spot; small cream-colored spots on underside of head; upper lip with reticulated pattern of pale liues and spots on brown background; on upper half of cheek, reticulations develop into barred pattern extending onto nape (Figure 52).

Color in life. Striped phase (based on a specimen from Eniwetak Ato11) with pale-yellow spots on underside of head and on operculum, reddish-brown reticulations on snout, reddish-brown bars on upper cheek and nape; ventralmost nuchal cirri with narrow yellow-orange band basally; rest of cirri and their bases dark brown; spot above pectoral-fin base reddish brown; reddish-brown stripe beginning behind this spot and becoming black posteriorly; body tan above stripe and white to lavender below; iris with inner yellow ring and outer red ring; pectoral fin and upper caudal rays with yellowish tint. Adults (based on color slides of a male from Eniwetak Atoll and females from Enewetak, Tutuila, and Chagos Archipelago) similar to striped phase except reddish-brown reticuiations and bars on head more pronounced; pectoral-fin base with two reddish-brown bars; spot above pectoral-fin base and subsequent bars purplish black to dark brown; 
Enewetak male with yellowish interspaces between first 5 bars and yellow-tinted pectoral and pelvic fins. Females have pale-brown interspaces between dark-brown bars on body and red-tinted pectoral and pelvic fins.

Comparisons. Cirripectes polyzona can be differentiated from its congeners, except $\underline{C}$. perustus and $\underline{C}$. filamentosus, in having three pelvic-fin rays (versus 4). It is distinguished from $\underline{C}$. perustus and C. filamentosus by its complex pore pattern (versus simple), dark bars on body (versus uniformly brown or with dark spots), and one pore position behind nuchal flap (versus two). Cirripectes polyzona is most frequently confused with specimens of $\underline{C}$. castaneus because of their similar color patterns, but the latter has 4 segmented pelvic-fin rays and a simple cephalic pore pattern (Figure 17).

Distribution. Cirripectes polyzona is the most widely distributed species in the genus (Figure 23). It occurs from South Africa to the Gulf of Aden eastward to Johnston Atoll and the Line Islands, and from southern Honshu Island, Japan to the Capricorn-Bunker Group of islands, Great Barrier Reef, Australia.

Etymology. The specific epithet is derived from the Greek polys, meaning many, and the Latin zona, meaning belt. Presumably the name refers to the barred color pattern of adults.

Comments. No geographic variation was noted for the characters examined, but secondary sexual dichromatism was found. In life, an Enewetak Atoll male had yellow-tinted pectoral and pelvic fins, versus red tinted fins in females. Variation for this character is not known, as I have not seen life colors for males from other geographic areas. 
Nomenclatural discussion. Bleeker (1868) described Salarias (Cirripectes) polyzona based on two specimens from Amboina. The syntypic series (RMNH 4797) contains three specimens (two females and a badly damaged male). All three specimens are conspecific, but Bleeker (1868) appears to have excluded the damaged male from his description. Chapman (1951) and Schultz and Chapman (1960) erroneously referred $\underline{\text { S. }}$ (C.) polyzona to the synonymy of $\underline{C}$. sebae and gave the type locality of the latter as East Indies (type locality is unknown for $\underline{\mathrm{C}}$ sebae). The types of these two species are easily distinguished in that $\underline{C}$. polyzona has $I, 3$ pelvic-fin elements (versus 1,4 ) and multiple pores at most cephalic sensory pore positions (versus less than three). See the $\underline{C}$. castaneus account for a discussion of the nomenclatural status of $\underline{S}$. sebae. Schultz and Chapman (1960) were describing specimens of $\underline{C}$. polyzona from the Marshall Islands, not $\underline{\mathrm{C}}$. sebae.

Garman (1903) described Blennius canescens based on an ophioblennius stage larva from Fiji. As the holotype (MCZ 28294) is a specimen of Cirripectes polyzona, Blennius canescens is referred to the synonymy of $\underline{\text { C. polyzona. }}$

Material examined. Types: RMNH 4797 (3 specimens, 43-45 m $\mathrm{mL}$ ) syntypes of Salarias (Cirripectes) polyzona, East Indies, Aroe I., Amboina, collected by P. Bleeker; MCZ 28294 (1, 28) holotype of Blennius canescens, Fiji Islands, Mbengha Passage.

Other material examined. South Africa: BPBM 21772 ( $1: 29)$, RUSI 9141 (2). Mozambique: RUSI UNCAT. (2: 45, 50). Tanzania: USNM $227563(20: 26-52)$. Gulf of Aden: USNM 227560 (2:27, 45). Comoros Islands: CAS 33047 (4), CAS 40374 (1), CAS 40337 (2), CAS 40381 (6), 
USNM 22766? (4: 33-45). Seychelles, Cosmoledo Group: USNM 227666 (1), USNM 227684 (1). Seychelles, Amirante Isles: ANSP 126849 (2: 35-40), ANSP 147660 (1: 38). Agalega Is land: USNM 227564 (2 of 3 examined: 33, 36), USNM 227565 (1: 31). Chagos Archipelago: ROM 46598 (6), ROM 46599 (10), ROM 46600 (101), USNM 227562 (2:48, 53), USNM 227569 (19: 31-57), USNM 227690 (5: 42-56), USNM 227667 (1), USNM 227673 (2), USNM 227683 (5). Maldive Islands: SMF 13590 (3: 40-46). Sri Lanka: USNM 227566 ( 1 of 2 examined: 46), USNM 227567 (2: 59, 65). Nicobar Islands: SMF 17082 (1: 41), SMF 17083 (5: 29-47). Cocos (Keeling) Islands: ANSP $147661(2: 40,43)$, ANSP $147662(2: 34,35)$, ANSP 147663 (2: 34, 44), ANSP $147664(1: 23)$, ANSP $147665(1: 50)$. Christmas Island (Indian Ocean): WAM P.21811 (1:37), WAM P.26088-049 (1: 20). Viet Nam: CAS $48923(9: 34-51)$. Taiwan: BPBM UNCAT. (4), USNM 227656 (1: 62), USNM $227664(2: 51,52)$, USNM $227670(83: 23-69)$, USNM 227676 (6), USNM 227679 (7), USNM 227680 (15). Japan: FAKU 48270 (1: 20), FAKU $48273(1: 24)$, FAKU $4830(1: 23)$, FAKU $49174(2: 35,60)$, FAKU 111457 (1: 19). Philippine Islands: LACM UNCAT. (1), LACM 42479-20 (1), USNM 224945 (2:36-50), USNM 227620 (7:30-56), USNM 227621 (1: 43), USNM 227622 (8: $32-42)$, USNM 227671 (2), USNM 227674 (15), USNM 227677 (28), USNM 227682 (25), USNM 227686 (1), USNM 227687 (2), USNM 227688 (12). Indonesia: AMS I.19875-071 (90), BMNH 1858.4.21.83 (1), RMNH 20226 (4: 23-34), USNM 227624 (15: 17-55), USNM 227662 (5: 17-24). Netherland Indies: USNM 227618 (4: 43-49). Papua-New Guinea: USNM 227561 (2: 18, 19), USNM $227660(13: 25-44)$, USNM 227665 (1: 19). Louisiade Archipelago: USNM 227661 (5:41-48). Trobriand Islands: AMS I.17094-066 (15), BMNH 1974.5.25.3579-3582 (4), USNM 
$227559(150+: 18-50)$. Solomon Islands: ВРBM 5947 (1: 35), CAS 48966 (1). Australia, Queensland: AMS I.19473-099 (1: 58), AMS I. 19483-014 (9: 26-54), AMS I. 20770-100 (1: 41), AMS I.21422-145 (4: 32-51), US NM 227626 (6: 42-58), USNM 227689 (1). Mariana Islands: UF 31444 (1: 20). Caroline Islands: CAS 38822 (3), CAS 48898 (1), CAS 48909 (2), CAS 48924 (2), CAS 48934 (8), CAS 48952 (14), USNM 223369 (4: 37-47), USNM 227631 (13: 20-41). Marshall Islands: BPBM UNCAT. (17: 18-55), BPBM UNCAT. (1), BPBM 8173 (6), BPBM 15522 (2), GCRL 3984 (6), LACM 6674-100 (1), LACM 6674-101 (3), LACM 6679-38 (23), LACM 6679-39 (23), LACM $7279(1: 51)$, LACM 7422 (2: 53, 63), USNM 227657 (6: 44-59), USNM 227568 (4: 46-58), USNM 227669 (1). Gilbert Islands: AMS I.18045-23 (1), AMS I. 18052-022 (69), ВРВM 10645 (11), ВРBM 15320 (3), ВРВM 15361 (12). Rotuma Island: USNM 227625 (1: 47). Fiji Islands: USNM UNCAT. (2), USNM 227675 (1). Tonga Islands: USNM 227623 (29: 27-59). S amoa Islands: BPBM 11311 (2), UF 31445 (10), UF 31449 (18), USMM 52294 (1: 37), USNM $126227(2: 43,46)$. Phoenix Islands: USNM 227627 (1: 46), USNM $227658(2: 19,19)$, USNM 227668 (1), USNM 227681 (3). Howland Island: USNM 227678 (3). Baker Island: USNM 227672 (1). Iine Islands: ANSP $64316(1: 41)$, ANSP 64321 (1: 38), BPBM UNCAT (6), BPBM UNCAT. (3), BPBM 15372 (20), BPBM 25204 (14), CAS UNCAT. (16: 21-45), USNM 227685 (5). Johnston Atoll: USNM 227551 (20: 26-54).

\section{Cirripectes quagga (Fowler and Ball)}

Rupiscartes quagga Fowler and Bal1, 1924:273 (Wake Island). Cirripectus Iineopunctatus Strasburg, 1956:248 (Oahu, Hawaii). 
Diagnosis. A species of Cirripectes with the following combination of characters: lower lip crenulate mesially, last pleural ribs on vertebral centrum 12,15 segmented dorsal-fin rays, 16 segmented anal-fin rays, 21 caudal vertebrae, and no scalelike flaps along anterior portion of lateral line.

Description. Dorsal fin XI-XIII (XII in $98.9 \%$ of specimens), 14-16 ( 15 in $92.5 \%$ ); anal fin II, 15-17 ( 16 in 90.0\%); total procurrent caudal-fin rays $10-15$ ( 1 with 15 ); pelvic fin 1,4 ; vertebrae $10+20-22$ $(21$ in $95.1 \%)=30-32(31$ in $95.1 \%)$; last pleural ribs on vertebral centrum 11-12 (12 in 91.5\%); last epipleural rib on vertebral centrum 19-24 (20-23 in $99.1 \%)$; anal pterygiophores $1-1-1,1-1-2$, or $1-2-1$ (1-1-2 in 84.8\%); nuchal cirri 23-36 (26-23 in 95.6\%); supraorbital cirri 2-15 (2-12 in 96.9\%); nasal cirri 6-16 (8-14 in 96.2\%); LL tubes 7-18 (10-18 in 94.5\%); last LL tube positioned at point between verticals from dorsal-fin ray 7 and caudal-fin base ( 11 to caudal-fin base in $94.8 \%$ ); no scalelike flaps along LL; lower lip crenulate mesially (plicate laterally); gill rakers 18-26 (based on 150 specimens); pseudobranchial filaments on one side 7-9 (based on 150 specimens); premaxillary teeth 152-190 (based on 26 specimens); dentary teeth 72-97 (based on 26 specimens); upper lip crenulae approximately 32-46; nuchal cirri in two groups separated ar mid-point of nape, no nuchal flap (type E; Figure 7); first dorsal-Fin spine of adults. longer than second (0.3-9.2 mm longer in males, 0-5.8 mm longer in females); dorsal-fin nembrane deeply incised above last dorsal-fin spine; dorsal-fin membrane attached to caudla peduncle in advance of caudal fin; cephalic pore system complex ( 3 or more pores at most positions; 
Figure 17; number of pores increases with increasing SL); mid-snout pores present (Figure 17); extra interorbital pore position present (Figure 17); two pore positions behind nucha! flap (Figure 17); male genital papilla with urogenital orifice located basally behind a single slender filament (less than $1.0 \mathrm{~m}$ long) on a fleshy swelling behind anus (type IV; Figure 13); testes elongate, width equals about half length; maximum SL about $74 \mathrm{~mm}$ ophioblennius larvae with two canines posteriorly on each dentary.

The smallest mature female (ova about $0.5 \mathrm{~mm}$ diameter) examined was about $34 \mathrm{~mm}$ SL. Males mature by about $35 \mathrm{~mm}$ SL.

Color in alcohol. Ophioblennius stage larvae (about 25-35 m SL) with pale-brown head and body; head with several short, wavy, brown spots on cheeks; about 3 brown bars between each orbit and upper 1ip; pupil-sized dark-brown spot on posterior part of lower lip on each side, and at symphyses of dentary bones and premaxillary bones, premaxillary symphyseal spot extending into vomerine region inside mouth; body with faint indications of brown bars; fins dusky; all cirri brown.

Juveniles and adults of both sexes show extreme variability in body color pattern, both within and among populations (Figures 53, 54, and 55). Head with pale- and dark-brown bars usually present, sometimes broken into spots; ocellated spot occasionally present on opercle; pattern on underside of head varying from uniformly brown to presence of one to three dark-brown bars with pale interspaces; posterior edge of branchiostegal membrane with pale margin, frequently preceded by a brown bar extending between ventral margins of opercles; body 
pigmentation variable: some specimens with uniformly dark-brown body; some with about 12-14 dark-brown bars with pale interspaces, each pale interspace frequently reduced to vertical row of small pale spots (about $1 \mathrm{~mm}$ diameter); others with pale area posteriorly on body from beneath dorsal-fin ray 11-13 to caudal-fin base, bordered anteriorly ly one or two broad dark-brown bars on dorsal half of body; dorsal fin with tips of anterior spines pale, remainder of fin brown; caudal fin with distal half of upper (and sometimes lower) rays pale, remainder of fin brown; anal fin dark brown; anal-fin spine rugosities pale to brown; pectoral fin with dark-brown band on tips of ventralmost rays, remainder dusky; pelvic fin dusky; nuchal cirri brown to black; nasal and supraorbital cirri brown.

Color in life. Adult coloration (based on color slides of specimens from Chagos Archipelago, Samoa, Marshall Islands, Hawaiian Islands, Marquesas Islands, and Henderson Island) is similar to that in alcohol except as follows: tips of anterior dorsal-fin spines and caudal-fin rays yellow to orange; pale (unfigmented) area on caudal peduncle of preserved specimens yellow or scarlet in 1 ife; Marquesan specimens with red bar on chin and short red bar on branchiostegal membranes immediately anterior to pelvic-fin bases; iris black with irregularly shaped yellow inner ring; supraorbital cirri yellowish brown; other cirri brown.

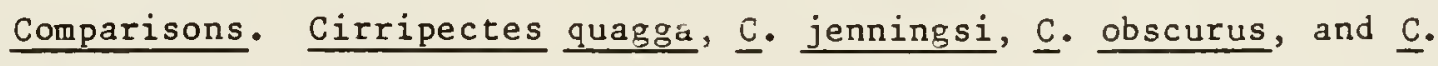
alboapicalis differ from all other Cirripectes in having lower 1 ip crenulate mesially, and last pleural ribs on vertebral centrum 12 . Cirripectes quagga differs from $\underline{C}$. obscurus and $\underline{C}$. alboapicalis in 
having 15 (versus 16) segmented dorsal-fin rays, 16 (versus 17) segmented anal-fin rays, 21 (versus 22) caudal vertebrae, and no scalelike flaps along anterior portion of lateral line (versus flaps present). Cirripectes quagga and $\underline{C}$. jenningsi are differentiated in the account of $\underline{\mathrm{C}}$. jenningsi.

Distribution. Cirripectes quagga is widely distributed throughout the Indian and Pacific oceans (Figure 24). In the Indian Ocean, it is known from South Africa to Tanzania eastward to Cocos (Keeling) Islands and northward to southern India and Sri Lanka. In the Pacific, it occurs from China to the Great Barrier Reef eastward to Henderson Island and the Hawaiian Islands. Although usually taken on reefs in water less than $10 \mathrm{~m}$ deep, $\underline{C}$. quagga has been collected at depths as great as $19 \mathrm{~m}$ in the Marquesas Islands.

Etymology. The specific epithet, quagga, is of Hottentot origin and refers to an extinct species of Equus, related to zebra, sometimes used to mean zebra (V. G. Springer, pers. comm). Fowler and Ball (1924) provided the following etynology: "Quagga, with reference to the rather obscure cross lines or bands."

Comments. Cirripectes quagga exhibits geographic variation in color pattern. The presence of a scarlet-colored caudal peduncle is known to occur only on Henderson Island females. The yellow-peduncle occurs on both males and females at numerous Pacific localities and Sri Lanka. The barred and/or spuited forms are the most frequently collected morphs and occur sympatrically with the other morphs. Although the barred and/or spotted versus colored-peduncle color morphs appear strikingly different in life, specimens taken at the same 
collecting station exhibit a broad range of color patterns intermediate between the colored-peduncle morphs and the barred and/or spotted morphs. As none of the oiner characters I examined show any differences correlated with a particular color morph, I believe the morphs reflect individual variants of a single highly variable species, C. quagga.

Nomenclatural discussion. Strasburg (1956) based his description of Cirripectes 1 ineopunctatus on a holotype, paratypes and non-types from the Hawaiian Islands, and ten non-type specimens from Jonnston Island. He differentiated $\underline{C}$. Iineopunctatus from $\underline{C}$. quagga on the basis of several color-pattern characters including presence of spots versus stripes, a minor tendency to have higher dorsal- and anal-ray counts, and a lower mean number of nuchal cirri. I have found specimens of $\underline{C}$. quagga with the same color-pattern characters as the Hawaiian specimens at numerous other localities. There are also many specimens of $\underline{\mathrm{C}}$. quagga with color patterns intermediate between the barred and spotted patterns. I found no differences in fin-ray or nuchal-cirri counts between the Hawaiian specimens and those from other localities. In the absence of any differences characteristic of the Hawaiian Islands specimens, I place $\underline{C}$. lineopunctatus in the synonymy of ‥ quagga.

Material examined. Types: ANSP 91013 (9 specimens: 33-47), paratypes of Rupiscarces quagga, Wake Island; ANSP 91191 (10: 33-51), paratypes of Rupiscartes quagga, same data as ANSP 91013; USNM 164198 (male: $58 \mathrm{~mm}$ SL), holotype of Cirripectus lineopunctatus, Hawaiian Islands, Oahu, Kaena Point; USNM 164199 (8: 28-69), paratypes of 
Cirripectus lineopunctatus, same data as holotype; USNM 164200 (6: 36-62), paratypes of Cirripectus 1 ineopunctatus, Hawaiian Islands, Oahu, Hanaumë Bay; USNM 164201 (4 of 5 examined: 28-44), paratypes of Cirripectus lineopunctatus, Hawaiian Is1ands, Oahu, Makapuu Point. Other material examined. South Africa: KUSI UNCAT. (1: 26; OUT OF RUSI 77-16), RUSI 77-20 (1: 45). Tanzania: USNM 227755 (1: 54). Comoros Islands: CAS $33249(1: 25)$, CAS $33427(5: 31-56)$, CAS 33768 (2: 31, 34). Cargados Carajos Shoals: USMM 227749 (1: 55). Agalega Islands: USNM 227752 (1: 38). Reunion Island: BPBM 20059 (3: 61-74). Chagos Archipelago: ROM 46608 (6), USNM 22/751 (1: 35), USNM $227756(4: 36-49)$, USNM 227760 (1:39), USNM $227761(2: 35,36)$, USNM $227767(16: 34-63)$. India: ANSP 146113(1:24). Sri Lanka: USM 227766 ( $1: 45)$. Cocos-Keeling Islands: ANSP $134746(2: 25,27)$, ANSP 134759 (5: 34-57). Indonesia: AMS I.19875-093 (1:44). China: CAS 48893 (1: 27). Taiwan: USNM 27754 (7:45-57). Australia, Queensland: AMS I.5023 (1: 49). Fiji Islands: ROM UNCAT. (31; NE 83-67). Mariana Isiands: CAS 38849 (10), CAS 38851 (1: 40), CAS 38852 (6), CAS 48911 (1). Caroline Islands: CAS UNCAT. (1: 40), CAS UNCAT. (8: 34-43; GVF 76), CAS 48905 (22: 26-56), CAS 48938(1: 49), CAS 48950 (1), CAS 48967 (16). Marshall Islands: BPBM 18413 (7 of 10: 36-54), GCRL 4389 (3), LACM 4033 ( $1: 47)$, LACM 6674-102 (3: 37-51), LACM 6679-40 (31), UF 9935 (3), USNM 227559 (2: 42-45), USNM 227762 (2: 38 , 42), USNM 22/763 (18: 36-64). Gilbert Islands: BPBM 15327 (2: 47, 53), USNM 167331 (4). Howland Island: USNM 227771 (291 34-71). Baker Island: USNM $227759(1: 42)$. Phoenix Islands: USNM UNCAT. (12 of $100+: 30-49 ; F-437)$, USNM $227632(1: 54)$, USNM $227750(2: 57,60)$, USNM 
227757 (19: 28-59), USNM 227768 (5: 28-31). Samoa Islands: UF UNCAT. (7 of 62: 28-43; DICK WASS), UF 31448 (4). Tonga Islands: USNM 227753 (7: 30-53). Cook Islands: CAS UNCAT. (5: 40-62). Society Islands: BPBM 10811 ( $1: 40)$, CAS UNCAT. (24: 28-55; GVF 21). Line Islands: BPBM UNCAT. ( $1 ; 21$ NOV 1953), USNM 227758 (1: 42), USNM 227765 (2 of 3: 38-57), USNM $227770(24: 31-74)$, USNM $227772(2: 36,55)$. Tuamotu Archipelago: BPBM UNCAT. (8: 38-55; 15 MARCH 1956), BPBM UNCAT. (27: 47-67; NO DATE), BPBM 14015 (8), CAS UNCAT. (2: 48, 49;9 JULY 1952), USNM UNCAT. (4; 15 MARCH 1956). Marquesas Islands: AMS I.22017-016 (10), BPBM UNCAT. (10: 26-55; 19 APRIL 1971;, BPBM UNCAT. (2: 30-53;17 JULY 1957), ВРBM $12103(1: 69)$, ВРBM $12111(15: 28-52)$, ВРВM 12144 (15: 25-61), ВРBM 12396 (28 of 29: 27-56), ВРBM 12586 (8: 37-51), ВРBM 12587 (2), BPBM 12654 (4). Henderson Island: BPBM 17070 (10: 29-46). Marcus Island: BPBM 7156 (24). Wake Is land: ANSP 65631-37 (7: 31-40), BPBM UNCAT. (28; JULY 1923), CAS 48970 (1). Johnston Island: ANSP 65598-602 (5: 39-52), BPBM 4924 (14), USNM 142115 (7), USNM 227550 (13: 31-63). Hawaiian Islands: BPBM 19580 (3).

One lot of 24 specimens (USMM 227764), cataloged as being from Aldabra Atoll, probably has erroneous collection data. These specimens were mixed with specimens of Cirripectes jenningsi and $\underline{\text { c. variolosus, }}$ which are otherwise known only from the Pacific Plate.

\section{Cirripectes randalli new species}

Diagnosis. A species of Cirripectes with the following combination of characters: complex cephalic sensory pore system, extra 
interorbital pore postion present (Figure 17), 36-42 nuchal cirri, last epipleural rib modally on vertebral centrum 19, body covered with small brown spots.

Description. Dorsal fin XII,14-15 ( 14 in 8 of 9 speicmens); anal fin II, 15-16 (15 in 8 of 9); total procurrent caudal-fin rays $10-12$; pelvic fin 1,4 ; vertebrae $10+20=30$; last pleural ribs on vertebral centrum 11; last epipleural rib on vertebral centrum 18-20; anal pterygiophores $1-1-1,1-1-2$, or $1-2-1$ (1-1-2 in 8 of 9 ); nuchal cirri 36-42; supraorbital cirri 12-26; nasal cirri 10-18; LL tubes 1-7; last LL tube on caudal-fin base; no scale:ike flaps along LL; lower lip smooth mesially (plicate laterally); gill rakers $26-27$ (based on 7 specimens); pseudobranchial filaments on one side 7-11 (based on 8 specimens); premaxillary teeth 200-213 (based on 2 specimens); dentary teeth 107-123 (based on 3 specimens); upper lip crenulae approximately 36-43; nuchal cirri in four groups with ventralmost group of cirri borne on a slightly expanded nuchal flap (type B; Figure 7); adults with first dorsal-fin spine slightly longer than second ( 4.6 longer in males, 0.5-2.0 longer in females); dorsal-fin membrane deeply incised above last dorsal-fin spine; dorsal-fin membrane attached to caudal fin in adults; cephalic pore system complex ( 6 or more pores at most positions; Figure 17; number of pores increases with increasing SL); mid-snout pores present (Figure 17); extra interorbital pore position absent (Figure 17); pore positions behind nuchal flap 2 (Figure 17); male genital papilla with urogenital orifice located basally between two widely separated slender.filaments (less than 1.0 mm long) on a fleshy swelling behind anus (type I; Figure 13); testes 
bulbous, length equals width; maximum SL abovt $107 \mathrm{~mm}$; ophioblennius larvae not known.

The smallest mature female (ova about 0.5 diameter) examined was about 85 SL. Ovaries were granular in 47 and 77 m SL females. Color in alcohol. A young female (47.4 SL) from Mauritius has pupil-sized dark-brown spots on a brown background over body; head with small pale spots on cheeks and snout, about 8 narrow pale bars on upper lip, background color brown; dorsal fin with pale triangular area distally on anterior spines, two lines of faint brown spots extending length of dorsal fin, one line of spots at base, another along middle of fin; caudal fin with pale triangular area distally on dorsalmost rays, remainder of fin dusky; anal fin with dark-brown sub-marginal line, remainder of fin dusky; pectoral and pelvic fins brown; nuchal cirri black; supraorbital and nasal cirri dark brown.

Adult females (Figure 56) have dense covering of pupil-sized dark-brown spots over body, overlaid on a background of about six to eight slightly irregular, broad, brown bars and pale interspaces (about half width of bars); spots extending onto basal half of all fins; background color of head brown with faint brown diagonal bar extending from nape to posterior part of upper lip; head and throat covered with small dark-brown to pale spots (about half pupil diameter in width), 8-10 narrow bars (pale or dark brown) on upper 1ip; other colors as described for young female.

Adult male (based on a specimen from Cargados Larajos Shoals; Figure 57) similar to females except: body background color uniformly dark brown, spots not as on females; head dark brown with few scattered 
pale spots on cheeks and narrow pale hars on upper lip; body spots do not continue onto dorsal, caudal, and anal fins.

Color in life. Adult female (based on a color slide of a freshly dead Mauritius specimen) with reddish-brown spots on head; body spots dark brown; dorsal-fin rays with orange tips; tips of dorsalmost caudal-fin rays yellowish orange; supraorbital and nasal cirri yellowish brown; color of iris orange, but appears to be faded; other colors as described for females in alcohol.

Life colors of males unknown, but probably similar to those of the female described abo:s.

Comparisons. In the western Indian Ocean, the color pattern of Cirripectes randalli resembles that of female $\underline{C}$. gilberti and female $\underline{C}$. perustus. Cirripectes perustus differs from $\underline{C}$. gilberti and $\underline{C}$. randalli in having pelvic-fin rays $I, 3$ and a simple cephalic sensory pore system. Cirripectes randalli differs from $\underline{\text { C. gilberti }}$ in lacking an interorbital pore postion (present in $\underline{C}$. gilberti) and in having 36-42 nuchal cirri (versus 50-59). Cirripectes randalli is also similar to the Western Australian C. hutchinsi, but differs in that

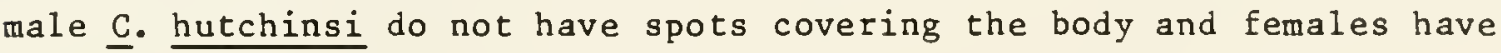
spots restricted to the ventral half of the head and, usually, midlateral region of body. There is also a modal difference in the position of the last epipleural rib, on vertebral centrum 19 in $\underline{C}$. randalli and 18 in C. hutchinsi, but the range of variation for this character in the latter species includes that of the former. These two species also have allopatric distributions, with $\underline{\mathrm{C}}$. hutchinsi known only from the southeastern Indian Ocean. 
Distribution. Cirripectes randalli is known only from Mauritius and Cargados Carajos Shoals in the western Indian Ocean (Figure 22 ). of the nine known specimens of this species, the eight females were collected at Mauritius and the male at Carados Carajos Shoals. It is known to inhabit rocky shores at depths of 0-1 m at Mauritius and depths as great as $7.5 \mathrm{~m}$ at Cargados Carajos Shoals.

Etymology. This species is named for Dr. John E. Randall in recognition of his many contributions to tropical marine ichthyology.

Material examined. Holotype: USNM 227873 (male: 92 um SL), Cargados Carajos Shoal, $16^{\circ} 26^{\prime} \mathrm{S}, 059^{\circ} 36^{\prime} \mathrm{E}$, coral patch in surge channel about 100 yds. off Raphael on west side, fossil coral rock patch with young corals, vertical undercut, many small caves - blind surge channel, 0-25 ft. (0-7.6 m).

Paratypes: RUSI 74-99 (1: 47), Mauritius, La Preneuse, surge channel, 8 Mar. 1971; RUSI 22306 (1: 79), Mauritius, collection date unknown; BPBM 20165 (6: 77-107), Mauritius, Trou d'Eau Douce and Palmar, rocky shore, light surf, 0-1 m.

\section{Cirripectes springeri new species}

Diagnosis. A species of Cirripectes with the following combination of characters: no mid-snout pores, type I male genital papilla (Figure 13), 31 vertebrae, and small spots on the body.

Description. Dorsal fin XII-XIII (XII in $97.8 \%$ of specimens),14-16 (15 in 95.7\%); anal fin II, 15-17 (16 in 93.6\%); total procurrent caudal-fin rays 10-12 (one specimen has 13); pelvic fin I, 4; vertebrae 
$10-11(10$ in $97.8 \%)+20-21(21$ in $93.3 \%)=30-31(31$ in $95.6 \%)$; last pleural ribs on vertebral centrum 11; last epipleural rib on vertebral centrum 16-19 (17-18 in 90.9\%); anal pterygiophores $1-1-1,1-1-2$, or $1-2-1(1-1-2$ in $84.4 \%)$; nuchal cirri $27-38$ (29-36 in $93.3 \%)$; supraorbital cirri $2-9(5-7$ in $84.7 \%)$; nasal cirri $5-23(6-23$ in 97. $9 \%)$; LL tubes $0-5(1-5$ in $97.0 \%)$; last Lu tube positioned at point between verticals from dorsal-fin ray $2-8$ (3-6 in $88.1 \%$ ); no scalelike flaps along LL; lower lip smooth mesially (plicate laterally); gill rakers 23-30 (based on 46 specimens); pseudobranchial filaments on one side 7-8 (based on 46 specimens); premaxillary teeth 187-227 (based on 11 specimens); dentary teeth 91-122 (based on 11 specimens); upper 1ip crenulae approximately 36-47; nuchal cirri in four groups with a small nuchal flap bearing ventralmost cirri (type B; Figure 7); first dorsal-fin spine of adults longer than second (4-20 mm longer in males, 2-7 m longer in females); dorsal-fin membrane deeply incised above last dorsal-fin spine; dorsal-fin membrane attached to caudal fin in adults; cephalic pore system simple (1-2 pores at most positions; Figure 17); mid-snout pores absent (Figure 17); extra interorbital pore position absent (Figure 17); two pore positions behind nuchal flap (Figure 17); male genital papilla with urogenital orifice located basally between two widely separated slender filaments (less than 1.0 um long) on a fleshy swelling behind anus (type I; Figure 13; one aberrant specimen with 3 filaments); testes bulbous, length equals width; maximum SL about 70 unin; ophioblennius larvae with two canines posteriorly on each dentary. 
The smallest mature female (ova about $0.4 \mathrm{~mm}$ diameter) examined was about $40 \mathrm{~mm}$ SL. Males mature by about 40 min SL.

Color in alcohol. Ophioblennius stage larvae (based on a $17.9 \mathrm{~mm}$ SL larva from Great Banda Island) with uniformly brown head and body; fins dusky; nuchal and supraorbital cirri dark brown; nasal cirri brown.

Juveniles, subadults, and adults of both sexes with chocolate-brown head and body covered with spots (0.5-1.0 miameter), spots on head and anterior quarter of body pale, becoming dark brown posteriorly; dorsal fin dark brown, all but posteriormost $4-6$ rays with pale tips; caudal fin dark brown with translucent triangular area distally on dorsalmost 3-4 rays; anal and pelvic fins dark brown; pectoral fin dark brown with small pale spots on basal quarter of fin (Figure 58).

Color in life. Adults (based on a color slide of a freshly dead adult male from Sumilon Island, Philippines) with orangish-red spots on anterior half of $\mathrm{fish}$ and basally on pectoral fin; tips of dorsal-fin elements and upper caudal-fin rays orangish red; iris red; other colors as in alcohol.

Comparisons. Cirripectes springeri, $\underline{C}$. chelomatus, and $\underline{\mathrm{C}}$. Eilamentosus are the only Cirripectes species that do not have mid-snout pores as adults. Cirripectes filamentosus and C. chelomatus both differ from $\underline{\text { C. }}$ springeri in having a type II male genital papilla (versus type I), and 30 vertebrae (versus 31 ). Cirripectes springeri and $\underline{C}$. chelomatus have allopatric geographic distributions.

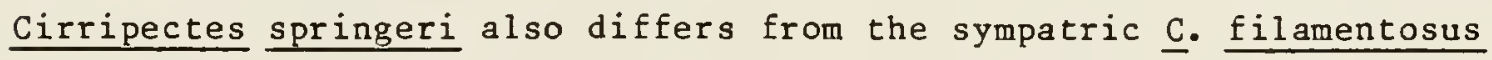
in having spots on the body (versus uniformly brown body, rarely with a few pale spots or bãz above pectoral-fin base). 
Distribution. Cirripectes springeri is known to occur in the Solomon Islands, Bismarck Archipelago, Ninigo Islands, Moluccas, and Philippines (Figure 26). It has been collected at depths as great as $18 \mathrm{~m}$ in the Ninigo Islands, but most specimens were collected from water shallower than $10 \mathrm{~m}$.

Etymology. Cirripectes springeri is named after Victor G. Springer, in recognition of his contributions to blennioid systematics. Material examined. Holotype: BPBM 22121 (male: 54 mm SL), Philippine Islands, Sumilon Island, west side, outer reef flat on live coral, $2 \mathrm{~m}, 25$ Aug. 1981 .

Paratypes: AMS I.18469-174 (1: 56), Indonesia, Ceram, Marsegoe Bay, $03^{\circ} 00^{\prime} 30^{\prime \prime} \mathrm{S}, 128^{\circ} 03^{\prime} 18^{\prime \prime} \mathrm{E}, 0-8 \mathrm{~m}, 1$ April 1975; CAS 48956 (2: 37, 49), Solomon Islands, New Georgia, outer reef of Wana-Wana Island, Blackett St., 25 June 1944; USNM 210166 (1: 20), Molluccas Islands, Ceram, Tandjun Liang, east shore, Piru Bay, 0-1 m, $10 \mathrm{Jan}$. 1973; USNM 227617 (1: 51), Netherlands Indies, collection date unknown; USNM 228283 (1: 43), Bismarck Archipelago, New Britain, $04^{\circ} 14^{\prime} 05^{\prime \prime}$ $\mathrm{S}, 152^{\circ} 10^{\prime} 25^{\prime \prime} \mathrm{E}$, Dawapia Rocks (Beehives) near entrance to Simpson Harbor, Rabaul, 27 Feb. 1965; USNM 228284 (2: 22, 39), Philippine Islands, Southern Negros Oriental, Port Siyt, $09^{\circ} 04^{\prime} \mathrm{N}, 123^{\circ} 10^{\prime}$ 48" E, 0-3.1 m, 28 April 1979; USNM 228285 (7: 18-60), íoluccas, Banda Island, Indonesia, $04^{\circ} 32^{\prime} 50^{\prime \prime} \mathrm{S}, 129^{\circ} 54^{\prime} \mathrm{E}$, north shore of Great Banda, island directly south of Naira Island, 0-10 ft (0-3 m). 8 Mar. 1974; USNM 278286 (1: 42), Papua-New Guinea, Umboi Island, in lee of Higgins Point, $06^{\circ} 41^{\prime} 54^{\prime \prime} \mathrm{S}, 147^{\circ} 53^{\prime} 06^{\prime \prime} \mathrm{E}, 0-4.5 \mathrm{~m}, 18$ June 1979 ; USNM 228287 (8: 8), Phillipine Islands, Siquijor Island, near Tonga 
Point, $09^{\circ} 13^{\prime} 30^{\prime \prime} \mathrm{N}, 123^{\circ} 28^{\prime} \mathrm{E}, 0-1.2 \mathrm{~m}, 15 \mathrm{Mar} .1979$; US NM 228288 ( $1: 30$ ), Phillipine Islands, west side of Solino (Selinog) Island, Zamboanga del Norte, Mindanao, $08^{\circ} 51^{\prime} 24^{\prime \prime} \mathrm{N}, 123^{\circ} 24^{\prime} 36^{\prime \prime}$ E, 0-4.5 m, 3 May 1979; USNM 228289 (1: 32), Papua-New Guinea, Madang Harbor, south end of Massas Island, 5-15 ft (1.5-4.5 m), 1 June 1970; USNM 228290 (2: 44, 53), Papua-New Guinea, Madang Harbor, off southern edge of Massas Island, 10-45 ft (3-13.7 m), 31 May 1970; USNM 228292 (6: 45-54), Papua-New Guinea, Madang Harbor, off southern edge of Massas Is 1 and, 35-45 ft $(10.7-13.7 \mathrm{~m}), 26$ May 1970; US NM 228293 (1: 26), Papua-New Guinea, Kranket Island, lagoon on northwest side, $05^{\circ}$ $11^{\prime} 30^{\prime \prime} \mathrm{S}, 145^{\circ} 50^{\prime} 42^{\prime \prime} \mathrm{E}, 0-0.9 \mathrm{~m}, 7$ Nov. 1978; USNM 228294 (10: 23-49), Papua-New Guinea, Madang Harbor, southern tip, $05^{\circ} 10^{\prime} 18^{\prime \prime} \mathrm{s}$, $145^{\circ} 51^{\prime} 24^{\prime \prime} \mathrm{E}, 0-18.3 \mathrm{~m}, 6$ Nov 1978; USNM 228297 (1: 57), Papua-New Guinea, Ninigo Islands, southeast of Ami Island, $01^{\circ} 14^{\prime} \mathrm{S}, 144^{\circ}$ $22^{\prime}$ E, 0-6 m, 22 oct. 1978.

\section{Cirripectes stigmaticus Strasburg and Schultz}

Cirripectes stigmaticus Strasburg and Schultz, 1953:132 (Rongerik Atoll, Marshall Islands).

Cirripectes cruentus Smith, 1959:238 (Pinda).

Diagnosis. A species of Cirripectes with the follcring combination of characters: simple cephalic sensory pore system, no extra interorbital pore position (Figure 17), 15 segmented dorsal-fin rays, 16 segmented anal-fin rays, 21 caudal vertebrae, and one pore behind the vertralmost group of nuchal cirri on each side. 
Description. Dorsal fin XI-XIII (XII in $98.6 \%$ of specimens), 14-16 ( 15 in $95.3 \%$ ); anal fin II, 15-17 (16 in 96.2\%); total procurrent caudal-fin rays $10-14$; pelvic fin $I, 4$; vertebrae $10+20-22$ (21 in $94.6 \%)=30-32(31$ in $94.6 \%)$; last pleural ribs on vertebral centrum 11; last epipleural rib on vertebral centrum $17-21$ (18-19 in $87.0 \%)$; anal pterygiophores $1-1-1,1-1-2$, and $1-2-1$ ( $1-1-2$ in $86.1 \%$ ); nuchal cirri $34-47(34-40$ in $87.2 \%)$; supraorbital cirri $7-22$ (7-14 in $81.4 \%)$; nasal cirri $8-29(10-21$ in $76.7 \%)$; LL tubes $1-10(3-8$ in $89.7 \%)$; last LL tube positioned at point between verticals from dorsal-fin ray 7 and caudal-fin base ( $9-15$ in $81.4 \%$ ); no scalelike flaps along LL; lower lip smooth mesially (plicate laterally); gill rakers 23-30 (based on 34 specimens); pseudobranchial filaments on one side 7-12 (based on 34 specimens); premaxillary teeth 221-248 (based on 3 specimens); dentary teeth 114-123 (based on 3 specimens); upper lip crenulae approximately 35-52; nuchal cirri in four groups, ventralmost group of cirri on each side borne on a slightly expanded nuchal flap (type B; Figure 7); first dorsal-fin spine of adults slightly longer than secnnd $(0.2-12.0$ man longer in males, 0-2.2 longer in females); dorsal-fin membrane deeply incised above last dorsal-fin spine; dorsal-fin membrane attached to caudal fin in adults; cephalic pore system simple (1-3 pores at most positions; Figure 17); mici-snout pores present (Figure 17); extra interorbital pore position atsent (Figure 17); pore positions behind nuchal flap 1 (Figure 17); mele genital papilla with urogenital orifice located basally between two widely separated slender filaments (less than 1.0 long) on a Eleshy swelling behind anus (type I; Figure 13); testes bulbous, length equals width; maximum SL 
about 100 ; ophioblennius larvae with two canines posteriorly on each dentary.

The smallest mature female (ova abcut 0.5 miameter) examined was about $45 \mathrm{~mL}$ SL. Males mature by about $35 \mathrm{~mm}$ SL.

Color in alcohol. Small ophioblennius stage larvae (based on an $18.8 \mathrm{~mm}$ SL specimen from the Gilbert Islands) with dark-brown pigment spot on each side of lower lip above small lip flap; pale bar extending from midventral margin of orbit to and over posterior fourth of upper lip on each side; tip of snout densely covered with melanophores; remainder of head and body with uniformly distributed melanophores.

Large ophioblennius stage larvae (based on a $24.6 \mathrm{~mm}$ SL specimen from Tanzania) with 3-4 faint brown bars on head and about 11-12 faint brown bars on body (brown bars are narrower than cream-colored interspaces); other colors same as smaller larva.

Juvenile and adult females (Figure 59) with a reticulated pattern of pale spots enclosed by wavy brown lines over head and anterior half of body; posterior half of body with dark-brown spots, sometimes fusing into irregular bars, on a paler-brown background; pectoral-fin base and proximal ends of rays with pale spots on a brown background; transluscent triangular area distally on anterior dorsal-fin spines and dorsalmost caudal-fin rays, otheiwise, fins dusky; nasal and supraorital cirri dark brown; nüchal cirri black.

Juvenile and adult males (Figure 60! similar to females except as follows: body from beneath middle of spinous-dorsal fin to end of dorsal-fin base with small white spots (about 1.0 miameter) that frequently fuse into irregular wavy bars; rugosities on anal-fin spines dark brown. 
Color in life. Females (based on color slides of living and freshly dead specimens from Fiji and Queensland, Australia) with head and anterior half of body with rust.-colored reticulations enclosing pale-olive spots; at about mid body, spots becoming larger and less defined, and reticulations beginning to break apart; near caudal-fin base, reticulations reduced to several brown spots and short wavy lines on yellowish-brown background; tips of dorsal-fin spines and rays yellowish orange, most elements with red line on basal third, remainder of fin yellowish brown; caudal fin yellow basally with dorsalmost and ventralmost rays orange and distal half of other rays yellowish orangc; anal fin dusky brown with two longitudinal red lines, one located outward from fin base at distance of about one-third and other at about two-thirds length of each element; nuchal cirri black with yellow bar basally on each cirrus in ventralmost group; iris with narrow yellow ring around pupil, irregular red ring around outer edge, and black area between them; other colors as in alcohol.

Males (based on color slides of froshly dead specimens from Queensland, Australia, New Caledonia, Fiji, Samoa, and the Gilbert Islands) with dark-brown background color on head and body; reddish-brown reticulations overlaid on head and anterior half of body; posterior half of body with scarlet spots and wavy 1 ines; tips of anterior dorsal-fin spines yellowish orange; scarlet spots and lines of body extending onto basal fourth of segmented-dorsal fin, above these, rays scarlet, becoming into yellowish orange at tips, membranes brown; tips of drosal and ventral caudal-fin rays yellow to orange, otherwise, fin scarlet; anal fin with scarlet spots basally, orange line 
submarginally, remainder brown; body reticulations extending onto basal third of pectoral-fin rays, tips of lower rays orange, upper rays yellow, membranes dusky ventrally and transluscent dorsally; pelvic fins brown with scarlet line submarginally; anal-fin spine rugosities brown; other colors as described for female.

The Gilbert Islands male differs from the other males as follows: darker overall color; reticulations on head enclose pale spots; fins tend to have brown lines and spots instead of scarlet; and iris is almost entirely yellow.

Comparisons. On the Pacific plate, Cirripectes stigmaticur is easily distinguished from other Cirripectes species by its color pattern. It further differs from all plate species except $\underline{C}$. auritus in having a simple cephalic sensory pore pattern, and lacking an interorbital pore position (Figure 17). Cirripectes auritus has a broadly expanded nuchal flap (type A; Figure 7) absent in other plate species.

of those Cirripectes species whose geographic distribution does not include the Pacific plate, $\underline{C}$. stigmaticus differs from all except $\underline{C}$. $\underline{\text { filamentosus, }} \underline{\mathrm{C}}$. chelomatus, and $\underline{\mathrm{C}}$ springeri in usually having 15 (versus 14) segmented dorsal-iin rays and 16 (versus 15) segmented anal-fin rays. In addition, only $\underline{C}$. stigmaticus and $\underline{\mathrm{C}}$. springeri typically have 21 caudal vertebrae. Cirripectes stigmaticus differs

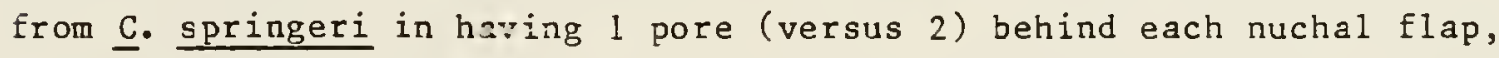
and mid-snout pores present (versus absent).

Distribution. Cirripectes stigmaticus is a widely distributed species occurring from Mozambique to Kenya on the African coast, 
throughout the Indian Ocean and western Central Pacific, to the Marshall and Samoa Islands (Figure 25). It is typically found on rocky and coralline structures, usually at depths less than 10-15 $\mathrm{m}$, but occasionally as deep as $20 \mathrm{~m}$.

Etymology. The specific epithet is derived from the Greek stigma, meaning spot or mark, and refers to the body markings.

Comments. Apart from sexual dichromatism and slight color differences noted for the Gilbert Islands male, no geographic or sexual variation was observed for the characters examined.

Nomenclatural discussion. Smith (1959) described Firripectes cruentus based on one specimen from Pinda, Mozambique. The only comparison he made was that it "differs from other species in markings." Smith was aware of the description of Cirripectes stigmaticus by Strasburg and Schultz (1953), as he cited the paper on the same page as his description of $\underline{C}$. cruentus, but he did not comment on the similarity of the markings on his specimen to those described and figured for $\underline{\text { C. stignaticus. }}$

Material examined. Types: RUSI 246 (male: 63 SL), holotype of Cirripectus cruentus, Baixo Pinda, Mozambique (radiograph, counts, and measurements provided by P. C. Heemstra); USNM 164962 (male: 64), holotype of Cirripectes stigmaticus, Marshall Islands, Rongerik Atoll, Latoback Islet, lagoon reef; and the following paratypes of Cirripectes stigmaticus: UsiNM 142129 (1: 84), Marshall Islands; USM 154673 (2: 45, 57), Marshall Islands; USNM 154674 (3: 68-83), Marshall Islands; USNM 164961 (16: 36-79), Gilbert Islands; USNM 164963 (2: 62, 67), Marshal1 Islands; USNM 164964 (2: 89, 100), Marshall Islands; USNM 
Other material examined. Mozambique: RUSI UNCAT. (1: 24).

Madagascar: MNHN (12 specimens collected by A. Mauge). Mauritius: RUSI 74-309: 1 ), USNM 227701 ( $1: 78$ ), USNM $227723(3: 39-87)$, USNM 227725 (4: 66-87). Cargados Carajos Shoals (St. Brandons Shoals): USNM $227711(3: 22-83)$, USNM $227721(1: 98)$, USNM 227713(1: 69). Comoros Islands: CAS 40380 (3), USNM 215213 (5), USNM 227714 (4: 50-84). Tanzania: RUSI UNCAT. ( $1: 24 ;$ OUT OF RUSI 14997). Renya : USNM $227874(1: 78)$. Seychelles Islands: USNM $227727(1: 43)$. Maldive Islands: BPBM 18912(1: 66). Sri Lanka: USNM 227701 (1: 70). Nicobar Islands: SMF 17085 (2: 42, 57). 3imilan Islands, Thailand: BPBM 22784 (1: 66). Christmas Island, Indian Ocean: WAM P.26083-039 (2: 29, 43). Indonesia: AMS I.18469-088 (3), AMS I.19875-092 (8). Australia, Western Australia: AMS I.21318-057 (1). Australia, Queensland: AMS I.19338-007 (1), AMS I.19454-027 (1), AMS I.19473-095 (14: 56-88), AMS I.19481-014 (6), AMS I.19483-016 (10), AMS I.20547-010 (4), AMS I.20755-035 (3), AMS I.20756-041 (5), AMS I.20757-006 (1), AMS I.20770-099 (1), AMS I.20774-077 (6), AMS I.21422-048 (3), USNM 227698 (1: 72), USNM 227709 (1: 74), Philippine Islands: USNM $227553(1: 25)$, USNM 227697 (9: 48-82), USNM 227700 (1: 40), USNM 227702 (1: 48). Indonesia: USNM $195699(2: 55,62)$, USNM 209727 ( $1: 51)$, USNM $227616(3: 36-55)$, USNM $227706(18: 19-54)$, USNM 227710 (13: 25-53), USNM 227731 (1: 58). Papua-New Guinea: USNM $227704(1: 35)$, JSNM $227705(2: 61,63)$, USNM $227707(1: 29)$, USNM $227708(1: 56)$, USNM 227720 (3: 46-53), USNM $227721(15: 21-61)$, USNM $227724(1: 20)$, USNM $227726(13: 32-59)$, USNM 228280 (1: 54). Bismarck Archipelago: USNM 227722 (4: 53-58). Solomon Islands: AMS 
I. 17486-029 (1), BPBM 5954 (1), BPBM 15611 (1), BPBM 16п35 (1), CAS 48959 (2). Vanuatu (New Hebrides): AMS I.17473-027 (2), BPBM UNCAT. (1: 73), CAS 48960 (2). Loyalty Islands: USNM 227699 (1: 79). New Caledonia: BPBM 11417 (1: 84). Fiji Islands: BMNH 1877.4.26.2 (1), ВРВM 14633 (1), ВPBM 20956 (3), USNM 227703 (2: 44-86), USNM 235728 (2), USNM 235732 (1), ZMB 9870 (1). Tonga Islands: USNM 227715 (1: 65). Caroline Islands: BPBM 7422 (1), BPBM 9230 (2), CAS 38810 (2), CAS 38811 (1), CAS 38812 (1), CAS 38813 (1), CAS 38817 (1), CAS 38844 (1), CAS 38870 (20), CAS 38872 (30), CAS 48897 (3), CAS 48945 (1), CAS 48947 (1), USNM 223457 (24: 41-74), US:01 $224387(11: 27-54)$, USNM 227628 (1: 48), . Kapingamarangi Atoll: CAS 48936 (3). Marshall Islands: BРBM 7998 (4), BPBM 18383 (2), LACM 4034 (1: 75). Gilbert Islands: AMS I.18047-012 (1), AMS I.18051-018 (1), AMS I.18052-047 (1), AMS I.18052-079 (1). Samoa Islands: ANSP 51009 (1:57), BPBM $11314(1: 46)$, UF 31450 (2).

\section{Cirripectes vanderbilti (Fowler)}

Ophioblennius vanderbilti Fowler, 1938:242 (Diamond Head, Oahu). Ophioblennius capillus Reid, 1943:381 (Honolulu, Diamond Head Light).

Diagnosis. A species of Cirripectes with the following combination of characters: 14 segmented dorsal-fin rays, 15 segmented anal-fin rays, smooth lower lip, white (red in life) spots and streaks un the snout and cheeks, nuchal cirri in two groups with base slightly swollen beneath ventralmost cirri on each side (Figure 7G), and male genital papilla with two closely appressed filaments (Figure 13B). 
Description. Dorsal fin XII,13-15 (14 in $94.0 \%$ of specimens); anal fin II, 15-16 (15 in 95.7\%); total procurrent caudal-fin rays $10-12$; pelvic fin $\mathrm{I}, 4$; vertebrae $10+20-21(20$ in $97.4 \%)=30-31$ (30 in 97.4\%); last pleural ribs on vertebral centrum 11 ; last epipleural rib on vertebral centrum 18-21 (19-20 in 93.0\%); anal pterygiophores 1-1-2 or $1-2-1$ (1-1-2 in $92.2 \%)$; nuchal cirri $31-42(35-40$ in $86.5 \%)$; supraorbital cirri 9-22 (10-15 in 78.9\%); nasal cirri 7-23 (8-15 in 91.7\%); LL tubes $0-5$; last LL tube or end of LL canal positioned at point between verticals from dorsal-fin ray 7 and caudal-fin base (8-14 in $94.4 \%$ ); no scalelike flaps $=1$ ung LL; lower lip smooth mesially (plicate laterally); gill rakers 23-30 (based on 21 specimens); pseudobranchial filaments on one side 8-9 (based on 21 specimens); premaxillary teeth 204-229 (based on 5 specimens); dentary teeth 86-108 (based on 5 specimens); upper lip crenulae approximately 42-54; nuchal cirri in two groups with a small nuchal flap bearing ventralmost cirri (type G; Figure 7); first dorsal-fin spine of adults slightly longer than second ( $1-5$ mm longer in males and females); dorsal-fin membrane deeply incised above last dorsal-fin spine; dorsal-fin membrane attached to caudal fin in adults; cephalic pore system complex ( 6 or more pores at most positions; Figure 17; number of pores increases with increasing SL;); mid-snout pores present (Figure 17); extra interorbital pore position present (Figure 17); two pore positions behind nuchal flap (Figure 17); male genital papilla with urogenital orifice located basally between two closely appressed slender filaments (less than $1.0 \mathrm{~mm}$ long) on a fleshy swelling behind anus (type II; Figure 13); testes bulbous, length equals width; maximum SL about 100 mm; ophioblennius larvae with two canines posteriorly on each dentary. 
The smallest mature female (ova about 0.6 m diameter) examined was about 42 SL. Males mature by about $35 \mathrm{~mm}$ SL.

Color in alcohol. Ophioblennius stage larvae have uniformly light-brown head and body, no distinct blackish spots, and all cirri dark brown.

Juveniles, subaduts, and adults of both sexes (Figure 61) have brown head and body; small (0.5-1.0 m diameter) pale spots on head, those on snout frequently joined to form narrow 1 ines running from margin of orbits onto upper lip; all cirri blackish; underside of head uniformly brown; fins bzuwn except for pale triangular area anterodorsally on spinous-dorsal fin and pale area over upper caudal-fin rays.

Color in life. Adults (based on color slide of 1 iving and photograph of freshly dead Hawaiian specimens) have brown body; head with small reddish-orange spots and streaks on brown background; all cirri black; underside of head pale brown; spinous-dorsal fin with spines red in translucent triangular area anterodorsally on fin, tips of first few segmented dorsal-fin rays red; tips of upper caudal rays red; anal-fin rays with whitish tips; otherwise, fins brown; iris black with thin yellow ring around pupil and bright reddish-orange ring around outer porcion.

Comparisons. Of the three Cirripectes species occurring in the Hawaiian Islands, $\underline{C}$. vanderbilti typically has 14 segmented dorsal-fin rays, whereas $\underline{\mathrm{C}}$. quagga has 15 and $\underline{\mathrm{C}}$. obscurus usually 16 . Cirripectes obscurus also has a pupil-sized black spot behind each eye, and usually has small white spots covering the head and about two-thirds of the 
body. Cirripectes quagga and $\underline{C}$. obscurus both have a crenulate lower lip (smooth mesially in $\underline{\text { C. vanderbilti). }}$.

$\underline{\text { Cirripectes vanderbilti }}$ and $\underline{C}$. variolosus occue sympatrically at Johnston Island, where they are the only species with white (red in life) spots and streaks on the snout and cheeks (see $\underline{C}$. variolosus account for differentiating characters).

Distribution. Cirripectes vanderbilti is restricted to the Hawaiian Islands and Johnston Island (Figure 26). It is usually found on coral reefs where it has been collected at depths ranging from near the surface to $10 \mathrm{~m}$.

Etymology. Fowler (1938) named this species after Mr. George Vanderbilt.

Nomenclatural discussion. Fowler (1938) based the description of Ophioblennius vanderbilti on 14 specimens, two from Oahu and 12 from Christmas Island, Line Islands. All these specimens are ophioblennius stage larvae of Cirripectes, but those from Christmas Island are $\underline{\text { C. }}$ variolosus. Fowler (1938) designated the Christmas Island specimens and one of the Oahu specimens paratypes, and the other Oahu specimen "type." This clearly links the name with the Oahu specimen designated "type." Fowler (1938) did not realize that his specimens were larvae and, in the same publication, identifieu adults of this species as $\underline{C}$. variolosus. Nevertheless, Ophioblennuis vanderbilti is the first name available for this Hawaiian Islands-Johnston Ialand endemic species.

Reid (1943) described Ophioblennius capillus based on one specimen from Diamond Head Light, Oahu. He stated that $\underline{0}$. capillus "differs from Ophioblennius vanderbilti Fowler in having only two canines in the 
upper jaw, more rays in the pectoral fins, nasal and orbital appendages, and a much deeper notch in the dorsal fin," Reid (1943) did not realize that his specimen (as woll as the others in the genus Ophioblennius as he defined it) was a larva. The canines at the tips of the jaws are gradually lost as the larvae metamorphose into the juvenile stage of development. The deeper dorsal-fin notch may reflect a torn membrane or a difference in methods of making the measurement. The difference in color of nasal and orbital appendages (cirri) could be an artifact of preservation. As for the difference in number of pectoral-fin rays, neither Fowler (1938) nor Reid (1943) provide this count for $\underline{0}$. vanderbilti, and I have found this character to have a constant mode of 15 for all Cirripectes species. As the types of $\underline{0}$. capillus and $\underline{0}$. vanderbilti represent the same species, the older name, O. vanderbilti, is considered the valid name.

Material examined. Types: USNM 120032 ( 1 specimen: 21 m SL), holotype of Ophioblennius capillus, Hawaiian Islands, Oahu, off Diamond Head, surface; ANSP 68432 (1: 22), holntype of Ophioblennius vanderbilti, Hawaiian Islands, Oahu, Diamond Head; ANSP 68433 (1: 22), paratype of Ophioblennius vanderbilti, Hawaiian Islands, Oahu, Diamond Head.

Other material examined. Hawaiian IsIands: ANSP 10493 (1: 58), USNM 78057 ( 1 ). Hawaiian Islands, Pearl and Hermes Reef: NMW 77809 (3: 31-37). Hawaiian Islands, Oahu: ANSP 83873 (3: 22-24), BPBM 4918 (1), BPBM 15212 (20), USNM 108846 (2: 23, 27), USNM 109320 (3: 37-62), US NM 205336 (4: 31-54), USNM 228162 (16:35-81), USNM 228163 (1: 66), USNM 258323 (2), USNM 258324 (5). Hawaiian Islands, Molokai: ANSP 
81990 (1: 34), BPBM 4917 (1). Hawaiian Islands, Maui: USNM 228161 (1: 54). Hawaiian Islands, Hawaii: USNM 160687 (7: 61-90). Johnston Island: BPBM UNCAT. (2; JULY 1923), BPBM 11041 (3), BPBM 15103 (65), BPBM 15360 (25), RUSI 74-23 (5), USNM 142114 (73: 35-61).

\section{Cirripectes variolosus (Valenciennes)}

Salarias variolosus Valenciennes in Cuvier and Valenciennes, 1836:317 (Guam).

Salarias nigripes Seale, 1901:127 (Guam).

Ophioblennius vanderbilti Fowler, 1938:242 (in part, only paratypes

from Christmas Island, Line Islands).

Ophioblenniuus sp. indet. Clark, 1938:185 (Nukuhiva Island).

Ophioblennius clarki Reid, 1943:380 (Nukuhiva Island).

Diagnosis. A species of Cirripectes with the following combination of characters: small white (red in 1ife) spots and/or narrow 1 ines on snout and around eye, nuchal cirri type B (Figure 7B), and male genital papilla type I (Figure 13A).

Description. Dorsal fin XI-XIII (XII in $99.2 \%$ of specimens), 13-15 ( 14 in $95.1 \%$ ); anal fin $11,14-16$ (15 in $95.5 \%)$; total procurrent caudal-fin rays $10-14$; pelvic fin $I, 4$; vertebrae 10 ( 1 with 9 ) + 19-21 $(20$ in $96.3 \%)=29-31(30$ in $96.5 \%)$; last pleural ribs on vertebral centrum 11; last epipleural rib on vertebral centrum 18-23 (19-21 in 93. $3 \%$ ); anal pterygiophores $1-1-1,1-1-2$, or $1-2-1$ (1-1-2 in $89.3 \%)$; nuchal cirri $27-38$ (28-34 in $89.5 \%)$; supraorbital cirri $4-14$ (6-11 in 
89.7\%); nasal cirri 4-19 (8-12 in $76.9 \%)$; LL tubes $1-11$ (2-9 in $89.4 \%)$; last LL tube positioned at point between verticals from dorsal-fin ray 7 and caudal-fin base ( 10 to caudal-fin base in $94.1 \%$ ); no scalelike flaps along LL; lower lip smooth mesially (plicate laterally); gill rakers 23-29 (based on 47 specimens); pseudobranchial filiaments on one side 7-10 (based on 47 specimens); premaxillary teeth 176-230 (based on 7 specimens); dentary teeth 83-96 (based on 7 specimens); upper 1 ip crenulae approximately 37-51; nuchal cirri in four groups (dorsalmost groups sometimes with cirri not connected at their bases) with bases of ventralmost groups slightly expanded basally, rarely with two groups on a side weakly connected basally (type B; Figure 7); first dorsal-fin spine of adults of both sexes 1-4 mm longer than second; dorsal-fin membrane deeply incised above last dorsal-fin spine; dorsal-fin membrane attached to caudal fin in adults; cephalic pore system complex ( 6 or more pores at most positions; Figure 17; number of pores increases with increasing SL); mid-snout pores present (Figure 17); extra interorbital pore position present (Figure 17); two pore positions behind nuchal flap (Figure 17); male genital papilla with urogenital orifice located basally between two widely separated slender filaments (less than $1.0 \mathrm{~mm}$ long) on a fleshy swelling behinci anus (type I; Figure 13); testes bulbous, length equals width; maximum SL about $85 \mathrm{~mm}$; ophioblennius larvae with 2 canines posteriorly on each dentary.

The smallest mature female (ova about 0.5 nm diameter) examined was about 55 SL, but a $35.6 \mathrm{~m}$ SL female from the Ogasawara Islands had developing ova (about 0.2 diameter). Males mature by about 35 m SL. 
Color in alcohol. Ophioblennius stage larvae (20-25 m SL) are uniformly pale brown, without blackish spots or other distinctive pigmentation.

Metamorphosed young (25-30 min SL) with melanophores uniformly distributed over body, but slightly darker than aphioblennius stage. Pale spots beginning to form on snout and under eye.

Subadults and adults of both sexes (Figure 62) with cream- to brown-colored head and body (some females with pupil-sized dark-brown spots on posterior half of body); anterior half of head covered with white spots (0.5-1.0 diameter), spots irregular in siape and frequently forming narrow vertical lines on snout and upper 1 ip; underside of head uniformly pale brown.

Color in life. Adults (based on color slides of freshly dead specimens from Enewetak Atoll, Abaiang, and Funafuti) with brown body (Funafuti specimens with body pinkish posteriorly and pale yellowish anteriorly); red spots and lines on head; nasal and supraorbital cirri reddish orange; nuchal cirri brown to black; pectoral-fin color ranging from pale brown to reddish; spinous-dorsal fin with reddish spines, membranes brown below anterodorsal translucent-triangular area and red streaks basally; segmented-dorsal fin with yellowish zays and red streaks extending from each ray base to mid-height of membrane between each pair of rays; upper caudal-fin rays yellowish, lower rays pale brown to yellowish; anal fin with reddish streaks basally, becoming brown in middle of fin, and tips of rays cream colored; iris color silver in the Funafuti and Abaiang specimens, red with yellow ring around pupil in the Enewetak specimen (iris color in the Enewetak 
specimen is tentative as the iris color had faded somewhat before the specimen was photographed).

A specimen collected at a depth of 30.5 m off Pitcairn Island differs from those described above in having the nasal cirri, supraorbital cirri, and dorsal half of head bright reddish orange; iris and other body colors same as described above for Enewetak specimen.

Comparisons. Of those Cirripectes species occurring on the Pacific plate, Cirripectes variolosus and $\underline{C}$. vanderbilti are the only ones with small white (red in life) spots and/or narrow lines on the snout and around the eye. Gosline (1955:469) stated that no "Hawaiian endemics" occurred sympatrically with their "Central Pacific counterparts" at Johnston Island. He failed to realize that his $\underline{C}$. variolosus comprised two species, $\underline{C}$. variolosus and $\underline{C}$. vanderbilti, which occur sympatrically at Johnston Island. They can be identified based on their nuchal cirri arrangement and the shape of the male genital papilla. Cirripectes variolosus has nuchal cirri type $B$ and male genital papilia type $I$, whereas $\underline{C}$. vanderbilti has types $G$ and $I I$, respectively (Figures 7 and 13 ).

Distribution. Cirripectes variolosus is endemic to the Pacific plate (sensu Springer, 1982) where it is known from every major island group except the Hawaiian Islands (Figure 26). It occurs as far west as the Ogasawara, Mariana, and Caroline Islands, in the northernmost Tonga Isiands, and as far east as the Pitcairn Island group. Although this species usually occurs on shallow reefs in water less than 5 m depth, it has been taken at a depth of 30.5 m at Ducie and Pitcairn Is lands. 
There is one collection of Cirripectes variolosus (ISNM 227978) that is supposedly from Aldabra Atoll, Indian Ocean. As this is the only record of this species from an area other than the Pacific plate, I believe the locality data for this lot are in error (see $\underline{C}$. jenningsi account for further discussion). I do not consider this record as part of the geographic range of $\underline{C}$. variolosus.

Etymology. The specific epithet is derived from the late Latin variola, meaning smallpox or spotted, and refers to the pattern of spots on the head.

Comments. Sexual dichromatism was :oted for some female specimens from the Marshall Islands, Johnston Island, Phoenix Islands, and Makatea. Some females have pupil-sized dark-brown spots on about the posterior half of body (Figure 63), while other females in the same populations have the unspotted bodies typical of both sexes in this species. Neither of these patterns is size dependent, as both patterns are present on young and adult females in the same collection. The reason for the variability in female color pattern (dark-brown spots on body or spots lacking) is unknown.

The specimens collected between 23 and $30.5 \mathrm{~m}$ depth in the Pitcairn Island group are problematical. These specimens occur in deeper water than any other known Cirripectes species. Other than the distinctive reddish-orange head. the characteristics of these specimens conform to wy definition of $\underline{C}$. variolosus. It is probable that the reddish-orange coloration of these specimens is an artifact of their depth of occurrence. In the absence of any additional infarmation substantiating the color differences, I treat these specimens merely as color variants . 
Nomenclatural discussion. Seale (1901) dessribed Salariae nigripes based on 30 specimens from Guam (also the type locality of Cirripectes variolosus). Although I have not examined the primary type material (BPBM 271), I have examined 15 paratypes (ANSP 82128), and all are

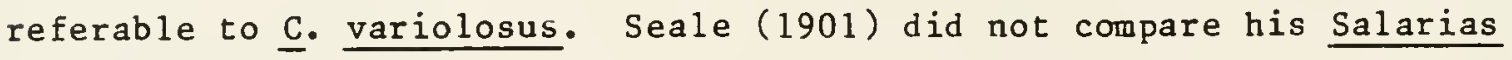
nigripes with any other species, but his description of the spots on the snout clearly associate his specimens with $\underline{\mathrm{C}}$. variolosus.

The type material used by Fowler (1938) in the description of Ophioblennius vanderbilti comprises two species. The paratypes from Christmas Island (ANSP 68434 to ó8445 inclusive) are ophioblennius stage larvae of Cirripectes variolosus. The specimens from Oahu are the types of $\underline{C}$ : vanderbilti.

The specimen referred to by Clark (1938) as Ophioblennius sp. indet. was used by Reid (1943) as the type of his $\underline{0}$ clarki. This specimen from Nukuhiva Island is an ophioblennius stage larva of Cirripectes variolosus.

Material examined. Types: MNHN 4411 (male: 38 m $\min$ SL), holotype of Salarias variolosus, Guam, Mariana Islands; CAS 24686 ( $1: 24$ ), holotype of Ophioblennius clarki, Marquesas Islands, Nuku Hiva Island, Taiohae Bay; ANSP 82128 (15: 25-46), paratypes of Salarias nigripes, Mariana Islands, Guam, Agana; ANSP 68434-46 (12: 19-21), paratypes of Ophioblennils vanderbilti, Christmas Island, Line Islands.

Other material examined. Ogasawara Islands (Bonin Islands): FAKU $48143(1: 36)$, FAKU $48144(1: 51)$, FAKU $48183(2: 42,48)$. Marcus Is land: BPBM 7157 (15), USNM 227949 (5: 36-61), USNM 227954 (7: 42-62). Wake Island: ANSP 65628-30 (3: 30-50), ANSP 65789-92 (4: 
28-30), ANSP $65802(2: 29,29)$, ВPBM 4925 (22), BPBM 15369 (2), CAS 38807 (22). Johnston Island: USNM 198731 (32: 32-67). Caroline Islands: BPBM UNCAT. ( $1 ; 10$ JUNE 1968), CAS UNCAT. ( $1 ;$ GVF 936), CAS $29246(6: 45-63)$, CAS 38805 (1: 47), CAS 38880 (2), CAS 48895 (1), CAS 48896 (2: 39, 51), CAS 48899 (1: 24), CAS 48917(3), CAS 48926 (1), CAS 48939 (8), CAS 48962 (2), CAS 48964 (1: 25), USNM 227633 (6:39-50). Mariana Islands: ANSP 82166 (1: 29), CAS 38798 (5), CAS 38879 (1), UF 31446 (3), UF 31447 (2). Marshall Islands: BPBM UNCAT. (1; 5 JULY 1975), BPBM 8249 (1), ВPBM 15520 (5), LACM 3979 (4), USNM 142107 (17: 35-66), USNM $142108(15: 30-75)$, USNM 142110 (7:47-59), USNM 142112 (11: 36-83), USNM $142113(26: 18-81)$, USNM 200614 (57 of $58: 31-62)$, USNM 227947 ( $1: 52$ ), USNM 228109 (2). Gilbert Islands: AMS I.18047-016 (2), BPBM 15328 (1), USNM 167333 (1 of 2: 56). Phoenix Islands: USNM 115493 (5: 34-38), USNM 115494 (48 of 49: 24-62), USNM 115496 (54 of 57: 23-62), USNM 227769 (1: 26), USNM 227951 (2:26, 26), USNM $227953(3: 46-78)$, USNM $227956(7: 26-53)$, USNM 227974 (2: 25, 26), USMM $227976(2: 28,44)$, USNM $227977(10: 25-48)$. Tokelau Islands: USNM $115495(3: 49-52)$, USNM $227948(1: 31)$. Samoa Islands: UF 31451 (1), USNM 115491 (4: 21-26). Cook Islands: BPBM 5741 (11), USNM 227955 (4: 26-43), USNM $227969(20: 23-51)$. Tubuai Islands: AMNH UNCAT. ( $1: 25)$. Tonga Islands: USNM 227952 (7:27-89). Society Islands: BPBM 8633 (1), BРBM 8642 (1), CAS 48963 (3), CAS 48974 (1), USNM 227960 (2: 25-38), USNM 227961 (2:38-45), USNM 227962 (1: 31 ), USNM 227971 ( $1: 51$ ), USNM 227972 (1: 38). Line Islands: ANSP 82159 (1: 39), ВРВМ $14060(1: 52)$, ВРВМ 4919 (2), ВРВМ 4920 (1), ВРВМ 7557 (9), BPBM 15156 (25), CAS 48949 (1), USNM 195809 (1), USNM 227950（1: 
40), USNM 227959 (3: 39-62), USMM 227970 (6: 36-42), USNM 227975 (29: 23-57). Tuamotu Archipelago: BPBM UnCAT. (5), BPBM UNCAT. (8: 47-62; 15 MARCH 1956), BPBM 12270 (4: 28-61), BPBM 16928 (2: 42, 43), BPBM 16941 (2: 25, 53), ВРВМ 17009 (1), ВРBM 17079 (4), CAS 48955 (9), CAS 48965 ( 1 : 84), USNM 227973 (1: 45). Marquesas Islands: AMS UNCAT. (1; OUT OF AMS I.22017-016), AMS I.21769-025 (3), BPBM UNCAT. (1:55; 11 OCT 1951), BPBM UNCAT. ( $1: 68 ; 10$ JULY 1957), BPBM UNCAT. (2: 49-68), ВРBM 11675 (12), ВPBM 11839 (2), BРBM 12113 (3), ВРBM 12143 (5), ВРВM 12803 (3).

As discussei earlier, the following lot appears to have incorrect locality data: USNM 227978 (7: 27-59), Aldabra Atoll. 


\section{Scartichthys JORDAN AND EVERMANN}

Scartes Jordan and Evermann, 1896:471 (type species: Salarias

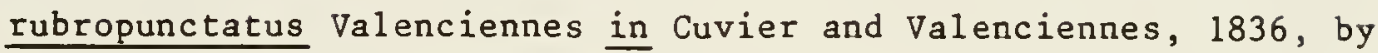
original designation; a junior homonym of Scartes Swainson, 1835, Mammalia).

Scartichthys Jordan and Evermann, 1898:2395 (type species: Salarias

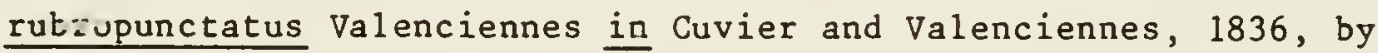
original designation).

Diagnosis. Scartichthys are salariin blennies with the posterior section of the lateral line comprising 18-21 short bi-pored tubes (continuous and tube sections of lateral line not in disconnected overlapping portions). Specimens of Scartichthys, with adults often exceeding $180 \mathrm{~mm}$ SL (up to $222 \mathrm{~mm}$ SL) are the largest (referring to a combination of length and weight) in the Blenniidae (the nemophin genus Xiphasia reaches $550 \mathrm{~mm}$ SL, but has an extremely slender body). Selected counts for the species of Scartichthys are provided in Table 4.

Distribution. The genus Scartichthys is restricted to the eastern Pacific Ocean, where it occurs from Panama to Chile (Figure 64). 
Figure 64. Geographic distribution of Scartichthys. S. (closed triangles), Scartichthys gigas (closed squares), s. variolatus (closed diamonds), and s. viridis (closed circles). 


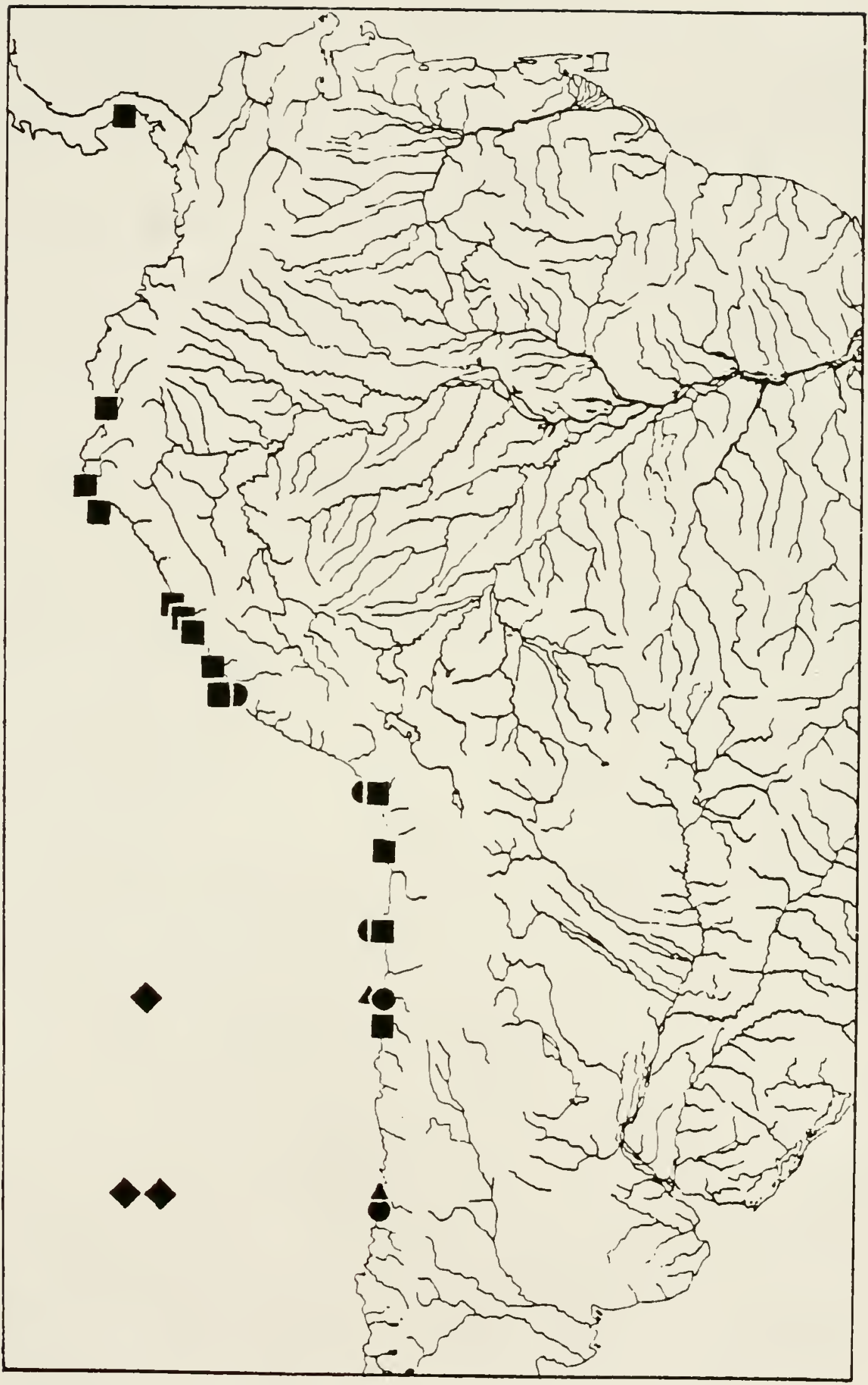


Table 4. Counts for selected characters of the species of Scartichthys. Table headings: 1st - primary modal count, 2nd apcondary modal count for bimodal characters, $\mathfrak{n}$ - number of specimens.

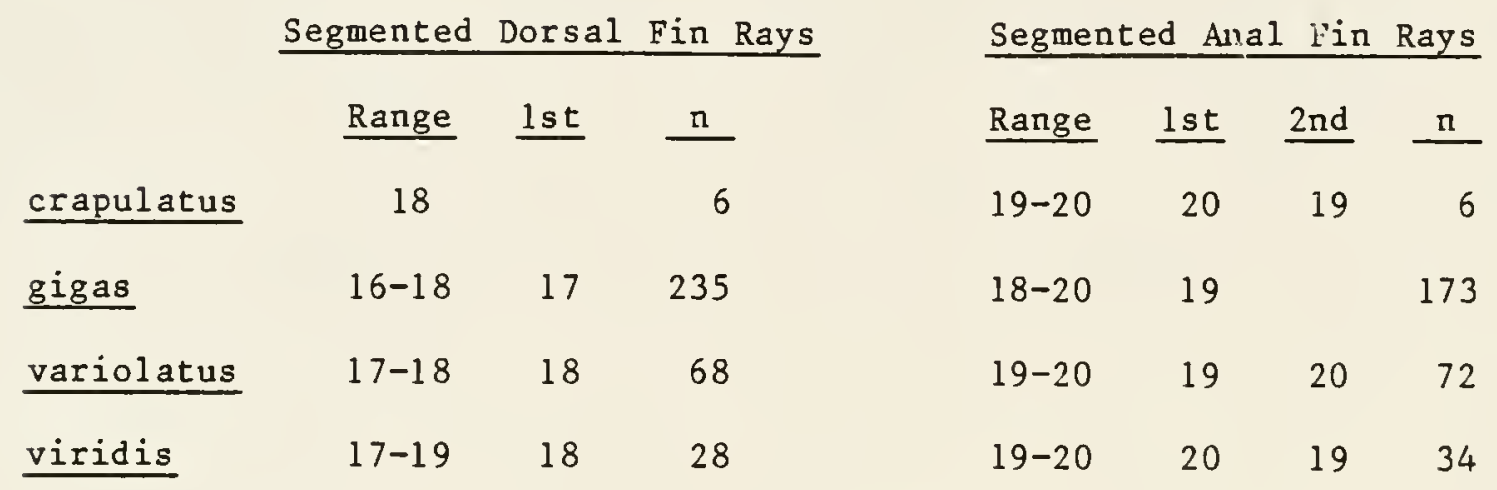

Vertebral Centrum Bearing

Last Pleural Rib

Range lst $\mathrm{n}$

crapulatus

$\underline{\text { gigas }}$

$\underline{\text { variolatus }}$

viridis
12

$11-12$

11

164

68

11

$11-12$

12

23
Caudal Vertebrae

Range 1st n

24

6

23-25 $24 \quad 166$

23-25 $24 \quad 69$

$23-25 \quad 24$

23 
Table 4. Continued.

Vertbral Centrum Bearing

Last Epipleural Rib

Range 1st 2nd $\mathrm{n}$

$\begin{array}{llll}\text { crapulatus } & 15-19 & 19 & 17\end{array}$

gigas

$\underline{\text { variolatus }}$

viridis

$15-20 \quad 17 \quad 18 \quad 156$

Left+Right Orbital Cirri

Range 1st 2nd n

$18-28 \quad 22$

6

$\begin{array}{llll}19-53 & 29 & 39 & 15\end{array}$

$22-116$

23

$20-51 \quad 30$

16 $\underline{\text { Left+Right Nasal Cirri Total Nuchal Cirri Bases }}$ Range 1st 2nd $\underline{n} \quad \underline{\text { Range }} \underline{1 s t} \underline{n}$

crapulatus

$7-9 \quad 8$

4

$7-20 \quad 8$

6

$\underline{\text { gigas }}$

$7-15 \quad 8$

23

8-19 12

16

$8-31 \quad 13$

30

6-15 9

8

18
21

$6-60 \quad 22 \quad 16$ 
Table 4. Continued.

Dentary Teeth

Range lst 2nd $n$

$\begin{array}{llll}\text { crapulatus } & 92-110 & 110 & 6\end{array}$

$\begin{array}{llll}\text { gigas } & 57-69 & 60 & 30\end{array}$

$\begin{array}{lllll}\text { variolatus } & 74-93 & 87 & 88 & 31\end{array}$

$\begin{array}{lll}\text { viridis } & 106-131 & 120\end{array}$ 
Key to the Species of Scartichthys

1a. Fewer than 94 dentary incisors; body coloration mottled with brown

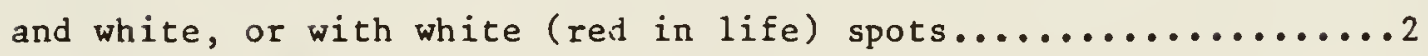

1b. More than 94 dentary incisors; body uniformly brown, occasionally with a pupil-sized dark-brown midlateral stripe, or with tiny

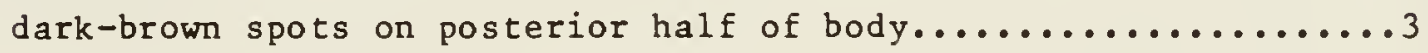

2a. Dentary incisors 73-94; typically 18 segmented dorsal-fin rays; pupil-sized black spot on first (sometimes on second also) interspinal membrane of dorsal fin; pupil-sized white spots over head and body; occurs on offshore islands of Chile.....variolatus

2b. Dentary incisors 57-69; typically 17 segmented dorsal-fin rays; no pupil-sized black spot on first interspinal membrane of dorsal fin; body mottled with brown and white; occurs from Panama to

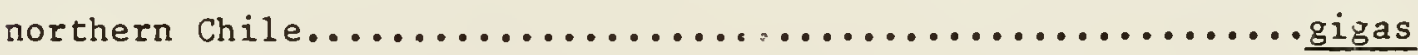

3a. Tiny dark-brown spots on posterior half of body; known only from northern Chile and from the vicinity of Valparaiso.......crapulatus

3b. Body coloration uniformly brown, occasionally with a dark-brown stripe (its width about equal to diameter of pupil); occurs from vicinity of Valparaiso, Chile, to southern Peru.......... viridis 
Figure 65. Female Scartichthys crapulatus, holotype, Chile (USNM 276344: $110 \mathrm{~mm} \mathrm{SL).}$

Figure 66. Female Scartichthys gigas, Peru (GCRL 12635: $140 \mathrm{~mm}$ 

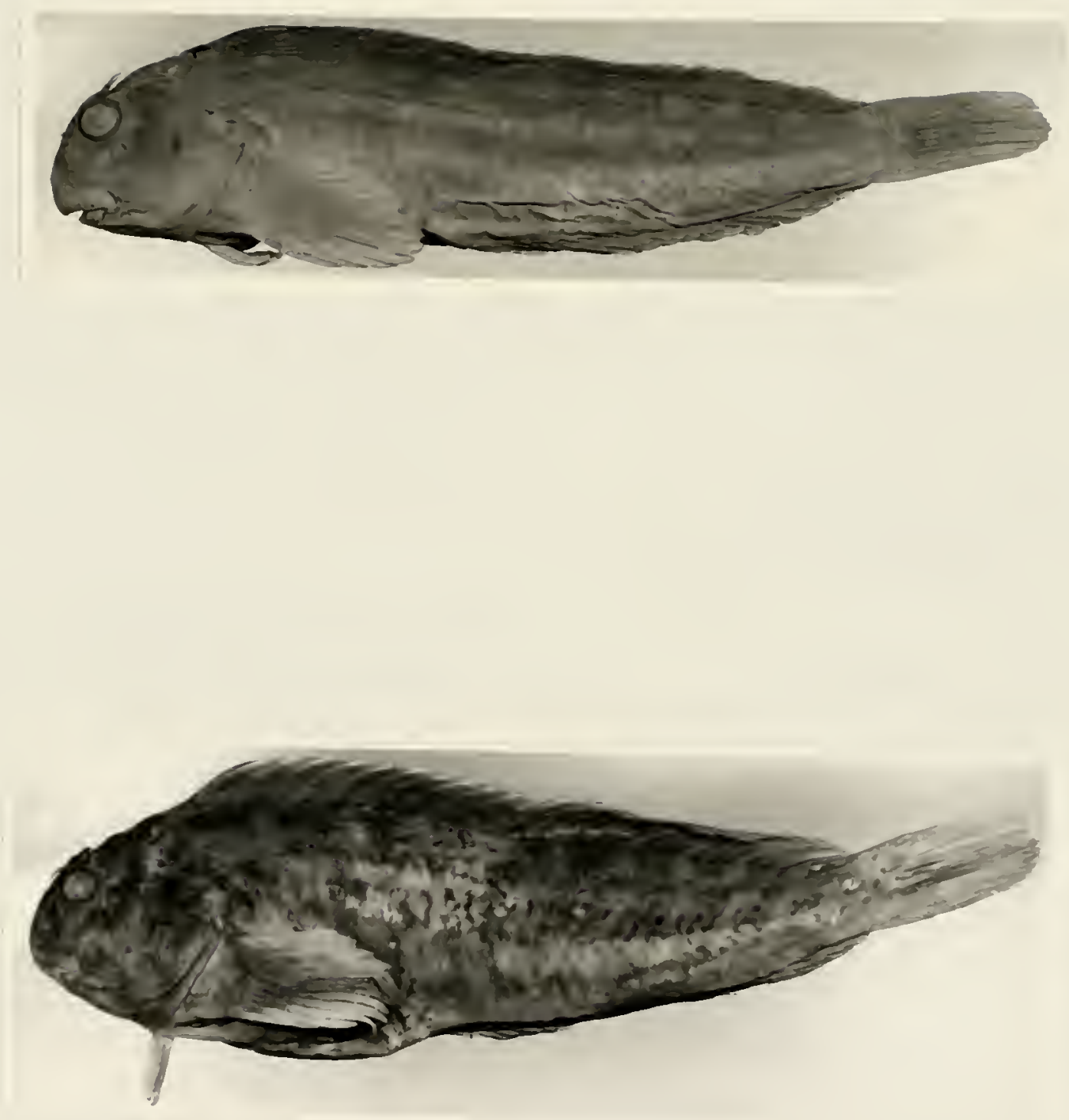
Figure 67. Female Scartichthys variolatus, Juan Fernandez Islands (MNHNC P.6.227: $103 \mathrm{~mm}$ SL). SL ).

Figure 68. Female Scartichthys viridis, Chile (GCRL 12618: $121 \mathrm{~mm}$ 

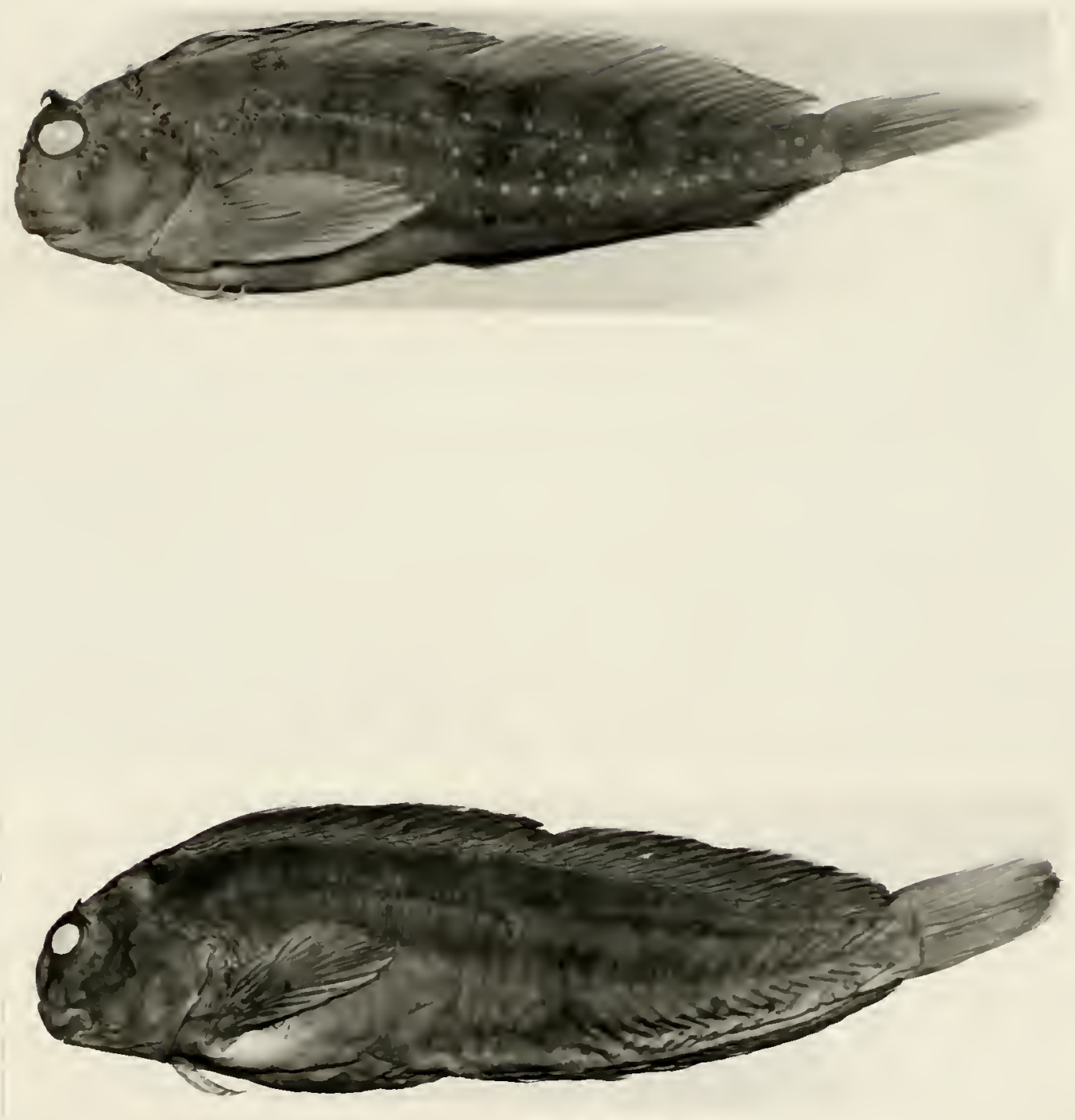
Scartichthys crapulatus new species

Diagnosis. A species of Ecartichthys with more than 95 dentary incisors and small dark-brown spots on posterior half of body.

Description. Dorsal fin XII, 18; anal fin II, 19-20 (20 in 6 of 9 specimens); total procurrent caudal-fin rays $14-15$; pelvic fin $I, 4$; vertebrae $10+24=34$; 1 ast pleural ribs on vertebral centrum 12 ; last epipleural rib on vertebral centrum $15-19$ (17-19 in 5 of 6$)$; anal pterygiophores $2-1-1$ or $1-2-1$ (1-2-1 in 5 of 6$)$; nuchal cirri 7-2ú (6-12 in 5 of 6); supraorbital cirri 18-28; nasal cirri 7-9; LL tubes 21-22 (21 in 8 of 10); last LL tube at base of caudal fin; no scalelike flaps along LL; lower lip smooth mesially (plicate laterally); gill rakers 22-27 (based on 5 specimens); pseudobranchial filaments on one side 10-11 (based on 6 specimens); premaxillary teeth 205-220 (based on 3 specimens); dentary teeth $78-110$ (95-110 in 10 of 14 ; fewer than 95 only in specimens less than $50 \mathrm{~mm}$ SL); upper lip crenulae approximately 28-36; nuchal cirri in 2 groups widely separated across nape (Figure 7); first dorsal-fin spine of adults approximately equal in length to second in both sexes; dorsal-fin membrane deeply incised above last dorsal-fin spine; dorsal-fin memurane attached to caudal peduncle in advance of caudal fin in adults; cephalic pore system relatively simple (less than 3 pores at most positions; number of pores increases with increasing SL); male genital papilla with urogenital orifice located basally between two small protuberances on a fleshy swelling behind 
anus (Figure 13); testes elongate, length equals about twice width; maximum SL about $110 \mathrm{~mm}$.

No mature females of this species have been examined, but the female holotype (about $110 \mathrm{~mm}$ SL) has well-developed ovaries and females less than $70 \mathrm{~mm}$ SL do not. No mature males have been examined. Color in alcohol. Juveniles have brown pupil-sized stripe midlaterally on body on pale background, nine to eleven saddlelike blotches along dorsal profile, posterior half of body speckled with tiny black spots; head brown with small black spot, its diameter equal to about half diameter of pupil, behind posterior margin or eye; anterior one to three interspinal membranes of dorsal fin with black pupil-sized spot, remainder of fins dusky.

Adult female (Figure 65) with background color of head and body brown, faint eye-sized dark-brown stripe midlaterally on body, posterior half of body with small dark-brown spots; dorsal and anal fins with narrow pale distal margins.

Color in life. Life rolors not known.

Comparisons. Scartichthys crapulatus is the only member of the genus with small dark-brown spots on the posterior half of the body. It is most similar in meristics and other counts to $\underline{s}$. viridis, but is easily distinguished by color pattern (the latter has a uniformly brown body).

Distribution. Scartichthys crapulatus is known only from the coast in the vicinity of Valparaiso, Chile, and from a power plant trap in the northern Chilean town of Barquito, where it was collected with

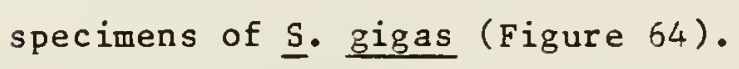


Etymology. The specific epithet, the Latin crapulatus, meaning drunk, refers to the purported drunken feeling experienced when the flesh of Scartichthys is eaten.

Material examined. Holotype: USM 276344 (female: 110 m SL), Montemar Biological Station, field number BBC 1155 , collected by B. B. Collette and R. H. Gibbs, Jr., 14 Feb. 1966.

Paratypes: ANSP UNCAT. (4 specimens: 42-64 mim SL), Chile, Barquito, power plant trap, 28 April 1955; BMNH 1850.6.14:20 (1: 67), South America (probably Chile), collected by Mr. D̈ridges; MNHNC P.6.411 (8: 37-78), Chile, El Quisco, $32^{\circ} 23^{\prime} \mathrm{s}, 71^{\circ} 42^{\prime}, 14$ Sept. 1952.

\section{Scartichthys gigas (Steindachner)}

Salarias gigas Steindachner, 1876:220 (Callao, Peru; syntypes NMW $73438: 1$ and $73438: 2)$.

Salarias eques Steindachner, 1898:307 ("Cavanchabai bei Iquique"; holotype ZMB 15658).

Ophioblennius xiphiodon Clark, 1938:183 (Callao, Peru; holotype CAS $5543)$.

Ophioblennius mazorkae Hildebrand, 1946:384 (Mazorka Island, Huaura Group, Peru; holotype 128188).

Diagnosis. A species of Scartichthys with fewer than 74 dentary incicors and a reticulated color pattern.

Description. Dorsal fin XI-XIII (XII in $96.6 \%$ of specimens), 16-18 ( 17 in $91.9 \%$ ); anal fin II, 18-20 (19 in $80.9 \%$; $18-19$ in $99.4 \%$ ); total 
procurrent caudal-fin rays 10-16; pelvic fin 1,4 ; vertebrae $10-11$ (10 in $99.4 \%)+23-25(24$ in $94.6 \%)=33-35(34$ in $94.6 \%)$; last pleural rios ou vertebral centrum 11-12 (11 in $99.4 \%$ ); last epipleural rib on vertebral centrum 15-20 (17-19 in 92.9\%); anal pterygiophores 2-1-1, 1-2-1, or 1-1-2 (1-2-1 in 75.6\%); nuchal cirri 8-19; supraorbital cirri 19-53 (23-39 in $80.0 \%)$; nasal cirri 7-15 (7-11 in 95.7\%); LL tubes 18-22 (19-21 in 87.5\%); last LL tube on caudal-fin base; no scalelike flaps along LL; lower lip smooth mesially (plicate laterally); gill rakers 16-20 (based on 10 specimens); pseudobranchial filaments on one side 9-13 (based on 12 specimens); premayi1lary teeth 108-146 (based on 10 specimens); dentary teeth $57-69$ (based on 30 specimens); upper 1 ip crenulae approximately 24-31; nuchal cirri in two irregular groups on either side of the nape, distance between them approximately equal to diameter of eye; length of first dorsal-fin spine of adults about equal to second in both sexes; dorsal-fin membrane deeply incised above last dorsal-fin spine; dorsal-fin membrane attached to caudal peduncle in advance of caudal fin in adults; cephalic pore system complex ( 4 or more pores at most positions; number of pores increases with increasing SL); mid-snout pores present (Figure 17); male genital papilla with urogenitai orifice located basally between two small protuberances on a fleshy swelling behind anus (Figure 13); maximum SL about $222 \mathrm{~mm}$; ophioblennius larvae with $2-3$ canines posteriorly on each dentary; no spets on membrane between anterior dorsal-fin spines.

The smallest mature female (ova about 0.6 mm diameter) examined was about $126 \mathrm{~mm} \mathrm{SL}$, but it is likely that in some populations, females mature at somewhat smaller sizes. Males mature by about $90 \mathrm{~mm}$ SL. 
Color in alcohol. Ophioblennius stage larvae dale with 11 to 13 saddlelike blotches of pigment along dorsal aspect of body; distal half of pectoral fin with numerous melanophores on rays and membranes; melanophores at tips of spinous dorsal-fin rays; remainder of fins pale; small pupil-sized cluster of melanophores behind midpoint of posterior margin of eye.

Juveniles with about eight to ten irregular dark-brown bars with pale interspaces along body; head irregularly covered with pale spots and bars, and dark-brown bars, large black spot behind posterior margin of orbit, size of spot about half to three-quarters size of eye; dorsal and anal fins dark brown with pale-tipped fin elements; other fins dusky.

Adult color pattern (Figure 66) similar to that of juveniles except the bars on the head and body break up into a reticulated pattern. Color in life. A recently collected adult male in alcohol still retained some orange color at the distal tips of the dorsal-fin Q ements. Other colors are unknown.

Comparisons. Scartichthys gigas differs from all other

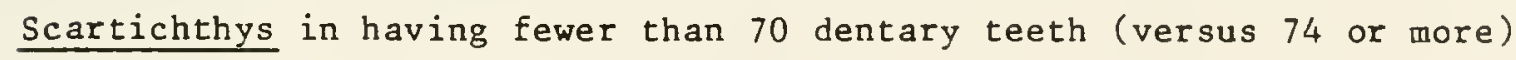
arid in having a vermiculated color pattern over head and body (all others have spots or a uniformly pigmented body).

Distribution. Scartichthys gigas occurs from the Pacific coast of Panama to Antofagasta, Chile (Figure 64). It is sympatric with $\underline{\mathrm{s}}$.

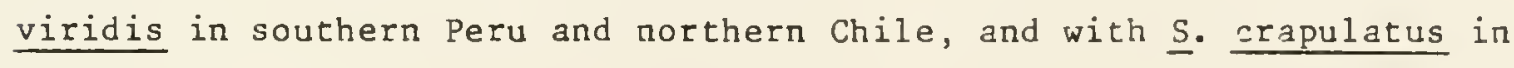
northern Chile.

Etymology. The specific epithet is Latin for giant and refers to the relatively large size of adults. 
Nomenclatural discussion. Steindachner (1876) described Salarias gigas based two specimens from Callao, Peru, but gave no catalog numbers. The two syntypes of $\underline{\mathrm{S}}$ gigas are cataloged as NMW 73438:1 (female) and 73438:2 (male), from Callao, Peru.

Salarias eques was described by Steindachner (1898) based on a single specimen from "Cavanchabai bei Iquique." Steindachner treated

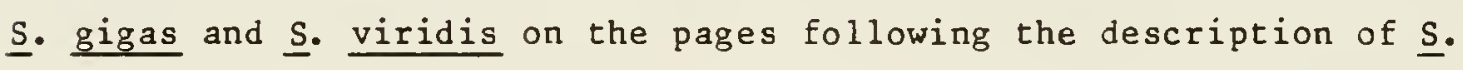

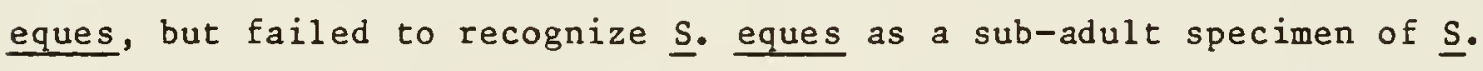
gigas. The holotype of $\underline{\mathrm{S}}$. eques is cataloged as ZMB 15658 (female, 86 mn SL). Although the holctype is a sub-adult, it retains the striped pigmentation pattern frequently found on much smaller specimens. The low number of dentary teeth and the lack of a spot anteriorly in the dorsal fin are characteristic of Scartichthys gigas. Salarias eques is a junior synonym of $\underline{\text { S. gigas. }}$.

Clark (1938) described Ophioblennius xiphiodon based on ophioblennius stage post-larval specimens from Peru and Chile. As I discuss in the account of Scartichthys viridis, the paratypes from

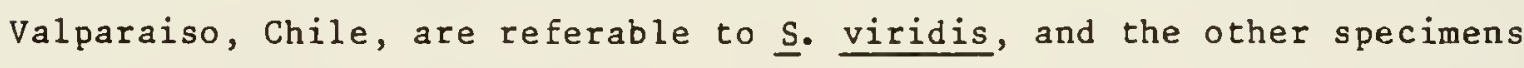
probably belong to $\underline{\mathrm{s}}$. gigas. The Peruvian holotype was not available for examination, but, as the Peruvian paratypes are post-larvae of $\underline{s}$. gigas and the collection locality (Callao) of the holotype is the same as that of the types of $\underline{S}$. gigas, I consider Ophioblennius xiphiodon to be a junior synonym of $\underline{s}$ gigas.

Ophioblennius mazorkae was described by Hildebrand (1946) based on ophioblennius-stage post-larval specimens of Scartichthys gigas and, thus, is a junior synonym of the latter. 
Material examined. Types: NMW 73438:1 (female: 150 SL) and 73438:2 (male: 185), syntypes of Salarias gigas, Callao, Peru; ZMB 15658 (1: 84), holotype of Salarias eques, Iquiqui, Chile; CAS 5544-5550 ( 3 of 7 examined: 33-41), paratypes of Ophioblennius xiphiodon, Peru, Callao; CAS 5553-5556 (2 of 4 examined: 31, 32), Peru, Chinchas; USNM 128188 (1: 33), holotype of Ophioblennius mazorkae, Peru, Huauru Group, Mazorka Island; USNM 128189 (20 of 60+ examined: 30-36), paratypes of Ophioblennius mazorkae, Peru, Huauru Group, Mazorka Island.

Other material examined. West Coast of South America (exact locality unknown): BMNH 1908.12.5.32-3 (2: 54, 57). Panama: USNM 144797 (72: 30-58). Ecuador: USNM 88796 (3: 106-120). Peru: GCRL 12492 (1), GCRL $12513(3: 127-159)$, GCRL 12628 (9), GCRL $12633^{\circ}$ (6), GCRL 12635 (25), NMW 20516 (1), NMW 22184 (1), NMW 73244 (2: 102, 103), NMW 73425 (2: 117-141), RMNH 11283 (1:193), RMNH 11462 (1: 167), SIO 65-602 (12), UF $21437(1: 136)$, USNM UNCAT. (2: 135, 135; GCRL 1670); USNM UNCAT. (4: 37-55; 6 JUNE 1966); USNM UNCAT. (5: 120-153; GCRL 1677); US NM UNCAT. (4: 33-57; GCRL 1672); USNM UNCAT. (1:63; LWK 66-84), USNM $102009(1: 50)$, USNM $102010(1: 65)$, US NM $128192(2: 30,52)$, US NM 128195 ( $1: 43$ ), USNM 200396 (1: 45), USNM 227556 (57 of 100+ examnined: 47-108), USNM 227981 (100+), ZMB 9058 (1:220), ZMB 9107 (1), ZMB 13740 (4), ZMB 13741 (6). Chile (exact locality unknown): BMNH 1871.4.13.26-7 (2: 138-222). Chile: USNM $76389(1: 71)$, USNM 122571 (7: 40-126), USNM 175368 (1: 78), ZMB 15659 (1), ZMB 15660 (3), ZMB 15662 (5). 
Scartichthys variolatus (Valenciennes)

Salarias variolatus Valenciennes in Cuvier and Valenciennes, 1836:346

(Juan Fernandez Island; holotype MNHN 4410).

Salarias rubropunctatus Valenciennes in Cuvier and Valenciennes,

1836:346 (Juan Fernandez Island; syntypes MNHN A.2038).

Ophioblennius fernandezensis Clark, 1938:184 (San Juan Bautista

[Cumberland] Bay, Juan Fernandez Islands).

Diagnosis. A species of Scartichthys with the following combination of characters: $73-94$ dentary incisors, 18 segmented dorsal-fin rays, pupil-sized black spot on first and somtimes second interspinal membrane, and pupil-sized white spots on head and body.

Description. Dorsal fin XI-XII (XII in $98.6 \%$ of specimens), 17-18 ( 18 in $91.2 \%$ ); anal fin II, 19-20 (19 in 56.9\%); total procurrent caudal-fin rays $12-14$ ( 1 specimen with 16); pelvic fin I,4; vertebrae $10+23-25(24$ in $94.2 \%)=33-35(34$ in $94.2 \%) ;$ last pleural ribs on vertebral centrum 11; last epipleural rib on vertebral centrum 16-19 (17-18 in $91.2 \%)$; anal pterygiophores $2-1-1,1-2-1$, or $1-1-2(1-1-2$ in 76.8\%); nuchal cirri 20-95 (20-77 in 95.2\%); supraorbital cirri 22-116 (19-74 in $91.7 \%)$; nasal cirri $8-31$ (10-23 in $90.0 \%)$; LL tubes $19-21$ (20-21 in $90.0 \%)$; last LL tube on base of caudal fin; no scalelike flaps along LL; lower lip entire mesially (plicate laterally); gill rakers 21-28 (based on 16 specimens); pseudobranchial filaments on one side 10-15 (based on 16 specimens); premaxillary teeth 200-223 (based on 2 specimens); dentary teeth $80-93$ (based on 30 specimens); upper 1 ip 
crenulae approximately 35-43; nuchal cirri in 2 widely separated transverse rows on either side of nape; first dorsal-fin spine of adults approximately equal to second in males and females; dorsal-fin membrane deeply incised above last dorsal-fin spine; dorsal-fin membrane attached to caudal peduncle in advance of caudal fin in adults; cephalic pore system complex ( 3 or more pores at most positions; number of pores increases with increasing SL); mid-snout pores present (Figure 17); male genital papilla with urogenital orifice locsted basally between two short protuberances on a fleshy swelling behind anus (Figure 13); testes elongate, length more than twice width; maximum SL about $150 \mathrm{~mm}$; pupil-sized black spot on distal portion of membrane between first and second dorsal-fin spines $\left(93^{\circ} .5 \%\right.$ of specimens) and occasionally a second spot on membrane between second and third spines $(6.5 \%)$.

The smallest mature female (ova about 0.8 mm diameter) examined was about 100 man SL. Males mature by about 100 SL.

Color in alcohol. Subadults and adults of both sexes (Figure 67) have a dark brown background coloration with pupil-sized pale spots over head and body, and 7 to 9 broad, faint, dark-brown bars on body; dorsal fin with pupil-sized black spot on first interspinal membrane, remainder of fin dusky with small pale spots, dorsal and anal fins with distal ends of elements pale tipped; caudal fin brown with small pale spots; black spot about half diameter of pupil on head behind posterior margin of eye.

Color in life. I have not seen the life colors of this species, but Valenciennes in Cuvier and Valenciennes (1836) describes the spots on 
the body as brownish red. An illustration in the Cuvier and Valenciennes manuscript material in the library of the Museum National d'Histoire Naturelle, Paris, clearly shows the brownish-red spots described in their 1836 publication. Other colors are the same as described for specimens in alcohol.

Comparisons. Scartichthys variolatus differs from other Scartichthys in that adults have 74-93 dentary teeth. Scartichthys

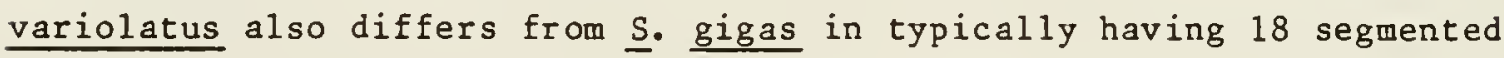
dorsal-fin rays (versus 17 modally). The distinctive color pattern of Scartichthys variolatus comprising pale (brownish-red in life) spots on a dark background will distinguish this form from all other Scartichthys.

Distribution. Scartichthys variolatus is an island endemic known only from San Felix and San Ambrosio islands, and Juan Fernandez Islands (Figure 64).

Etymology. The specific epithet is derived from the late Latin variola, meaning smallpox or spotted, and refers to the pattern of spots on the head and body.

Nomenclatural discussion. Valenciennes in Cuvier and Valenciennes (1836) described specimens belonging to Scartichthys variolatus under two species names, Salarias variolatus and $\underline{S}$. Iubropunctatus. The former description was based on an adult specimen and the latter on five young specimens from the same island. The syntypic series of $\underline{S}$. rubropunctatus comprises four specimens belonging to Scartichthys variolatus, and one specimen with supraorbital and nuchal cirri morphologies similar to $\underline{\mathrm{S}}$ viridis and number of dentary teeth in the 
range for $\underline{s}$. variolatus. As no other specimens of $\underline{s}$. viridis have been collected from the Juan Fernandez Islands, I consider the last specimen

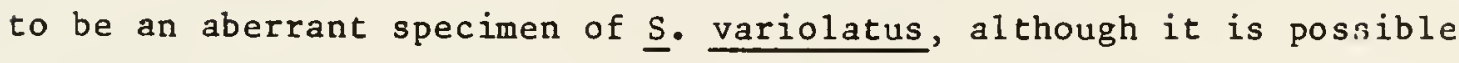
that Valenciennes inadvertently mixed some of his mainland Chile and Juan Fernandez Islands collections. To avoid future confusion, I designate a 43 mim SL male (MNHN A.2038) as lectotype of Salarias rubropunctatus. The name $\underline{S}$. variolatus is the senior synonym, based on page priority, and $\underline{S}$. rubropunctatus is a junior synonym.

Clark (1938) described Ophioblennius fernandezensis based on eight specimens from the Juan Fernandez Islands. I have not examined the holotype (CAS 5557), but my examination of 3 of the 7 paratypes, the original description, and data taken from the holotype by V. G. Springer clearly indicate that this species was described based on ophioblennius larval stage specimens of Scartichthys variolatus. Thus, Ophioblennius fernandezensis is a junior synonym of Scartichthys variolatus.

Material examined. Types: MNHN 4410 ( 1 specimen: 133 mm SL), holotype of Salarias variolatus, Ile Juan Fernandez; MNHN A.2038 (1: 43), lectotype of Salarias rubropunctatus, Ile Juan Fernandez; MNHN uncat. (4: 43-59), paralectotypes of Salarias rubropunctatus, Ile Iuan Fernandez; CAS 5558 through 5564 (examined 3 of 7 specimens: 40-42), paraytpes of Ophioblennius fernandezensis, Juan Bautista Bay, Juan Fernandez Island.

Other material examined. Juan Fernandez Islands: BMNH 1935.9.10.19 (1: 117), NMW 73428 (1), MNHNC P.6.073 (4), MNHNC P.6.212 (1: 163), MNHNC P.6.230 (1), MNHNC P.6.408 (1), SIO 65-638 (41), USNM 
176540 ( $1: 60)$, USNM 176546 (11:45-97), USNM [ (1:67), ZMB 15661 (3), ZMB 15663 (4). Isla San Felix: MNHNC P.6.406 (1), SIO 65-624（65: 42-148). San Ambrosio Island: MNHNC P.6.166 (2), MNHNC P.227 (20).

\section{Scartichthys viridis (Valenciennes)}

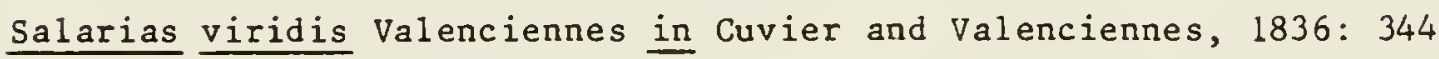
(Valparaiso; holotype MNHN A-2128).

Salarias cuvieri Gunther, 1861:248 (Chile; syntypes BMNH 1850.6.14.19 and $1850 \cdot 6 \cdot 14 \cdot 21)$.

Blennophis semifasciatus Kner and Steindachner, 1866:369 (near Iquique, west coast of South America).

Salarias concolor Philippi, 1896:380 (Valparaiso; type specimen not located).

Salarias modestus Philippi, 1896:381 (not specified but presumably in Chile; type specimen not located).

Salarias petersoni Fowler, 1940:189 (Antofagasta, Chile; holotype ANSP $69150)$.

Diagnosis. A species of Scartichthys with more than 94 dentary incisors and a uniformly brown body, sometimes with a dark-brown lateral stripe.

Description. Dorsal fin XII, 17-19 (18 in $78.6 \%$ nf specimens); anal fin II, 19-20; total procurrent caudal-fin rays $13-16$; pelvic fin I,4; vertebrae $10+23-25(24$ in $82.6 \%)=33-35(34$ in $82.6 \%$ ); last pleural ribs on vertebral centrum 11-12 (12 in 69.6\%); last epipleural rib on 
vertebral centrum 16-21; anal pterygiophores $1-1-2,1-2-1$, or 2-1-1 (1-2-1 in $65.2 \%)$; nuchal cirri 6-60 (6-40 in $88.9 \%)$; supraorbital cirri 20-51 (20-32 in 87.5\%); nasal cirri 6-15 (6-12 in 88.9\%); LL tubes 20-22 (21 in $63.6 \%$ ); 1ast LL tube on base of caudal fin; no scalelike flaps along LL; lower 1 ip smooth mesially (plicate laterally); gill ]akers 20-26 (based on 13 specimens); pseudobranchial filaments on one side 9-14 (based on 14 specimens); premaxillary teeth 226-268 (based on 5 specimens); dentary teeth 106-131 (based on 26 specimens; 93 in a 47 mm SL specimen); upper 1 ip crenulae approximately $30-42$; nuchal cirri in 2-4 groups of irregularly shaped cirri with broad separation across nape (Figure 7 ); first dorsal-fin spine of adults approximately equal to second in both sexes; dorsal-fin membrane deeply incised above last dorsal-fin spine; dorsal-fin membrane attached to caudal peduncle in advance of caudal fin in adults; cephalic pore system relatively simple (less than 3 pores at most positions); mid-snout pores present (Figure 17); male genital papilla with urogenital orifice located basally between two small protuberances on a fleshy swelling behind anus (Figure 13); testes elongate, length more than cwice width; maximum SL about $200 \mathrm{~mm}$; pupil-sized black spot on distal portion of membrane between first and second dorsal-fin spines and usually a second spot on membrane between second and third spines.

The smailest mature female (ova about 0.8 mu diameter) examined was about 170 um SL, but females have fully developed ovaries by about 100 mm SL. Males mature by about 100 m SL.

Color in alcohol. Diffuse pupil-sized black spot behind middle of posterior margin of eye at all sizes (often difficult to discern in 
dark specimens). Ophioblennius stage larvae pale with 9 or 10 blotches on dorsum, pectoral fin with distal half black and proximal half pale. Adults of both sexes (Figure 68) uniforrly dark brown dorsally, becoming paler ventrally, and sometimes with midlateral dark-brown stripe (width about equal to eye diameter); all fins dusky; dorsal fin with pale area over distal tips of anterior three or four spines, other spines pale tipped, black pupil-sized spot on each of first two or three interspinal membranes, spots located ventral to pale area. Color in life. Valenciennes in Cuvier and Valenciennes (1836) describes the life colors as dark green on the back and somewhat paler green on the stomach.

Comparisons. Scartichthys viridis is distinctive in having a relatively uniformly pigmented body without spots. It is further differentiated from $\underline{S}$. gigas and $\underline{\text { S. variolatus }}$ in having a high number of dentary teeth, 106-131 (versus 93 or fewer).

Distribution. Scartichthys viridis occurs from Valparaiso, Chile northward to Independencia Bay, Peru (Figure 64). Specimens have been collected from tidepools and rocky shore areas.

Etymology. The specific epithet, viridis, is Latin for green, referring to the green color of the body is. life.

Nomenclatural discussion. Valenciennes in Cuvier and Valencienres (1836) described Salarias viridis based on a single specimen (MNHN A.2128) From Valparaiso, Chile.

The description of $\underline{S}$. cuvieri by Gunther (1861) is problematical in that it appears to be based on two species. He cited data in Valenciennes' description of $\underline{S}$. variolatus, which he placed in the 
synonymy of $\underline{\mathrm{S}}$. cuvieri, but based the description on two specimens (BMNH 1850.6.14.19 and 1850.6.14.21). The specimens he listed as material of $\underline{S}$. cuvieri are referable to scartichthys viridis. Smith-Vaniz and Springer (1971) listed these specimens as primary types of $\underline{S}$. cuvieri. As there are type specimens for $\underline{s}$. cuvieri, I consider the description to apply to these specimens. To avoid future confusion, I designate one of these (BMNH 1850.6.14.19, 196 mm SL male) as the lectotype of Salarias cuvieri, and consider it a junior synonym of $\underline{\text { s. viridis. }}$

Kner and Steindachner (1866) described Blennophis semifasciatus based on an ophioblennius stage larva. Although Smith-Vaniz and Springer (1971) correctly placed it in the genus Scartichthys, the characters given in the original description are not sufficient for assigning the specimen to a particular species of Scartichthys, but the figure of the type generally resembles ophioblennius larvae of Scartichthys viridis. I searched the collection of fishes in Vienna, Austria, where the type was originally deposited, but could not find the specimen. In addition, the type specimen was not found by $V . G$. Springer (pers. comm.) in the Hamburg Museum, where many other Goddefroy types are located. To av id future confusion, I have selected a neotype (USNM 194480, an ophioblennius larva of $\underline{\text { S. }}$ viridis from northern Chile) for Blennophis semifasciatus and consider

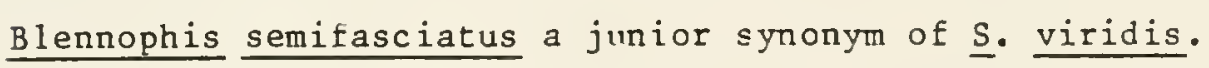

The type specimens of Salarias concolor and $\underline{s}$. modestus, described by Philippi (1896), could not be located (may be lost) but appear to be referable to Scartichthys viridis, based on Philippi's descriptions. 
Salarias concolor was described by Philippi (1896) from Algarrobo and Valparaiso. This distribution limits the number of species to which it can belong and, combined with Philippi's (1896) description, clearly

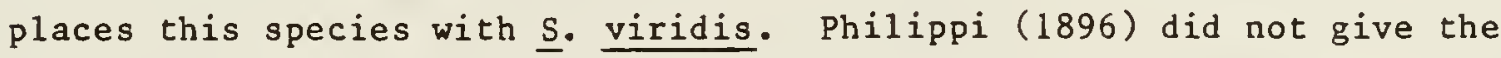
geographic distribution of $\underline{\mathrm{S}}$. modestus. He compared his specimens with

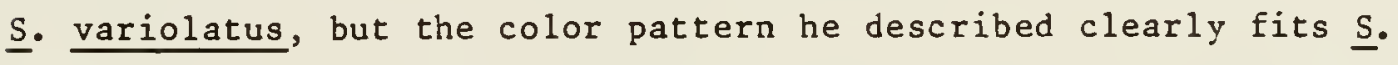
viridis. Thus, I place $\underline{\text { s. modestus }}$ in the synonymy of $\underline{\text { s. viridis. }}$ Clark (1938) described Ophioblennius xiphiodon based on a mixture of specimens belonging to Scartichthys gigas and $\underline{\text { S. viridis. Based on }}$ the collection locality of the holotype, I place $\underline{0}$. xiphiodnr in the synonymy of $\underline{\mathrm{S}}$ gigas. The two paratypes from Valparaiso, Chile, are

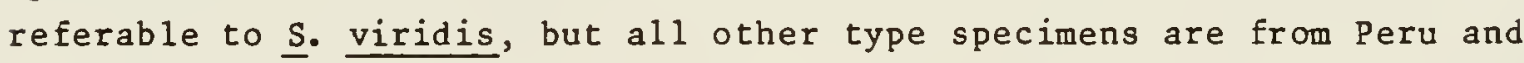
presumably belong to $\underline{\text { S. }}$ gigas.

Fowler (1940) described Salarias petersoni based on a metamorphosing specimen from Antofagasta, Chile, that is referable to Scartichthys viridis. The holotype (ANSP 69150) has lost the large canines typical of ophioblennius stage larvae, but retains the larval pigmentation pattern consisting of dark blotches along the dorsum. Material examined. Types: MNHN A.2128 ( 1 specimen: 197 m SL), holotype of Salarias viridis, Valparaiso; BMNH 1850.6.14.19 (1: 196), lectotype of Salarias cuvieri, South America; BMNH 1850.6.14.21 (1: 142), paralectutype of Salarias cuvieri, South America; USNM 194480 ( 1 : 45), neotype of Blenrophis semifasciatus, "Atlantis" Cruise $221,24^{\circ}$ $54^{\prime} \mathrm{S}, 71^{\circ} 26^{\prime} \mathrm{W}$, dip netted under a night light by S. Bray, 10 December 1955; CAS 5552 (1: 40), paratype of Ophioblennius xiphiodon. Vlaparaiso Harbor, Chile; ANSP 69150 (1: 46), holotype of Salarias petersoni, Antofagasta, Chile. 
Other material examined. Chile: NMW 73462 (1: 128), RMNH 1810 (2: 188, 193), ZMB 1964 (1: 134). Chile, Algarobbo: GCRL 12618 (1: 122), GCRL 12619 (5: 154-167), USNM uncat. (2: 122, 158). Chile, Arica: MNHNC uncat. (out of P.6.414; 1). Chile, Valparaiso and vicinity: MNHNC P.6.067 (2: 118, 156), MNHNC P.6.405 (3: 47-167), MNHNC P.6.407 (4), USNM $258788(14: 38-181)$, USNM UNCAT. ( $1: 35)$, USNM UNCAT. (3: 100-111). Peru: BMNH 1868.1.11.22-3 (2: 169, 179), CAS-SU 48836 ( 1 : 126). 
Exallias JORDAN AND EVERMANN

Exallias Jordan and Evermann, 1905:503 (type species Salarias brevis Kner, 1868a and b, by original designation).

Gloriella Schultz, 1941:17 (type species Cirripectes caninus Herre, 1936 = Salarias brevis Kner, $1868 \mathrm{a}$ and $\mathrm{b}$, by original designation). Leoblennius Reid, 1943:382 (type species Leoblennius schultzi Reid, 1943 = Salarias brevis Kner, $1868 \mathrm{a}$ and $\mathrm{t}$, by original designation).

Diagnosis. Distinguished from all other salariin blennies by a unique transverse row of nuchal cirri, in which the lateral cirri progressively increase in length toward the nape; a single fleshy flap associated with each of the two anteriormost sensory pores in each dentary series; and a relatively long and deep-bodied ophioblennius larval stage.

Nomenclatural discussion. Jordan and Evermann (1905) felt that Salarias brevis was sufficiently distinct from other blennies to warrant generic recognition and described the genus Exallias as a monotypic genus for the reception of $\underline{\mathrm{S}}$. brevis. Fowler (1928) did not believe that the characters used by Jordan and Evermann (1905) warranted generic distinction of the form and made Exallias a subgenus of cirripectes. The name Exallias was not used again until strasburg and Schultz (1953) reviewed the genus and provided characters to distinguish it from Cirripectes. 
Salarias brevis Kner, 1868a:29, and 1868b:334 (Samoa).

Salarias leopardus Day, 1870:518 (Galle Harbor).

Cirripectes caninus Herre, 1936:284 (Ternate, Moluccas).

Ophioblennius phalacrus Clark, 1938:184 (Taiohae Bay, Nukuhiva I., Marquesas).

Leoblennius schultzi Reid, 1943:382 (Honolulu, Hawaii).

Description. Dorsal fin XII,12-13 (13 in $92.5 \%$ of specimens); anal fin II, 13-14 (14 in $90.0 \%$ ); total procurrent caudal-fin rays $11-14$; pelvic fin $I, 4$; vertebrae $10+19-21(20$ in $94.7 \%)=29-31$ (30 in 94. $7 \%$ ); last pleural ribs on vertebral centrum 12; last epipleural rib on vertebral centrum 18-21 (19-21 in 97.4\%); anal pterygiophores 1-1-1 or $1-1-2(1-1-1$ in $86.8 \%)$; nuchal cirri $28-39(30-37$ in $87.5 \%)$; supraorbital cirri 11-34 (highly variable with no strong mode); nasal cirri $8-26(10-17$ in $82.1 \%)$; Li tubes $0-6$; last LL tube on caudal-fin base (rarely, in advance of vertical from last dorsal-fin ray); no scalelike flaps along LL; lower lip heavily folded; gill rakers 17-22 (based on 37 specimens); pseudobranchial filaments on one side 9-15 (based on 37 specimens); dentary teeth 40-71 (based on 40 specimens); upper lip crenulae approximately 17-26; nuchal cirri in a continuous 
row across nape, rarely with a slight notch at the dorsal midline, with the length of individual cirri gradually increasing in length toward the middle of nape (lateral cirri as little as one-fourth length of those at nape); dorsal-fin membrane deeply incised above last dorsal-fin spine; dorsal-fin membrane attached to caudal fin in adults; cephalic pore system complex ( 3 or more pores at most positions; number of pores increases with increasing SL); mid-snout pores present (Figure 17); extra interorbital pore position present (Figure 17); 2 pore positions behind nuchal flap (Figure 17); male genital papilla with urogenital orifice located basally behind a single slender filament (less than $1.0 \mathrm{~mm}$ long) on a fleshy swelling behind anus (type IV; Figure 13); testes elongate, length equals about twice width; maximum SL about $100 \mathrm{~mm}$.

The smallest mature female (ova about $0.5 \mathrm{~mm}$ diameter) examined was about $65 \mathrm{~mm}$ SL. Males mature by about 60 m. SL.

Color in alcohol. Ophioblennius stage larvae have uniformly pale-brown body with several brown blotches on nape, cheeks, pectoral-fin base, and dorsal part of body beneath spinous-dorsal fin; dorsal fin transluscent with $5-6$ brown blotches on basal quarter of spinous-dorsal fin; pale-brown blotches basally on segmented-dorsal fin, blotches becoming faizter posteriorly; pectoral fin with several brown blotches on basal half of fin and dark-brown marginal band restricted to interradial membranes; otherwise, fins transluscent.

Juveniles have scattered brown spots, about half pupil diameter in size, on pale background on head, body, and fins; about 5-6 bars on body, each bar overlain with paired columns of spots. 
As specimens increase in size, spots increase in number and decrease in size. Adults (Figure 69) of both sexes with fairly dense covering of spots arranged in slusters of 5-10 roughly hexagonal-shaped spots separated from other clusters by narrow pale interspaces; overall pattern on body consisting of about 5-6 broad bars (comprising clusters of brown spots described above) with narrow pale interspaces; fleshy rugosities on anal-fin spines of males black; iris pale with 4-5 black spots equally spaced along outer margin; cirri mottled with brown and white.

Color in life. Herre (1939) described the life colors of an ophioblennius stage larva as "yellow, with large brown spots between pectoral and dorsal, and small brown spots all over head and on pectoral base; 10 blackish-brown spots or vertical bands on dorsal; brown spots on pectoral, tips of rays black; other fins colorless." Juveniles (Masuda et al., 1975) have a white head and body with yellowish-brown spots. Spots on head behind eye are dark brown and typically darker than other spots. All fins are yellow with small brown spots.

Adult female coloration (Masuda et al., 1975; Randall, 1982b) is similar to that of juveniles except the spots are brown, smaller, and more densely spaced. Adult males (Masuda et al., 1984; Randal1, 1981) are essentially bicolored, with about anterior third of body similar in coloration to that of juveniles and females, and posterior portion of body, including dorsal, caudal, and basal part of anal fins, almost uniformly scarlet (most of the red color is concentrated in the spots). Most spots have a small black center. Light parts of iris vary in color from white to yellow. 


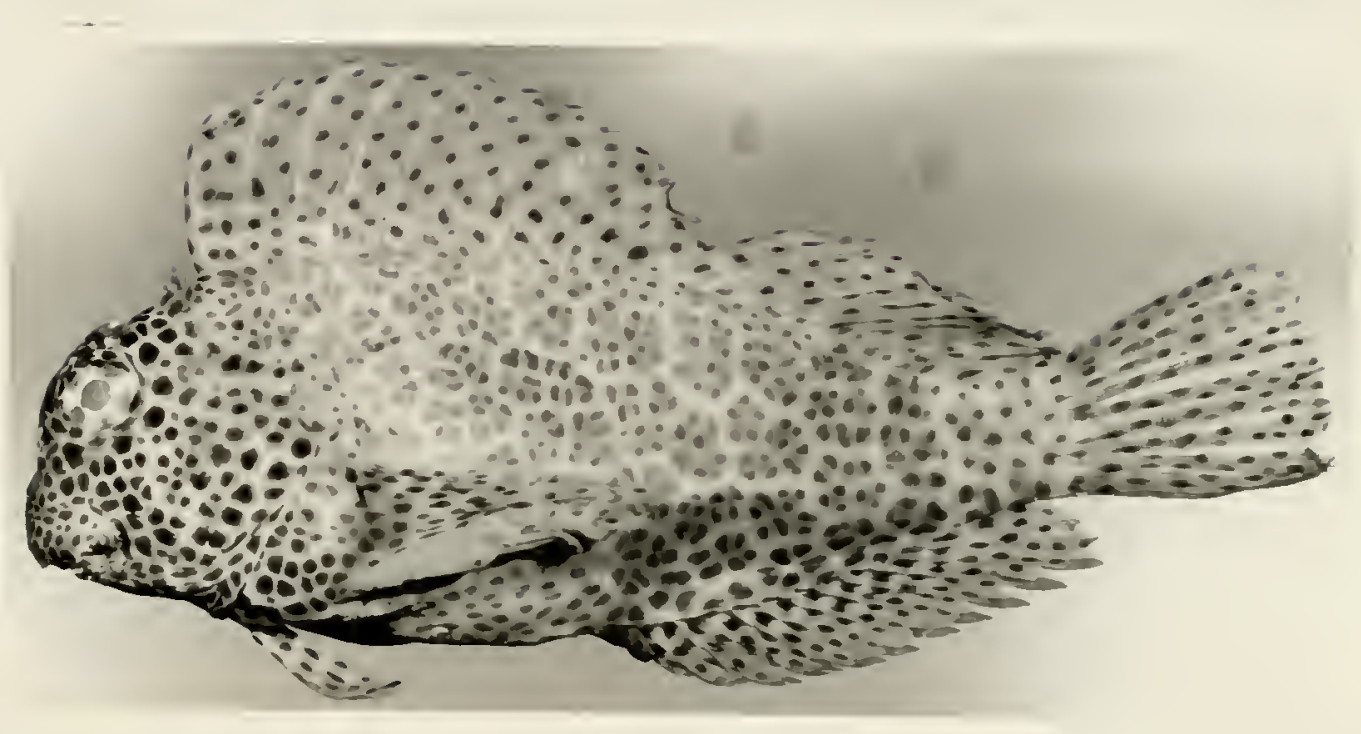

Figure 69. Male Exallias brevis, Chagos Archipelago (ROM uncat.: $71 \mathrm{~mm}$ SL). 
Distribution. Exallias brevis is a widely distributed coral reef inhabitant found throughout the tropical and subtropical Indo-Pacific. It occurs from South Africa to the northern Red Sea eastward to the Marquesas Islands (Figure 70).

Comments. Despite the extensive geographic range of Exallias brevis, most characters examined show very little variation among populations. It is possible that some geographic variation exists, but there are too few specimens from the same localities in museum collections on which to base any sound conclusions.

Nomenclatural discussion. Kner (1868a, b) described Salarias brevis based on a single specimen taken at Savaii, Samoa. Day (1870) described $\underline{\text { S. }}$ leopardus based on a single specimen "dredged up off Galle Harbor," Ceylon (=Sri Lanka). Day's description of $\underline{\text { S. }}$ leopardus clearly fits the species described by kner two years earlier and, thus, is a junior synonym of $\underline{s}$. brevis.

Schultz (1941) recognized both $\underline{\mathrm{C}}$. leopardus and $\underline{\mathrm{C}}$. brevis as valid species. The specimens Schultz (1941) referred to as $\underline{C}$. brevis were actually specimens of another species that was later described (Strasburg and Schultz, 1953) as C. Euscoguttatus. Chapman (1951) followed Schultz (1941) in recognizing $\underline{C}$. leopardus as a valid species. Strasburg and Schultz (1953) and Strasburg (1956) recognized Exallias as a distinct genus containing only $\mathrm{S}$. brevis, and listed Salarias lecpardus as a junior synonym.

Three other species (Cirripectes caninus Herre, Ophioblennius phalacrus Clark, and Leoblennius schultzi Reid) were each based on ophioblennius larval stage individuals. Each of these species are referable to Exallias brevis, and are junior synonyms of that species. 


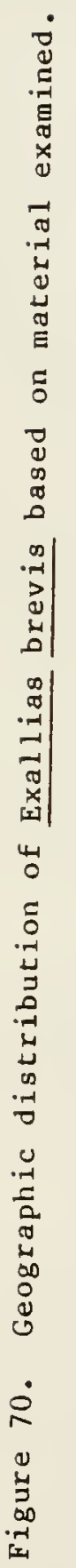




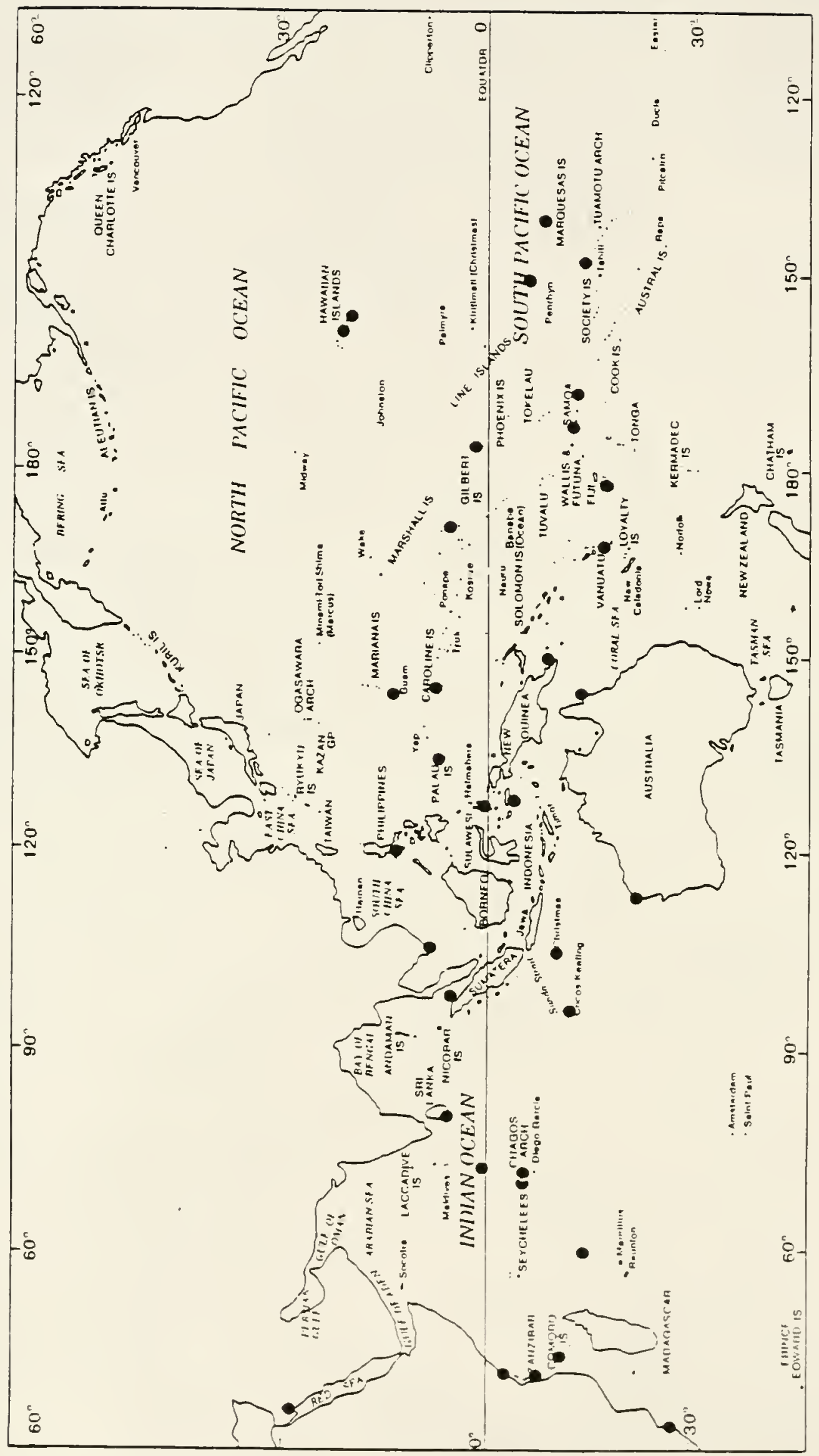


Material examined. Types: NMW 75387 (female: 77 sL), holotype of Salarias brevis, Savay, Samoa Islands, collected in 1868; CAS-SU 29082 (1: 23), holotype of Cirripectes caninus, Molluccas Islands, Ternate Island, collection date unknown; CAS 5566 (1: 25), holotype of Ophioblennius phalacrus, Marquesas Islands, Nuku Hiva, Taiohae Bay, 2-15 Oct. 1934; USNM 118037 (1: 25), holotype of Leoblennius schultzi, Hawaiian Islands, off Honolulu, Diamond Head Light, 6 May 1902.

Other material examined. South Africa: RUSI 67-11 (1: 53), RUSI 7514 (1: 42), RUSI 9628 (1: 53). Jumoros Islands: USNM 201875 (1). Tanzania: USNM 201873 (2). Kenya. USNM UNCAT. (1). Egypt: HUJF 7719 (2: 58, 59). Maldive Islands: SMF 13587 (1: 88). Cargados Carajos Shoals: USMM UNCAT. (1). Chagos Archipelago: ROM 50266 (1: 71), ROM 50267 (6: 56-77). Galle, Sri Lanka: USNM UNCAT. (1). Christmas Island, Indian Ocean: AMS I.20446-012 (1: 74), WAM P.26083-014 (1: 76). Malacca Strait: USNM 199420 (1). Indonesia: AMS I.19875-013 (1: 68). Philippines: USNM 122280 (1). Papua-New Guinea: USMM UNCAT. (1). Australia, Western Australia: WAM P.25368-010 (1: 58), WAM P.25827-001 (1: 61). Australia, Queensland: AMS I.19481-031 (2: 78, 85). Vanuatu (New Hebrides): AMS I.17473-040 (i: 74). Fiji Islands: ROM UNCAT. (2: 44, 83; WE 83-29), ROM UNCAT. (1: 61; WE 83-46), ROM UNCAT. (4: 41-91; WE 83-57). Caroline Islands: CAS 38831 (2 of 5 examined: 58, 87). Marshalls Islands: USNM 166786 (1). Hawaiian Islands: ZMB 6093 (1). Howland Island: USNM 198701 (1). Samoa: USNM 115703 (1). Vostok Island: USNM 200544 (1). Tuamotu Archipelago: BPBM UNCAT. (1). 
Springer (1982) cited the importance of cladistic analyses of organisms to what he termed vicariography (vicariance biogeography). Parenti (1981) referred to the combining of vicariance biogeography with hypotheses of phylogenetic systematics as cladistic biogeography. Rosen (1978), Platnick and Nelson (1978), and Nelson and Platnick (1981) discussed a method for transforming a cladogram of phylogenetic relationships into an area cladogram by replacing the terminal taxa with their respective geofiaphic distributions. If area cladograms for several different taxa are congruent, a general distributional pattern, a generalized track (Croizat et al., 1974), is supported. Once recognized, tracks can be used to search for geological or other vicariance events in earth history that explain the observed patterns. This was the approach taken by Springer (1982), but, as he pointed out, there are very few cladistic studies of Indo-Pacific organisms. The scarcity of cladistic studies also pertains to organisms in most other oceans.

In view of the scarcity of cladistic analyses of organisms with distributions similar to Cirripectes and its allies, I rely heavily on the relationships among taxa presented in Figures 1 and 15 and their corresnonding area cladograms (Figures 71 and 72 ). To facilitate comparison, the nodes of the area cladograms are numbered in the same 


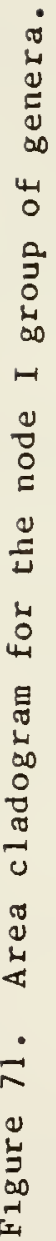




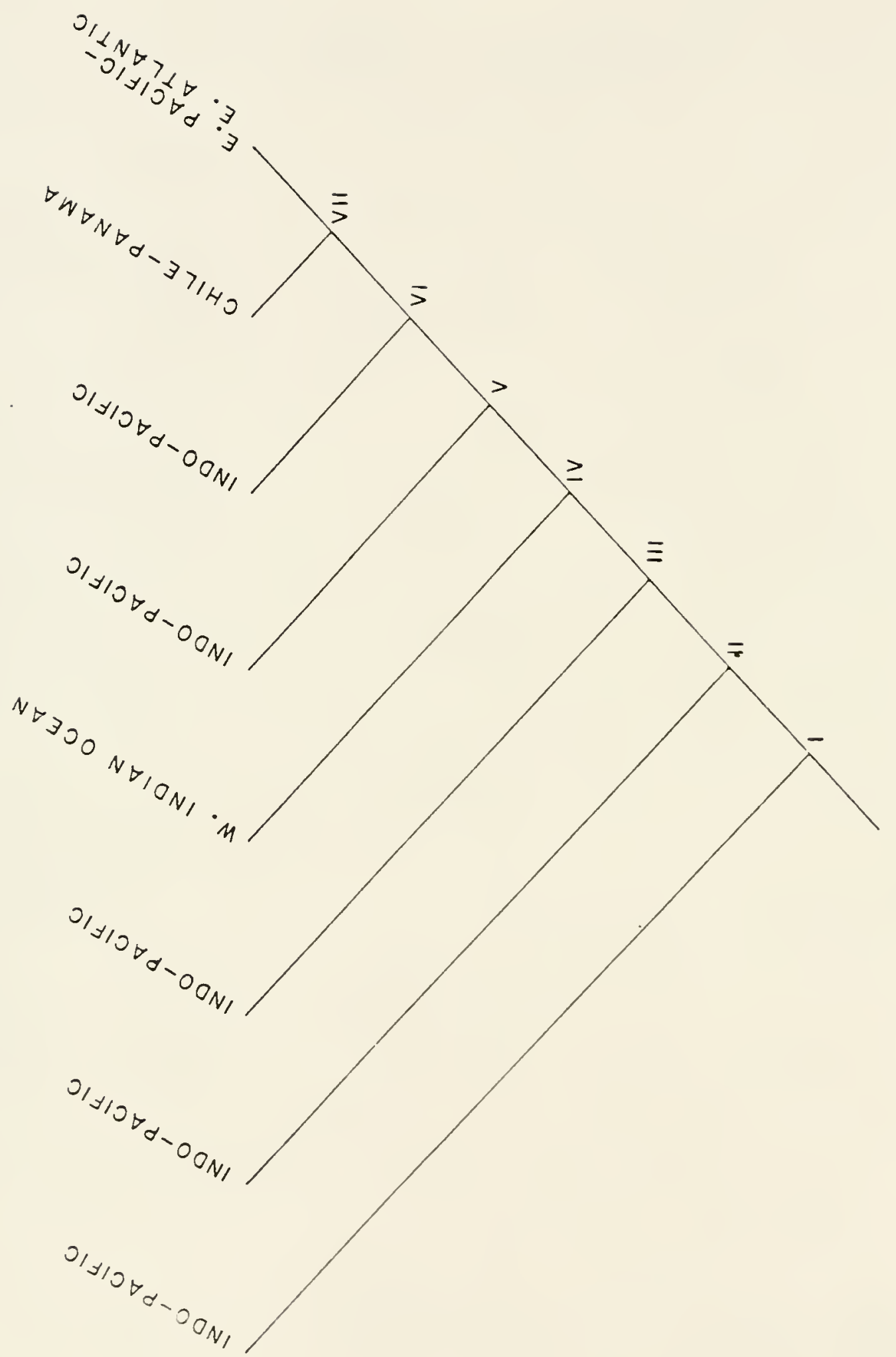




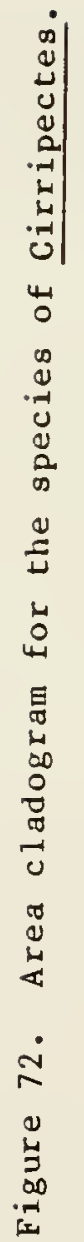




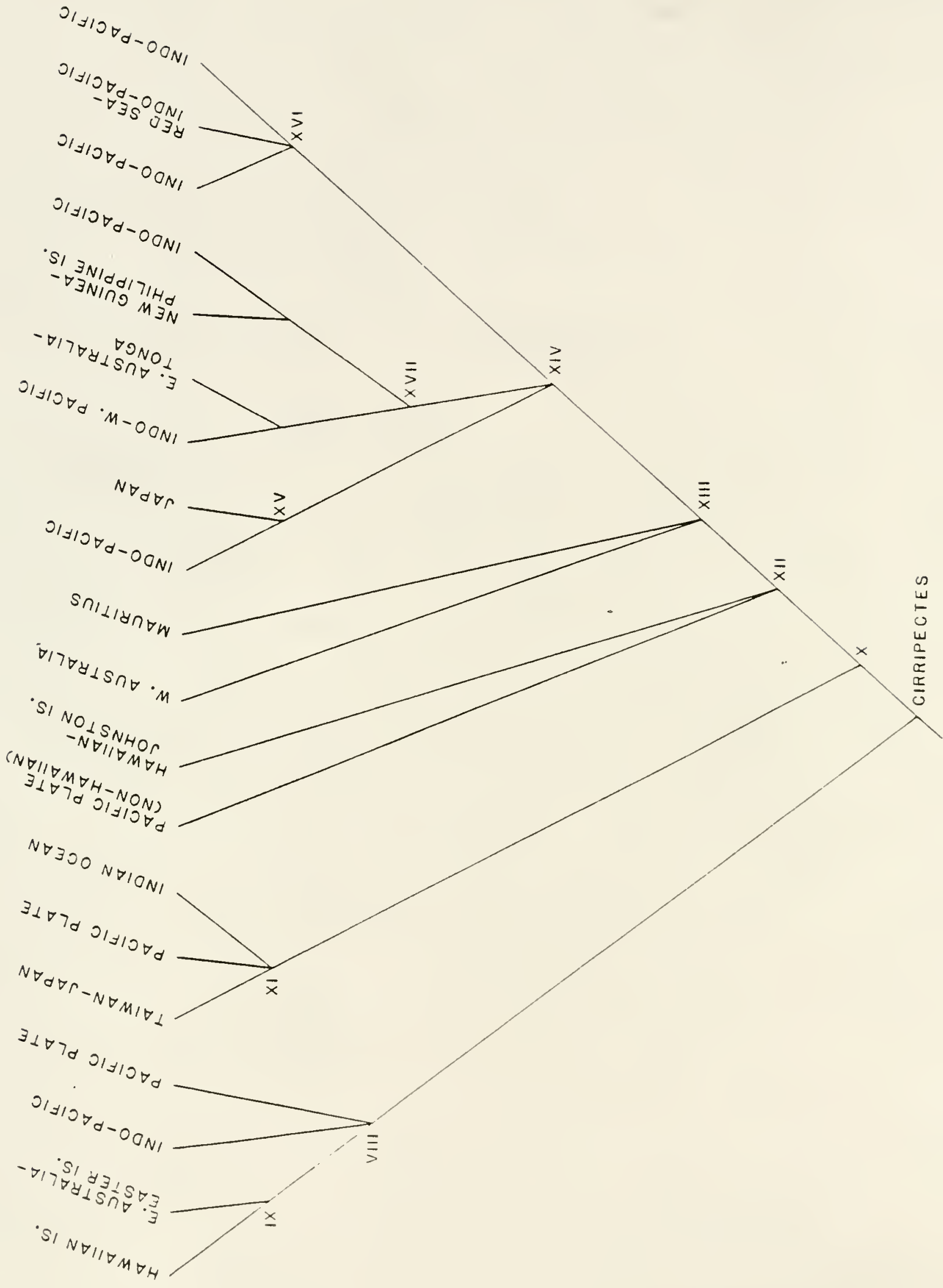


order as the nodes on the taxonomic cladograms. Although my analysis is restricted to blenniid taxa, it comprises several tracks that are congruent with tracks described by Rosen (1976), Springer (1982), and Vari (1978). I have not proposed vicariant events to explain the tracks. Cladistic analyses of other taxa are needed to support the tracks proposed herein.

I tentatively recognize the following eleven tracks: circumglobal tropical-to-temperate coastal marine--the entire family, (2) circumglobal tropical and subtropical--Salariini, (3) eastern Pacific to eastern Atlantic--Scartichthys and Ophioblennius (node VII group), (4) western South American-Caribbean Plate track-a subunit of number (3), (5) Indo-Pacific track--many salariin genera, (6) Pacific Plate track of Springer (1982), (7) Indo-West Pacific track of Vari (1978), (8) antitropical track-Cirripectes alboapicalis-C. obscurus group in the Pacific and the disjunct distribution of Pereulixia in the Indian Ocean, (9) eastern Indian Ocean-western Indian Ocean--C. $\underline{\text { randalli }}$ and $\underline{\text { C. hutchinsi, }}$ (10) track C of Vari (1973)--C. springeri, and (11) $\underline{\text { C. chelomatus }} \underline{\text { ch }}$ filamentosus track.

The present distribution of the Blenniidae describes a track comprising circumglobal tropical to temperate marine coastlines. The family track contains numerous less extensive tracks described by the geographical distribution of component taxa of the Blenniidae.

The track of the Salariini resembles that of the family: but does not inclure the Mediterranean Sea or temperate coastines (with a few 
exceptions). The majority of the salariin genera have an Indo-Pacific distribution, with only one (Entomacrodus) purportedly occurring from the Indo-Pacific to the Caribbean and Atlantic (Springer, 1967), but Entomacrodus may be polyphyletic (Springer, pers. comm.). Among the Salariini, only Scartichthys is restricted to the eastern Pacific and only Ophioblennius is distributed from the eastern Pacific to the islands of the eastern Atlantic Ocean (the eastern Pacific-eastern Atlantic track of Rosen, 1976). Each of these tracks (subunits of the Salariini track) is discussed below.

Rosen (1976) described the eastern Pacific-eastern Atlantic track as being the oldest transoceanic generalized track in this region of the world. Among the Salariini, the combined geographic distribution of Scartichthys and Ophioblennius is congruent with this track.

Scartichthys is distributed along the western coast of South America from central Chile to Panama, with $\underline{S}$. variolatus restriced to San Felix and San Ambrosio islands, and Juan Fernandez Islands, on the Nazca Plate. The other three species are restricted to the western margin of the South American Plate. Scartichthys gigas occurs from Panama to northern Chile. Scartichthys viridis occurs from southern Peru to the area around Valparaiso, Chile. These two species occur sympatrically in northern Chile and southern Deru. Scartichthys crapulatus, restricted to Chile, occurs sympatrically with $\underline{\text { S. viridis. }}$ Ophioblennius occurs sympatrically with Scartichthys from Peru to Panama, but Ophioblennius has a more extensive geographic range that extends northward to the Revillagigedos Islands and eastward to the eastern Atlantic Ocean. Ophioblennius comprises two recognized 
species, the eastern Pacific $\underline{0}$. steindachneri and the Caribbean-eastern Atlantic 0 . atlanticus, each with two subspecies (Springer, 1962; Springer, pers. comm., now believes all the subspecies should be accorded species-level recognition).

The geographic distributions of the node VII genera (Scartichthys and Ophioblennius) have geological correlations. The phylogenetic relationships between the node VII genera suggest a historic connection between western South America and the Caribbean. The split in the ancestral distribution may have occurred along the boundary between the South American and Caribbean plates. If this hypothesis is correct, the present-day sympatric occurrence of the node VII genera in northwestern South America is evidence of dispersal of the two sister genera.

The two species of Ophioblennius have an eastern Pacific-Caribbean relationship. This relationship presumably represents the division of a continuous ancestral population by the development of the Central American land barrier.

The Indo-Pacific track is a generalized track comprising two widely recognized tracks, the Pacific Plate track of Springer (1982) and the Indo-West Pacific track of Vari (1978). The Indo-?acific track is approximated by the distributions of Eirripectes quagga, ‥ auritus, ‥ perustus, $\underline{C}$. polyzona, and $\underline{C}$. stigmaticus. The combined distributions of the taxa at almost every node on the generic area cladogram, and many nodes on the Cirripectes area cladogram, approximate this generalized track. 
Springer (1982) demonstrated the presence of a generalized Pacific Plate track and also discussed numerous distribution patterns that fit the Indo-West Pacific track, the boundary between the two tracks being the western margin of the Pacific lithospheric plate. Among the species of Cirripectes, $\underline{C}$. Euscoguttatus is a Pacific Plate endemic associated in a trichotomy (node XI group) with the Japan-Taiwan endemic, $\underline{C}$. imitator, and the Indian Ocean endemic, $\underline{\text {. }} \underline{\text { gilberti. }}$ Another trichotomous sister-group relationship (node XII group) exists between the Pacific Plate endemic, $\underline{C}$. variolosus, the Hawaiian Islands-Johnston Island endemic, C. vanderiilti, and the primarily Indo-West Pacific node XIII group. The geographic distribution of $\underline{C}$. auritus, $\underline{\text { C. perustus, }}$ and the node XVI group is evidence of dispersal onto the Pacific Plate, probably via the Caroline Islands conduit described by Springer (1982).

The distributional track of the node VIII group comprises three subunits, antitropical Pacific, Pacific Plate, and Indo-Pacific components. The node IX sister group relationship between Cirripectes obscurus, the Hawaiian Islands endemic, and $\underline{C}$. alboapicalis, which is distributed south of $20^{\circ}$ S latitude from eastern Australia to Isla de Pascua (Easter Island), makes up the antitropical component. This antitropical component generally fits the pattern discussed by Randall (1982a) for Pacific taxa.

The Pacific Plate erdemic, $\underline{C}$. jenningsi, occurs in the intervening area between the antitropically distributed species, where it is distributed from the Gilbert Islands to the Tuamotu Archipelago. 
The geographic distribution of the last member of the node VIII

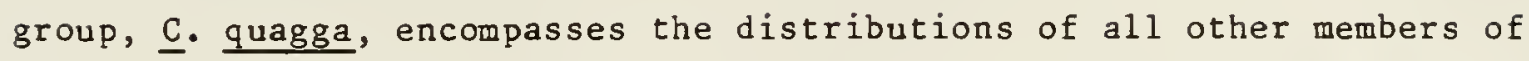
the node VIII group and extends into the western Indian Ocean. The geographic distribution of $\underline{C}$. quagga is evidence of dispersal, but its sister group within the node VIII group has not been determined; thus, the extent of its dispersal is as yet unknown.

Another subunit of the Indo-West Pacific track is an eastern Indian Ocean-western Indian Ocean track. This track is weakly supported by the node IV group and a trichotomy at node XIII. At node IV, Pereulixia kosiensis is restricted to the western Indian Ocean, where it appears to have an antitropical distribution. It occurs in South Africa, Mozambique, and in Pakistan.(Hoda, 1980). As there has been relatively little collecting effort expended along the eastern African coast, the known distribution of Pereulixia may be a collecting artifact. Other members of the node IV group are either widespread Indo-Pacific taxa that are at least partially sympatric with Pereulixia, or are restricted to the eastern Pacific and/or Caribbean regions. Although node XIII is an unresolved trichotomy, two of the taxa, the Cargados Carajos Shoals-Mauritius endemic, Cirripectes

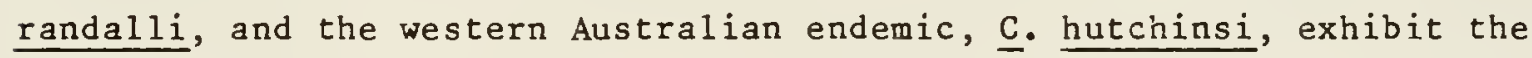
eastern Indian Ocean-western Indian Ocean distribution pattern. In addition, the occurrence of these two species south of $20^{\circ} \mathrm{S}$ latitude (although $\underline{\mathrm{C}}$. randalli occurs at latitudes slightly lower than $20^{\circ} \mathrm{S}$ ) concurs with the southern end of the antitropical distribution track. Within the node XVII group there are two tracks. The first track is represented by the distribution of Cirripectes springeri, which 
occurs from the Philippines and along the northern coast of New Guinea to the Solomon Islands. This distributional pattern fits within track C of Vari (1978). Geologically, the pattern follows the western margins of the Philippine and Pacific Plates (essentially follows the Melanisian borderlands, a geologically recognized tectonic unit, Springer, pers. comm.). The sister group of $\underline{C}$. springeri is uncertain, as the relationships within the node XVII group are unresolved. If $\mathrm{C}$. perustus is its sister species, then there is evidence of dispersal in that their ranges overlap in the Philippines and the Bismarck Archipelago. The distribution of $\underline{C}$. perustus is completely within that of the more widely distributed $\underline{C}$. filamentosus, but the distribution of

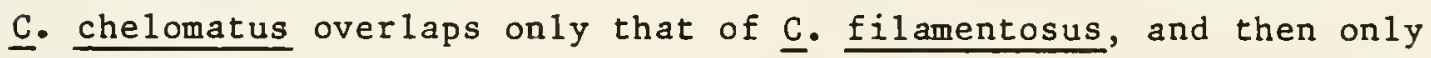
slightly.

The combined distribution of $\underline{\mathrm{C}}$. chelomatus and $\underline{\mathrm{C}}$. filamentosus describes a second track consisting of a Coral Sea to Tonga Islands component (Cirripectes chelomatus) and an Indo-West Pacific component (C. filamentosus). Evidence of dispersal within this group is the sympatry of the two species in the northern Coral Sea.

The validity of some of the tracks discussed is relatively certain (i.e. Pacific Plate track, Indo-Pacific track, eastern Pacific-eastern Atlantic track), but most of the other tracks I discussed have not been corroborated. The area cladograms (Figures 71 and 72 ) predict that these tracks will be congruent with area cladograms for other taxa in these geographic areas, but this prediction can only be tested by phylogenetic analyses of additional taxa. I hope my hypotheses will help to stimulate such studies. 
APPENDIX 
Table 5. Table of material used for osteological studies.

$\underline{\text { Family }}$

Dactyloscopidae

Tripterygiidae

Chaenopsidae

Clinidae

Labrisomidae
Dactylagnus mundus

USNM 270277 ( 1 )

USNM $270365 （ 2)$

USNM 200385

Dactyloscopus crossotus USNM 270360

Dactyloscopus tridigitatus

USNM $269550 \quad(2)$

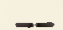

Enneanectes altivelis

UF 16179 (2)

-- Hemiemblemaria simulus

UF 19112 (1)

Acanthemblemaria aspera

UF 11881 (2)

Emblemariopsis signifera UF uncat. (1)

-- Gibbonsia elegans erroli USNM 200386

Paraclinus nigripinnis

UF uncat. (1)

Labrisomus nuchipinnis UF uncat. (1)

Blenniidae

$\begin{array}{ll}\text { Blennitini } & \text { Blennius normani } \\ \text { Blennius ocellaris }\end{array}$

USNM $199533(2)$

USNM 48387 ( 1 )

USNM 205157（1）

Salariini

Alticus saliens

USNM 227848

Alloblennius pictus 
Table 5. Continued.

Family

Tribe

Snecies

Catalog Numbers

Andamia reyi

US NM $227797 \quad(2)$

Antennablennius bifilum USNM 200207 (2)

Atrosalarias fuscus USNM 227555 (2)

Cirripectes castaneus USNM 228189 (2)

Cirripectes chelomatus USNM 258751 (2)

Cirripectes filamentosus USNM 228108 (2)

Cirripectes fuscoguttatus USNM 198700 (1)

USNM 200612 (1)

USNM 227867 ( 1 )

Cirripectes gilberti

ROM 46597 (1)

Cirripectes imitator

USNM $227980 \quad(1)$

Cirripectes jenningsi USNM 200615 (5)

Cirripectes obscurus BPBM uncat. (2)

Cirripectes perustus ROM 46594 (1)

Cirripectes quagga $\quad$ USNM 111603 (4)

USNM $198751 \quad(2)$

USNM $200605 \quad(2)$

Cirripectes polyzona USNM 227688 (2)

Cirripectes randalli BPBM 20165 (1)

Cirripectes springeri USNM 228287 (1)

US NM 228294 (1)

Cirripectes stigmaticus USNM 223457 (2) 
Table 5. Continued.

$\underline{\text { Family }}$

$\underline{\text { Tribe }}$

$\underline{\text { Species }}$

Catalog Number

Cirripectes vanderbilti USNM 142114（2)

Cirripectes variolosus USNM 200614 (3)

Crossosalarias macrospilus USMM 205711 (1)

Ecsenius bicolor USNM 211914 (2)

Ecsenius yaeyamaensis USNM 211924 (2)

Entomacrodus cymatobiotus USNM 198654 (2)

Entomacrodus epalzeochilus USNM 269464 (1)

Entomacrodus nigricans UF 17048 (2)

Entomacrodus stellifer USNM 132795 (1)

Exallias brevis USNM 224383 (2)

USNM 200544（1）

Hirculops cornifer $\quad$ USNM 200030 (2)

Istiblennius gibbifrons USM 228313 (2)

Istiblennius lineatus UF uncat. (1)

Litobranchus fowleri USM 227798 (1)

Mimcblennius atrocinctus USNM 197980 (1)

USNM $216676 \quad(2)$

Nannosalarias nativitatus USNM 201369 (1)

Ophioblennius at lanticus macclurei UF $10746 \quad(2)$

Pereulixia kosiensis USNM 197637 (1)

Praealticus sp. USNM 228318 (2) 
Table 5. Continued.

Family

Trjbe

Species

Rhabdoblennius snowi

Scartichthys gigas

Stanulus seychellensis
Meiacanthus grammistes

Enchelyurus kraussi

Omobranchus punctatus

Chasmodes bosquianus

bosquianus

Chasmodes bosquianus

longimaxilla

Chasmodes saburrae

Hypleurochilus geninatus

Hypsoblennius hentz

Parablennius marmoreus

Scartella cristata
Catalog Number

USNM 228315 (2)

USNM 200396（2）

USNM 227556 (2)

US NM $227981 \quad(2)$

USNM 228316 (2)

USNM 201363 (1)

USNM 200609 (1)

USNM $174330 \quad(1)$

UF 28296 (1)

USA 6005 (3)

UF uncat. (1)

UF uncat. (2)

UF 19621 (1)

UF 2584 (1)

UF 11021 (2)

UF 11020（2） 


\section{LITERATURE CITED}

Allen, G. R., D. F. Hoese, J. R. Paxton, J. E. Randall, B. C. Russell, W. A. Starck II, F. H. Talbot, and G. P. Whitley. 1976. Annotated checklist of the fishes of Lord Howe Island. Records of the Australian Museum 30:365-454.

Alleyne, H. G., and W. Macleay. 1877. The ichthyology of the Chevert Expedition. Proceedings of the Linnean Society of New South Wales $1: 321-359$.

Bath, H. 1977. Revision der Blenniini. Senckenbergiana Biologica $57: 167-234$.

Bleeker, P. 1868. Description de deux especes rouvelles de blennioides de 1'Inde Archipelagique. Verslagen en Mededeelingen der Koninklikje Akademie van Wetenschappen, Letterkunde, en Schoone Kunsten te Amsterdam, ser. 2, 2:278-280.

Bleeker, P. 1983. Atlas Ichthyologique. Smithsonian Institution Press, Washington. Vol. 11:1-22, pls. 421-447.

Borodin, N. A. 1927. A new blenny from the Hawaiian Islands. American Museum Novitates 281:1-2.

Borodin, N. A. 1928. Exallias obscurus Borodin vs. Cirripectes alboapicalis (Ogilby). Copeia 1927:53-54.

Carlson, B. A. 1981. A new Indo-Pacific fish of the genus Cirripectes (Blenniidae, Salariini). Pacific Science 34:407-414.

Chapman, W. M. 1951. Family Blenniidae. Pages 242-355 in L. E. de Beaufort and W. M. Chapman. Fishes of the Indo-Australian Archipelago. E. J. Brill, Leiden, Holland. Vol. 9.

Clark, H. W. 1938. The Templeton Crocker Expedition of 1934-35. No. 36. Additional new fishes. Proceedings of the California Academy of Sciences, ser. 4, 22:179-185.

Cohen, D. M. 1956. Notes on the identity of several salariine blennies of the genus Scartichthys from western South America. Copeia 1956:246-248.

Croizat, L., G. J. Nelson, and D. E. Rosen. 1974. Centers of Origin and related concepts. Systematic Zoology 22:265-287.

Cuvier, G. F. L. C. D., and A. Valenciennes. 1836. Histoire naturelle des poissons. F. G. Levrault, Paris, France. Vol. 11:1-506. 
Day, F. 1870. Remarks on some fishes in the Calcutta Museum. Part 1. Proceedings of the Scientific Meetings of the Zoological Society of London for the year 1869:511-527.

Day, F. 1888. Supplement to the fishes of India. Williams and Norgate Publishers, London, England. Pages 779-816.

Day, F. 1889. The fauna of British India, including Ceylon and Burma. Fishes. Taylor and Francis, London, England. Vol. 2, 509 pp.

De Buen, F. 1963. Los peces de la Isla de Pascua. Boletin de Ia Sociedad de Biologia de Concepcion, Chile, 1960-61, 35-36:3-80.

Dingerkus, G., and L. D. Uhler. 1977. Enzyme clearing of alcian blue stained whole small vertebrates for demonstration of cartilage. Stain Technology 52:229-232.

Fowler, H. W. 1923. New or little-knowr. Hawaiian fishes. Occasional Papers of the Bernice P. Bishop Museum 8:373-392.

Fowler, H. W. 1927. Notes on Exallias obscurus Borodin. Copeia $1927: 91-92$.

Fowler, H. W. 1928. The fishes of Oceania. Memoir of the Bernice P. Bishop Museum 10:1-540.

Fowler, H. W. 1938. The fishes of the George Vanderbilt South Pacific Expedition. Academy of Natural Sciences of Philadelphia, Monograph $2: 1-349$.

Fowler, H. W. 1940. Fishes obtained in Chile by Mr. D. S. Bullock. Proceedings of the Academy of Natural Sciences of Philadelphia $92: 171-190$.

Fowler, H. W. 1946. A collection of fishes obtained in the Riu Kiu Islands by Captain Ernest R. Tinkham A. U. S. Proceedings of the Academy of Natural Sciences of Philadelphia 98:123-218.

Fowler, H. W., and S. C. Ball. 1924. Descriptions of new fishes obtained by the Tanager Expedition of 1923 in the Pacific Islands west of Hawaii. Proceedings of the Academy of Natural Sciences of Philadelphia 76:269-274.

Fukao, R. 1984. Review of the Japanese fishes of the genus Cirripectes (Blenniidae) with description of a new species. Japanese Journal of Ichthyology $31: 105-121$.

Garman, S. 1903. Some fishes from Australasia. Bulletin of the Museum of Comparative Zoology, Harvard 39:229-241. 
George, A., and V. G. Springer. 1981. Revision of the clinid fish tribe Ophiclinini, including five new species, and definition of the family Clinidae. Smithsonian Contributions to Zoology $307: 1-31$.

Gosline, W. A. 1955. The inshore fish fauna of Johnston Island, a Central Pacific Atoll. Pacific Science 9:442-480.

Gunther, A. C. L. G. 1861. Catalogue of the Acanthopterygian fishes in the collection of the British Museum. N.P., London, England. Vol. 3, $586 \mathrm{pP}$.

Gunther, A. C. L. G. 1877. Andrew Garrett's Fische der Sudsee. VI. Journal des Museum Godeffroy 13:169-216, p1s. 101-120.

Hennig, W. 1966. Phylogenetic systematics. University of Illinois Press, Urbana, Illinois. 263 pp.

Herre, A. W. C. T. 1936. Fishes in the Zoological Museum of Stanford University, III. New genera and species of gobies and blennies and a new Myxus, from the Pelew Islands and Celebes. Philippine Journal of Science 59:275-287.

Herre, A. W. C. T. 1939. The Philippine blennies. Philippine Journal of Science $70: 315-373$.

Herre, A. W. C. T. 1953. Check 1 ist of Philippine fishes. Department of the Interior, Research Report 20:1-977.

Hildebrand, S. F. 1946. A descriptive catalog of the shore fishes of Peru. United States National Museum Bulletin 189:1-530.

Hoda, S. M. S. 1980. Blenniid fishes from the Karachi Coast. Proceedings of the lst Pakistani Congress of Zoology B:437-447.

Kner, R. 1868a. Uber neue Eische aus dem Museum der Herren Johann Casar Godeffroy \& Sohn in Hamburg. Sitzungsberichte der Kaiserlichen Akademie der Wissenschaften in Wien 58, part 1, 26-31.

Krer, R. 1868b. Uber neue fische aus dem Museum der Herren Johann Casar Godeffroy \& Sohn in Hamburg. Sitzungsberichte der Kaiserlichen Akademie der Wissenschaften in Wien 58(part 7):334.

Kner, R., and F. Steindachner. 1866. Neue fische aus der Herren Joh. C. Godeffroy \& Sohn in Hamburg. Sitzungsberichte der Kaiserlichen Akademie der Wissenschaften in Wien 54:356-395.

Jones, S., and M. Kumaran. 1980. Fishes of the Laccadive Archipelago. Nature Conservation and Aquatic Sciences Service, Trivandrum, India. $760 \mathrm{pp}$. 
Jordan, D. S., and B. W. Evermann. 1896. A check-list of the fishes and fish-like vertebrates of North and Middle America. United States Commission of Fish and Fisheries, Report of the Commissioner for the Year Ending June 30. 1895(21):209-584.

Jordan, D. S., and B. W. Evermann. 1898. The fishes of North and Middle America. United States National Museum Bulletin 47(Part 3) : 2183-3136.

Jordan, D. S., and B. W. Evermann. 1905. The aquatic resources of the Hawaiian Islands. Part I. The shore fishes. United States Bureau of Fisheries, Bulletin (1903) 23:1-574.

Leis, J. M., and D. M. Rennis. 1983. The larvae of Indo-Pacific coral reef fishes. University of Hawaii Press, Honolulu, Hawaii. 269 pp., 75 pls.

Leviton, A. E., R. H. Gibbs, Jr., E. Heal, and C. E. Dawson. 1985. Standards in herpetology and ichthyology: Part I. Standard symbolic codes for institutional resource collections in herpetology and ichthyology. Copeia 1985:802-832.

Lotan, R. 1970. Systematic remarks on fishes of the family Salariidae in the Red Sea. Israel Journal of Zoology 18:363-378.

Maddison, W. P., M. J. Donoghue, and D. R. Maddison. 1984. Outgroup analysis and parsimony. Systematic Zoology 33:83-103.

Masuda, H., K. Amaoka, C. Araga, T. Uyeno, and T. Yoshino, editors. 1984. The fishes of the Japanese Archipelago. Tokai University Press, Tokyo, Japan. 437 pp., 370 pls.

Masuda, H., C. Araga, and T. Yoshino. 1975. Coastal fishes of southern Japan. Tokai University Press, Tokyo, Japan. 382 pp.

Masuda, H., C. Araga, and T. Yoshino. 1980. Coastal fishes of southern Japan. Revised edition, Tokai University Press, Tokyo, Japan. 382 pp.

McCulloch, A. P., and F. A. McNeill. 1918. Some Australian blennioid fishes. Australian Museum Records 12:1-25, 4 pls.

Nelson, G., and N. Platnick. 1981. Systematics and Biogeography Cladistics and Vicariance. Columbia University Press, New York, New York. 567 pp.

Norman, J. R. 1943. Notes on the blennioid fishes. I. A provisional synopsis of the genera of the family Blenniidae. Annals \& Magazine of Natural History, Ser. 11, 10:793-812.

Ogilby, J. D. 1889. The reptiles and fishes of Lord Howe Island. Memoirs of the Australian Museum 2:51-74. 
Ogilby, J. D. 1899. Additions to the fauna of Lord Howe Island. Proceedings of the Linnean Society of New South Wales 23:730-745.

Parenti, L. R. 1981. A phylogenetic and biogeographic analysis of cyprinodontiform fishes (Teleostei, Atherinomorpha). Bulletin of the American Museum of Natural History 168:335-557.

Philippi, R. A. 1896. Peces nuevos de Chile. Anales de Ia Universidad, Chile 93:375-390.

Platnick, N., and G. J. Nelson. 1978. A method of analysis for historical biogeography. Systematic Zoology 27:1-16.

Randall, J. E. 1955. Fishes of the Gilbert Islands. Atoll Research Bulletin $47: 1-243$.

Randal1, J. E. 1981. Underwater guide to Hawaiian Reef fishes. Harrowood Books, Newtown Square, Pennsylvannia. 71 pp.

Randal1, J. E. 1982a. Examples of antitropical and antiequatorial distribution of Indo-West-Pacific fishes. Pacific Science $35: 197-209$.

Randal1, J. E. 1982b. The divers guide to Red Sea Reef Fishes. Immel Publishing, London, England. 2 pp. + 325 figs.

Rehder, H. A. 1981. The marine molluscs of Easter Island (Isla de Pascua) and Sala y Gomez. Smithsonian Contributions to Zoology 289:1-167.

Reid, E. D. 1943. Review of the genera of blennioid fishes related to Ophioblennius. Journal of the Washington Academy of Sciences 33:373-384.

Rosen, D. E. 1976. A vicariance model of Caribbean biogeography. Systematic Zoology 24:431-464.

Rosen, D. E. 1978. Vicariant patterns and historical explanation of biogeography. Systematic Zoology 27:159-188.

Russell, B. C. 1983. Annotated checklist of the coral reef fishes in the Canricorn-Bunker Group, Great Barrier Reef, Australia. Great Barrier Reef Marine Park Authority, Australia. 184 pp.

Schultz, L. P. 1941. The species of Cirripectes Swainson and a new genus of blennioid fishes from the tropical Pacific. Copeia $1941: 17-20$.

Schultz, L. P. 1943. Fishes of the Phoenix and Samoan Islands collected in 1939 during the Expedition of the U. S. S. "Bushnel1." United States National Museum Bulletin 180:1-316. 
Schultz, L. P., and W. M. Chapman. 1960. Fishes of the Marshall and Marianas Islands. Subfamily Salariinae. United States National Museum Bulletin 202:30乞-j72.

Seale, A. 1901. Report of a mission to Guam. Part 2. Fishes. Occasional Papers of the Bernice P. Bishop Muswum 1:61-128.

Shen, S.-C. 1984. Coastal fishes of Taiwan. Shih-chieh Shen, Taipei, Taiwan. $190 \mathrm{pp}$.

Smith, H. M. 1934. The blenniid fishes of Siam, with descriptions of new species. Journal of the Siam Society, Natural Histroy Supplement 9:315-323.

Smith, J. L. B. 1947. New Species and new records of fishes from South Africa. Annals \& Magazine of Natural History, Series 11, $13: 793-821$.

Smith, J. L. B. 1959. Fishes of the families Blenniidae and Salariidae of the western Indian Ocean. Rhodes University, Department of Ichthyology, Ichthyological Bulletin 14:229-252.

Smith, J. L. B., and M. M. Smith. 1963. The fishes of Seychelles. Rhodes University, Grahamstown, South Africa. 215 pp.

Smith-Vaniz, W. F. 1976. The saber-toothed blennies, tribe Nemophini (Pisces: Blenniidae). Academy of Natural Sciences of Philadelphia, Monograph 19:1-196.

Smith-Vaniz, W. F., and V. G. Springer. 1971. Synopsis of the tribe Salarini, with description of five new genera and three new species (Pisces: Blenniidae). Smithsonian Contributions to Zoology $73: 1-72$.

Snyder, J. 0. 1908. Descriptions of eighteen new species and two new genera of fishes from Japan and the Riu Kiu Islands. Proceedings of the United States National Museum 35:93-111.

Springer, V. G. 1962. A review of the blenniid fishes of the genus Ophioblennius Gill. Copeia 1962:426-433.

Springer, V. G. 1967. Revision of the circumtropical shorefish genus Entomacrodus. Proceedings of the United States National Museum 122:1-150.

Springer, V. G. 1968a. The Indo-Pacific blenniid fish genus Stanulus, with description of a new species from the Great Barrier Reef (Blenniidae; Blenniinae; Salariini). Proceedings of the Biological Society of Washington $81: 111-121$.

Springer, V. G. 1968b. Osteology and classification of the fishes of the family Blenniidae. United States National Museum Bulletin $284: 1-85$. 
Springer, V. G. 1970. The blennies. Tropical Fish Hobbyist 19(176, no. 2): 54-66.

Springer, V. G. 1971. Revision of the fish genus Ecsenius (Blenniidae, Blenniinae, Salariini). Smithsonian Contributions to Zoology $72: 1-74$.

Springer, V. G. 1982. Pacific Plate biogeography, with special reference to shorefishes. Smithsonian Contributions to Zoology $367: 1-182$.

Springer, V. G., and W. F. Smith-Vaniz. 1972. A new tribe (Phenablennini) and genus (Phenablennius) of blenniid fishes based on Petroscirtes heyligeri Bleeker. Copeia 1972:64-71.

Steindachner, F. 1876. Ichthyologische Beitrage (V). Sitzungsberichte der Kaiserlichen Akademie der Wissenschaften in țien 74:49-240.

Strasburg, D. W. 1956. Notes on the blennioid fishes of Hawaii with descriptions of two new species. Pacific Science 10:241-267.

Strasburg, D. W., and L. P. Schultz. 1953. The blenniid fish genera Cirripectus and Exallias with description of two new species from the tropical Pacific. Journal of the Washington Academy of Sciences $43: 128-135$.

Swainson, W. 1839. The natural history and classification of fishes, amphibians, and reptiles, or monocardian animals. Longman, Orme, Brown, and Longmans, London, England. Vol. 2. 452 pp.

Vari, R. P. 1978. The terapon perches (Percoidei, Teraponidae): A cladistic analysis and taxonomic revision. Bulletin of the American Museum of Natural History 159:175-340.

Williams, J. T. 1985. Cirripectes imitator, a new species of western Pacific blenniid fish. Proceedings of the Biological Society of Washington 98:533-538.

Williams, J. T., and L. A. Mauge. 1983. Cirripectes chelomatus, a new species of salarine fish (Pisces, Blenniidae). Bulletin du Museum National d'Histoire Naturelle, Paris, serie 4, 5(section A) : 1139-1 149 . 


\section{BIOGRAPHICAL SKETCH}

Jeffrey Taylor Williams, the son of Jesse G. and Betty Williams, was born in Honolulu, Hawaii, in 1953. He graduated from Mountain Brook High School in Birmingham, Alabama, in 1971. After spending one year at the University of Mississippi, he went to Florida State University where he became interested in ichthyology. He graduated in 1975 and went on to the University of South Alabama to begin a graduate program in ichthyology. He was elected to Phi Kappa Phi and Sigma Xi and received his master's degree in 1979. He then began a doctoral program in the Department of Zoology at the University of Florida. After completing his coursework, but before finishing his dissertation, he left Florida in 1983 to take a job at the National Museum of Natural History, Smithsonian Institution in Washington, D.C. He continued to work on his dissertation and completed it in 1986. He is married to Karen Anderson Williams. 
I certify that I have read this study and that in my opinion it conforms to acceptable standards of scholarly presentation and is fully adequate, in scope and quality, as a dissertation for the degree of Doctor of Philosophy.

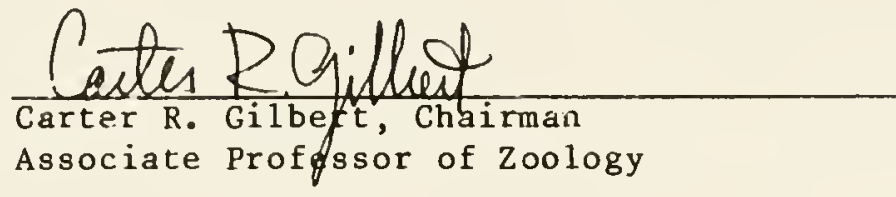

I certify that I have read this study and that in my opinion it conforms to acceptable standards of scholarly presentation and is fully adequate, in scope and quality, as a dissertation for the degree of Doctor of Philosophy.

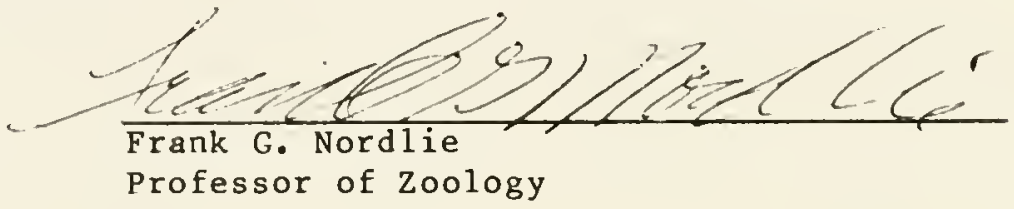

I certify that I have read this study and that in any opinion it conforma to acceptable standards of scholarly presentation and is fully adequate, in acope and quality, as a dissertation for the degree of Doctor of Philosophy.

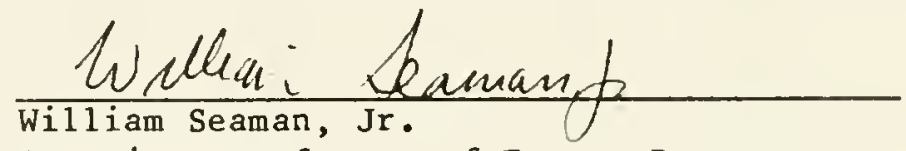
Associate Professor of Forest Resources and Conservation

I certify that I have read this study and that in my opinion it conforms to acceptable standards of scholarly presentation and is fully adequate, in acope and quality, as a dissertation for the degree of Doctor of Philosophy.

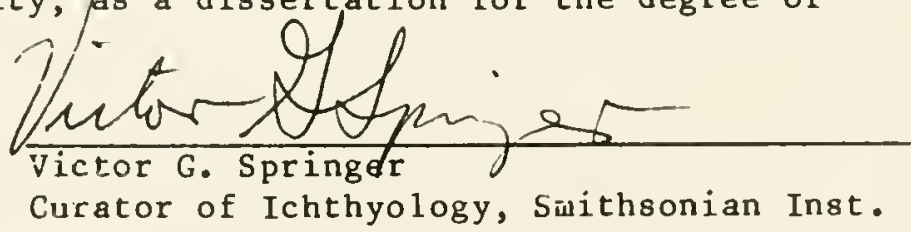

This disaertation was submitted to the Graduate Faculty of the Department of Zoology in the College of Liberal Arts and Sciences and to the Graduate School and was accepted as partial fuifillment of the requirements for the degree of Doctor of Philosophy.

May 1986

Dean, Graduate School 
UNIVERSITY OF FLORIDA

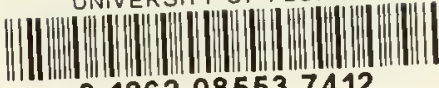

31262085537412 